\title{
30TH INTERNATIONAL SYMPOSIUM ON THE AUTONOMIC NERVOUS SYSTEM
}

Wyndham Grand Clearwater Beach, Florida

November 6-9, 2019

Preliminary Program

WEDNESDAY, NOVEMBER 6, 2019

7:00-8:30 AM

Registration

Dunes Foyer

Continental Breakfast

Pelican/Heron

8:30-8:45 AM

President's Welcome

Satish R. Raj, M.D.

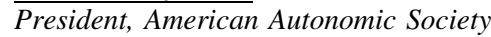

Dunes I-III

8:45-9:45 AM Plenary Lecture 1: Robertson Plenary Lecture

Impact of obesogenic diet on autonomic regulation in a rat model of chronic spinal cord injury Gregory D. Fink, Ph.D.

East Lansing, MI, USA

Chair: Satish Raj

Dunes I-III

9:45-10:00 AM

Coffee Break

Dunes Foyer

Course on Autonomic Medicine

Organizers: David Goldstein and Howard Snapper

The Dysautonomia Project is sponsoring the creation of a video education program for autonomic disorders in collaboration with the AAS

10:00 AM-1:00 PM

Dunes I-II

Parallel session

10:00-10:05 AM Introduction to The Dysautonomia Project 
10:05-1:00 PM Why are dysautonomias hard?

What is the ANS?

The ANS has parts

Organization of the ANS

Chemical messengers of the ANS

Homeostatis and the ANS

Stress and the ANS

Introduction to dysautonomias

The syndromic nature of dysautonomias

Autonomic function tests

Pediatric dysautonomias

Adult dysautonomias

Geriatric dysautonomias

Miscellaneous dysautonomias

Treatment of dysautonomias

\section{Research Testing of Autonomic Function Workshop}

Organizers: Amy Arnold and Matthew Lloyd

10:00 AM-12:30 PM

Dunes III

Parallel session

10:00-12:30 PM Assessment of blood pressure and heart rate in preclinical models

Amy Arnold, Ph.D.

Hershey, PA, USA

Measurement of sympathetic activity in preclinical models

Sean Stocker, Ph.D.

Pittsburgh, PA, USA

Cross-correlation analysis in the quantification of sympathetic outflow to muscle and skin in humans

Vaughan Macefield, Ph.D.

Melbourne, Australia

How to measure cardio-vagal and sympathetic baroreflex sensitivity in humans

Andre Diedrich, M.D., Ph.D.

Nashville, TN, USA

Catecholamines and cardiovascular autonomic neuropathies in humans

Giris Jacob, M.D., Ph.D.

Tel Aviv, Israel

Quantitative assessment of sympathetic structure and function in humans

Christopher Gibbons, M.D.

Boston, MA, USA

12:30-1:30 PM Lunch Break (on your own)

Clinical Autonomic Testing Workshop

Organizers: Wolfgang Singer and David Sletten

1:30-4:00 PM

Dunes III

1:30-4:00 PM Clinical autonomic testing - physiology and approach

Testing demonstration

Illustrative cases, pitfalls, and discussion

4:00-5:00 PM

Free Time

5:00-5:30 PM

Registration: Industry Dinner Symposium

Dunes I-III

5:30-7:15 PM

Industry Dinner Symposium: Alnylam Pharmaceuticals

Dunes I-III

7:15-7:30 PM

Coffee Break

Dunes Foyer

7:30-9:30 PM Poster Session I and Trainee Poster Competition

Dunes IV-V and Dunes Foyer 


\section{THURSDAY, NOVEMBER 7, 2019}

7:00-8:30 AM Continental Breakfast

Pelican/Heron

Session 1: Autonomic Regulation of Heart and Vasculature

Chairs: Kaushik Patel and Jacqueline Limberg

8:30-10:00 AM

Dunes I-II

Parallel session

8:30-8:45 AM

Evidence of adaptations in the neural control of body temperature following heat acclimation in humans H. Barry, G. Chaseling, S. Moreault, C. Sauvageau, P. Behzadi, H. Gravel, N. Ravanelli, D. Gagnon Montréal, Canada

8:45-9:00 AM Low-frequency sinusoidal electrical stimulation of the dorsolateral prefrontal cortex modulates muscle sympathetic nerve activity in humans

T. Dawood, L.A. Henderson, V.G. Macefield

Melbourne, Australia

9:00-9:15 AM Sympathetic vasodilation improves insulin-mediated microvascular recruitment in the forearm of obese human subjects J. Celedonio, J.N. Patel, A. Ruiz, E.C. Smith, S. Lonce, S. Paranjape, L. Okamoto, I. Biaggioni, A. Gamboa Nashville, TN, USA

9:15-9:30 AM Differences in regional grey matter volume of the brain are related to mean blood pressure and muscle sympathetic nerve activity in humans

S. Kobuch, R.H. Fatouleh, J.M. Macefield, L.A. Henderson, V.G. Macefield

Melbourne, Australia

9:30-9:45 AM Impact of age and sex on neural cardiovascular responsiveness to cold pressor test in humans

M.L. Keller-Ross, H.A. Cunningham, J.R. Carter

Minneapolis, MN, USA

9:45-10:00 AM Neuroplasticity in nucleus tractus solitarii after cardiovascular deconditioning induced by hindlimb unloading in rats L. Lima-Silveira, E.M. Hasser, D.D. Kline

Columbia, MO, USA

Symposium 1: Role of Autoimmunity in POTS

Sponsored by Dysautonomia International

Chairs: Steven Vernino and Xichun Yu

8:30-10:00 AM

Dunes III

Parallel session

8:30-8:35 AM

Introduction to Dysautonomia International

8:35-10:00 AM

Autoimmunity in POTS: overview

Steven Vernino, M.D., Ph.D.

Dallas, TX, USA

Overview of G protein coupled receptor antibodies in POTS

David C. Kem, M.D. and Xichun Yu, M.D.

Oklahoma City, OK, USA

Sjögren syndrome and POTS

Brent P. Goodman, M.D.

Scottsdale, AZ, USA

10:00-10:30 AM Coffee Break 
Session 2: Autonomic Neuropathy and POTS

Chairs: Howard Snapper and Meredith Bryarly

10:30 AM-12:00 PM

Dunes I-II

Parallel session

10:30-10:45 AM Low frequency variability of systolic arterial pressure accurately predicts autonomic injury during the acute phase of spinal cord injury

V.E.M. Lucci, J.A. Inskip, M.S. McGrath, B.K. Kwon, V.E. Claydon

Burnaby, Canada

10:45-11:00 AM Clinical phenotyping of postural tachycardia syndrome

B. Roschach, V. Galvis, A.I. Penzlin, I. Bonyhay, J. Celli, R. Freeman, C.H. Gibbons

Boston, MA, USA

11:00-11:15 AM Orthostatic intolerance in the young and the benefits of oral rehydration

M.S. Medow, K. Guber, S. Chokshi, C. Terilli, P. Visintainer, J.M. Stewart

Valhalla, $N Y$, USA

11:15-11:30 AM Hereditary transthyretin (hATTR) amyloidosis: symptoms and signs in early disease

C.H. Gibbons, A. Gonzalez-Duarte, F. Barroso, M. Campagnono, J. Garcia, S. Rajan, R. Freeman Boston, MA, USA

11:30-11:45 AM Putative genetic markers for postural tachycardia syndrome

J.R. Boris, H. Hakonarson

Philadelphia, PA, USA

11:45-12:00 PM Responsiveness of neuropathy symptom and change (NSC) score components in inotersen treatment of hereditary transthyretin amyloidosis polyneuropathy

P.J.B. Dyck, T. Coelho, M. Waddington Cruz, T. Brannagan, S. Khella, C. Karam, J.L. Berk, M.J. Polydefkis, J.C. Kincaid, J.F. Wiesman, W.J. Litchy, M.L. Mauermann, E.J. Ackermann, B.F. Baker, S.W. Jung, S.Guthrie, M. Pollock, P.J. Dyck Rochester, MN, USA

\section{Symposium 2: Sex Differences in Autonomic Control of Blood Pressure and Metabolism}

Chairs: Cyndya Shibao and Amanda Miller

10:30 AM-12:00 PM

Dunes III

Parallel session

10:30-12:00 PM Angiotensin-(1-7) and metabolic function in obesity: sex matters

Amy Arnold, Ph.D.

Hershey, PA, USA

Neural control of blood pressure during pregnancy in humans

Qi Fu, M.D., Ph.D.

Dallas, TX, USA

Racial difference in cholinergic modulation of oxidative stress and its clinical application in obese women

Cyndya Shibao, M.D.

Nashville, TN, USA

Compensatory interaction between sympathetic baroreflex sensitivity and vascular transduction in males but not females

C.E. Taylor, S.L. Hissen, R. Brown, V.G. Macefield

Sydney, Australia

Sympathetic and cardiovascular responses to acute mental stress in post-traumatic stress disorder (PTSD): sex differences I.T. Fonkoue, T.N. Jones, M. Vemulapalli, D. DaCosta, M.L. Kankam, J. Park

Atlanta, GA, USA

12:00-1:30 PM Poster Session II

(box lunch provided)

Dunes IV-V and Dunes Foyer

1:30-4:00 PM Free Time

4:00-5:30 PM Mentor-Mentee Round Table

Organizers: Amanda Miller and Elisabeth Golden

Pelican 


\section{5:30-6:00 PM Free Time}

6:00-7:00 PM Plenary Lecture 2: Streeten Plenary Lecture

Multiple system atrophy and brainstem control of survival

Eduardo E. Benarroch, M.D.

Rochester, MN, USA

Chair: Satish Raj

Dunes I-III

Session 3: Awards Presentations

Chairs: Satish Raj and Marvin Medow

7:00-8:30 PM

Dunes I-III

7:00-7:15 PM

FMS/Penaz Wesseling Travel Fellowship Award: Towards improved hemodynamic criteria to distinguish between classic orthostatic hypotension and reflex syncope during tilt-table testing M. Ghariq, F.I. Kerkhof, R.H.A.M. Reijntjes, R.D. Thijs, J.G. van Dijk

Leiden, The Netherlands

7:15-7:30 PM FMS/Penaz Wesseling Travel Fellowship Award: Effect of dietary salt intake on morning blood pressure surge in healthy women

R. Takeda, A.S. Stickford, J.-K. Yoo, R.A. Parker, M.A. Roberts-Reeves, Q. Fu Dallas, TX, USA

7:30-7:45 PM Streeten Travel Fellowship Award: Increased gastrointestinal gut hormones secretion following a glucose challenge in postural tachycardia syndrome

N.C. Breier, S.Y. Paranjape, S. Scudder, S. Lonce, S.E. Mehr, A. Diedrich, C.R. Flynn, C.A. Shibao

Nashville, TN, USA

7:45-8:15 PM The Felicia Axelrod Award

8:15-8:30 PM Recognition of Fellows of the American Autonomic Society

FRIDAY, NOVEMBER 8, 2019

6:15-6:45 AM Registration: Industry Breakfast Symposium

Dunes I-III

6:45-8:00 AM Industry Breakfast Symposium: Akcea Therapeutics

Dunes I-III

8:00-9:00 AM Plenary Lecture 3: Hot Topic Plenary Lecture

The gut microbiome: all hype or true hope?

Amir Zarrinpar, M.D., Ph.D.

La Jolla, CA, USA

Chair: Satish Raj

Dunes I-III

9:00-9:15 AM

Coffee Break

Session 4: Orthostatic Hypotension and Supine Hypertension

Sponsored by Theravance Biopharma

Chairs: William Farquhar and Mitchell Miglis

9:15-10:50 AM

Dunes I-II

Parallel session

9:15-9:30 AM A phase 2 study of the efficacy, durability, and safety of ampreloxetine (TD-9855), a norepinephrine reuptake inhibitor, given once-daily to treat neurogenic orthostatic hypotension $(\mathrm{nOH})$ in subjects with synucleinopathies

H. Kaufmann, I. Biaggioni, A. Panneerselvam, B. Haumann, R. Vickery

New York, $N Y$, USA

9:30-9:45 AM

Local passive heat in the treatment of nocturnal supine hypertension in autonomic failure

L.E. Okamoto, J.E. Celedonio, E.C. Smith, A. Gamboa, C.A. Shibao, A. Diedrich, S. Paranjape, B.K. Black, J.A. Muldowney 3rd, A.C. Peltier, R. Habermann, C.G. Crandall, I. Biaggioni Nashville, TN, USA

9:45-10:00 AM Changes in supine blood pressure with long-term droxidopa use

L.A. Hewitt, S. Gorny, A. Lindsten, M. Karnik-Henry, S. Kymes, A. Favit

Deerfield, IL, USA 
10:00-10:15 AM Best Basic Science Autonomic Research: Bezold-Jarisch reflex and beyond: differential engagement of inhibitory and excitatory cardiopulmonary reflexes by capsaicin and phenylbiguanide R.A. Larson, M.W. Chapleau Iowa City, IA, USA

10:15-10:30 AM Can urine sodium concentration be determined through chloride test strips? N.D. Heeney, B.C.D. Hockin, S. Sanatani, K. Armstrong, V.E. Claydon Burnaby, Canada

10:30-10:50 AM Presentations from top 4 posters (4@ 5 min each)

\section{Symposium 3: Vasovagal Syncope: The Present and the Future} Chairs: Satish R. Raj and Juan Guzman

9:15-10:50 AM

Dunes III

Parallel session

9:15-10:50 AM Drugs for vasovagal syncope: what are the data?

Satish R. Raj, M.D.

Calgary, Canada

Pacemakers for vasovagal syncope

Dan Sorajja, M.D.

Scottsdale, AZ, USA

Can we ablate away vasovagal syncope?

Phang Boom Lim, Ph.D.

London, $U K$

Syncope in the young: what do we know about their physiology so far?

Mohammed Numan, M.D.

Houston, TX, USA

10:50-11:30 AM

Coffee Break

Symposium 4: Heart Failure: Cutting Edge Autonomic Approach

Chairs: Mark W. Chapleau and Amanda Peltier

11:30 AM-1:00 PM

Dunes I-II

Parallel session

11:30-1:00 PM Carotid body contribution to autonomic disorders

Rodrigo Iturriaga, Ph.D.

Santiago, Chile

Molecular basis of cardiac mechanosensory and chemosensory signaling: implications in heart failure

Mark W. Chapleau, Ph.D.

Iowa City, IA, USA

Splanchnic modulation in heart failure

Marat Fudim, M.D.

Durham, NC,USA

Symposium 5: Gastrointestinal System in Autonomic Disorders

Chairs: Gisela Chelimsky and Qi Fu

11:30 AM-1:00 PM

Dunes III

Parallel session 
11:30-1:00 PM Enteric nervous system: the big brain!

Keith A. Sharkey, Ph.D.

Calgary, Canada

Evaluation of GI problems in dysautonomia

Gisela Chelimsky, M.D.

Milwaukee, WI, USA

Management pearls for GI problems in autonomic disorders

John E. Fortunato, Jr., M.D.

Evanston, IL, USA

1:00-7:00 PM Free Time/Lunch On Your Own

1:30-3:30 PM AAS Board Meeting

Ibis

3:30-4:30 PM Clinical Autonomic Research Editorial Board Meeting

Ibis

4:30-5:30 PM AAS Committee Meetings

Pelican/Heron

7:00-10:00 PM Presidential Dinner

Pool Deck

SATURDAY, NOVEMBER 9, 2019

6:30-8:00 AM Continental Breakfast

Pelican/Heron

8:00-8:45 AM Plenary Lecture 4: MSA Plenary Lecture

The early stages of synculeinopathies: is prodromal MSA detectible?

Ronald Postuma, M.D.

Montreal, Canada

Chair: Satish Raj

Dunes I-III

Session 5: Multiple System Atrophy and Other Neurodegenerative Diseases

Sponsored by MSA Coalition

Chairs: Janice Gilden and Jose-Alberto Palma

Dunes I-III

8:45-10:05 AM

8:45-8:50 AM

8:50-9:05 AM

Introduction to MSA Coalition

Don Summers Memorial MSA Travel Award: Baseline characteristics of patients with multiple system atrophy enrolled in the Natural History Study of the Synucleinopathies

M.A. Perez, J.-A. Palma, L. Norcliffe-Kaufmann, W. Singer, P. Low, M.T. Pellecchia, H.-J. Kim, C. Shibao, A. Peltier, I. Biaggioni, D. Giraldo, M.J. Marti, A. Fanciulli, C. Terroba, M. Merello, D.S. Goldstein, R. Freeman, C.H. Gibbons, S. Vernino, F. Krismer, G. Wenning, H. Kaufmann; on behalf of the Natural History Study of the Synucleinopathies Study Group

New York, NY, USA

9:05-9:20 AM

Predicting evolution in pure autonomic failure

E.A. Coon, S.E. Berini, J.N. Mandrekar, E.E. Benarroch, P. Sandroni, P.A. Low, W. Singer

Rochester, MN, USA

9:20-9:35 AM Cutaneous biomarkers in multiple system atrophy

N. Wang, S. Rajan, D. Kern, J.A. Palma, H. Kaufmann, R. Freeman, C.H. Gibbons

Boston, MA, USA

9:35-9:50 AM Long-term administration of intrathecal mesenchymal stem cells in multiple system atrophy - a compassionate use experience

W. Singer, A.B. Dietz, A.D. Zeller, T.L. Gehrking, J.D. Schmelzer, D.M. Sletten, J.A. Gehrking, A.M. Schmeichel, E.A. Coon, P. Sandroni, E.E. Benarroch, J.Y. Matsumoto, J.H. Bower, A. Hassan, A. McKeon, P.A. Low

Rochester, MN, USA 
9:50-10:05 AM Autonomic changes in Parkinson's disease patients treated with L-threo-3,4-dihydroxyphenylserine and high dose Carbidopa therapy

K.R. Hay, K.E. McDonell, P. Trujillo, C. Shibao, S. Paranjape, O. Roman, D.O. Claassen

Nashville, TN, USA

10:05-10:30 AM Coffee Break

10:30 AM-12:30 Case-Based Discussion: Ultimate Autonomic Challenge

$\mathrm{PM}$

Organizer: Juan Guzman

Dunes I-III

12:30-1:00 PM AAS Business Meeting and Closing Remarks

Dunes I-III

2:00-3:00 PM

Registration: Patient Seminar: Updates from the 2019 AAS Meeting

3:00-8:00 PM

Patient Seminar: Updates from the 2019 AAS Meeting

Organized by Dysautonomia International

Dunes I-II

(separate registration required)

\section{POSTER SESSION I AND TRAINEE POSTER COMPETITION* \\ WEDNESDAY, NOVEMBER 6, 2019 \\ 7:30-9:30 PM \\ Dunes IV-V and Dunes Foyer}

*Abstracts with asterisks are included in the Trainee Poster Competition

Autonomic Failure

Poster \#1*

Addressing orthostatic hypotension in Parkinson disease patients in a neurology resident continuity clinic: one year followup

E.P. Golden, T. Le, B. Bleiberg, L.S. Brown, S. Khan, S. Vernino, P. Khemani

Dallas, TX, USA

Poster \#2* Disease stage and UMSARS progression in patients with multiple system atrophy enrolled in the Natural History Study of the Synucleinopathies: implications for clinical trials

M.A. Perez, J.-A. Palma, L. Norcliffe-Kaufmann, W. Singer, P. Low, M.T. Pellecchia, H.-J. Kim, C. Shibao, A. Peltier, I. Biaggioni, D. Giraldo, M.J. Marti, A. Fanciulli, C. Terroba, M. Merello, D.S. Goldstein, R. Freeman, C.H. Gibbons, S. Vernino, F. Krismer, G. Wenning, H. Kaufmann; on behalf of the Natural History Study of the Synucleinopathies Study Group

New York, NY, USA

Poster \#3* Symptoms of orthostatic hypotension S. Rajan, J. Celli, I. Bonyhay, C.H. Gibbons, R. Freeman Boston, MA, USA

Poster \#4 Conjugal multiple system atrophy: chance, shared risk factors, or evidence of infectivity? E.A. Coon, W. Rocca, J.E. Ahlskog, J.Y. Matsumoto, P.A. Low, W. Singer

Rochester, MN, USA

Poster \#5* Long-term trends in myocardial sympathetic innervation and function in synucleinopathies G. Lamotte, C. Holmes, T. Wu, D.S. Goldstein Bethesda, MD, USA

Poster \#6* Reliable indicators of autonomic function test in Parkinson's disease B.A. Yoon, J.K. Kim, J.W. Kim, S.M. Cheon Busan, South Korea

Poster \#7 Post-mortem and in vivo evidence for "sick but not dead" central dopaminergic neurons in multiple system atrophy: implications for neurotrophic factor gene enhancement therapy D.S. Goldstein, P. Sullivan, C. Holmes, K. Bankiewicz

Bethesda, MD, USA

Poster \#8* Longitudinal changes in autonomic symptom burden predict decline in activities of daily living in Parkinson's disease M. Sklerov, C. Shih, N. Browner, J.-A. Palma, E. Dayan

Chapel Hill, NC, USA

Poster \#9* Submandibular gland alpha-synuclein deposition occurs without local sympathetic denervation or dysfunction in Parkinson disease

R. Isonaka, P. Sullivan, D.S. Goldstein

Bethesda, MD, USA 
Genetic Autonomic Disorders

Poster \#10* Colocalization of alpha-synuclein with tyrosine hydroxylase in skin biopsies from individuals at genetic risk of Parkinson disease: a genotype-phenotype study

R. Isonaka, D. Narendra, D.S. Goldstein

Bethesda, MD, USA

Poster \#11* A case of indifference to pain and central anhidrosis

K. Shouman, K.V. Minota Pacheco, A.M. Schmeichel, T.L. Gehrking, J.A. Gehrking, A.D. Zeller, P.A. Low, W. Singer Rochester, MN, USA

Poster \#12* Neurogenic bladder dysfunction in patients with familial dysautonomia: a cross-sectional study

B.J. Balgobin, J.-A. Palma, B. Peyronnet, B. Brucker, H. Kaufmann

New York, $N Y$, USA

Poster \#13* Frequency of seizures and epilepsy in familial dysautonomia

B.J. Balgobin, M.A. Perez, J.-A. Palma, L. Norcliffe-Kaufmann, H. Kaufmann

New York, NY, USA

Autonomic Regulation

Poster \#14 Does renal denervation restore GABAergic tone in the paraventricular nucleus and thus attenuate the enhanced activation of the PVN in heart failure?

K.P. Patel, K. Katsurada, X. Liu, H. Zheng

Omaha, NE, USA

Poster \#15* Role of arterial baroreflex in respiratory sinus arrhythmia: a study using oscillatory lower body negative pressure in human subjects

A. Sood, S. Srivastav, D.S. Chandran, A.K. Jaryal, K.K. Deepak

New Delhi, India

Poster \#16* The effect of dietary sodium intake on central blood pressure variability in young adults

K.U. Migdal, J.C. Watso, M.C. Babcock, A.T. Robinson, M.M. Wenner, S.D. Stocker, W.B. Farquhar

Newark, DE, USA

Cardiovascular Disease, Obesity \& Aging

Poster \#17* Relationships between cardiovascular disease, autonomic function, neuropathic pain, and depression following spinal cord injury

M.C. Dorton, S. de Groot, M. Post, J.J. Cragg, J.K. Kramer, V.E. Claydon

Burnaby, BC, Canada

Poster \#18 Slow breathing training reduces blood pressure even in healthy normotensive subjects

E.C. Smith, S. Paranjape, K. Nelson, A. Diedrich, G. Birdee, A. Gamboa

Nashville, TN, USA

Diabetic, Autoimmune \& Other Autonomic Neuropathies

Poster \#19* Granulomatosis with polyangiitis presenting as autonomic failure

B. Blech, M. Grill, B. Goodman

Scottsdale, AZ, USA

Poster \#20 Quality improvement and practice-based research in diabetic autonomic neuropathy: preliminary observations A. Barboi, O. Kincaid, S. Pocica, S. Tideman, L. Garduno, V. Patel, L. Hillman, D. Macapinlac, R. Frigerio, K.C. Simon, D. Maraganore

Evanston, IL, USA

Poster \#21* Clinical features of autoimmune gastrointestinal dysmotility in Japan

A. Mukaino, S. Nakane, H. Minami, H. Isomoto, O. Higuchi, E. Ihara, A. Ido, H. Matsuo, K. Nakao, T. Yamashita, Y. Ando Kumamoto, Japan

Exercise, Temperature Regulation \& Hypoxia

Poster \#22 Quantification of vasodilation and sweating responses during heat stress in a person with and without spinal cord injury: a case report

M.T. Trbovich, R.C. Camacho

San Antonio, TX, USA

Poster \#23* Is there an association between resting heart rate and cardiovagal function early-to-late phases of recovery after resistance training?

G.L. Garcia, C.J.G. Cruz, E.M.K.V.K. Soares, M.T. Morlin, L.F. Junqueira Jr., L.G.G. Porto, G.E. Molina

Brasília, Brazil 
Poster \#24* Evolution of pulmonary hemodynamics during severe sustained hypoxia

F. Hoffmann, U. Limper, V. Zaha, H. Reuter, L. Zange, J. Schulz-Menger, M. Hein, H. Sadek, S. Baldus, B. Levine, J. Jordan, J. Tank

Cologne, Germany

Microneurography \& Cardiovascular Reflexes in Humans

Poster \#25* Early-onset blood pressure and muscle sympathetic nerve activity responsiveness to cold pressor test in young, healthy adults

H.A. Cunningham, J.R. Carter

Houghton, MI, USA

Poster \#26* Cardiorespiratory response to the exercise pressor reflex in women through the menstrual cycle with or without oral contraceptive use

I. Ivry, E. Assadpour, S. Wasef, B. Adeyinka, H. Edgell

Toronto, ON, Canada

Poster \#27* Effects of obstructive sleep apnea on baroreflex gain in patients with hypertrophic cardiomyopathy

S. Venkataraman, A. Rajendran, J. Bukartyk, N. Covassin, J.B. Geske, M. Mansukhani, A.C. Chahal, V.K. Somers Rochester, MN, USA

Neuroimaging in Brain \& Heart

Poster \#28 HIV-related deficits in volume of the central autonomic network and resting cardio-autonomic tone R. McIntosh, J. Paparozzi, D. Qadir, D. Dukenik, G. French, J. Lobo Miami, FL, USA

Poster \#29* Specific cerebral multiple sclerosis lesions alter cardiovascular autonomic modulation

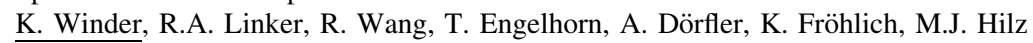
Erlangen, Germany

Orthostatic Hypotension \& Syncope

Poster \#30 Artificial intelligence algorithms and natural language processing for the recognition of syncope patients in emergency department medical records

F. Dipaola, M. Gatti, D. Shiffer, V. Pacetti, A.G. Bottaccioli, M. Minonzio, R. Menè, M. Solbiati, G. Costantino, M. Anastasio, E. Sini, F. Barbic, E. Brunetta, R. Furlan

Milan, Italy

Poster \#31* Forearm vascular resistance responses to the Valsalva maneuver B.C.D. Hockin, E.Z. Tang, M.G. Lloyd, V.E. Claydon

Burnaby, BC, Canada

Poster \#32 Patient-reported "good" days during a prospective study of the treatment of neurogenic orthostatic hypotension with droxidopa

S. Kymes, L.A. Hewitt, C. François

$\overline{\text { Deerfield, }}$ IL, USA

Poster \#33* Midodrine for the prevention of tilt-induced and clinical vasovagal syncope: A systematic review and meta-analysis L. Lei, S.R. Raj, R.S. Sheldon

Calgary, AB, Canada

Poster \#34 Elective EEG monitoring combined with head-up tilt in the diagnosis of syncope and non-syncopal fainting J.A.S. Muldowney 3rd, P. Sharma, L.E. Okamoto, H.H. Sonmezturk, A. Peltier, C.A. Shibao, I. Biaggioni Nashville, Tennessee, USA

Poster \#35 In their own words: a qualitative analysis of patients' experience of living with syncope and POTS M. Runte, T. Runte, S. Jarvie, D. Basil, S. Bedard, D. Porter, K. Williams-Whitt Lethbridge, AB, Canada

Poster \#36 Initial orthostatic hypotension causes transient postural tachycardia J.M. Stewart, T. Fialkoff, B. Tuma-Marcella, P. Visintainer, C. Terilli, M.S. Medow $\overline{\text { Valhalla, } N Y}$, USA

Pediatric Autonomic Disorders

Poster \#37* IVIG for treatment of acquired pseudo-obstruction of unknown etiology N. Rademacher, T. Chelimsky, M. Sood, R. Kliegman, D. Basel, G. Chelimsky Milwaukee, WI, USA

Poster \#38 Concurrent encephalopathy and autonomic failure in a child with positive ganglionic nicotinic acetylcholine receptor antibodies

G.A. Cook, J.L. Jaskiewicz

Bethesda, MD, USA 
Poster \#39 Gastrointestinal (GI) symptoms and GI biomarkers in postural tachycardia syndrome (POTS) and cardiogenic syncope (NCS) in adolescents and young adults

M.T. Numan, D. Halawa, I.J. Butler, H. Zamil

Houston, TX, USA

Poster \#40 Breathing disturbances in a child with Rett syndrome

H.W. Choi, P. Pianosi

Minneapolis, MN, USA

Postural Tachycardia Syndrome (POTS)

Poster \#41 Postural tachycardia syndrome in a family with pseudoxanthoma elasticum A. Arvantaj, C. Geiger, J. Robinson, B. Katirji

Cleveland, $\mathrm{OH}, \mathrm{USA}$

Poster \#42 Synergistic but separable sensory changes in migraineurs and postural tachycardia syndrome M.M. Cortez, L. Millsap, K.C. Brennan Salt Lake City, UT, USA

Poster \#43* Body compression in postural tachycardia syndrome improves orthostatic tolerance in a dose-dependent pattern K.M. Bourne, R.S. Sheldon, D.V. Exner, J. Tyberg, K. Kogut, J. Ng, N. Sheikh, M. Lloyd, E. Scott, S.R. Raj Calgary, $A B$, Canada

Poster \#44* Diagnosis of postural tachycardia syndrome: a comparison between the tilt and active stand tests V. Galvis, A.I. Penzlin, B. Roschach, S. Rajan, J. Celli, I. Bonyhay, R. Freeman, C.H. Gibbons Boston, MA, USA

Poster \#45* Cognitive function is impaired during active standing in postural tachycardia syndrome A.J. Miller, T. Sheehan, M. Feeley, K. Bourne, A.C. Arnold

Hershey, PA, USA

Poster \#46* Prevalence of hypermobile Ehlers-Danlos syndrome in postural tachycardia syndrome A.J. Miller, L.E. Stiles, T. Sheehan, R. Bascom, H.P. Levy, C. Francomano, A.C. Arnold Hershey, PA, USA

Poster \#47 Immunotherapy as an effective treatment of POTS with functionally active adrenergic and muscarinic antibodies after human papillomavirus vaccination: a case report A.T. Del Pozzi, G. Wallukat, I. Schimke, S. Blitshteyn Muncie, IN, USA

Poster \#48* Natural history of postural tachycardia syndrome A.I. Penzlin, B. Roschach, A. Benson, V. Galvis, I. Bonyhay, C.H. Gibbons, R. Freeman Boston, MA, USA

Poster \#49* Effects of transcutaneous vagal nerve stimulation on orthostatic tolerance in patients with postural tachycardia syndrome (POTS)

D. Shiffer, R. Furlan, F. Barbic, M. Minonzio, B. Cairo, A. Porta, N. Montano, E. Tobaldini, L. Furlan, V. Urechie, I. Biaggioni, A. Diedrich

Milan, Italy

Poster \#50 A double-blind, placebo-controlled, crossover pilot trial of gabapentin for treatment of postural tachycardia symptoms R.K. Khurana

\section{Baltimore, $M D$, USA}

Poster \#51* Cerebral blood flow, autoregulation, and symptoms during orthostasis with lower body compression in postural tachycardia syndrome M.G. Lloyd, K.M. Bourne, R.S. Sheldon, D.V. Exner, J. Tyberg, K. Kogut, J. Ng, N. Sheikh, E. Scott, S.R. Raj Calgary, $A B$, Canada

Sympathovagal Balance \& Spectral Analysis

Poster \#52* Norepinephrine transporter inhibition with atomoxetine: autonomic effects during orthostasis in patients with vasovagal syncope M.G. Lloyd, L.Lei, J.C. Guzman, T. Kus, F.A. Ayala-Paredes, R.S. Sheldon, S.R. Raj

$\overline{\text { Calgary, } A B}$, Canada 


\section{POSTER SESSION II \\ THURSDAY, NOVEMBER 7, 2019}

12:00-1:30 PM

Dunes IV-V and Dunes Foyer

Neuroimaging in Brain \& Heart

Poster \#53 Cannabis induces changes in functional brain connectivity that correlate with increased vagal tone and clinical analgesia in chronic neuropathic pain

H. Sharon, L. Weizman, J. Espaniol, L. Dayan, G. Jacob

Poster \#54 Peripheral chemoreflex drives sympathetic activation in the human brainstem and hypothalamus

D.A. Gerlach, J. Manuel, A. Hoff, H. Kronsbein, F. Hoffmann, K. Heusser, A. Diedrich, H. Ehmke, J. Jordan, F. Beissner, J. $\underline{\text { Tank }}$

Poster \#55 Functional connectivity of the central autonomic network and regulation of parasympathetic tone in health and chronic disease

R. McIntosh, K. Reaves, G. French, J. Lobo, C. Chang

Miami, FL, USA

\section{Autonomic Failure}

Poster \#56 HIV-related decrement in respiratory arrhythmia during a deep breathing test

R. McIntosh, K. Reaves, D. Dukenik, J. Lobo, V. Sookdeo

Miami, FL, USA

Poster \#57 Normal values of quantitative thermoregulatory sweat testing (Q-TST)

A. Arvantaj, J. Robinson, C. Geiger, B. Katirji

Cleveland, $\mathrm{OH}, \mathrm{USA}$

Poster \#58 Experience from a multidiscliplinary care clinic for multiple system atrophy

S. Vernino, E. Golden, E. Kent, G. Huet, M. Bryarly

Dallas, TX, USA

Poster \#59 Neurogenic orthostatic hypotension in patients referred for evaluation in a Chilean autonomic clinic

J. Idiaquez, O. Trujillo, J.F. Idiaquez, R. Iturriaga

Santiago, Chile

Poster \#60 Cutaneous alpha-synuclein deposition in idiopathic REM sleep behavioral disorder

M.G. Miglis, J. Zitser, S. Rajan, E. During, R. Freeman, C.H. Gibbons

Stanford, CA, USA

Poster \#61 Sudomotor abnormalities in idiopathic REM sleep behavior disorder

J. Zitser, S. Muppidi, D.I. Sinn, E. During, T. Prieto, S. Jaradeh, M.G. Miglis

San Francisco, CA, USA

Poster \#62 Anti-cholinergic receptor antibodies, cardiovascular hemodynamics and dysautonomia symptoms relationship in pure autonomic failure: a two-year follow-up

F. Barbic, M. Minonzio, B. Cairo, D. Shiffer, E. Brunetta, F. Dipaola, M. Folci, R. Fornerone, F. Andreetta, C. Antozzi, R. Mantegazza, A. Porta, R. Furlan

Milan, Italy

Poster \#63 A phase-2 randomized placebo-controlled trial of sirolimus in multiple system atrophy: protocol, recruitment and preliminary adverse event profile

J.-A. Palma, J. Martinez, S. Simon, L. Norcliffe-Kaufmann, H. Kaufmann

New York, NY, USA

Poster \#64 Impact of spinal cord injury on maximal cutaneous vasodilation, myogenic and venoarteriolar responses M. Trbovich, Y. Wu, W. Koek, J. Zhao, A. McMahon, D. Kellogg

San Antonio, TX, USA

Poster \#65 Acute effects of mechanical somatosensory stimulation on cardiac autonomic control and its relationship with postural control in patients with Parkinson's disease

B.M. Franco, N.R. Marques, V.C. Moreno, M.H. Kuroda, F.A. Barbieri, A.R. Zamunér

São Paulo, Brazil

\section{Cardiac Autonomic Innervation}

Poster \#66 Multiple functional abnormalities in cardiac sympathetic nerves revealed by a comprehensive computational model in Lewy body diseases

D.S. Goldstein, M. Pekker, G. Eisenhofer, Y. Sharabi

Bethesda, MD, USA

Poster \#67 The relationship between sympathetic modulation and central blood pressure in African American and Caucasian women P. Latchman, G. Gates, L. Mangone, T. Yue, R. Axtell, Q. Yang, M. Latchman, H. Zhang, J. Pereira, R. De Meersman New Haven, $C T$, USA 
Diabetic, Autoimmune \& Other Autonomic Neuropathies

Poster \#68 Autonomic dysfunction and its association with painful and painless neuropathy R. Lüddecke, R. Baron, J. Gierthmühlen

Kiel, Germany

Poster \#69 Dysautonomia in Guillain-Barré syndrome: an under-recognized and potentially life-threatening phenomenon A.V. Varma-Doyle, H. Gould

New Orleans, LA, USA

Genetic Autonomic Disorders

Poster \#70 Hereditary sensory and autonomic neuropathy type IV: case-report and review of immune dysfunction in the disease A.V. Varma-Doyle, M. Marble, L. McBride, A. Tilton

New Orleans, LA, USA

Poster \#71 Familial dysautonomia and congenital adrenal hyperplasia: one patient with two rare genetic disorders B.J. Balgobin, J.-A. Palma, L. Norcliffe-Kaufmann, H. Kaufmann

New York, $N Y$, USA

Autonomic Regulation

Poster \#72 Methionine sulfoxide reductase deficiency exacerbates metabolic dysfunction in mice fed a high-fat diet: implications in metabolic syndrome

R. Sabharwal, L. Yang, M.W. Chapleau

Iowa City, IA, USA

Poster \#73 Angiotensin II type 2 receptors restore autonomic function and delay onset of dilated cardiomyopathy in male mice with muscular dystrophy

L. Yang, K. Zimmerman, R.M. Weiss, R. Sabharwal

Iowa City, IA, USA

Cardiovascular Disease, Obesity \& Aging

Poster \#74 Effect of the menstrual cycle on resting, exercise and post-exercise heart rate in healthy women C. Giovanna, G. Porto Luiz Guilherme, M. Daniel, S. Edgard, G. Giliard, C. Carlos Janssen, E. M. Guilherme Brasília, Brazil

Poster \#75 Sympathetic neural traffic in older patients with type 2 diabetes mellitus

K. Heusser, J. Tank, B. Johannes, J. Jordan

Cologne, Germany

Autonomic Clinical Practice

Poster \#76 Autonomic medical practice as sampled by physician-rating websites

W.P. Cheshire

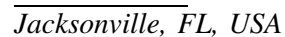

Exercise, Temperature Regulation \& Hypoxia

Poster \#77 Fast heart rate recovery and its association with vagal withdraw after active orthostatic test in men G.E. Molina, C.J.G. Cruz, M.T. Morlin, E.M.K.V.K. Soares, G.L. Garcia, L.G.G. Porto Brasília, Brazil

Poster \#78 Acute effects of propranolol and pindolol on heart rate, metabolic rate, and cold-induced thermogenesis in lean, young men R.J. Brychta, R. Muniyappa, S. McGehee, S. Huang, J.D. Hattenbach, S.L. Bell, A.B. Courville, S. Yang, B.P. Leitner, C.J. Duckworth, T. Cassimatis, L.A. Fletcher, H. Sasai, A.M. Cypess, M.L. Reitman, K.Y. Chen Bethesda, MD, USA

Poster \#79 Analysis of the speed of post-exercise heart rate recovery in athletes who practice triathlon and crossfit M.T. Morlin, C.J.G. Cruz, E.M.K.V.K. Soares, L.G.G. Porto, G.E. Molina Brasília, Brazil 
Gastrointestinal \& Urogenital Systems, Ibs, Cystitis

Poster \#80 Neurotensin-induced relaxation of the guinea pig ileal circular muscle layer is indirect and mediated by release of vasoactive intestinal peptide

H. Wang, D.M. Kendig, K.S. Murthy, J.R. Grider

Baltimore, MD, USA

Microneurography \& Cardiovascular Reflexes in Humans

Poster \#81 Consistent first trimester elevations in muscle sympathetic nerve activity and sympathetic baroreflex sensitivity between first and second pregnancy: a case study

C.E. Taylor, S. Kobuch, S.L. Hissen, D. Boulton, A. Burton, N. Kramadhari, S. Dayaratne, V.G. Macefield

Sydney, Australia

Poster \#82 Reproducibility of valsalva maneuver derived baroreflex parameters

C. Ustine, L. Conant, P. Simpson, G. Chelimsky, C. O’Hara, T. Chelimsky

Milwaukee, WI, USA

Orthostatic Hypotension \& Syncope

Poster \#83 A case of dysautonomia in post-craniectomy syndrome of the trephined

M. Beeler, T. Malone, J. Boulter, R. Bell, M. Rosner, G. Cook

Bethesda, MD, USA

Poster \#84 Assessment of practice patterns among neurologists and cardiologists for the care of patients with neurogenic orthostatic hypotension

T. Finnegan, J. Maeglin

New York, NY, USA

Poster \#85 Prevention of vasovagal syncope using hyoscyamine

H. Snapper, M. Jawaid

Marietta, GA, USA

Poster \#86 Norepinephrine transporter inhibition for the prevention of tilt-induced vasovagal syncope: a systematic review and metaanalysis

L. Lei, A. Qaddoura, S.R. Raj, R.S. Sheldon MD

Calgary, AB, Canada

Poster \#87 Does recent weight loss predispose to vasovagal syncope? a case-control series

B. Cumming, D. Jardine

Christchurch, New Zealand

Poster \#88 Factors influencing patients' decision making around accessing emergency medical services for syncope

T. Runte, T. Williamson, R. Sheldon, T. Campbell, K. King-Shier, S.R. Raj, M. Runte

Lethbridge, AB, Canada

Postural Tachycardia Syndrome (POTS)

Poster \#89 Effect of a neck compression collar on cardiorespiratory function in postural tachycardia syndrome (POTS)

M. Nardone, J. Guzman, P. Harvey, J. Floras, H. Edgell

Toronto, ON, Canada

Poster \#90 T cell surface marker, CRTH2 expression in postural tachycardia syndrome

H.A. Abdallah, H.M. Abdallah, C. Kim, O. Alpan

McLean, VA, USA

Poster \#91 Sleep disturbances in postural tachycardia syndrome

T. Fayyaz, A. Sheikh, S.B. Alam, M.Y. Bilal, H. Nazeer, M. Farooq, S. Shaheen, H. Mistry, S. Usmani, N. Chaudhary, W. Almardini, A. Suleman

Dallas, TX, USA

Poster \#92 Acute modafinil and cognition in postural tachycardia syndrome

A.C. Arnold, K. Haman, E.M. Garland, A.J. Miller, M. Wang, B. Shen, S.Y. Paranjape, B.K. Black, A. Diedrich, D. Robertson, C.A. Shibao, I. Biaggioni, S.R. Raj

Hershey, PA, USA

Poster \#93 A new disease cluster: postural tachycardia syndrome, hypermobile Ehlers-Danlos syndrome/hypermobility spectrum disorder and mast cell activation syndrome

I. Cheung, J. Holoff, J. Guzman, R.M. Londono, S. Walsh, P. Vadas

Toronto, ON, Canada

Poster \#94 Prevalence of anxiety and depression in children with postural tachycardia syndrome: a retrospective study B. Kakavand, A. Centner, S. Centner, S. Hasan

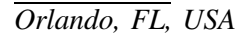


Poster \#95 Why do patients wear a compression garment for their POTS symptoms (or not)?

K.M. Bourne, R.S. Sheldon, S.R. Raj, M. Runte

Calgary, $A B$, Canada

Poster \#96 Autoimmunity and immunotherapy in postural tachycardia syndrome

K. Sato, A. Yamaga, Y. Nishimura, K. Shibata, T. Nakaoka, Y. Sunami, T. Hirai, T. Nakane, H. Sakura

Tokyo, Japan

Poster \#97 Efficacy of epidural blood patch as a treatment for chronic orthostatic intolerance: a case series

\section{Kinsella}

St. Louis, MO, USA

Poster \#98 Family inheritance in postural tachycardia syndrome: insights from a cross-sectional community-based survey

L.E. Stiles, K. Bourne, C.A. Shibao, L.E. Okamoto, E.M. Garland, A. Gamboa, A. Peltier, A. Diedrich, I. Biaggioni, R.S. Sheldon, S.R. Raj

East Moriches, NY, USA

Poster \#99 Somatic and autonomic findings in cancer related base of the skull syndromes A. Barboi, S. Pocica, S. Moffat, I. Kafkas, V. Patel

Evanston, IL, USA

Poster \#100 Efficacy and safety of droxidopa for the treatment of patients with symptomatic neurogenic orthostatic hypotension during 12 weeks of open-label treatment in a phase 4 study S. Gorny, L.A. Hewitt, A. Lindsten, S. Kymes, A. Favit

Deerfield, IL, USA

Poster \#101 A phase 2 dose-escalation study of ampreloxetine (TD-9855), a norepinephrine reuptake inhibitor, given once-daily to treat neurogenic orthostatic hypotension $(\mathrm{nOH})$ in subjects with synucleinopathies

H. Kaufmann, I. Biaggioni, A. Panneerselvam, B. Haumann, R. Vickery

New York, $N Y$, USA

\section{ACCME Accreditation Statement:}

This activity has been planned and implemented in accordance with the accreditation requirements and policies of the Accreditation Council for Continuing Medical Education through the joint sponsorship of the Medical College of Wisconsin and the American Autonomic Society. The Medical College of Wisconsin is accredited by the ACCME to provide continuing medical education for physicians.

\section{AMA Credit Designation Statement:}

The Medical College of Wisconsin designates this Live Activity for a maximum of 20.5 AMA PRA Category 1 Credits $^{\text {TM }}$. Physicians should claim only the credit commensurate with the extent of their participation in the activity. 
The American Autonomic Society would like to thank the following for their support of this meeting:

\section{CORPORATE YEAR-ROUND PARTNER}

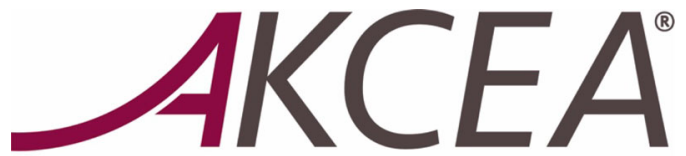

THERAPEUTICS

\section{PLATINUM SPONSOR}
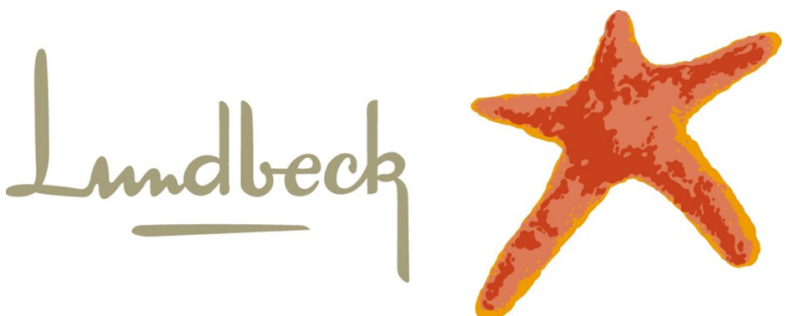

\section{GOLD SPONSORS}
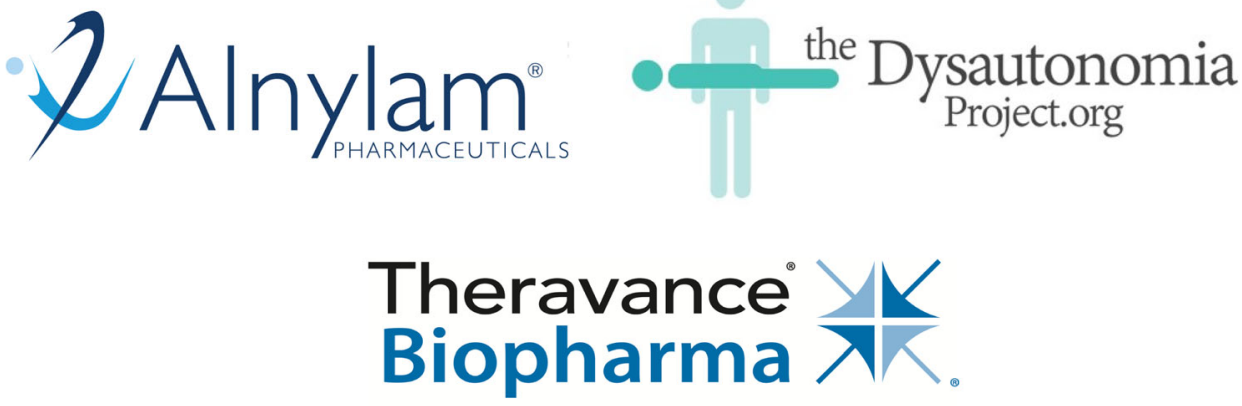

Medicines That Make a Difference ${ }^{\circ}$ 


\section{SYMPOSIA SPONSORS}
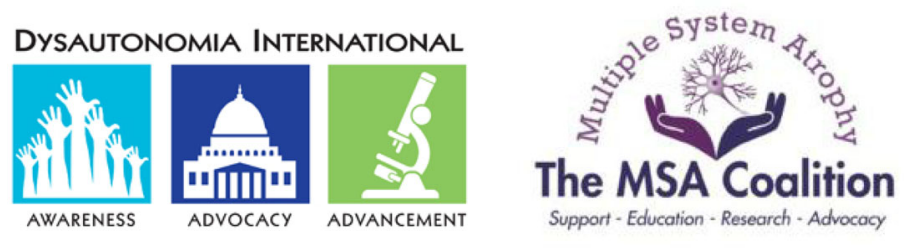

Theravance Biopharma

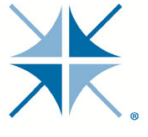

Medicines That Make a Difference ${ }^{\circ}$

\section{TRAVEL AWARD SPONSORS}

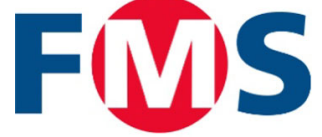

Finapres Medical Systems

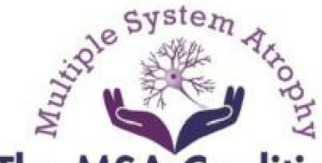

The MSA Coalition

Support - Education - Research-Advocacy

\section{INDUSTRY-SPONSORED SATELLITE SYMPOSIA}

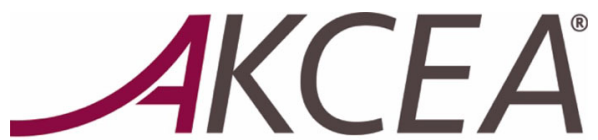

THERAPEUTICS

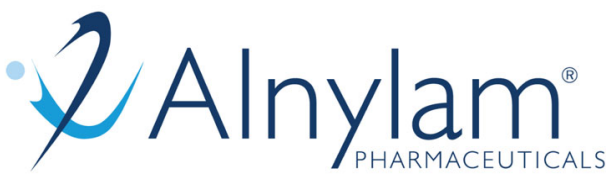




\section{Wednesday, November 6, 2019}

\section{ORAL PRESENTATIONS}

\section{Plenary Lecture 1: Robertson Plenary Lecture}

Impact of obesogenic diet on autonomic regulation in a rat model of chronic spinal cord injury

\section{G.D. Fink}

Department of Pharmacology and Toxicology, Michigan State University, East Lansing, MI, USA

The majority of individuals with spinal cord injury (SCI) are middleaged or younger so have the potential to live long and healthy lives. Nevertheless life expectancy in individuals with SCI is lower than in the able-bodied population in part because of an increased propensity for developing a variety of chronic diseases that afflict the aging population as a whole. Therefore it is important to study and understand the mechanisms by which SCI affects the development and severity of aging related chronic diseases. One such chronic condition is obesity and its associated cardiometabolic abnormalities. Weight gain has numerous adverse health effects, some of which are linked to dysregulation of the autonomic nervous system. Thus it is reasonable to expect that loss of supraspinal control of sympathetic activity in individuals with high SCI could affect the cardiometabolic sequelae of obesity. In my talk I will review and discuss preclinical experimental studies we have recently conducted with the goal of understanding the impact of an obesogenic diet in chronic SCI. Our primary experimental model was the male Dahl salt-sensitive (Dahl S) rat. We previously showed that these rats develop marked hypertension, diastolic dysfunction and renal injury when fed a high fat diet (HFD; 60\% calories from fat) from the time of weaning when compared to rats fed a control diet (CD; $10 \%$ calories from fat). Animals were subjected to T2/T3 spinal cord injury as adults and studied at least four weeks post-SCI. I will present our findings on the resulting effects on autonomic cardiovascular regulation, renal injury, and immune system function - all factors thought to contribute to accelerated mortality in individuals with SCI.

\section{Course on Autonomic Medicine}

This workshop/course introduces the broad topic of autonomic medicine, using a concept-based, didactic approach. There is an opportunity to obtain Continuing Medical Education (CME) or maintenance of certificate (MOC) credit. The course follows the general organization of Dr. Goldstein's Principles of Autonomic Medicine, which is a freely available resource that can be downloaded from his NINDS webpage https://neuroscience.nih.gov/ninds/Faculty/ Profile/david-goldstein.aspx. (The e-book is currently under revision, and suggestions for improvement are encouraged.) Topics to be covered include: Why are dysautonomias hard? What is the ANS? ANS components; organization of the ANS; chemical messengers of the ANS; homeostasis and the ANS; stress and the ANS; The Dysautonomias Universe; the syndromic nature of dysautonomias; autonomic function tests; pediatric dysautonomias; adult dysautonomias; geriatric dysautonomias; miscellaneous dysautonomias; treatment of dysautonomias (emphases on postural tachucardia syndrome and neurogenic orthostatic hypotension).

\section{Research Testing of Autonomic Function Workshop}

The autonomic nervous system is a key regulator of physiological responses required to maintain homeostatic functions including blood pressure, heart rate, respiration, and thermoregulation. Autonomic regulatory responses involve modulation of afferent neural pathways, central nervous system integration, efferent pathways controlling nerve activity to end organs, and release of hormonal factors. Since autonomic dysfunction has been implicated in numerous chronic disease states, tools to assess the status and responsiveness of the autonomic nervous system have become of critical importance. This symposium will describe recent advances in direct and indirect approaches to test autonomic function in the research setting including in vivo assessment of neurocardiovascular function in preclinical rodent models and measurement and quantitation of arterial baroreceptor reflex function, post-ganglionic sudomotor function, and sympathetic neural discharge in humans.

\section{Clinical Autonomic Testing Workshop}

Clinical autonomic testing provides an important supplementary role to the autonomic history and examination. A well-validated battery of autonomic function tests, generally referred to as Autonomic Reflex Screen (ARS), provides an evaluation of a sufficient number of autonomic systems to assess the distribution and severity of autonomic dysfunction. The ARS includes quantitative sudomotor axon reflex testing at standardized sites to assess postganglionic sympathetic sudomotor function; and heart rate and blood pressure responses to deep breathing, Valsalva maneuver, and head-up tilt to assess cardiovagal and cardiovascular adrenergic function. The tests fulfill the requirements of the currently available Current Procedural Terminology (CPT) codes on tests of autonomic function. This workshop will provide attendants with an overview of clinical autonomic testing using the ARS. It will start with a lecture to provide an overview of the physiologic basis of the ARS components, testing parameters, examples of normal and abnormal, and discussion of confounding factors. This will be followed by a live demonstration of test preparation, patient hook-up, and performing all three ARS components. The workshop will close with a presentation and discussion of interesting and illustrative cases and pitfalls of testing.

Thursday, November 7, 2019

\section{Session 1: Autonomic Regulation of Heart and Vasculature}

Evidence of adaptations in the neural control of body temperature following heat acclimation in humans

H. Barry, G. Chaseling, S. Moreault, C. Sauvageau, P. Behzadi, H. Gravel, N. Ravanelli, D. Gagnon

Department of Pharmacology and Physiology, Université de Montréal and Cardiovascular Prevention and Rehabilitation Centre, Montreal Heart Institute, Montréal, Canada

Heat acclimation enhances the heat loss responses of sweating and cutaneous vasodilation. However, it remains unknown if central adaptations in the neural control of body temperature contribute to the improvements in heat loss responses induced by acclimation. We evaluated the hypothesis that heat acclimation results in an earlier activation and greater response of skin sympathetic nerve activity (SSNA) during passive heating. Fourteen healthy adults $(27 \pm 5$ years, 9 males/5 females) underwent passive heat exposure before and after 7 consecutive days of isothermic heat acclimation (core temperature maintained at $\geq 38.6^{\circ} \mathrm{C}$ ). SSNA (microneurography at the radial nerve), local sweat rate (LSR), skin blood flow (SkBF), esophageal (Teso) and mean skin temperatures were measured throughout passive heat exposure. Heat acclimation increased whole-body sweat rate 
( $0.96 \pm 0.40$ to $1.50 \pm 0.64 \mathrm{~L} / \mathrm{h}, \mathrm{P}<0.01)$ and lowered resting Teso ( $36.85 \pm 0.27$ to $36.56 \pm 0.33^{\circ} \mathrm{C}, \mathrm{P}<0.01$ ). During passive heating, the mean body temperature onset threshold for the activation of SSNA $\left(0.98 \pm 0.23\right.$ to $\left.0.77 \pm 0.14^{\circ} \mathrm{C}, \quad \mathrm{P}<0.01\right), \quad$ LSR $\quad(1.19 \pm 0.23$ to $\left.1.06 \pm 0.22^{\circ} \mathrm{C}, \mathrm{P}=0.03\right)$ and $\operatorname{SkBF}\left(1.21 \pm 0.22\right.$ to $1.02 \pm 0.27^{\circ} \mathrm{C}$, $\mathrm{P}=0.01)$ was reduced post-acclimation. In addition, the increase in SSNA, LSR and SkBF as a function of increases in mean body temperature was greater during the post-acclimation visit (interactions, all $\mathrm{P} \leq 0.08$ ). These results suggest that adaptations in the neural control of body temperature contribute to the improvement of heat loss responses following heat acclimation in humans.

Funding: Natural Sciences and Engineering Research Council (RGPIN-2017-04243).

\section{Low-frequency sinusoidal electrical stimulation of the dorsolateral prefrontal cortex modulates muscle sympathetic nerve activity in humans}

\section{T. Dawood, L.A. Henderson, V.G. Macefield}

Human Autonomic Neurophysiology Lab, Baker Heart and Diabetes Institute, Melbourne, Australia

Using concurrent microneurography and functional magnetic resonance imaging of the brain we have previously documented that activity in the dorsolateral prefrontal cortex (dIPFC) is functionally coupled to the generation of muscle sympathetic nerve activity (MSNA) in young healthy individuals (James C, Macefield VG \& Henderson LA, NeuroImage 2013;70:59-65). Given that the dIPFC is a common target for modulation, through the use of transcranial direct current stimulation (tcDCS), we reasoned that tcDCS should be able to modulate MSNA in the same way that sinusoidal galvanic stimulation modulates (sGVS) MSNA (Hammam E, James C, Dawood T \& Macefield VG, Exp Brain Res 2011;213:507-514). Multi-unit recordings of MSNA were obtained from the peroneal nerve in 6 female and 3 male participants (age 23-35 y). Surface $\mathrm{Ag}-\mathrm{AgCl}$ electrodes were placed over the left and right dlPFC, corresponding to EEG electrode positions F3 and F4, respectively, and over the nasion. Unilateral, low-frequency sinusoidal stimulation $( \pm 2 \mathrm{~mA} @ 0.08 \mathrm{~Hz})$ was applied between F3 or F4 and the nasion for 100 cycles each. Negative-going sympathetic spikes were extracted from the nerve signal and cross-correlation histograms constructed between the sympathetic spikes and the sinusoidal stimulus. In all participants there was a significant cyclic modulation of MSNA $(\mathrm{P}<0.001)$. The mean $( \pm \mathrm{SD})$ modulation index was $16.9 \pm 8.0 \%$ and $16.4 \pm 4.7 \%$ for the contralateral and ipsilateral stimulation, respectively. We conclude that sinusoidal electrical stimulation of the dIPFC modulates MSNA, presumably through projections between the dIPFC and the rostral ventrolateral medulla, the final output nucleus in the brainstem for MSNA (James et al., 2013), further supporting a role for higher brain areas in the control of blood pressure.

Funding: National Health and Medical Research Council GTN1100042.

\section{Sympathetic vasodilation improves insulin-mediated microvascular recruitment in the forearm of obese human subjects}

J. Celedonio, J.N. Patel, A. Ruiz, E.C. Smith, S. Lonce, S. Paranjape, L. Okamoto, I. Biaggioni, A. Gamboa

Vanderbilt University Medical Center, Nashville, TN, USA

Background: Obesity is associated with sympathetic activation, endothelial dysfunction, insulin resistance and blunted insulin-mediated microvascular recruitment. We have previously shown that acute pharmacological blockade of the autonomic nervous system improves both insulin resistance and endothelial dysfunction. We now propose that removal of sympathetic vasoconstriction can result in improvement of insulin-mediated vasodilation and subsequently in sensitivity to insulin-mediated glucose uptake.

Methods: We blocked sympathetic vasoconstriction in an isolated forearm model during a hyperinsulinemic euglycemic clamp to determine the effect of insulin recruitment on microvascular circulation using contrast-enhanced ultrasonography (CEU). We studied 9 obese human subjects (age $41 \pm 6$ years, BMI $37 \pm 3 \mathrm{~kg} / \mathrm{m}^{2}$, SBP $125 \pm 6 \mathrm{~mm} \mathrm{Hg}$ ). We assessed the effects of insulin on microvascular recruitment and glucose uptake on two separate occasions, randomly assigned and at least one month apart, during an intrabrachial infusion of the alpha-adrenergic blocker phentolamine (PHE, 25 micrograms/ min, blocked day) or saline (SAL, control day). Subjects were studied at baseline (BSL) and at the end of the clamp (CLAMP). The main outcome chosen was peak microvascular blood volume (MBV), an index of microvascular recruitment measured as intensity units (IU). Results: As expected, forearm blood flow increased after insulin alone $(67 \pm 27 \%, p=0.016)$ and with alpha blockade and insulin $(140 \pm 22 \%$, $\mathrm{p}=0.0011)$. MBV showed a significant increase during PHE compared to SAL (from $6.4 \pm 3.2 \mathrm{IU}$ to $8.7 \pm 3.4 \mathrm{IU}, \mathrm{p}=0.0978$ for $\mathrm{SAL}$, and from $6.7 \pm 1.0 \mathrm{IU}$ to $11.9 \pm 2.7 \mathrm{IU}, \mathrm{p}=0.0467$ for PHE). Muscle glucose uptake trended towards improvement with PHE but did not reach statistical significance $(\mathrm{p}=0.0558)$. There were no systemic effects due to brachial sympathetic blockade.

Conclusions: This data shows that sympathetic vasoconstriction contributes to the blunted insulin-mediated microvascular recruitment seen in obesity. This study suggests that targeting sympathetic overactivity in obese subjects can be used to treat the associated complications of obesity.

\section{Differences in regional grey matter volume of the brain are related to mean blood pressure and muscle sympathetic nerve activity in humans}

S. Kobuch, R.H. Fatouleh, J.M. Macefield, L.A. Henderson, V.G. Macefield

Human Autonomic Neurophysiology Lab, Baker Heart and Diabetes Institute, Melbourne, Vic, Australia

The brainstem plays a critically important role in the beat-to-beat control of blood pressure, as well as setting mean blood pressure (MBP). We recently showed that regional cerebral blood flow to specific brainstem nuclei is inversely related to resting MBP in healthy normotensive individuals. Here we tested the hypothesis that grey matter volume in these same nuclei, and areas above the brainstem to which they are connected, is also associated with resting MBP and muscle sympathetic nerve activity (MSNA). Structural magnetic resonance imaging (MRI) of the brain and recordings of MSNA and BP were collected in 54 healthy participants. Subjects were divided into a lower MBP group (mean \pm SEM $78.8 \pm 1.5 \mathrm{mmHg}$, $\mathrm{n}=27)$ and higher MBP group $(96.6 \pm 1.2 \mathrm{mmHg}, \mathrm{n}=27)$, as well as into a lower MSNA ( $9.5 \pm 0.8$ bursts/min, $\mathrm{n}=27)$ and higher MSNA $(25.4 \pm 1.2$ bursts/min, $n=27)$ group. Regional grey matter volume was higher in the region of the rostral ventrolateral medulla, nucleus tractus solitarius, and medullary raphe in the group with higher MBP and correlated significantly with mean MBP across all subjects. Grey matter volume was significantly higher in the dorsomedial hypothalamus and anterior and posterior cingulate cortices in the group with lower MSNA and was inversely related to MSNA across all subjects. We conclude that small differences in MBP and MSNA are associated with significant differences in grey matter volume in cortical and subcortical regions known to be involved in blood pressure regulation, suggesting that these structural differences contribute to resting MBP and MSNA and can predict the establishment of hypertension. 
Funding: National Health and Medical Research Council GTN1100042.

\section{Impact of age and sex on neural cardiovascular responsiveness to cold pressor test in humans}

\author{
M.L. Keller-Ross ${ }^{1}$, H.A. Cunningham ${ }^{2}$, J.R. Carter ${ }^{2}$ \\ ${ }^{1}$ Department of Rehabilitation Medicine, School of Medicine, \\ University of Minnesota, Minneapolis, MN, USA; ${ }^{2}$ Department of \\ Kinesiology \& Integrative Physiology, Michigan Technological \\ University, Houghton, MI, USA
}

Age and sex are known factors that influence hypertension risk, with postmenopausal women being at greatest risk. Prior longitudinal work suggests that blood pressure reactivity to the cold pressor test (CPT) helps predict hypertension, yet the impact of age and sex on hemodynamic and neural responsiveness to CPT remain equivocal. Fortythree young $(21 \pm 1 \mathrm{yrs}$, mean $\pm \mathrm{SEM})$ men $(\mathrm{YM}, \mathrm{n}=20)$ and women (YW, $\mathrm{n}=23)$, and 19 older ( $60 \pm 1 \mathrm{yrs})$ men $(\mathrm{OM}, \mathrm{n}=10)$ and women $(\mathrm{OW}, \mathrm{n}=9)$ participated in an experimental visit where continuous blood pressure (BP; finger plethysmography) and muscle sympathetic nerve activity (MSNA; microneurography) were measured during a 3-5 min baseline and 2-min CPT of the hand. Body mass index was higher in older compared with younger adults $\left(27 \pm 1 \mathrm{vs.} 24 \pm 0 \mathrm{~kg} / \mathrm{m}^{2}\right.$, $\mathrm{p}=0.05$ ). Baseline mean arterial pressure (MAP) was greater in older compared with younger adults, $(88 \pm 3$ vs. $75 \pm 1 \mathrm{mmHg}, \mathrm{p}=0.05)$. Baseline MSNA was greater in OM (69 \pm 6 bursts/100 hb) compared with OW ( $48 \pm 6$ bursts $/ 100 \mathrm{hb}, \mathrm{p}=0.02)$, and lower in young adults (YM: $17 \pm 3$ vs. YW: $16 \pm 2$ bursts/100 hb, $\mathrm{p}<0.01$ ), but similar across sexes $(\mathrm{p}=0.83)$. However, when exposed to the CPT, MSNA increased more in OW $(\Delta 28 \pm 6$ bursts $/ 100 \mathrm{hb}$; time $\times$ age $\times$ sex interaction, $\mathrm{p}=0.01)$ compared with $\mathrm{OM}(\Delta 7 \pm 6$ bursts/100 hb), but was not different between YW $(\Delta 25 \pm 4$ bursts/100 hb) and YM $(\Delta 24 \pm 4$ bursts/100 hb). Despite the augmented MSNA reactivity in OW, OW and OM had similar increases in MAP $(\Delta 27 \pm 6$ vs. $\Delta 26 \pm 6 \mathrm{mmHg}$, $\mathrm{p}=0.86$ ), which was greater than that observed in young adults (time $\times$ age interaction, $\mathrm{p}=0.008$ ). In conclusion, these findings suggest that while MSNA and BP responsiveness to CPT are similar between $\mathrm{YM}$ and $\mathrm{YW}$, there is a divergence with age in which there is greater sympathoexcitation to CPT in OW compared to OM, despite similar $\mathrm{BP}$ responsiveness. This greater sympathetic reactivity in OW may be a contributing mechanism to the increased hypertension risk in postmenopausal women.

Funding: National Institutes of Health HL122919, HL088689, HL098676 and the Portage Health Foundation.

\section{Neuroplasticity in nucleus tractus solitarii after cardiovascular deconditioning induced by hindlimb unloading in rats}

\section{Lima-Silveira, E.M. Hasser, D.D. Kline \\ Department of Biomedical Sciences and Dalton Cardiovascular \\ Research Center, University of Missouri, Columbia, MO, USA}

The nucleus tractus solitarii (nTS) is the first brainstem region to receive cardiovascular afferent information. Alterations in glutamatergic synaptic transmission in the nTS contributes to several cardiovascular dysfunctions. In this study, we investigated the effects of hindlimb unloading (HU), a well-established model of cardiovascular deconditioning in rats, on glutamatergic synaptic transmission and intrinsic properties of nTS neurons. For this purpose, stainless steel wire rings were placed in the tail of male Sprague-Dawley rats (3 weeks) and after one week of recovery the rings were connected to a suspension apparatus. HU rats were suspended at an angle of 30-35 for two weeks with free access to food and water, while control animals were maintained under normal postural conditions. After this period, coronal brainstem slices were generated and the whole-cell patch clamp technique was used to evaluate the electrophysiological properties of nTS neurons. The effectiveness of the HU protocol was confirmed by significant hindlimb muscle atrophy in comparison to control animals. Adrenal weight was comparable between HU and control rats. HU increased the amplitude of excitatory postsynaptic currents evoked by afferent tractus solitarii stimulus (TS-EPSC), although it does not affect the frequency and amplitude of spontaneous network EPSCs. Neurons of HU rats were hyperpolarized, which is consistent with the reduced percentage of neurons that exhibited spontaneous firing activity (Control: $54 \%$ vs. HU: $13 \%$ ). Additionally, $\mathrm{HU}$ increased action potential (AP) rheobase and decreased the number of AP generated with step-depolarization. Taken together, these data indicate that HU increases glutamatergic neurotransmission and reduces the excitability of nTS neurons. These results suggest that functional neuroplasticity in the nTS may contribute to the cardiovascular deconditioning following $\mathrm{HU}$ in rats.

Funding: Support: HL132836 and AAS-Lundbeck Research Fellowship Grant.

\section{Symposium 1: Role of Autoimmunity in POTS}

Postural tachycardia syndrome (POTS) is a clinically defined syndrome that is not a homogenous disorder, but rather a set of symptoms that represents the outward manifestation of diverse underlying processes. In many patients with POTS, there is evidence of dysregulation of the immune system. Various serum autoantibodies directed against targets in the autonomic system have been reported in POTS patients but the significance of these antibodies is still uncertain. In some cases, an associated defined autoimmune disorder can be identified which appears to be the main cause of the symptom complex. In most cases however, the evidence for an autoimmune cause for POTS is indirect. Immunomodulatory treatment has been tried in some patients but no controlled clinical trials have been conducted. There is a strong link between the immune system and the autonomic nervous system, and further research into this link promises to yield new insights and novel treatment approaches for POTS and other forms of dysautonomia.

\section{Session 2: Autonomic Neuropathy and POTS}

Low frequency variability of systolic arterial pressure accurately predicts autonomic injury during the acute phase of spinal cord injury

V.E.M. Lucci, J.A. Inskip, M.S. McGrath, B.K. Kwon, V.E. Claydon Department of Biomedical Physiology and Kinesiology, Simon Fraser University, Burnaby, BC, Canada

Introduction: Spinal cord injury (SCI), particularly high-level (T6 or above) SCI, injures cardiovascular autonomic (sympathetic) nerves and can lead to profound cardiovascular dysfunction. However, there is currently no gold-standard for quantitatively assessing autonomic function following SCI. Additionally, the evolution of cardiovascular autonomic function in the acute phase (first year) following injury is unknown. We evaluated cardiovascular autonomic function during acute SCI, with the intent of distinguishing individuals with autonomically-complete and autonomically-incomplete injuries.

Methods: We tested 60 individuals with acute traumatic SCI (age at injury: $47.1 \pm 15.7$ years) at four timepoints: $<2$ weeks, 1-, 3-, and 6-months post injury. Supine beat-to-beat blood pressure and heart rate were recorded. At each visit, low frequency variability in systolic arterial pressure (LFSAP) was determined. As LFSAP is a direct 
measure of sympathetic modulation of blood pressure, we used this as our primary outcome measure, with blunted/absent LFSAP suggesting autonomically-complete injuries.

Results: Most lesions (72\%) were T6 or above, putting individuals at risk for cardiovascular dysfunction; $35 \%$ had motor/sensory complete lesions. LFSAP was not related to conventional measures of motor/ sensory impairment such as level of injury $(\mathrm{p}=0.8)$ or motor and sensory grade $(\mathrm{p}=0.2)$. Regardless of lesion level, LFSAP was decreased at $<2$ weeks $\left(3.3 \pm 1.3 \mathrm{mmHg}^{2}\right)$, highlighting the impact of neurogenic shock/acute trauma on autonomic control. Based on this early disruption in autonomic function, autonomic injury was then defined as a blunted LFSAP $\left(<3.5 \mathrm{mmHg}^{2}\right)$ in participants with lesions T6 or above. Using these criteria, two distinct groups emerged by 1-month: autonomically-complete SCI with sustained low LFSAP $\left(1.4 \pm 0.3 \mathrm{mmHg}^{2}\right)$ and autonomically-incomplete SCI with increased LFSAP $\left(8.9 \pm 2.6 \mathrm{mmHg}^{2}, \mathrm{p}<0.001\right)$. This relationship persisted at subsequent timepoints $(\mathrm{p}<0.001)$. There was no evidence of autonomic recovery over time in autonomically-complete injuries.

Conclusion: Use of LFSAP provides a simple, non-invasive assessment of autonomic function, allowing for the first-ever quantitative evaluation of autonomic function following SCI, and is most informative 1-month after injury.

Funding: Heart and Stroke Foundation of British Columbia and Yukon.

\section{Clinical phenotyping of postural tachycardia syndrome}

B. Roschach, V. Galvis, A.I. Penzlin, I. Bonyhay, J. Celli, R. Freeman, C.H. Gibbons

Department of Neurology, Beth Israel Deaconess Medical Center, Harvard Medical School, Boston, MA, USA

Objective: To generate a data-driven phenotyping of postural tachycardia syndrome (POTS) through cluster analysis of autonomic function, medical history and response to symptom questionnaires.

Background: POTS is a heterogeneous disorder of orthostatic intolerance. Current phenotyping models have not successfully translated into pathophysiology-based treatments.

Methods: We reviewed the medical records and autonomic tests of all patients diagnosed with POTS at our institution from 2008-2019. Autonomic testing included a 45 minute tilt-table test, Valsalva maneuver (VM) and evaluation of heart rate variability (HRV). All patients completed the Boston Autonomic Questionnaire. We performed a provisional principal component analysis (PCA).

Results: PCA of the first 50 patients revealed peripheral vasomotor discoloration as a distinct cluster. 24/50 (48\%) reported discoloration, with $9(18 \%)$ describing an isolated red discoloration and 15 $(30 \%)$ reporting a blue-mixed pattern. Patients with discoloration exhibited a higher maximum heart rate on tilt (123 vs. $109 \mathrm{bpm}$, $\mathrm{p}<0.05$ ) and decreased late phase 2 blood pressure (BP) during VM (101 vs. $117 \mathrm{mmHg}, \mathrm{p}<0.01)$. These patients more frequently reported gastrointestinal symptoms including constipation (83\% vs. $46 \%, \mathrm{p}<0.01)$ and decreased appetite $(96 \%$ vs. $46 \%, \mathrm{p}<0.001)$, and reported more difficulties seeing in bright light ( $79 \%$ vs. $46 \%$, $\mathrm{p}<0.05)$ and $\operatorname{dim}$ light $(67 \%$ vs. $23 \%, \mathrm{p}<0.005)$. Patients reporting red discoloration displayed lower resting systolic BP (111.43 vs. $117.4 \mathrm{mmHg}, \mathrm{p}<0.05$ ), while those describing blue discoloration exhibited higher resting diastolic BP $(71$ vs. $62 \mathrm{mmHg}, \mathrm{p}<0.05)$, decreased Valsalva ratio $(1.89$ vs. $2.31, \mathrm{p}<0.05)$ and decreased HRV (28.1 vs. $38.6 \mathrm{bpm}, \mathrm{p}<0.05$ ). Anhidrosis was reported more frequently with blue discoloration $(47 \%$ vs. $0 \%, \mathrm{p}<0.05)$, while patients with red discoloration expressed increased urinary hesitancy (67\% vs. $20 \%, \mathrm{p}<0.05)$.
Discussion: Vasomotor discoloration may represent a novel discriminating feature of a POTS phenotype. Further investigation is required to determine whether discoloration reflects sub-phenotypes or divergent disease mechanisms and the implications for treatment.

\section{Orthostatic intolerance in the young and the benefits of oral rehydration}

M.S. Medow, K. Guber, S. Chokshi, C. Terilli, P. Visintainer, J.M. Stewart

Department of Pediatrics, New York Medical College, Valhalla, NY, USA

Background: The imposition of an orthostatic stress (standing up) causes rapid gravitational displacement of $500-700 \mathrm{ml}$ from central to splanchnic and lower extremity vascular beds. Uncompensated displacement can result in orthostatic intolerance (OI). A common cause of OI in younger patients is postural tachycardia syndrome (POTS). Intravenous central blood volume expansion with isotonic saline is commonly and effectively used to reduce OI but has long term complications. An isotonic oral rehydration solution (ORS W.H.O. formula) efficiently rehydrates cholera patients, suggesting an ability to increase central BV rivaling intravenous fluids.

Methods: We studied the neurovascular response to fluid loading during orthostatic stress using lower body negative pressure (LBNP) in 10 POTS subjects with OI and 15 controls, and on subsequent days before, and 1 hour after intravenous saline or ingestion of ORS.

Results: POTS subjects exhibited reduced tolerance $(\mathrm{p}<0.0001)$ to LBNP compared to controls (Orthostatic Index of 35,715 $\pm 3,469$ vs. $93,980 \pm 7,977$, respectively). In POTS, following ORS but not saline, cerebral blood flow velocity $(\mathrm{CBFv})$ was significantly higher than no treatment $(\mathrm{p}<0.0005)$ at $-45 \mathrm{mmHg}$. While fluid loading did not confer any advantage in Controls, POTS subjects experienced a significant improvement in orthostatic tolerance following both saline $(100 \pm 9.7$ vs. $134.5 \pm 17.4, \mathrm{p}<0.05)$ and ORS $(100 \pm 9.7$ vs. $155.6 \pm 15.7, \mathrm{p}<0.001)$ when evaluated by normalized orthostatic index ( $<<0.001$, compared to untreated baseline).

Conclusion: Maintenance of CBFv may have resulted in improved short-term orthostatic tolerance exhibited by POTS subjects following ORS administration. ORS is a convenient, safe and effective therapy for short-term relief of OI.

Funding: R21 NS 094644 from the National Institute of Neurologic Disorders and Stroke, and R01 HL 112736 and R01 HL 134674 from the National Heart Lung and Blood Institute. Funded by the National Institutes of Health.

\section{Hereditary transthyretin (hATTR) amyloidosis: symptoms and signs in early disease}

C.H. Gibbons ${ }^{1}$, A. Gonzalez-Duarte ${ }^{2}$, F. Barroso ${ }^{3}$, M. Campagnono ${ }^{1}$, J. Garcia ${ }^{1}$, S. Rajan ${ }^{1}$, R. Freeman ${ }^{1}$

${ }^{1}$ Department of Neurology, Beth Israel Deaconess Medical Center, Harvard Medical School, Boston, MA, USA; ${ }^{2}$ Instituto Nacional de Ciencias Medicas y Nutricion Salvador Zubiran, Mexico City, Mexico; ${ }^{3}$ Institute for Neurologic Research Raúl Carrea, FLENI, Buenos Aires, Argentina

Introduction: hATTR amyloidosis is due to one of many mutations in the transthyretin (TTR) gene, resulting in a progressive fatal disease with sensory, motor and autonomic involvement.

Objective: To characterize the symptoms, signs and skin biopsy neuropathological findings in a cohort of individuals with TTR mutations. 
Methods: Individuals with a variety of TTR mutations underwent detailed neurological examinations including the Neuropathy Impairment Score in the Lower Limb (NIS-LL), the Utah Early Neuropathy Score (UENS), Coutinho staging, autonomic testing, symptom scores (using the EuroQol, Brief Pain Symptom Inventory, and the Orthostatic Hypotension Questionnaire). All subjects had 3 $\mathrm{mm}$ punch skin biopsies at the distal leg and distal thigh with analysis of amyloid burden by Congo Red, and neuropathy severity by staining with protein gene product 9.5 .

Results: A total of 88 subjects with TTR mutations were included: 47 Coutinho stage $0 ; 32$ Coutinho stage 1; 9 Coutinho stages 2-3. Of those who were Coutinho stage $0,81 \%$ had amyloid detected on punch skin biopsy. The presence of amyloid was associated with greater orthostatic and vasomotor symptoms $(\mathrm{P}<0.05)$, greater pain $(\mathrm{P}<0.01)$, greater reported symptoms of neuropathy $(\mathrm{P}<0.01)$, greater dysfunction on the Norfolk Quality of life scale $(\mathrm{P}<0.01)$. Similarly, in individuals with NIS-LL (neuropathy) scores of 0 , the presence of amyloid on skin biopsy was associated with greater symptoms of pain, disability, orthostatic and vasomotor symptoms $(\mathrm{P}<0.05)$.

Conclusion: Skin biopsy is a sensitive pathological marker for tissue diagnosis of TTR-FAP across multiple mutations, even in individuals with no clinical evidence of disease as defined by normal examination scores. These data support the use of skin biopsy derived measurement of IENFD and amyloid deposition as a diagnostic biomarker for the peripheral neuropathy associated with hATTR. The linear relationship between disease progression and these measures support the use of the amyloid deposition index as a therapeutic biomarker. Funding: Funded by Pfizer (RF).

\section{Putative genetic markers for postural tachycardia syndrome}

J.R. Boris, H. Hakonarson

Children's Hospital of Philadelphia, Philadelphia, PA, USA

Background: The pathophysiology of postural tachycardia syndrome (POTS) has been described as being a failure of ability to maintain vascular tone with a compensatory tachycardia. There is associated chronic orthostatic intolerance and severe debilitating symptoms. However, the actual genetic mechanism of POTS is not understood. We performed a genome wide association analysis (GWAS) to determine the genetic underpinnings of POTS.

Methods: We recruited 465 patients diagnosed with POTS and available family members. We obtained a biological sample (blood or saliva) for DNA isolation and GWAS analysis using the Illumina Infinium Global Screening Array. We performed transmission disequilibrium testing (TDT) in available triads. With Bonferroni correction, significance was defined as 7.14E-08.

Results: TDT analysis was performed in 102 family triads, and case control analysis in 300 patients versus 4,328 ethnicity-matched controls. A non-coding variant in the protein phosphorylase 1 regulatory subunit 12B (PPP1R12B) for myosin, located on chromosome 1q32.1, demonstrated significance at $2.35 \mathrm{E}-08$. This was replicated in both independent TDT and case-control analysis. Also, a non-coding variant of human zinc ribbon domain antisense RNA 1 (ZNRD1AS1), in the major histocompatibility class (MHC) I region located on chromosome 6, demonstrated suggested significance at 5.22E-07.

Discussion: PPP1R12B, which dephosphorylates the regulatory light chain of myosin II, is a candidate marker for POTS. This could be a clinically plausible gene for POTS, as myosin is expressed in multiple locations in the body, including vascular smooth muscle. ZNRD1AS1 is a long non-protein coding RNA. Many patients have onset of symptoms after an infection or concussion, suggesting an autoimmune etiology involving the MHC region. While these specific loci are considered to be non-coding in humans, these variants could interfere with RNA expression of other genes. Further exome and
RNA sequencing is underway to delineate these loci to help determine further clinical validity.

Funding: Esther Feigenbaum Family Foundation.

Responsiveness of neuropathy symptom and change (NSC) score components in inotersen treatment of hereditary transthyretin amyloidosis polyneuropathy

P.J.B. Dyck, T. Coelho, M. Waddington Cruz, T. Brannagan, S. Khella, C. Karam, J.L. Berk, M.J. Polydefkis, J.C. Kincaid, J.F. Wiesman, W.J. Litchy, M.L. Mauermann, E.J. Ackermann, B.F. Baker, S.W. Jung, S.Guthrie, M. Pollock, P.J. Dyck Mayo Clinic, Rochester, MN, USA

Background: Hereditary transthyretin-mediated amyloidosis (hATTR) is a rare, progressive, fatal disease in which buildup of transthyretin protein in major organ systems results in organ failure. The transthyretin-directed antisense oligonucleotide, inotersen, is indicated for treatment of the polyneuropathy of hATTR amyloidosis and demonstrated significant benefit in the global, double-blind, placebo-controlled phase 3 study (NEURO-TTR, NCT01737398) by the coprimary end points: modified Neuropathy Impairment Score +7 physiologic tests (mNIS+7) and Norfolk Quality of Life-Diabetic Neuropathy questionnaire (Norfolk QoL-DN). Reported here are the effects of inotersen on the neuropathy symptoms and change (NSC) score, an exploratory end point in the NEURO-TTR study. The NSC questionnaire comprises 38 questions on polyneuropathy symptoms, 9 of which relate to autonomic symptoms.

Methods: Adults with neuropathy stage 1 or 2 hATTR amyloidosis were randomly assigned $(2: 1)$ to $300 \mathrm{mg}$ weekly subcutaneous doses of inotersen or placebo for 15 months. The NSC score (total and subdomains) was assessed in tandem with the primary end points at 8 and 15 months.

Results: Of 165 patients, inotersen-treated patients reported significant improvement versus placebo in NSC total score from baseline to 8 months (least-squares mean [LSM] difference, $-3.28 ; 95 \% \mathrm{CI}$, -5.70 to $-0.86 ; \mathrm{P}=0.008$ ) and 15 months (LSM difference, -6.33 ; $95 \% \mathrm{CI},-9.12$ to $-3.55 ; \mathrm{P}<0.001)$. Inotersen-treated patients also experienced relative improvements at 15 months in subdomains of muscle weakness $(-3.07 ; \mathrm{P}<0.001)$, sensory $(-1.9 ; \mathrm{P}=0.005)$, pain $(-1.59 ; \mathrm{P}<0.001)$, and autonomic symptoms $(-1.36 ; \mathrm{P}=0.008)$ when compared with baseline status.

Conclusion: Patients receiving inotersen experienced therapeutic benefit versus placebo in total NSC score and in subdomains of muscle weakness, sensory, pain, and autonomic symptoms at 15 months. The benefits of inotersen on neuropathic symptoms in patients with hATTR amyloidosis correlate with previously reported effects on neuropathy (mNIS +7 ) and quality of life (Norfolk QOLDN).

Funding: This study was sponsored by Ionis Pharmaceuticals, Inc. Akcea Therapeutics is an affiliate of Ionis Pharmaceuticals, Inc.

\section{Symposium 2: Sex Differences in Autonomic Control of Blood Pressure and Metabolism}

Cardiovascular disease is the leading cause of death for women in the United States. Minority groups such as African American woman are particularly impacted by cardiovascular disease. For the past decades, dramatic declines in cardiovascular mortality have been observed in the $>65$-year age group. However, recent data suggest stagnation in the morbidity and mortality of coronary artery disease among young women ( $<55$ years). These observations could be explained in part by the differential impact of traditional risk factors on women as well as the presence of non-traditional risk factors. In the present symposium, 
we will discuss novel findings on sex-difference as a biological variable in the mechanisms underlying cardiovascular disease, hypertension and metabolic syndrome. We will provide special emphasis on novel RAS pathways and metabolic control, the effect of pregnancy in autonomic mechanisms of hypertension, and cholinergic regulation of oxidative stress in African American women. The overall goal of this symposium is to provide cutting edge science on the role of sex difference on the cardiovascular conditions that mostly affect women.

\section{Angiotensin-(1-7) and metabolic function in obesity: sex matters}

\section{A.C. Arnold}

Department of Neural and Behavioral Sciences, Penn State College of Medicine, Hershey, PA, USA

Obesity is a global epidemic that greatly increases the risk for developing cardiovascular disease and type II diabetes. Sex differences in the obese phenotype are well established in preclinical models and clinical populations, with females generally exhibiting protection from obesity-induced metabolic and cardiovascular complications despite increased adiposity. This protection is attributed, in part, to sex differences in hormonal mediators such as the reninangiotensin system (RAS). Our emerging data provides evidence for sex differences in the role of angiotensin (Ang)-(1-7), a beneficial hormone of the RAS, in metabolic protection in obesity. Previous studies from our group and others have shown that circulating levels of Ang-(1-7) are greatly reduced in obese male mice, and that restoration of this hormone is protective for blood pressure, glucose homeostasis, and energy balance in this model. This lecture will describe findings in obese male mice as well as present more recent data showing female mice are more responsive to chronic Ang-(1-7) treatment in terms of energy balance and glucose homeostasis, and potential autonomic mechanisms involved. These overall findings improve understanding of sex differences in RAS mechanisms in obesity and may inform on the potential for targeting Ang-(1-7) for treatment of obesity and related metabolic and cardiovascular risk.

\section{Neural control of blood pressure during pregnancy in humans}

\section{Q. Fu}

Institute for Exercise and Environmental Medicine at Texas Health Presbyterian Hospital Dallas; The University of Texas Southwestern Medical Center, Dallas, TX, USA

Pregnancy is associated with significant changes in maternal hemodynamics, which begin as early as 4 weeks of gestation and reach a plateau in the second trimester. It has been proposed that these changes occur through autonomic control mechanisms, but the actual role of the sympathetic nervous system in pregnancy remains largely unclear. Previous microneurographic studies found that muscle sympathetic nerve activity (MSNA) increased in normotensive pregnant women and was even greater in women with gestational hypertension and preeclampsia during the third trimester. It is possible that sympathetic activation during the latter months of normal pregnancy helps return the arterial pressure to non-pregnant levels, however, when the increase in sympathetic activity is excessive, hypertension ensues. The key question that must be addressed is whether sympathetic activation develops early during pregnancy and remains high throughout gestation, or whether this sympathetic overactivity only occurs at term, providing the substrate for preeclampsia and other pregnancy associated cardiovascular complications. We found that resting MSNA increased in early-pregnancy, further increased in late-pregnancy, and returned to the pre-pregnancy level shortly after delivery in healthy women. Recent work from our laboratory showed early signs of excessive sympathetic activation (during the first trimester, before any clinical signs and symptoms appear) in women who developed gestational hypertension at term. These findings provide important insight into the neural mechanisms underlying hypertensive disorders during pregnancy. We also found that corin, an atrial natriuretic peptide-converting enzyme, was increased in the maternal circulation as a homeostatic response to elevated sympathetic activity. Our study suggests that corin plays a unique role in blood pressure regulation throughout normotensive and, especially hypertensive pregnancy and may represent a promising biomarker for determining women at high risk of adverse pregnancy outcomes. With this knowledge, early prevention or treatment targeted to the appropriate pathophysiology may be initiated, which may reduce maternal and fetal death or morbidity, as well as cardiovascular risks in women later in life.

\section{Racial difference in cholinergic modulation of oxidative stress and its clinical application in obese women}

\section{C.A. Shibao}

Vanderbilt University, Nashville, TN, USA

African Americans account for $\sim 13 \%$ of the US population and represent the second largest racial minority in the US. Despite the advances in the identification of risk factors and the use of evidencebased strategies to treat cardiovascular diseases (CVD), the mortality rate remains higher in $\mathrm{AA}$ than whites. The role of the autonomic nervous system in the regulation of hypertension and metabolism has been actively studied. A major focus of this session is to highlight the cholinergic signaling and its contribution to inflammation and oxidative stress in African Americans. This knowledge paved the way to recent clinical trials with cholinergic drug - galantamine and its role in lipid-induced oxidation in this population.

\section{Compensatory interaction between sympathetic baroreflex} sensitivity and vascular transduction in males but not females

C.E. Taylor ${ }^{1,2}$, S.L. Hissen ${ }^{1}$, R. Brown ${ }^{2,3}$, V.G. Macefield ${ }^{2,3,4}$

${ }^{1}$ School of Science and Health, Western Sydney University, Sydney, Australia; ${ }^{2}$ School of Medicine, Western Sydney University, Sydney, Australia; ${ }^{3}$ Neuroscience Research Australia, Sydney, Australia;

${ }^{4}$ Baker Heart and Diabetes Institute, Melbourne, Australia

Sympathetic baroreflex sensitivity (BRS) is a measure of how effectively the baroreflex buffers beat-to-beat changes in blood pressure through the modulation of muscle sympathetic nerve activity (MSNA). However, current methods of assessment do not take into account the transduction of MSNA at the level of the vasculature, which is known to vary between individuals. In this study we test the hypothesis that there is an inverse relationship between sympathetic BRS and vascular transduction, and thus a compensatory interaction between these two components of baroreflex function. A secondary aim was to examine the influence of resting MSNA on sympathetic BRS and vascular transduction. In 38 (18 males) healthy adults, continuous measurements of blood pressure, MSNA (microneurography) and superficial femoral artery diameter and blood flow (Doppler ultrasound) were recorded during 10 min of rest. Spontaneous sympathetic BRS was quantified as the relationship between diastolic pressure and MSNA burst incidence (Ensemble R). Vascular transduction was quantified by plotting the changes in vascular conductance for 10 cardiac cycles following each burst of MSNA, and taking the nadir. In males, sympathetic BRS was inversely related to vascular transduction $(r=0.49 ; \mathrm{p}=0.04)$. However, this relationship 
was not present in females $(\mathrm{r}=0.17 ; \mathrm{p}=0.47$ ). Resting MSNA was positively related to sympathetic BRS and vascular transduction in males but not females. To conclude, compensatory interactions exist between sympathetic BRS and vascular transduction in healthy young males, which may serve to ensure homeostasis. Males with attenuated vascular transduction compensate with greater levels of resting MSNA and greater changes in MSNA in response to changes in pressure (sympathetic BRS). Further research is required to determine whether these compensatory interactions respond dynamically under physiological stress and why they are not apparent in young females. Funding: National Health \& Medical Research Council of Australia (GNT1029782 and GNT11000420).

Sympathetic and cardiovascular responses to acute mental stress in post-traumatic stress disorder (PTSD): sex differences

\section{I.T. Fonkoue, T.N. Jones, M. Vemulapalli, D. DaCosta, M.L.} Kankam, J. Park

Renal Division, Department of Medicine, Emory University School of Medicine, Atlanta, GA; Research Service Line, Atlanta VA Medical Center, Decatur, GA

Post-traumatic stress disorder (PTSD) is associated with significantly higher risk of developing hypertension. Women are twice as likely as men to develop PTSD after a traumatic event. Our prior studies showed that sympathetic nervous system (SNS) reactivity during combat and non-combat related stress is heightened in PTSD; however, these prior results were obtained in predominantly male cohort and did not consider the potential influence of sex on neural cardiovascular function in PTSD. The aim of the current study was to determine if women with PTSD have heightened heart rate (HR), blood pressure (BP) and SNS reactivity to acute mental stress than men with PTSD. To answer this question, we recruited 25 veterans (17 men and 8 women) clinically diagnosed with PTSD using the Clinician Administered PTSD scale (CAPS). Electrocardiography (EKG), seated and beat-to-beat supine BP and muscle sympathetic nerve activity (MSNA) were measured at baseline and during $3 \mathrm{~min}$ of mental stress via mental arithmetic. Men and women were matched for age ( $35 \pm 2$ vs. $38 \pm 2$ years) and body mass index (BMI) (29 \pm 2 vs. $\left.31 \pm 3 \mathrm{~kg} / \mathrm{m}^{2}\right)$. Baseline HR ( $70 \pm 3$ vs. $73 \pm 4$ beats $\left./ \mathrm{min}\right)$, seated systolic BP (122 \pm 3 vs. $121 \pm 6 \mathrm{mmHg})$, seated diastolic BP (77 \pm 3 vs. $79 \pm 4 \mathrm{mmHg}$ ) and MSNA ( $24 \pm 3$ vs. $29 \pm 5 \mathrm{mmHg}$ ) were comparable between men and women. In response to mental arithmetic, there was a greater systolic BP response ( $124 \pm 6$ to $134 \pm 6$ vs. $125 \pm 4$ to $127 \pm 5$ $\mathrm{mmHg}, \mathrm{p}<0.001)$ in women compared to men with PTSD. Diastolic $\mathrm{BP}(\mathrm{p}=0.799)$ and HR $(\mathrm{p}=0.670)$ responses to mental stress were not different. Similarly, MSNA reactivity $(\mathrm{p}=0.668)$ did not differ between the groups. Our preliminary findings show a sex difference in the hemodynamic response to mental stress in PTSD, with an exaggerated BP reactivity in women compared to men, suggesting a greater impact of PTSD on hypertension risk in women diagnosed with PTSD.

Funding: NIH HL-098744, NIH DK-00756, VA Merit I01CX001065, AHA 15CSA24340001.

\section{Plenary Lecture 2: Streeten Plenary Lecture}

\section{Multiple system atrophy and brainstem control of survival}

\section{E.E. Benarroch}

Mayo Clinic, Rochester, MN, USA

Multiple system atrophy (MSA) is a progressive neurodegenerative disease characterized by autonomic failure combined with parkinsonism, cerebellar ataxia, and pyramidal signs, in several combinations. Typical features of MSA are early severe orthostatic hypotension, neurogenic bladder, and sleep-related respiratory disorders including sleep apnea and laryngeal stridor. The pathological hallmark of MSA is the presence of $\alpha$-synuclein-containing oligodendroglial cytoplasmic inclusions and neuronal loss affecting the striatonigral and olivopontocerebellar systems as well as several autonomic areas. Recent studies in rodents using optogenetic and other approaches for selective activation or inactivation of specific groups of brainstem neurons have provided new insight into the mechanisms controlling cardiovascular function, respiration and wake-sleep cycle. Our neuropathological studies showed involvement of many of these brainstem areas in MSA, including the $\mathrm{C} 1$ group in the rostral ventrolateral medulla, serotonergic neurons in the medullary raphe, and neurons in the parabrachial nucleus. Many of these areas are involved in integrated control of blood pressure, respiration, arousal, and responses to hypoxia and hypercapnia. These findings thus provide for putative mechanistic substrates for life-threatening manifestations of MSA.

\section{Session 3: Awards Presentations}

\author{
FMS/Penaz Wesseling Travel Fellowship Award: Towards \\ improved hemodynamic criteria to distinguish between classic \\ orthostatic hypotension and reflex syncope during tilt- \\ table testing
}

\section{Ghariq, F.I. Kerkhof, R.H.A.M. Reijntjes, R.D. Thijs, J.G. van} Dijk

Department of Neurology, Leiden University Medical Centre, Leiden, The Netherlands

Introduction: Current criteria may not distinguish well between classic orthostatic hypotension (cOH) and vasovagal syncope (VVS) in tilt-table tests (TTT). We explored new haemodynamic features to improve the differentiation between $\mathrm{COH}$ and VVS.

Methods: TTT data were gathered from our Syncope Unit. Inclusion criteria for VVS were a history of classical VVS and TTT showing complaint recognition, a blood pressure (BP) drop and syncopal EEG changes. Inclusion criteria for $\mathrm{cOH}$ were a history of $\mathrm{cOH}$ and a $\mathrm{BP}$ decrease according to the 2011 consensus. Clinical diagnoses were established prior to TTT. Exclusion criteria were incomplete data, age $<16$ years, concurrent $\mathrm{cOH}$ and VVS and additional diagnoses. We defined (1) the pattern of the systolic BP decrease (i.e., whether the drop accelerated -'convex'- or decelerated -'concave'-; (2) time from tilt-up to reach half the maximum BP drop at tilt-back; (3) the difference between HR baseline and HR at BP nadir. We calculated the diagnostic value for these three features to differentiate between $\mathrm{cOH}$ and VVS. Results: We included 82 VVS cases (40\% male, median age 44) and $65 \mathrm{cOH}$ cases $(66 \%$ male, median age 70$)$. For $\mathrm{cOH}, 79 \%$ had a concave BP-pattern. For VVS, 94\% had a convex pattern $(\mathrm{p}<0.001)$. Half the BP drop was reached at $34 \mathrm{~s}$ for $\mathrm{cOH}$ and at $1571 \mathrm{~s}$ in VVS $(\mathrm{p}<0.001)$. Mean HR increased $11 \mathrm{bpm}$ in $\mathrm{cOH}$, whereas in VVS it decreased by $19.5 \mathrm{bpm}(\mathrm{p}<0.001)$. When all three features had to be present had a sensitivity of $82 \%$ for VVS and $71 \%$ for $\mathrm{cOH}$; specificity was $100 \%$ for VVS as well as for $\mathrm{cOH}$.

Conclusion: Three criteria help differentiate between $\mathrm{cOH}$ and VVS: shape of BP decline (convex for VVS, concave for $\mathrm{cOH}$ ); rate of drop (late in VVS, early in $\mathrm{cOH}$ ); HR response to BP drop (down in VVS, up or unchanged in $\mathrm{cOH}$ ). 
FMS/Penaz Wesseling Travel Fellowship Award: Effect of dietary salt intake on morning blood pressure surge in healthy women

R. Takeda, A.S. Stickford, J.-K. Yoo, R.A. Parker, M.A. RobertsReeves, Q. Fu

Institute for Exercise and Environmental Medicine, Texas Health Presbyterian Hospital Dallas, Dallas, TX, USA

Background: Salt intake may impact blood pressure regulation, but no study has investigated the effect of salt loading versus salt reduction on morning blood pressure (BP) surge which is considered to be an independent risk factor for hypertension and cardiovascular diseases. This study aimed to determine whether salt intake is related to morning BP surge in healthy women.

Methods: We measured 24-h ambulatory BP in 9 healthy women $[42 \pm 3(\mathrm{SD}) \mathrm{yr}]$ during a non-control diet $(\mathrm{CON})$ period. After that, subjects were given an isocaloric low-salt (LS; $50 \mathrm{mEq}$ sodium, 100 $\mathrm{mEq}$ potassium, and $1000 \mathrm{mg}$ calcium per day) and high-salt diet (HS; $250 \mathrm{mEq}$ sodium, $100 \mathrm{mEq}$ potassium, and $1000 \mathrm{mg}$ calcium per day) for 1 week each ( $\sim 2$-month apart with the order randomized). 24-h ambulatory BP was measured and blood sample and urine were collected following the LS and HS period. Morning BP surge was assessed as morning BP averaged for 2-h just after waking up (2-h awake BP) minus the lowest sleeping BP.

Results: Awake, asleep and overall 24-h BPs were not different between the three conditions; however, morning systolic BP surge was higher in LS than CON ( $32 \pm 7$ vs. $18 \pm 9 \mathrm{mmHg}$, $\mathrm{p}=0.024)$ but not HS (vs. $25 \pm 10 \mathrm{mmHg}, \mathrm{p}=0.467$ ). Morning systolic BP surge was positively correlated with 2 -h awake $\mathrm{BP}(\mathrm{r}=0.82, \mathrm{p}=0.007)$ in $\mathrm{LS}$ and $\operatorname{CON}(r=0.61, p=0.080)$ but not in HS $(r=0.27, p=0.485)$. The percent change in plasma volume from LS to HS was $6.9 \%(\mathrm{p}=0.006)$ and 24-h urine output, osmolality, and urine sodium level were all lower in LS than in HS (all, $\mathrm{p}<0.05$ ).

Conclusion: Morning blood pressure surge is increased and is positively correlated with 2-h awake blood pressure during salt reduction, but this relationship disappears during salt loading in healthy women. The underlying mechanisms are unknown, but may be attributed, in part, to sympathetic activation.

Funding: Harry S. Moss Heart Trust.

\section{Streeten Travel Fellowship Award: Increased gastrointestinal gut hormones secretion following a glucose challenge in postural orthostatic tachycardia syndrome}

N.C. Breier, S.Y. Paranjape, S. Scudder, S. Lonce, S.E. Mehr, A. Diedrich, C.R. Flynn, C.A. Shibao

Department of Clinical Pharmacology, Vanderbilt University Medical Center, Nashville, TN, USA

Background and Methods: Obesity-associated increased sympathetic activation (SNS) has been associated with negative metabolic outcomes. The purpose of this study was to evaluate whether SNS activation, in the absence of obesity, is associated with altered gastrointestinal (GI) gut hormones secretion (insulin, C-peptide, incretin hormones (GLP-1, GIP), pancreatic polypeptide (PP), peptide YY (PYY), and Glucagon) known to regulate glucose homeostasis. We studied 12 lean POTS patients, a condition characterized by increased SNS, and 13 healthy controls (HC). Patients were matched by age ( $37 \pm 12$ vs. $30 \pm 8$ years in $\mathrm{HC}, \mathrm{P}=0.11)$ and $\mathrm{BMI}(24 \pm 4.5$ vs. $23 \pm 3.2$ $\mathrm{kg} / \mathrm{m}^{2}$ in $\mathrm{HC}, \mathrm{P}=0.36$ ). As expected, POTS patients had higher upright heart rate compared to $\mathrm{HC}(118 \pm 13$ vs. $86 \pm 14 \mathrm{bpm}, \mathrm{P}=0.002)$ and elevated upright norepinephrine (NE, $835.2 \pm 368$ vs. $357 \pm 157 \mathrm{pg} / \mathrm{mL}$ in $\mathrm{HC}, \mathrm{P}=0.001)$. All subjects underwent a 75 -gr oral glucose test mixed with $20 \mathrm{mg} / \mathrm{kg}$ acetaminophen to assess for gastric emptying with early time-points $(0,5,10,15,30,60,90$, and 120 min postglucose challenge).

Results: Both groups had normal fasting and post-intake glucose excursion. Acetaminophen blood levels were similar between groups ( $78 \pm 14.1$ vs. $74 \pm 32.9 \mathrm{ug} / \mathrm{mL}$ in $\mathrm{HC}, \mathrm{P}=0.77$ ), suggesting normal gastric emptying. POTS patients had significant increase in C-peptide ( $4064 \pm 950$ vs. $2321 \pm 478, \mathrm{P}<0.001)$, GIP $(75.7 \pm 41.8$ vs $42.7 \pm 9.6$ in $\mathrm{HC}, \mathrm{pmol} / \mathrm{L}, \mathrm{P}<0.001), \mathrm{PP}(146.3 \pm 104.3$ vs. $111.24 \pm 64$ in $\mathrm{HC}, \mathrm{pg} /$ $\mathrm{mL}, \mathrm{P}=0.05)$, and PYY ( $188 \pm 88$ vs. $205 \pm 107, \mathrm{P}=0.04)$. GLP-1 and glucagon levels were similar between groups. Matsuda index of insulin sensitivity was lower in POTS ( $3.9 \pm 1.0$ vs. $5.1 \pm 1.3$ in $\mathrm{HC}$, $\mathrm{P}=0.02)$. Only GIP peak levels were positively associated with $\mathrm{NE}$ levels ( $\mathrm{r}=0.6, \mathrm{P}=0.016)$. In conclusion, in the absence of obesity, SNS activation in POTS is associated with impaired metabolic profile with decreased insulin sensitivity and increased secretion of GIP, PP, PYY and C-peptide.

Funding: The study was supported by gift funds from a private donation, Dysautonomia International 266 Foundation, and NIH grants DK059637 (MMPC) and DK020593 (DRTC).

\section{Friday, November 8, 2019}

\section{Plenary Lecture 3: Hot Topic Plenary Lecture}

\section{The gut microbiome: all hype or true hope?}

\section{A. Zarrinpar}

Center for Microbiome Innovation, Division of Gastroenterology, University of California, San Diego, La Jolla, CA, USA

Interest in the gut microbiome is at an all-time high, with the microbiome connected to an increasing number of diseases including neurological diseases. Though our understanding of the gut microbiota-brain axis is at its infancy, there is evidence that the autonomic nervous system plays an important role in mediating these signals. There has been enormous progress in the past decade in using genomic sequencing coupled with computational pipelines to decipher how the gut microbiome can cause or modify disease. However, apart from the 1700-year-old technique of stool transplantation for the treatment of recurrent/resistant Clostridium difficile infection, it is unclear whether therapies that target microbiome composition (e.g., probiotics, prebiotics) can be translated to the clinic or are robust enough to temper the great interpersonal diversity and plasticity of human hosts. Moreover, the lack of reproducibility in many microbiome studies have highlighted that many studies have been relying on key assumptions that have now been proven false. New tools are needed to move beyond correlative studies and to functionally link features of the gut microbiome with host physiological processes. This talk will highlight the current limitations and future promise of gut microbiome research, with the expectation that an understanding of these considerations will help accelerate the path toward routine clinical application of microbiome-mediated therapies. 


\section{Session 4: Orthostatic Hypotension and Supine Hypertension}

\section{A phase 2 study of the efficacy, durability, and safety of ampreloxetine (TD-9855), a norepinephrine reuptake inhibitor, given once-daily to treat neurogenic orthostatic hypotension $(\mathrm{nOH})$ in subjects with synucleinopathies}

\begin{abstract}
H. Kaufmann ${ }^{1}$, I. Biaggioni ${ }^{2}$, A. Panneerselvam ${ }^{3}$, B. Haumann ${ }^{3}$, R Vickery ${ }^{4}$

${ }^{1}$ Department of Neurology, New York University School of Medicine, New York, NY, USA; ${ }^{2}$ Department of Medicine, Vanderbilt University Medical Center, Nashville, TN, USA; ${ }^{3}$ Theravance Biopharma US, Inc., South San Francisco, CA, USA; ${ }^{4}$ Theravance Biopharma Ireland Limited, Dublin, Ireland
\end{abstract}

Objective: To assess efficacy and durability (using Orthostatic Hypotension Symptom Assessment [OHSA] and OH Daily Activities Scale [OHDAS]), and safety of ampreloxetine, once-daily to treat neurogenic orthostatic hypotension $(\mathrm{nOH})$ in subjects with synucleinopathies.

Background: Failure of the autonomic nervous system results in $\mathrm{nOH}$ with inadequate increase in synaptic norepinephrine (NE) and maintenance of upright blood pressure (BP) following postural change. Ampreloxetine is a novel norepinephrine reuptake inhibitor being investigated for treatment of symptomatic $\mathrm{nOH}$.

Methods: In a 24-week open-label phase 2 multicenter study of subjects with $\mathrm{nOH}$, following a single-dose escalation phase, responders were treated with oral ampreloxetine (3-20 mg) once-daily for up to 20 weeks with 4-week follow-up after treatment withdrawal (Week 24). Primary efficacy endpoint OHSA\#1 (dizziness, lightheadedness, feeling faint) was assessed at end of Week 4; end of open-label treatment (Week 20); and after treatment withdrawal (Week 24).

Results: Twenty-one subjects enrolled in the open-label phase (mean age, 64 years; 57\% multiple-system atrophy). Sixteen (76\%), 11 (52\%), and $10(48 \%)$ subjects completed assessments at Weeks 4, 20, and 24, respectively. Mean (SD) improvement from baseline in OHSA\#1 was 2.4 (4.5) and 1.9 (3.1) for all subjects, and 3.8 (3.1) and 3.1 (3.0) for symptomatic subjects (OHSA\#1 $\geq 4$ at baseline) at Weeks 4 and 20, respectively, scores were back to baseline levels at Week 24. Similar trends in improvement were observed in OHSA and OHDAS composite scores. Most common adverse events (AEs) were urinary tract infection (24\%), hypertension (19\%), and headache $(14 \%)$. Two subjects (10\%) discontinued treatment due to AEs and 5 (24\%) reported serious AEs, none considered related to study medication.

Conclusion: In subjects with $\mathrm{nOH}$, ampreloxetine demonstrated clinically meaningful improvement in OHSA\#1 at Week 4, which was sustained over 20 weeks, and reverted to baseline after treatment withdrawal. Similar trends were observed in OHSA and OHDAS composite scores. Ampreloxetine was well-tolerated.

Funding: Theravance Biopharma.

\section{Local passive heat in the treatment of nocturnal supine hypertension in autonomic failure}

L.E. Okamoto ${ }^{1}$, J.E. Celedonio ${ }^{1}$, E.C. Smith ${ }^{1}$, A. Gamboa ${ }^{1}$, C.A. Shibao $^{1}$, A. Diedrich ${ }^{1}$, S. Paranjape ${ }^{1}$, B.K. Black ${ }^{1}$, J.A. Muldowney $3 \mathrm{rd}^{2}$, A.C. Peltier ${ }^{1,3}$, R. Habermann ${ }^{1}$, C.G. Crandall ${ }^{4}$, I. Biaggioni ${ }^{1}$ Departments of ${ }^{1}$ Medicine, ${ }^{2}$ Cardiology and ${ }^{3}$ Neurology, Vanderbilt University Medical Center, Nashville, TN, USA; ${ }^{4}$ Institute for Exercise and Environmental Medicine, Texas Health Presbyterian Hospital and UT Southwestern Medical Center, Dallas, TX, USA
Supine hypertension affects about half of patients with primary autonomic failure (AF), complicates the treatment of orthostatic hypotension $(\mathrm{OH})$ and induces nocturnal polyuria, resulting in worsening of morning $\mathrm{OH}$. We previously showed that short exposure to local passive heat $\left(40-42^{\circ} \mathrm{C}\right.$ applied for $\leq 2$ hours over abdomen and pelvis with a heating pad) acutely decreased systolic blood pressure (BP) by $-25 \pm 5 \mathrm{mmHg}$ in $\mathrm{AF}$ patients with supine hypertension due to decreases in stroke volume $(-29 \pm 5 \%)$ and cardiac output $(-30 \pm 5 \%)$. In this study, we hypothesized that local passive heat therapy applied overnight would lower nocturnal BP and polyuria, and improve early morning $\mathrm{OH}$ in these patients. Ten $\mathrm{AF}$ patients with supine hypertension (age $76 \pm 2$ years, 6 men, supine systolic BP $168 \pm 3 \mathrm{mmHg})$ received passive heat $\left(38^{\circ} \mathrm{C}\right.$ with a waterperfused heating pad placed under the torso) or placebo, in a 2-night crossover study. Supine BP was monitored every $2 \mathrm{hr}$ from $8 \mathrm{pm}-8$ am. Heat therapy was applied from 10 pm-6 am. Morning orthostatic tolerance was assessed at $8 \mathrm{am}$. Supine systolic BP significantly decreased during overnight heat therapy compared to placebo $(\mathrm{P}<0.01$ by mixed-effects model) with a maximal reduction of $30 \pm 6$ $\mathrm{mmHg}$ at $4 \mathrm{hr}$ of heat (vs. $-8 \pm 5 \mathrm{mmHg}$ for placebo; $\mathrm{P}<0.01$ ). Despite lowering nighttime BP, heat therapy did not decrease nocturnal diuresis or improve morning $\mathrm{OH}$. In conclusion, low levels of local heat therapy applied overnight effectively lowers BP in AF patients with nocturnal supine hypertension. Thus, local heat therapy offers a novel non-pharmacologic approach to treat this condition, but future studies are needed to assess the long-term safety of this approach. Funding: NIH grants U54 NS065736, R01 HL122847 and UL1 TR002243 (CTSA award).

Changes in supine blood pressure with long-term droxidopa use

L.A. Hewitt ${ }^{1}$, S. Gorny ${ }^{1}$, A. Lindsten ${ }^{2}$, M. Karnik-Henry ${ }^{1}$, S. Kymes ${ }^{3}$, A. Favit ${ }^{1}$

${ }^{1}$ US Medical, Lundbeck, Deerfield, IL, USA; ${ }^{2}$ Biostatistics, Lundbeck A/S, Copenhagen, Denmark; ${ }^{3}$ Health Economic and Outcomes

Research, Lundbeck, Deerfield, IL, USA

Objective: To determine the long-term effects of droxidopa use on supine blood pressure (BP) in patients with neurogenic orthostatic hypotension $(\mathrm{nOH})$.

Background: Because of baroreflex dysfunction, patients with nOH may also experience supine hypertension. Pressor agents used to treat $\mathrm{nOH}$, such as droxidopa, may increase standing and supine BP.

Methods: BP changes over time were examined during a 12-month, long-term extension study of droxidopa (100-600 mg, 3 times daily). These analyses focused on patients who transitioned to/from "high" supine systolic BP (SBP), defined as $>140 \mathrm{mmHg}$, based on classification of BP measurements obtained before treatment initiation (baseline) and at study visits.

Results: Data from 102 patients were analyzed. At baseline, overall mean \pm SEM supine SBP was $132 \pm 2 \mathrm{mmHg}$, with baseline groupings of low $(97 \pm 2 \mathrm{mmHg}, \mathrm{n}=15)$, normal $(125 \pm 1 \mathrm{mmHg}, \mathrm{n}=49)$, and high $(155 \pm 2 \mathrm{mmHg}, \mathrm{n}=38)$. In the combined group of patients with low/normal supine SBP at baseline $(119 \pm 2 \mathrm{mmHg}, \mathrm{n}=64), 29 \%(12 /$ $41)$ and $30 \%(10 / 33)$ transitioned to high supine SBP after 6 and 12 months of treatment, respectively (mean, $155 \pm 3$ and $162 \pm 4 \mathrm{mmHg}$; maximum, 171 and $187 \mathrm{mmHg}$ ). Among patients with high supine SBP at baseline, 38\% (10/26) and 38\% (9/24) transitioned to low/ normal supine SBP at 6 and 12 months (mean, $127 \pm 4$ and $129 \pm 4$ $\mathrm{mmHg}$ ). Supine SBP changes from baseline to 12 months in the baseline low/normal and high supine SBP groups were $+12 \pm 4$ and $3 \pm 6 \mathrm{mmHg}$, respectively. Supine hypertension, defined as supine SBP $>180 \mathrm{mmHg}$, was observed in 14\% (14/102) of patients at any time during the study (baseline low/normal, 5\%; baseline high, 29\%). 
Conclusion: In this study, approximately one-third of patients saw potentially clinically important SBP shifts. Because patients treated with droxidopa can experience BP increases (due to pressor activity) or decreases (due to therapeutic effects), monitoring and evaluating BP changes during treatment are important.

Funding: The data reported were derived from a clinical trial funded by Lundbeck. All authors are employees of Lundbeck.

Best Basic Science Autonomic Research: Bezold-Jarisch reflex and beyond: differential engagement of inhibitory and excitatory cardiopulmonary reflexes by capsaicin and phenylbiguanide

\section{R.A. Larson, M.W. Chapleau}

Internal Medicine, University of Iowa, Iowa City, IA, USA

The Bezold-Jarisch reflex is a powerful inhibitory reflex initiated by activation of cardiac vagal afferent nerves during orthostatic stress and/or myocardial ischemia leading to bradycardia, vasodilation, hypotension and vasovagal syncope. This clinically relevant reflex has been studied by measuring heart rate (HR) and mean arterial pressure (MAP) responses to intravenous (IV) injections of a variety of chemical compounds known to activate cardiac sensory nerves. We hypothesized that reflex responses to compounds may vary due to differential activation of vagal afferent subtypes and/or variable coactivation of excitatory afferents. HR and MAP responses to IV injections of the TRPV1 agonist capsaicin ( 25 and $50 \mathrm{ng} / \mathrm{g}, \mathrm{n}=6)$ and the serotonin 5-HT3 receptor agonist phenylbiguanide (PBG, 50 and $100 \mathrm{ng} / \mathrm{g}, \mathrm{n}=5$ ) were measured in anesthetized C57BL/6 mice with vagus nerves intact and again after bilateral cervical vagotomy. Capsaicin and PBG each evoked rapid dose-dependent decreases in HR and MAP followed by small, significant increases in HR and MAP above baseline $(\mathrm{P}<0.05)$. The rapid Bezold-Jarisch reflex responses were abolished after vagotomy, while the delayed tachycardia and pressor responses were enhanced. The relative magnitude of reflex bradycardic vs. depressor responses $(\downarrow$ HR, $/$ MAP) in vagusintact mice was greater $(\mathrm{P}<0.05)$ with capsaicin $(-133 \pm 21 \mathrm{bpm}$, $28.7 \pm 3.1 \mathrm{mmHg}$, ratio $=4.6 \pm 0.5)$ than with $\mathrm{PBG}(-67 \pm 17 \mathrm{bpm}$, $27.1 \pm 5.0 \mathrm{mmHg}$, ratio= $2.4 \pm 0.3)$. In contrast, after vagotomy the magnitude of excitatory reflex tachycardic vs. pressor responses $(\uparrow \mathrm{HR} / \uparrow \mathrm{MAP})$ was less $(\mathrm{P}<0.05)$ with capsaicin than with $\mathrm{PBG}$. We conclude: (1) Capsaicin and PBG (IV) each activate the Bezold-Jarisch reflex in mice, with the contribution of bradycardia to the decrease in MAP being greater for capsaicin; and (2) Activation of excitatory reflexes contribute to responses to both drugs, most notably for capsaicin. These results have implications for identifying endogenous agonists of TRPV1 and 5-HT3 receptors and their potential for triggering cardioinhibitory and/or vasodilator reflexes in physiological and pathophysiological states.

Funding: NIH HL14388, NIH F32HL140880.

Can urine sodium concentration be determined through chloride test strips?

N.D. Heeney, B.C.D. Hockin, S. Sanatani, K. Armstrong,

V.E. Claydon

Department of Biomedical Physiology and Kineisiology, Simon Fraser University, Burnaby, BC, Canada

Introduction: Syncope diagnosis is challenging and often requires numerous tests with multiple physicians. Individuals with syncope tend to have low sodium intake, and often benefit from increases in dietary sodium. Sodium intake and compliance with dietary salt supplementation can be inferred from 24-hour urine collection, but this is inconvenient, particularly in children. Urine spot samples have been investigated as an alternative to 24-hour collections, but the accuracy of a single spot sample is poor. Since sodium and chloride are present in equal concentrations in dietary salt, chloride test strips may provide a suitable proxy for urine sodium concentration. We aimed to determine if: (i) chloride test strips provide a reliable measure of urinary sodium compared to the gold standard of flame photometry; (ii) multiple spot samples provide an accurate estimate of 24-hour urine sodium.

Methods: We recruited 23 participants (11 males) aged 23.3 \pm 0.9 (range 19-35) years to complete multiple consecutive spot samples (morning and evening) along with a 24-hour urine sodium collection on the middle day.

Results: Urine 24-hour sodium estimates using chloride test strips $(109 \pm 8.6 \mathrm{mmol} / \mathrm{day})$ were highly correlated with flame photometry (120 $\pm 10.0 \mathrm{mmol} / \mathrm{day})$ measurements $(\mathrm{r}=0.897, \mathrm{p}<0.001)$, with Bland-Altman analyses determining a bias of $-10.6 \pm 21.2 \mathrm{mmol} /$ day. Use of chloride tests strip for evaluation of multiple morning spot samples as a proxy for 24-hour sodium gave similar results whether samples from three, four or five days were used $(\mathrm{p}=0.995)$. Use of evening spot samples gave more reliable estimates of 24-hour urine sodium than morning samples. Estimates of 24-hour urine sodium with chloride test strips from three evening spot samples (with a correction factor applied) (128 $\pm 9.8 \mathrm{mmol} /$ day) provided a good approximation of 24-hour urine collection $(131 \pm 12.3 \mathrm{mmol} / \mathrm{day})$, with a bias of $-3.10 \pm 39.9 \mathrm{mmol} /$ day.

Conclusion: Chloride strips provide a comfortable and feasible opportunity for patients with syncope to monitor dietary sodium supplementation for the treatment of syncope.

Funding: Heart and Stroke Foundation G-18-00221.

\section{Symposium 3: Vasovagal Syncope: The Present and the Future}

Vasovagal syncope (VVS) is the most common cause of fainting across all age groups. The majority of patients with VVS can manage their syncope with dietary salt and water expansion and by learning physical counter maneuvers. However, there is a subset of patients with frequent and recurrent fainting who may benefit from pharmacological management of their VVS. In this session, we will review the evidence basis for some medications that are commonly used to treat VVS.

For patients with frequent and refractory vasovagal syncope, permanent pacemakers have been suggested as a useful therapy. This treatment has undergone cycles of enthusiasm and disappointment, and now seems to again be on the ascent. We will review the current state of this therapy.

An emerging therapy is the use of radiofrequency ablation in the heart to treat recurrent vasovagal syncope, including a summary of the techniques, outcomes and assessment of the future role of this potentially exciting technique in improving symptoms in vasovagal syncope. While still in its infancy, we will review the putative mechanisms and the early data for such an approach.

The physiology of neuro-cardiogenic syncope in children is not well studied compared to adults' literature. Finally, we will focus on the special considerations required to evaluate and manage a child or adolescent with syncope, including cerebral perfusion, cardiac stroke volume, Baro-receptors activity and sympathetic/parasympathetic tone. 


\section{Symposium 4: Heart Failure: Cutting Edge Autonomic Approach}

\section{Carotid body contribution to autonomic disorders}

\section{R. Iturriaga}

Lab Neurobiology, Department of Physiology, Pontificia Universidad Católica de Chile, Santiago, Chile.

The carotid body (CB) has been involved in sympathetic mediated diseases such as obstructive sleep apnea (OSA), systolic heart failure (HF), and cardiometabolic diseases. Although the main pathological disease associated with the $\mathrm{CB}$ are the chemodectomas, a growing body of new experimental evidences support that an abnormal enhanced $\mathrm{CB}$ chemosensory discharge triggers central autonomic dysfunction, a common feature of these diseases. The enhanced chemosensory discharge produces sympathetic overactivation, impairs baroreflex sensitivity, produces alterations of heart rate variability, breathing instability, insulin resistance and hypertension. The mechanisms underlaying the potentiation of $\mathrm{CB}$ chemosensory discharge are not completely known. However, oxidative stress, inflammation, increased levels of ET-1 and Ang II and reduction of NO levels in the CB are involved in the chemosensory potentiation. The bilateral ablation of the CBs or the carotid sinus neurotomy reduces the sympathetic hyperactivation, the elevated arterial pressure in OSA and hypertensive models, reduces breathing instability and improves animal survival in HF preclinical models, and restores insulin tolerance in animals exposed to rich carbohydrate and fact diets, highlighting the importance of the enhanced $\mathrm{CB}$ discharge in the progression of these sympathetic-related diseases and supports the idea that the elimination of CB input may be useful to improve cardiovascular and endocrine alterations. Therefore, the $\mathrm{CB}$ ablation has been recently used in pilot human studies as a therapeutic treatment for resistant hypertension and HF-induced sympathetic hyperflow. In this symposium, I will discuss the evidence for the crucial role played by $\mathrm{CB}$ in the autonomic dysfunction, and the effectiveness of the $\mathrm{CB}$ ablation in humans.

\section{Molecular basis of cardiac mechanosensory and chemosensory signaling: implications in heart failure}

\section{M.W. Chapleau}

Departments of Internal Medicine \& Molecular Physiology and Biophysics, University of Iowa, and Veterans Affairs Medical Center, Iowa City, IA, USA

The heart is densely innervated by autonomic, intrinsic and sensory neurons. The sensory nerves travel in vagal and spinal 'sympathetic' afferents with cell bodies located in nodose and dorsal root ganglia, respectively. In general, vagal afferents are sympathoinhibitory and spinal afferents are sympathoexcitatory (with directionally opposite effects on parasympathetic activity), although there are exceptions. Diverse subtypes of cardiac sensory neurons exist including mechanosensitive, chemosensitive and polymodal afferents. In my presentation I will (1) briefly review the roles of cardiac afferent reflexes in health and disease; (2) summarize recent discoveries that define the vast molecular/functional diversity of subtypes of vagal and spinal afferent neurons; (3) describe molecular mechanisms of activation and modulation of cardiac afferents, and the dysregulation that occurs in heart failure; and (4) discuss the reflex consequences of abnormal cardiac afferent activity in heart failure, and novel therapeutic approaches targeting cardiac afferents.
Splanchnic modulation in heart failure

M. Fudim

Division of Cardiology, Duke University, Duke Clinical Research Institute, Durham, NC, USA

The key hallmark of heart failure (HF) is vascular congestion with resultant limitation in physical activity. The current HF pathophysiologic model suggests that congestion is the result of volume retention; therefore therapies (such as diuretics and ultrafiltration) have generally been targeted at volume overload. Despite this, the therapeutic benefits of fluid removal strategies on short- and longterm outcomes have been modest or absent. More recently the concept of fluid gain as cause of cardiac decompensation has been challenged. Despite evidence of elevated intra-cardiac pressures, as many as $50 \%$ of patients with $\mathrm{HF}$ do not experience fluid/weight gain prior to a HF hospitalization. To reconcile these findings, a new hypothesis of cardiac congestion and decompensation was proposed. The hypothesis is that cardiovascular congestion may be the result of blood volume redistribution from the abdominal (splanchnic) compartment to the heart and lungs. This hypothesis arises from the fact that the autonomic nervous system is the main determinant of blood volume distribution in the body. Preliminary work has identified the greater splanchnic nerve (GSN) as a novel target to treat HF. In a series of two small first-in-human studies for acute decompensated $\mathrm{HF}(\mathrm{N}=13)$ and chronic $\mathrm{HF}(\mathrm{N}=17)$, we found that a splanchnic nerve block (SNB) with lidocaine (90 min duration of action) and ropivacaine (24 hours duration of action) acutely reduced resting and exercise-induced intra-cardiac filling pressures, associated with improved patient symptoms and functional capacity. Targeting the splanchnic autonomic nervous system to treat HF may present a potential therapeutic target not currently utilized for HF care. Continued efforts to investigate short- and long-term effects of SNB in chronic HF are warranted.

\section{Symposium 5: Gastrointestinal System in Autonomic Disorders}

\section{Enteric nervous system: the big brain!}

\section{K.A. Sharkey}

Hotchkiss Brain Institute, Department of Physiology and Pharmacology, Cumming School of Medicine, University of Calgary, Calgary, AB, Canada

The enteric nervous system (ENS) is the third division of the autonomic nervous and the only division that is really autonomous from the CNS. It innervates the entire gastrointestinal tract, regulating the musculature, secretion of fluid and local blood flow. Digestion is controlled through the integration of signals from the ENS and CNS, as well as between distinct gut regions via the autonomic ganglia to coordinate digestive activity. Consisting of enteric neurons and a unique type of peripheral glial cell, the ENS is organized in two major ganglionated plexuses; the myenteric plexus which is located between the external muscle layers and the submucosal plexus that lies in the submucosa. Neurons in enteric reflex pathways use a wide range of chemical transmitters that signal through a variety of receptors.

The epithelial lining of the gastrointestinal tract serves as the interface for digestion and absorption of nutrients and water and as a defensive barrier. The defensive functions of the intestinal epithelium are remarkable considering that the gut lumen is home to trillions of resident bacteria, fungi and protozoa (collectively, the intestinal microbiota) that must be prevented from translocation across the epithelial barrier. Imbalances in the relationship between the 
intestinal microbiota and the host contribute to the manifestation of diseases that range from disorders of motility and sensation (e.g., irritable bowel syndrome) and intestinal inflammation (e.g., inflammatory bowel disease) to behavioural and metabolic disorders, including autism and obesity. In addition to controlling the digestive functions of the gut, the ENS plays critical roles in host defense by integrating epithelial, enteroendocrine and immune functions to maintain homeostasis.

Termed the "little brain" by some authors, the ENS is in fact a remarkably complex and substantive component of the autonomic nervous system that serves the dual roles of controlling digestion and host defense as outlined above and hence in reality it is "a big brain" lying in the wall of the gut.

\section{Evaluation of gastrointestinal problems in dysautonomia}

\section{G. Chelimsky}

Medical College of Wisconsin, Milwaukee, WI, USA

Gastrointestinal symptoms are often present in autonomic disorders. Often, they are secondary to the autonomic dysfunction, but in the functional dysautonomias, more frequently the gastrointestinal symptoms are just comorbid conditions. In organic dysautonomias such as diabetes, familial dysautonomia, amyloidosis, Parkinson's disease, the gastrointestinal disorders are produced by direct involvement of the autonomic nervous system. In the functional dysautonomias, such as postural tachycardia syndrome (POTS), only the symptoms replicated during the orthostatic challenge are the ones produced by the autonomic dysfunction. All the other symptoms that can happen supine or do not improve in the supine position, are not secondary to the dysautonomias, but are just part of the newly defined symptom by the National Institute of Health (NIH) as Chronic Overlapping Pain Condition (COPC). In this last case, treatment aimed at the POTS will not improve the symptoms. Only in the case of functional dysautonomias in which the symptoms are replicated by orthostatic challenge such as in the case of POTS, these symptoms will improve with treatment aimed at the orthostatic challenge. In contrast, in organic dysautonomias usually there is a direct relationship between the degeneration or injury to the autonomic nerves or centers and the gastrointestinal symptoms. The most common gastrointestinal symptoms present in all subgroups of dysautonomias include dysphagia, early satiety, nausea, vomiting and weight loss, usually originating from the foregut. Sometimes the symptoms are secondary to small intestine bacterial overgrowth and they can present with bloating, flatus, malabsorption, etc. The most common symptoms in the lower gastrointestinal tract include constipation, diarrhea and fecal incontinence. The evaluation will depend on the symptoms and include blood work, stool samples, gastric emptying studies, hydrogen breath test, upper and lower endoscopy, motility studies, transit time studies, contrast studies of the upper gastrointestinal tract or barium enemas, and sometimes ultrasound of the celiac artery. Management will depend on the findings and on the symptoms.

\section{Management pearls for gastrointestinal problems in autonomic disorders}

\section{J.E. Fortunato}

Neurointestinal \& Motility Program, Northwestern University Feinberg School of Medicine, Pediatric Gastroenterology, Hepatology, and Nutrition, Ann \& Robert H. Lurie Children's Hospital of Chicago, Chicago, IL, USA

Autonomic disorders are often associated with gastrointestinal symptoms such as abdominal pain and nausea as well as co- morbidities including anxiety. These symptoms are often heterogeneous in their presentation with their causes multifactorial. They have varied and numerous precipitating triggers, making it difficult to determine etiology and optimal treatment strategies. When routine diagnostic testing fails to identify a cause for the gastrointestinal symptoms, they are often defined as functional in nature. For this reason, patients are treated with empiric, costly, and not patientspecific therapy in an attempt to alleviate their symptoms. To better define symptoms and determine the relationship between the GI tract and the autonomic nervous system necessitates an integrated, and frequently, multidisciplinary approach versus isolated assessment of each symptom. This includes incorporation of the biopsychosocial model. Recognizing, characterizing, assessing the complex interaction among symptoms is a first step toward the development of treatments. Therefore, use of assessment tools such as symptom questionnaires examining all symptom domains - autonomic, GI, and psychological aspects - may assist in providing better understanding of patient phenotypes and more directed, evidenced based treatments.

Saturday, November 9, 2019

\section{Plenary Lecture 4: MSA Plenary Lecture}

\section{The early stages of synculeinopathies: is prodromal MSA detectible?}

\section{R. Postuma}

McGill University, Montreal, QC, Canada

Over the last 15 years, there have been dramatic advances in the field of prodromal synucleinopathy. By combining motor markers, cognitive changes, special sensory, and sleep markers, we are now able to identify patients with prodromal synucleinopathy, sometimes with near-complete certainty. Biomarkers, especially neuroimaging and biopsy markers are catching up, and can make diagnoses even certain. This talk will overview the field of prodromal synucleinopathy, with a special focus on multiple system atrophy

\section{Session 5: MSA and Other Degenerative Diseases}

Don Summers Memorial MSA Travel Award: Baseline characteristics of patients with multiple system atrophy enrolled in the Natural History Study of the Synucleinopathies

M.A. Perez ${ }^{1}$, J.-A. Palma ${ }^{1}$, L. Norcliffe-Kaufmann ${ }^{1}$, W. Singer ${ }^{2}$, P. Low ${ }^{2}$, M.T. Pellecchia ${ }^{3}$, H.-J. Kim ${ }^{4}$, C. Shibao ${ }^{5}$, A. Peltier ${ }^{5}$, I. Biaggioni ${ }^{5}$, D. Giraldo ${ }^{6}$, M.J. Marti ${ }^{6}$, A. Fanciulli ${ }^{7}$, C. Terroba ${ }^{8}$, M. Merello ${ }^{8}$, D.S. Goldstein ${ }^{9}$, R. Freeman ${ }^{10}$, C.H. Gibbons ${ }^{10}$, S. Vernino ${ }^{11}$, F. Krismer ${ }^{7}$, G. Wenning ${ }^{7}$, H. Kaufmann ${ }^{1}$; on behalf of the Natural History Study of the Synucleinopathies Study Group

${ }^{1}$ Department of Neurology, New York University School of Medicine, New York, NY, USA; ${ }^{2}$ Department of Neurology, Mayo Clinic, Rochester, MN, USA; ${ }^{3}$ Department of Neurology, Salerno University, Salerno, Italy; ${ }^{4}$ Department of Neurology, Seoul National University, Seoul, South Korea; ${ }^{5}$ Department of Medicine and Pharmacology, Vanderbilt University, Nashville, TN, USA;

${ }^{6}$ Movement Disorders Unit, Neurology Service, Hospital Clínic de Barcelona, Institute of Neuroscience, University of Barcelona, Barcelona, Spain; ${ }^{7}$ Department of Neurology, Innsbruck Medical University, Innsbruck, Austria; ${ }^{8}$ Movement Disorders Division, FLENI, Buenos Aires, Argentina; ${ }^{9}$ Neurocardiology Section, Intramural NINDS, Bethesda, MD, USA; ${ }^{10}$ Department of Neurology, 
Beth Israel Deaconess Medical Center, Boston, MA, USA;

${ }^{11}$ Department of Neurology, University of Texas Southwestern, Dallas, TX, USA

Background: Multiple system atrophy (MSA) is a fatal and poorly understood rare neurodegenerative disorder. Here we describe the baseline characteristics of patients with MSA enrolled in a prospective multicenter and multinational NIH-sponsored Natural History Study of the Synucleinopathies.

Methods: Patients with a clinical diagnosis of probable or possible MSA were prospectively enrolled at 11 participating centers. Demographic data, clinical variables, and autonomic testing results were included.

Results: 293 patients with MSA (125 women) have been enrolled. MSA-C was predominant (154 patients, 52.6\%). Mean age at symptom onset was $57.6 \pm 8.4$ (mean \pm SD) and at enrollment was $62.0 \pm 7.8$ years old. UMSARS-1 was $21.1 \pm 7.6$ and UMSARS-2 was $21.2 \pm 9.1$. MoCA score was $26.3 \pm 4.4$ indicating normal cognition. In the supine position, blood pressure (systolic BP/diastolic BP) was $143.0 \pm 25.2 /$ $84.0 \pm 14.5 \mathrm{mmHg}$, and heart rate was $75.0 \pm 11.5 \mathrm{bpm}$. After 3-min head-up tilt, BP fell to $113.1 \pm 25.5 / 69.6 \pm 15.9 \mathrm{mmHg}$ and $\mathrm{HR}$ increased to $82.9 \pm 12.7 \mathrm{bpm}$. Supine plasma norepinephrine levels were $365.4 \pm 408.5 \mathrm{pg} / \mathrm{ml}$ and increased only to $449.8 \pm 277.2 \mathrm{pg} / \mathrm{ml}$ upon head-up tilt indicating impaired baroreflex-mediated sympathetic activation. The University of Pennsylvania Smell Identification Test (UPSIT) score was $28.5 \pm 8.1$ indicating preserved olfaction. Probable rapid eye movement (REM) sleep behavior disorder was reported by $85 \%$.

Conclusions: This is the largest cross-sectional sample of patients with MSA recruited consecutively reported so far. Our results confirm that: i) symptom onset in MSA is remarkable consistent at 57 years; ii) overt cognitive impairment is not a typical feature; iii) sympathetic and cardiovagal deficits are present; iv) olfaction is preserved, and; v) probable REM behavior disorder is very frequent. The prospective follow-up of these patients will provide additional information on the natural history of the disease.

Funding: MSA Coalition and NIH (U54-NS065736-01).

\section{Predicting evolution in pure autonomic failure}

E.A. Coon, S.E. Berini, J.N. Mandrekar, E.E. Benarroch,

P. Sandroni, P.A. Low, W. Singer

Department of Neurology, Mayo Clinic, Rochester, MN, USA

Objective: To determine the frequency and predicting factors of evolution from pure autonomic failure (PAF) into a synucleinopathy with motor or cognitive involvement of multiple system atrophy (MSA), Parkinson disease (PD), or dementia with Lewy bodies (DLB) in a large PAF cohort with extended follow-up.

Methods: We performed a retrospective review of all patients with neurogenic orthostatic hypotension consistent with PAF from 1998-2008 seen at Mayo Clinic, Rochester. Patients were called to assess for development of motor, cognitive and autonomic symptoms and new diagnosis of MSA, PD or DLB. Clinical and laboratory variables before evolution were extracted and factors predictive of evolution assessed using multinomial logistical regression.

Results: Among 275 patients with PAF at presentation, $67(24 \%)$ phenoconverted to one of the synucleinopathies with motor involvement. Of those, $34(51 \%)$ met criteria for MSA while $33(49 \%)$ met criteria for PD or DLB. Median age of onset was younger in MSA phenoconverters (58.5 years; IQR 53-67) compared to stable PAF $(65.0 ; 56-72)$ and PD/DLB phenoconverters $(69.0 ; 64-75) \quad(\mathrm{p}$ $=0.0083)$. Clinical features at presentation influenced phenoconversion: severe bladder symptoms were more common in those evolving into MSA (41.2\%) compared to stable PAF $(7.7 \%)$ (p < 0.0001); subtle motor signs were more frequent in MSA (50.0\%) and PD/DLB phenoconverters $(27.3 \%)$ compared to stable PAF $(4.3 \%)(\mathrm{p}<0.0001)$. MSA phenoconverters were more likely to have supine norepinephrine levels $\geq 100 \mathrm{pg} / \mathrm{mL}$ (95.5\%) compared to stable PAF $(56.9 \%)$ and PD/DLB phenoconverters $(45.5 \%)(\mathrm{p}=0.0004)$. Preganglionic sweat loss was more common in MSA phenoconverters (43.8\%) compared to stable PAF (12.7\%) and PD/DLB phenoconverters $\quad(16.7 \%) \quad(\mathrm{p}=0.0004)$. Presentation variables predicting evolution to MSA included subtle motor signs, supine norepinephrine levels, severe bladder symptoms and dream enactment behavior. Presentation variables predictive of evolution to PD/DLB included: subtle motor signs, dream enactment behavior and bowel symptoms. Conclusions: Our findings suggest that at least one-quarter of PAF patients evolve into another synucleinopathy. Presentation features determine patients at risk for evolution with specific patterns indicative of phenoconversion to MSA versus PD/DLB.

Funding: Supported in part by NIH (P01NS44233, U54NS065736, K23NS075141, R01 FD004789, R01 NS092625) and Mayo CCaTS (UL1TR000135), and Cure PSP Foundation. Its contents are solely the responsibility of the authors and do not necessarily represent the official view of NIH.

\section{Cutaneous biomarkers in multiple system atrophy}

N. Wang $^{1}$, S. Rajan ${ }^{1}$, D. Kern ${ }^{2}$, J.A. Palma ${ }^{3}$, H. Kaufmann ${ }^{3}$, R. Freeman ${ }^{1}$, C.H. Gibbons ${ }^{1}$

${ }^{1}$ Department of Neurology, Beth Israel Deaconess Medical Center, Harvard Medical School, Boston, MA, USA; ${ }^{2}$ University of Colorado, Denver, CO, USA; ${ }^{3} \mathrm{New}$ York University, New York, NY, USA

Background: The deposition of phosphorylated alpha-synuclein in both MSA and PD has created challenges in differentiating the synucleinopathies.

Objective: To identify an objective marker to distinguish MSA from PD.

Methods: Twenty-one individuals with MSA, 54 individuals with PD and 20 healthy control subjects had skin biopsies taken from multiple proximal and distal sites. Skin biopsies were stained for multiple neural markers including PGP 9.5, phosphorylated alpha-synuclein and tyrosine hydroxylase (TH).

Results: Phosphorylated alpha-synuclein was detected in all patients with PD and MSA, but not in any control subjects $(\mathrm{P}<0.001,100 \%$ sensitivity and specificity for MSA and PD compared to controls). The ratio of TH to PGP 9.5 in sudomotor fibers was significantly increased in sweat glands from subjects with MSA compared to controls $(\mathrm{P}<0.001)$ and to individuals with $\mathrm{PD}(\mathrm{P}<0.001)$. The pilomotor nerve fiber density (PMNFD) was decreased in individuals with PD compared to MSA or controls (P93\% sensitivity and specificity in distinguishing PD from MSA).

Discussion: Skin biopsy, in combination with a series of different immunostains, can provide significant value as a comprehensive diagnostic tool. Subjects with synucleinopathies can be identified by the presence of phosphorylated alpha-synuclein, which is not present in control subjects. Evidence of diminished PMNFD and a normal sudomotor TH ratio strongly suggests a diagnosis of PD, while normal PMNFD and increased sudomotor TH ratios supports a diagnosis of MSA. The shift towards sympathetic sudomotor adrenergic innervation within sweat glands in MSA may suggest ongoing sympathetic cholinergic damage and repair, a finding seen previously in rodent models. The clinical significance of this finding is unknown, and requires confirmation, but suggests a potential biomarker to differentiate PD from MSA.

Funding: Study supported by the MSA Foundation (RF) and NIH U54 NS065736. 
Long-term administration of intrathecal mesenchymal stem cells in multiple system atrophy - a compassionate use experience

W. Singer, A.B. Dietz, A.D. Zeller, T.L. Gehrking, J.D. Schmelzer, D.M. Sletten, J.A. Gehrking, A.M. Schmeichel, E.A. Coon,

P. Sandroni, E.E. Benarroch, J.Y. Matsumoto, J.H. Bower, A. Hassan, A. McKeon, P.A. Low

Department of Neurology, Mayo Clinic, Rochester, MN, USA

Objective: To explore the long-term safety of repeated intrathecal administration of autologous mesenchymal stem cells (MSCs) as therapeutic approach to multiple system atrophy (MSA).

Background: We recently completed a phase I/II dose-escalation study of autologous, adipose-derived MSCs in MSA. The study showed an adequate safety-profile and treatments were well tolerated up to a dose of 50 million cells per injection. There were compelling clinical and biomarker efficacy signals supporting continued exploration of this approach.

Methods: Patients who completed the original phase I/II study were invited to receive additional intrathecal MSC administrations of 50 million cells per injection every 6 months. Patients were followed with serial clinical, laboratory, and MRI surveillance. Primary endpoints were frequency and type of adverse events (AEs); secondary endpoints included clinical measures of disease progression (Unified MSA Rating Scale, UMSARS).

Results: Of 16 patients who met in- and exclusion criteria all chose to participate. The extension protocol has been ongoing for over 4 years, up to 8 additional injections have been given per patient, and over 60 extension injections administered in total. At this time, one patient remains active, 8 patients have passed away, and the other patients have dropped out due to advanced disease stage, travel hardship, or loss of perceived benefit. The repeated injections have shown a similarly favorable AE profile as the original trial. Main AEs continue to be abnormalities on MRI imaging of the cauda equina and selflimited low back discomfort, in none of the patients troublesome enough to decline further treatments. The rate of disease progression remained slower than observed in the placebo cohort of the Rifampicin trial, but was faster than in the main MSC study.

Conclusions: Repeated, long-term intrathecal MSC administration in MSA is safe and well tolerated. The efficacy signal seems to become less robust as the disease progresses.

Funding: Supported by NIH (P01NS44233, U54NS065736, K23NS075141, R01NS092625, UL1TR000135), FDA (R01FD004 789), Cure MSA Foundation, and Mayo Funds.
Autonomic changes in Parkinson's disease patients treated with L-threo-3,4-dihydroxyphenylserine and high dose Carbidopa therapy

K.R. Hay, K.E. McDonell, P. Trujillo, C. Shibao, S. Paranjape, O. Roman, D.O. Claassen

Department of Neurology, Vanderbilt University Medical Center, Nashville, TN, USA

Low levels of peripheral norepinephrine (NE) are noted in Parkinson's disease (PD) patients and relate to autonomic disability. This open label dose-escalation safety and tolerability study sought to evaluate if inhibition of peripheral dopa decarboxylase (DDC) using high doses of Carbidopa therapy prevented peripheral breakdown of L-threo-3,4-dihydroxyphenylserine (L-DOPS). Participants ( $\mathrm{n}=15$, 9/6 female/male, $70 \pm 10$ ) were assessed at baseline, Carbidopa (200 mg BID), and at maximum tolerated dose of Carbidopa and L-DOPS (up to $600 \mathrm{mg} \mathrm{BID)}$ ). The duration of the assessment was 11 weeks. 8 completed autonomic function testing which included standard autonomic function testing (i.e., tilt-table assessment which included Valsalva maneuver), and peripheral catecholamine measurement (NE levels, and dihydroxyphenylglycol (DHPG)). During the Valsalva maneuver, we calculated the difference in systolic blood pressure (SBP) between phase 4 and the baseline (delta SBP $=$ SBPphase 4 SBPbaseline) as a marker of autonomic function. Wilcoxon signed rank tests were used to assess treatment differences, with two-sided defined significance $p<0.05$. Results indicated there was no change in plasma NE levels $(\mathrm{p}=0.6)$, DHPG $(\mathrm{p}=0.3)$, or delta SBP $(\mathrm{p}=0.6)$ from baseline to the treatment visit. At the Carbidopa visit, $25 \%$ of patients experienced lower blood pressure at 3 minutes upright during the tilt table test. These results suggest that high doses of Carbidopa prevent peripheral breakdown of L-DOPS. Further research is needed to evaluate the central effects of NE on autonomic function.

Funding: American Academy of Neurology Clinical Research Training Fellowship M0042136 to KM, Lundbeck 54580 to KM, VICTR VR17015 to KM, faculty advisor DC. 


\section{POSTER PRESENTATIONS \\ Wednesday, November 6, 2019}

\section{POSTER SESSION I}

\section{Poster \#1} Addressing orthostatic hypotension in Parkinson disease patients
in a neurology resident continuity clinic: one year follow-up

E.P. Golden ${ }^{1}$, T. Le ${ }^{1}$, B. Bleiberg ${ }^{1}$, L.S. Brown ${ }^{2}$, S. Khan ${ }^{1}, \mathrm{~S}$.

Vernino $^{1}$, P. Khemani ${ }^{3}$

${ }^{1}$ University of Texas Southwestern Medical Center, Dallas, TX, USA;

${ }^{2}$ Parkland Health and Hospital System, Dallas, TX, USA; ${ }^{3}$ Swedish

Neuroscience Institute, Seattle, WA, USA

Objective: This quality improvement project aims to improve the frequency of orthostatic hypotension $(\mathrm{OH})$ assessment and treatment in Parkinson disease (PD) patients managed at Parkland neurology resident clinic, a safety-net clinic.

Background: $\mathrm{OH}$ is prevalent in PD and associated with troublesome symptoms and adverse outcomes. Although routine screening is recommended in PD, it is often neglected.

Methods: This project follows the "Plan-Do-Study-Act" method. Baseline data were collected from fiscal year (FY) 2016. Two areas of unmet need were identified: 1) asking patients about orthostatic symptoms (56\% of patients), and 2) measuring orthostatic vital signs (15\%). An intervention was then developed and implemented. This included tailored education of faculty, residents, and clinic staff, a workflow change to measure orthostatic vital signs (VS) in all PD patients, and the addition of "smartphrases" in the electronic medical record to assist providers with the documentation and management of OH. Chart review of PD patients in FY 2018 was conducted for follow up data, including details of $\mathrm{OH}$ evaluation: symptom documentation, orthostatic VS measurement, and $\mathrm{OH}$ management.

Results: 162 patients were identified. The population did not differ significantly from baseline in age, ethnicity, language, disease duration, or number of clinic visits in the past year. $69 \%$ of patients were queried about $\mathrm{OH}$ symptoms (improved from $56 \%, \mathrm{p}=0.03$ ), $38 \%$ of whom endorsed symptoms. $49 \%$ had orthostatic VS measured (improved from $15 \%, \mathrm{p}<0.01)$. $11 \%$ of the population was diagnosed with $\mathrm{OH}$ (increased from $2 \%, \mathrm{p}<0.01$ ), and $12 \%$ had orthostatic symptoms managed pharmacologically or non-pharmacologically (improved from $2 \%, \mathrm{p}<0.01$ ).

Conclusions: A multifaceted intervention including education and workflow adjustment was effective in improving rates of querying PD patients about $\mathrm{OH}$ symptoms and measuring orthostatic VS in a safety-net clinic. These data can be used to develop additional interventions to further increase attention to this important complication of PD.

\section{Poster \#2}

Disease stage and UMSARS progression in patients with multiple system atrophy enrolled in the Natural History Study of the Synucleinopathies: implications for clinical trials

M.A. Perez ${ }^{1}$, J.-A. Palma ${ }^{1}$, L. Norcliffe-Kaufmann ${ }^{1}$, W. Singer ${ }^{2}$, P. Low $^{2}$, M.T. Pellecchia ${ }^{3}$, H.-J. Kim ${ }^{4}$, C. Shibao ${ }^{5}$, A. Peltier ${ }^{5}$, I.
Biaggioni $^{5}$, D. Giraldo ${ }^{6}$, M.J. Marti ${ }^{6}$, A. Fanciulli ${ }^{7}$, C. Terroba ${ }^{8}$, M. Merello $^{8}$, D.S. Goldstein ${ }^{9}$, R. Freeman ${ }^{10}$, C.H. Gibbons ${ }^{10}$, S. Vernino $^{11}$, F. Krismer ${ }^{7}$, G. Wenning ${ }^{7}$, H. Kaufmann ${ }^{1}$; on behalf of the Natural History Study of the Synucleinopathies Study Group ${ }^{1}$ Department of Neurology, New York University School of Medicine, New York, NY, USA; ${ }^{2}$ Department of Neurology, Mayo Clinic, Rochester, MN, USA; ${ }^{3}$ Department of Neurology, Salerno University, Salerno, Italy; ${ }^{4}$ Department of Neurology, Seoul National University, Seoul, South Korea; ${ }^{5}$ Department of Medicine and Pharmacology, Vanderbilt University, Nashville, TN, USA;

${ }^{6}$ Movement Disorders Unit, Neurology Service, Hospital Clínic de Barcelona, Institute of Neuroscience, University of Barcelona, Barcelona, Spain; ${ }^{7}$ Department of Neurology, Innsbruck Medical University, Innsbruck, Austria; ${ }^{8}$ Movement Disorders Division, FLENI, Buenos Aires, Argentina; ${ }^{9}$ Neurocardiology Section, Intramural NINDS, Bethesda, MD, USA; ${ }^{10}$ Department of Neurology, Beth Israel Deaconess Medical Center, Boston, MA, USA;

${ }^{11}$ Department of Neurology, University of Texas Southwestern, Dallas, TX, USA

Background: Disease progression of multiple system atrophy (MSA) as measured by the Unified Multiple System Atrophy Rating Scale (UMSARS) varied significantly in natural history studies. Reported 1-year UMSARS-1 and UMSARS-2 progression rates ranged from 3.9 to 6.5 and 3.5 to 8.2 , respectively. We hypothesize that this variability is due, at least in part, to differences in severity at enrollment and a potential ceiling effect in the scale, so that patients in more advanced stages may appear to worsen less, which would have important implications for clinical trial design.

Methods: We analyzed the rate of change in the UMSARS in a large international cohort of well-characterized patients with a clinical diagnosis of possible or probable MSA enrolled in the Natural History Study of Synucleinopathies. Annualized progression rates were obtained using 2-year follow-up data.

Results: 293 patients $(62.0 \pm 7.8$ years old) with MSA were enrolled. Disease duration was $4.5 \pm 3.6$ years. 98 patients completed 1-year evaluations and 48 completed the 2-year evaluation. The 12-month progression rates were $5.5 \pm 5.3$ for the UMSARS-I, $6.6 \pm 5.2$ for the UMSARS-II, and $11.9 \pm 9.8$ for the total score. The 24-month progression rates were $10.8 \pm 7.1$ for the UMSARS-I, $12.5 \pm 7.9$ for the UMSARS-II, and $22.6 \pm 13.7$ for the total score. Annualized progression rates were divided according to their baseline UMSARS-I and UMSARS II. There was a significant $(\mathrm{p}=0.0461)$ inverse relationship between rate of progression and UMSARS-I at baseline. A similar, but not significant trend was observed with UMSARS-II at baseline.

Conclusions: The rate of progression as measured by UMSARS is influenced by the baseline disease severity. A ceiling effect should be considered when planning enrollment, power calculations, and outcome measures in clinical trials.

Funding: MSA Coalition and NIH (U54-NS065736-01).

\section{Poster \#3}

\section{Symptoms of orthostatic hypotension}

S. Rajan, J. Celli, I. Bonyhay, C.H. Gibbons, R. Freeman Department of Neurology, Beth Israel Deaconess Medical Center, Boston, MA, USA

Background: Many patients with orthostatic hypotension $(\mathrm{OH})$ are asymptomatic and do not endorse several symptoms of $\mathrm{OH}$ on 
validated questionnaires. Additionally, symptom severity scores correlate poorly with tilt BP and the BP fall.

Objective: To improve reporting of symptoms of orthostatic hypotension.

Methods: After tilt testing, patients were asked to (1) spontaneously report and rate their symptoms without investigator guidance; (2) select and rate symptoms from a symptom list with investigator guidance and subsequent rating training; and (3) report and rate their symptoms during activities of daily life (ADL).

Results: Eighteen patients with $\mathrm{OH}$ were studied (mean age 73 \pm 7.3 ). Tilted SBP was $94 \pm 24.1 \mathrm{mmHg}$ with a SBP fall of $62 \pm 25.1 \mathrm{mmHg}$. Spontaneously reported symptoms during tilt included lightheadedness $(30.4 \%)$, dizziness $(7.7 \%)$, weakness $(7.7 \%)$ and tingling $(7.7 \%)$. Mean symptom rating was $4.7 \pm 2.1 / 10$. Selecting symptoms from a list with investigator guidance after tilt, symptom prevalence was: lightheadedness (72.2\%), dizziness $(50 \%)$, weakness $(33.3 \%)$, flushing $(50.0 \%)$, leg pain, leg weakness and tiredness (all 33.3\%), and tingling (16.7\%). Symptoms occurring during ADL were lightheadedness $(77.8 \%)$, dizziness $(72.2 \%)$, weakness $(66.7 \%)$, feeling of impending black out $(66.7 \%)$, aching in the back of neck and shoulders $(66.7 \%)$, fatigue, tiredness, backache, general discomfort (all $61.1 \%$ ) and headache (55.6\%). Symptom scores correlated poorly with tilted BP and fall in BP. With investigator-guided rating training, six patients adjusted their spontaneous symptom severity rating by 2 or more points.

Discussion: Patients with $\mathrm{OH}$ report few symptoms and symptoms do not correlate with tilt BP and BP fall. When given a symptom list, more symptoms of $\mathrm{OH}$ are reported than reported spontaneously. $\mathrm{OH}$ symptom scores tend to change with investigator training. Symptoms occurring during tilt do not fully capture $\mathrm{OH}$ symptoms during daily life. These data suggest that $\mathrm{OH}$ assessment in clinical practice and clinical trials can be improved.

Funding: Theravance.

\section{Poster \#4}

\section{Conjugal multiple system atrophy: chance, shared risk factors, or evidence of infectivity?}

\author{
E.A. Coon, W. Rocca, J.E. Ahlskog, J.Y. Matsumoto, P.A. Low, \\ W. Singer \\ Department of Neurology, Mayo Clinic, Rochester, MN, USA
}

Objective: To describe conjugal multiple system atrophy (MSA) in a couple married for 44 years and report environmental risk factors which may contribute to the occurrence.

Methods: Case description of conjugal MSA with report of shared environmental risk factors and retrospective review of all MSA patients diagnosed between 1998-2012 with an autonomic reflex screen. Probability calculation was based on the age specific point prevalence of MSA.

Results: A husband and wife both developed MSA symptoms at age 63. The husband's onset was of imbalance, followed by falls and genitourinary failure; parkinsonism and antecollis was evident on examination. Autonomic function testing showed widespread autonomic failure with composite autonomic severity score (CASS) of 9 and predominantly central anhidrosis on thermoregulatory sweat test. The patient died 2.25 years after symptom onset. The wife initially developed urinary symptoms progressing to incontinence. Parkinsonism, dysphonia and falls began within 1 year and she underwent tracheostomy for stridor. Autonomic function testing showed severe autonomic failure with composite autonomic severity score (CASS) of 9 and predominantly central anhidrosis on thermoregulatory sweat test. Interview with the surviving patient and son revealed substantial pesticide and chemical exposure. Upon review of 685 consecutively diagnosed MSA patients, there were no other cases of conjugal MSA. Assuming point prevalence of MSA based on population studies and independence of the two events, the probability of both individuals developing MSA is 6.08 e-9.

Conclusion: Based on the point prevalence of MSA in the population, conjugal MSA is extremely rare but possible. Conjugal MSA may occur by chance; however, exposure to shared risk factors may contribute to the occurrence. As this is the first reported case of conjugal MSA to our best knowledge, evidence for infectivity between spouses is lacking.

Funding: Supported in part by NIH (P01NS44233, U54NS065736, K23NS075141, R01 FD004789, R01 NS092625) and Mayo CCaTS (UL1TR000135), and Cure PSP Foundation. Its contents are solely the responsibility of the authors and do not necessarily represent the official view of NIH.

\section{Poster \#5}

Long-term trends in myocardial sympathetic innervation and function in synucleinopathies

\section{G. Lamotte $^{I}$, C. Holmes ${ }^{2}$, T. $\mathrm{Wu}^{3}$, D.S. Goldstein ${ }^{4}$}

${ }^{1}$ Clinical Neurosciences Program (CNP), Division of Intramural Research, National Institute of Neurological Disorders and Stroke (NINDS), Bethesda, MD, USA; ${ }^{2}$ Department of Neurology, Medstar Georgetown University Hospital, Washington, DC, USA; ${ }^{3}$ Clinical Neurocardiology Section, CNP/DIR/NINDS/NIH, Bethesda, MD, USA; ${ }^{4}$ Clinical Trials Unit, NINDS, Bethesda, MD, USA

Background: Parkinson disease (PD), pure autonomic failure (PAF), and multiple system atrophy (MSA) are characterized by intra-cerebral deposition of the protein alpha-synuclein and are termed synucleinopathies. Lewy body synucleinopathies involve decreased cardiac sympathetic innervation and functional abnormalities in residual noradrenergic terminals. This observational, retrospective, cohort study describes long-term trends in indices of cardiac sympathetic innervation and function in synucleinopathies.

Methods: Patients with PD ( $=31,12$ with orthostatic hypotension $(\mathrm{PD}+\mathrm{OH}))$, PAF $(\mathrm{N}=9)$, or MSA $(\mathrm{N}=9)$ underwent repeated $18 \mathrm{~F}$ dopamine positron emission tomography (median follow-up 3.5 years). Interventricular septal 18F-dopamine-derived radioactivity 8 minutes after tracer injection ( 8 ' Radioactivity) was used as an index of sympathetic innervation and the slope of mono-exponential decline of radioactivity between 8 and 25 minutes $\left(\mathrm{k}^{\prime}-25^{\prime}\right)$ as an index of intraneuronal vesicular storage. Healthy volunteers ( $\mathrm{HVs})(\mathrm{N}=33)$ and individuals at high risk of $\mathrm{PD}(\mathrm{N}=15)$ were controls.

Results: Upon initial evaluation the groups with $\mathrm{PD}+\mathrm{OH}, \mathrm{PAF}$, or $\mathrm{PD}$ no $\mathrm{OH}$ had low mean 8 ' radioactivity compared to HVs $(\mathrm{p}<0.0001$, $\mathrm{p}=0.0002, \mathrm{p}=0.006)$ and had elevated $\mathrm{k} 8^{\prime}-25^{\prime}(\mathrm{p}=0.0007, \mathrm{p}=0.007$, $\mathrm{p}=0.06)$. There was no significant difference between MSA and HVs. 8 ' radioactivity decreased by a median of $4 \%$ per year in PD (3\% in $\mathrm{PD}+\mathrm{OH}, 4.1 \%$ in PD without $\mathrm{OH}$ ) and did not decrease in MSA. k8'25 ' values did not change during follow-up in any group.

Conclusions: Neuroimaging evidence of decreased vesicular uptake in cardiac sympathetic nerves is present upon initial evaluation of patients with Lewy body synucleinopathies and may provide a biomarker of catecholaminergic dysfunction early in the disease process. Funding: This research was supported by the Division of Intramural Research of the National Institute of Neurological Disorders and Stroke (NINDS), NIH. 


\section{Poster \#6}

\section{Reliable indicators of autonomic function test in Parkinson's disease}

\section{B.A. Yoon, J.K. Kim, J.W. Kim, S.M. Cheon}

Department of Neurology, Dong-A University Hospital, Busan, South Korea

Introduction: Nonmotor symptoms of Parkinson's disease (PD) are known to play an important role in daily activity and autonomic function is one of representative symptoms. However, it is rarely known which of the indicators of various autonomic function tests (AFT) are associated with clinical features. Therefore, we tried to identify the indicators using well organized autonomic function tests, modified composite autonomic severity scores (CASS).

Methods: Patients were consecutively recruited from the out-patient clinic of a single tertiary hospital since March 2015. In total, 124 patients with PD were enrolled and constructed quantitative sudomotor axonal reflex (QSART), heart rate response to deep breathing (HRdB), Valsalva maneuver, and head up tilt (HUT) test. And the results were compared with various clinical features. Patients were divided into normal and abnormal groups, each according to variable indicators composed of CASS, such as E:I ratio, HRdB, Valsalva ratio (VR), pressure recovery time (PRT), presence of orthostatic hypotension $(\mathrm{OH})$ and CASS score.

Results: HRdB was correlated with gastrointestinal, sex scores of autonomic dysfunction questionnaire (ADQ) and gastrointestinal, cardiovascular scores of Non-Motor Symptoms Scale (NMSS). VR abnormality was related with new activities of daily living questionnaire and gastrointestinal score of NMSS. On the other hand, PRT and presence of $\mathrm{OH}$ was related to cardiovascular, gastrointestinal and urinary score of ADQ and gastrointestinal score of NMSS. Concurrent validity was shown as a significant positive correlation between the prolonged PRT and motor and activities of daily living scores of Korean version of Parkinson's Disease Quality of Life 39.

Conclusion: We suggest HRdB, VR, prolonged PRT and presence of $\mathrm{OH}$ would be candidate of indicators of non motor symptoms in PD.

\section{Poster \#7}

Post-mortem and in vivo evidence for "sick but not dead" central dopaminergic neurons in multiple system atrophy: implications for neurotrophic factor gene enhancement therapy

\section{D.S. Goldstein, P. Sullivan, C. Holmes, K. Bankiewicz}

Autonomic Medicine Section (formerly Clinical Neurocardiology Section), CNP/DIR/NINDS/NIH, Bethesda, MD, USA

Background: Multiple system atrophy (MSA) features autonomic failure, parkinsonism or cerebellar ataxia, and glial cytoplasmic inclusions (GCIs) that contain an abundance of the protein alphasynuclein (AS). The occurrence of GCIs in MSA suggests the disease involves derangements of glial neurotrophic functions. Glial cell linederived neurotrophic factor (GDNF) is absolutely required for maintaining adult catecholaminergic neurons, putamen dopamine content is drastically decreased in MSA, and cerebrocortical GDNF is decreased in MSA. These findings suggest that intra-putaminal administration of adeno-associated virus (AAV)-GDNF by MRIguided convection-enhanced delivery might slow the neurodegenerative process. Such treatment would be rational if there were residual neurons upon which GDNF could act. We explored whether MSA entails functional abnormalities in residual central dopaminergic neurons-the "sick but not dead" phenomenon.
Methods: Denervation alone would be expected to decrease levels of catecholamines and their metabolites equivalently. Post-mortem putamen tissue contents of catechols and catechol ratios were used as indices of synthesis, vesicular storage, and metabolism of catecholamines in controls and MSA patients. In vivo indices of dopaminergic intra-neuronal functions included the washout of putamen 18F-DOPA-derived radioactivity in the putamen and catechol ratios in cerebrospinal fluid (CSF).

Results: Compared to controls, MSA patients had decreased mean putamen dopamine (by 96\%, p<0.0001) and GDNF (by 76\%, $\mathrm{p}=0.0042$ ). Indices of putamen L-aromatic-amino-acid decarboxylase, vesicular storage, and aldehyde dehydrogenase (ALDH) were all decreased $(p<0.0001, p=0.0042, p=0.039)$. Putamen washout of $18 \mathrm{~F}$ DOPA-derived radioactivity was accelerated $(\mathrm{p}<0.0001)$, and CSF 5-S-cysteinyldopamine/3,4-dihydroxyphenylacetic acid, an index of spontaneous vs. enzymatic oxidation of cytoplasmic dopamine and an inverse index of ALDH activity, was increased ( $\mathrm{p}=0.0050)$.

Conclusions: In MSA there is a population of putamen dopaminergic neurons that are dysfunctional but extant. The results support the feasibility of AAV-GDNF reversing GDNF deficiency, enabling "sick but not dead" residual neurons to sprout and thereby slowing or reversing the progression of monoaminergic neurodegeneration. Funding: Division of Intramural Research, NINDS, NIH.

\section{Poster \#8}

Longitudinal changes in autonomic symptom burden predict decline in activities of daily living in Parkinson's disease

M. Sklerov ${ }^{1}$, C. Shih ${ }^{3}$, N. Browner ${ }^{1}$, J.-A. Palma ${ }^{4}$, E. Dayan ${ }^{2,3}$ Departments of ${ }^{1}$ Neurology and ${ }^{2}$ Radiology, University of North Carolina, Chapel Hill, NC, USA; ${ }^{3}$ Biomedical Research Imaging Center, University of North Carolina, Chapel Hill, NC, USA; ${ }^{4}$ Department of Neurology, Dysautonomia Center, New York University School of Medicine, New York, NY, USA

Introduction: Autonomic dysfunction in Parkinson's disease (PD) is common and a major determinant of poor outcomes. The primary aim of this study was to examine the relationship of longitudinal changes in autonomic symptom burden and longitudinal changes in activity of daily living (ADL) function.

Methods: Data from the Parkinson's Progression Markers Initiative (PPMI), a natural history database of patients with newly diagnosed (within 2 years) PD, were used for this analysis. Datapoints from visit 6 (24 months after enrollment), and visit 12 (60 months after enrollment) were investigated, along with baseline data. We examined the impact of longitudinal changes in the Scales for Outcomes in Parkinson's disease - Autonomic (SCOPA-AUT) on longitudinal changes in the Schwab and England ADL function scale. As a secondary analysis, we performed a mediation analysis to investigate whether longitudinal changes in symptoms of depression, as measured with the Geriatric Depression Scale (GDS), mediate the relationship between longitudinal changes in autonomic symptom burden and ADL function.

Results: Though changes in autonomic symptom burden, cognitive function, depression, and motor function all correlated with ADL function, only changes in ADL function and depression were associated with changes in autonomic symptom burden. We found that longitudinal change in autonomic symptoms was a significant predictor of change in ADL function at 24 months and 60 months, with the cardiovascular subscore proving to be a major driver of this association. Mediation analysis revealed that the association between autonomic symptoms and ADL function is partially mediated by depression severity. 
Conclusions: Longitudinal changes in autonomic symptoms appear to impact ADL function in early PD, both directly and indirectly through their impact on depressive symptoms. Future investigation is warranted into the impact of treatment of autonomic symptoms and depressive symptoms on outcomes of ADL function and quality of life measures in PD.

Funding: The project described was supported by the National Center for Advancing Translational Sciences (NCATS), National Institutes of Health, through Grant Award Number UL1TR002489. The content is solely the responsibility of the authors and does not necessarily represent the official views of the NIH (Dr. Sklerov), R01AG062590 (NIH) (Dr. Dayan).

\section{Poster \#9}

\section{Submandibular gland alpha-synuclein deposition occurs without local sympathetic denervation or dysfunction in Parkinson disease}

\section{R. Isonaka, P. Sullivan, D.S. Goldstein}

Autonomic Medicine Section (formerly Clinical Neurocardiology Section), CNP/DIR/NINDS/NIH, Bethesda, MD, USA

Background: The neurochemical hallmark of Parkinson disease (PD) is dopamine deficiency in the nigrostriatal system, and a neuropathologic hallmark is Lewy bodies that contain alpha-synuclein (AS). Genetic studies have implicated AS in the striatal dopaminergic lesion attending rare familial forms of PD, but whether AS deposition is neurotoxic to catecholaminergic neurons in general in PD has been unclear. AS is found in sympathetically innervated structures such as submandibular salivary glands. We examined whether AS colocalization with tyrosine hydroxylase (TH), a marker of sympathetic noradrenergic innervation, in submandibular gland sympathetic nerves is related to biochemical indices of local sympathetic innervation and function in $\mathrm{PD}$.

Methods: Coded post-mortem submandibular gland tissue samples from 7 control subjects and 8 PD patients were assayed for AS, TH, and smooth muscle actin (SMA) by quantitative immunofluorescence confocal microscopy. Submandibular gland and apical myocardial catechols in the same subjects were assayed by batch alumina extraction followed by liquid chromatography with electrochemical detection. The groups were compared by independent-means t-tests conducted on log-transformed data.

Results: In submandibular gland tissue the PD group had higher ASTH colocalization indices, higher AS levels, and higher AS/SMA and AS/TH ratios than did the controls $(\mathrm{p}=0.00021,0.0023,0.0015$, 0.00073 ), with no group differences in $\mathrm{TH}$, catecholamines, or catechol ratios. Myocardial tissue norepinephrine in PD patients was markedly decreased (by 99\%, p<0.0001), associated with abnormal catechol ratios indicating deficiencies in catecholamine synthesis and vesicular storage in residual nerves.

Conclusions: PD involves AS buildup in sympathetic noradrenergic nerves in submandibular gland tissue. This buildup is unrelated to local noradrenergic innervation or function, whereas in the same patients there are striking sympathetic abnormalities in the heart. Intra-neuronal AS deposition alone seems insufficient to explain the myocardial noradrenergic deficiency attending PD. Analogously, such deposition alone might also be insufficient to explain nigrostriatal dopamine depletion in PD.

Funding: Division of Intramural Research, NINDS, NIH.

\section{Poster \#10}

Colocalization of alpha-synuclein with tyrosine hydroxylase in skin biopsies from individuals at genetic risk of Parkinson disease: a genotype-phenotype study

R. Isonaka, D. Narendra, D.S. Goldstein

Autonomic Medicine Section (formerly Clinical Neurocardiology Section) CNP/DIR/NINDS/NIH, Bethesda, MD, USA

Background: A neuropathologic hallmark of sporadic Parkinson disease (PD) is Lewy bodies, which contain an abundance of alphasynuclein (AS). AS genotypic abnormalities cause rare familial forms of PD. A53T mutation of the gene encoding AS (SNCA, PARK1), replication of the normal SNCA gene (PARK4), and glucocerebrosidase GBA mutation can involve neurogenic orthostatic hypotension and cardiac norepinephrine depletion, indicating a pathogenetic role of AS in sympathetic noradrenergic deficiency. We report results from an ongoing collaborative genotype-phenotype case series about AS-TH colocalization in skin biopsies from rare individuals with genotypic abnormalities known to cause familial PD. PARK1, PARK4, PARK7 (from DJ-1 mutation), and GBA mutation are thought to be associated with brain Lewy bodies in most cases, whereas PARK2 (from parkin mutation) is not. Whether these differences obtain based on analyses of sympathetic noradrenergically innervated skin constituents in living patients has been unknown.

Methods: Arrector pili muscles, which receive purely sympathetic noradrenergic innervation, were analyzed by immunofluorescence confocal microscopy for AS, tyrosine hydroxylase (TH, a marker of sympathetic noradrenergic nerves), and smooth muscle actin (SMA). The extent of AS-TH colocalization was calculated using a previously validated colocalization index in subjects with PARK1 $(\mathrm{N}=1)$, PARK2 $(\mathrm{N}=2)$, PARK4 $(\mathrm{N}=1)$, PARK7 $(\mathrm{N}=1)$, and GBA mutation $(\mathrm{N}=1)$.

Results: AS-TH colocalization indices in PARK1, PARK4, PARK7, and GBA mutation were increased from values in an ongoing database of controls. AS-TH colocalization indices were not increased in PARK2.

Conclusions: PARK1, PARK4, PARK7, and GBA mutation involve AS in sympathetic noradrenergic nerves; so far in this case series PARK2 does not. This pattern fits well with post-mortem neuropathological data about the occurrence of central nervous system Lewy bodies in these conditions. The results support the utility of ASTH colocalization as a means to diagnose synucleinopathy in living persons at genetic risk of PD.

Funding: Division of Intramural Research, NINDS, NIH.

\section{Poster \#11}

\section{A case of indifference to pain and central anhidrosis}

K. Shouman, K.V. Minota Pacheco, A.M. Schmeichel, T.L. Gehrking, J.A. Gehrking, A.D. Zeller, P.A. Low, W. Singer

Division of Autonomic Disorders, Department of Neurology, Mayo Clinic, Rochester, MN, USA

Objective: We describe an unusual case of congenital indifference to pain with preganglionic sudomotor dysfunction.

Background: Pain insensitivity and anhidrosis are well-recognized features of hereditary sensory and autonomic neuropathy (HSAN), type IV, due to a NTRK1 gene mutation. We report a case of indifference to pain and anhidrosis without evidence of peripheral sensory or autonomic neuropathy. 
Case report: A 19-year-old woman with history of mild intellectual disability presented to our clinic with suspicion for HSAN IV. She had a lifelong history of inability to sense pain which had led to multiple mechanical and thermal injuries, as well as neurotrophic cornea. In spite of her insensitivity to pain, however, medical procedures known to be associated with pain could induce behavioral spells. Her inability to sweat resulted in heat intolerance and episodes of heat exhaustion with flushing and lethargy. Other associated symptoms included decreased ability to sense hunger and bladder fullness. She had no self-mutilating behavior and no sicca symptoms. Her neurological examination revealed mild disinhibition, intellectual impairment and indifference to pain whether generated by cooling, heat, or pinprick. Exam was otherwise normal including other sensory modalities. Testing including brain MRI, EEG, EMG, autonomic reflex screen (including QSART), orthostatic catecholamines, epidermal and sweat gland nerve fiber density, and sural nerve biopsy (including electron microscopy to assess small fibers) were normal. Thermoregulatory sweat test revealed global anhidrosis indicating preganglionic sudomotor failure. Genetic testing demonstrated no mutation of the NTRK1 gene but did reveal a heterozygous variant of unknown significance of SPG11, p.S1142C (Ser1142Cys), c.3425C $>$ G, Chr15:44900670. Whole exome sequencing (WES) is currently pending.

Conclusions: This case demonstrates a very intriguing combination of indifference to pain and certain internal stimuli including hunger with central anhidrosis. It emphasizes the close relationship between central pain processing and the central autonomic network.

\section{Poster \#12}

Neurogenic bladder dysfunction in patients with familial dysautonomia: a cross-sectional study

\author{
B.J. Balgobin ${ }^{1}$, J.-A. Palma ${ }^{1}$, B. Peyronnet ${ }^{2}$, B. Brucker ${ }^{2}$, \\ H. Kaufmann ${ }^{1}$ \\ ${ }^{1}$ Department of Neurology, Dysautonomia Center, New York \\ University School of Medicine, New York, NY, USA; ${ }^{2}$ Department of \\ Urology, New York University School of Medicine, New York, NY, \\ USA
}

Objective: To determine the frequency and symptomatic burden of neurogenic lower urinary tract dysfunction in patients with familial dysautonomia (FD).

Background: FD is a rare genetic disease caused by a founder mutation in the IKBKAP gene characterized by impaired development of sensory and afferent autonomic nerves. Some patients with FD complain of urinary symptoms but there are no reports on their frequency or characteristics.

Methods: We studied consecutive patients with FD between October 2017 and September 2018. All patients underwent urinalysis and ultrasound bladder post-void-residual (PVR) volume measurement; and completed the following symptom scales: International Prostate Symptom Score (IPSS), International Consultation on Incontinence Questionnaire on Urinary Incontinence-Short-Form (ICIQ-UI-SF), and Qualiveen-SF. We also performed a chart review to document renal ultrasound and urinary tract infections (UTI) within the past 5 years. To define medications anticholinergic and sedative effect we used the Anticholinergic Drug Scale (ADS). The Brief Ataxia Rating Scale (BARS) was used to evaluate motor disability.

Results: We studied 45 patients (22 men, 23 women, 24.3 \pm 13.1 years). Mean PVR was $54.2 \pm 13.1 \mathrm{ml}$. Five patients $(11.6 \%)$ had a
PVR $>100 \mathrm{ml}$ indicating underactive bladder. Patients had a low burden of lower urinary tract symptoms [IPSS: $4.5 \pm 4.1$ (out of 35); ICIQ-UI-SF: $1.9 \pm 3$ (out of 21), Qualiveen-SF: $0.3 \pm 0.3$ (out of 32)]. The PVR was not associated with symptom scores. Of the 15 patients who had renal ultrasound available, 1 had mild hydronephrosis. Urinary retention was not associated with frequency of UTI. Patients with urinary retention had higher anticholinergic side effect burden (ADS score: 4.2 vs. $1.3 ; \mathrm{P}=0.0179$ ) and worse ataxia (BARS score: 19.8 vs. $13.2, \mathrm{P}=0.0272$ ) than those without retention.

Conclusion: Neurogenic detrusor underactivity was present in $15 \%$ of patients with FD. Importantly, patients with detrusor underactivity were more frequently receiving anticholinergic medications and had greater disease-related motor disability. This has therapeutic implications.

Funding: Familial Dysautonomia Foundation.

\section{Poster \#13}

\section{Frequency of seizures and epilepsy in familial dysautonomia}

B.J. Balgobin, M.A. Perez, J.-A. Palma, L. Norcliffe-Kaufmann, H. Kaufmann

Department of Neurology, Dysautonomia Center, New York University School of Medicine, New York, NY, USA

Objective: To determine the frequency of seizures and epilepsy in patients with familial dysautonomia (FD, hereditary sensory autonomic neuropathy III).

Background: Seizure-like episodes are not uncommon in patients with FD but whether they are caused by epilepsy or by hypoxia and hypotension can be difficult to determine. Our aim was to categorize the EEG findings and the frequency of epileptiform activity in a large sample of patients with genetically confirmed FD.

Methods: Retrospective chart review of all cases with FD documented in the New York University FD Patient Registry, which includes virtually every patient diagnosed with FD from 1951 to 2018. We used the search terms "EEG", "epilepsy", and "seizure."

Results: Among 676 subjects in the NYU FD Patient Registry, we identified 426 adult and pediatric patients with diagnostic tests (EEG, polysomnography) for suspected seizures. Of these, almost one-third (32\%, 136 patients) had epileptiform EEG patterns; one-third (34\%, 143) had normal EEGs and the remaining one-third had non-epileptiform abnormalities including slowing, either focal $(4 \%, 15)$ or generalized $(14 \%, 60)$. In $9 \%$ (36 patients) the results stated "abnormal" without additional information and $1 \%$ (6) had beta activity possibly related to benzodiazepines. Of the 136 patients with epileptiform findings, almost half $(47.8 \%, 65)$ had focal findings and $18.4 \%$ (25) had seizure confirmed on their EEG. Over one-third of patients with paroxysmal events $(36.4 \%, 155$ of 426) were taking anti-epileptic drugs (AEDs) for presumed epilepsy. Almost $60 \%$ of those taking AEDs (58.7\%, 91 patients) had epileptiform findings on their EEG, and $12.3 \%$ (19) had seizures confirmed on their EEG. Conclusions: Approximately 1 in 3 patients with FD and paroxysmal episodes of altered consciousness have epileptiform activity on EEG. Hence, epilepsy is not uncommon in FD and should be considered in the differential diagnosis of some syncopal episodes.

Funding: Familial Dysautonomia Foundation. 


\section{Poster \#14}

Does renal denervation restore GABAergic tone in the paraventricular nucleus and thus attenuate the enhanced activation of the PVN in heart failure?

\section{K.P. Patel ${ }^{1}$, K. Katsurada ${ }^{1}$, X. Liu ${ }^{2}$, H. Zheng ${ }^{2}$}

${ }^{1}$ Department of Cellular and Integrative Physiology, University of Nebraska Medical Center, Omaha, NE, USA; ${ }^{2}$ Division of Basic

Biomedical Sciences, Sanford School of Medicine of the University of South Dakota, Vermillion, SD, USA

Previously we have shown that activated paraventricular nucleus (PVN) contributes to the sympatho-excitation in chronic heart failure (HF). Afferent renal nerves project to neurons in several brain areas, including the PVN, that are involved in the regulation of the cardiovascular system. The purpose of the present study was to determine if the activation of the PVN was mediated by afferent renal nerves. Chronic HF was induced by left coronary ligation surgery for 3-4 weeks and rats were surgically renal denervated (RDN) for 2 weeks. RDN resulted in reduced levels of urinary norepinephrine excretion in rats with HF compared with sham-operated control rats. RDN resulted in reduced levels of FosB staining in the PVN in rats with HF. RDN also resulted in restoration of nNOS levels (NOS histochemistry and nNOS immunostaining) in the PVN of rats with HF. We observed that microinjections of Bicuculline (GABA receptor blocker) into the PVN induced a significantly smaller increase in lumbar sympathetic nerve activity in anaesthetized rats with $\mathrm{HF}$ compared with sham rats. In contrast, RDN restored the Bicuculline responses in rats with HF. These results suggest that intact renal nerves contribute to the reduction of GABAergic tone within the PVN resulting in activation of the neurons in the PVN of rats with HF. RDN restores GABAergic tone within the PVN and thus attenuates the enhanced neuronal activation as well as the sympatho-excitation commonly observed in HF.

Funding: Supported by NIH grant HL62222 \& DK114663.

\section{Poster \#15}

Role of arterial baroreflex in respiratory sinus arrhythmia: a study using oscillatory lower body negative pressure in human subjects

A. Sood, S. Srivastav, D.S. Chandran, A.K. Jaryal, K.K. Deepak Department of Physiology, All India Institute of Medical Sciences, Ansari Nagar, New Delhi, India

Background: We investigated the role of arterial baroreflex in respiratory sinus arrhythmia (RSA) using oscillatory lower body negative pressure (oLBNP) to modulate blood pressure variability (BPV) during slow deep breathing at $0.1 \mathrm{~Hz}$, and measured its influence on heart rate variability (HRV) in the same frequency band.

Methods: HRV and BPV in 11 healthy human subjects (18-35 years) were measured under controlled conditions in three protocols during slow deep breathing $(0.1 \mathrm{~Hz} ; 3$ minutes/protocol): without LBNP application (control), with 'in-phase' oLBNP (LBNP applied during inspiration; intended to increase BPV), with 'out-of-phase' oLBNP (LBNP applied during expiration; intended to decrease BPV).

Results: Frequency domain analysis showed increase in low frequency (LF) power of BPV in $9(81.8 \%)$ subjects during in-phase oLBNP; and decrease in remaining $2(18.2 \%)(\mathrm{p}=0.05)$. Correlation analysis to compare delta changes in LF power of BPV and HRV from 22 recordings (11 each from in-phase and out-of-phase protocols) showed decrease in LF power of BPV in 7 (31.8\%) recordings; of these, $6(85.7 \%)$ recordings showed decrease and $1(14.3 \%)$ showed increase in LF power of HRV. Increase in LF power of BPV was seen in $15(68.2 \%)$ recordings; of these, $7(46.6 \%)$ showed increase and $8(53.4 \%)$ showed decrease in LF power of HRV. Overall, correlation analysis of these 22 recordings showed statistically significant correlation between LF power of BPV and HRV $(\mathrm{p}=0.0079$; spearman coefficient $(\mathrm{r})=0.56)$.

Conclusion: Oscillatory LBNP was only moderately successful in changing BPV as expected. Correlation analysis showed that BPV and HRV show parallel changes; lending support to the idea that arterial baroreflex contributes to genesis of RSA. The finding has clinical implication while interpreting an abnormal heart rate response to deep breathing test, as this could also be caused due to derangement in afferent baroreflex function.

Funding: This study was funded by Short Term Studentship program of the Indian Council of Medical Research, Govt. of India No. 2017-03318.

\section{Poster \#16}

The effect of dietary sodium intake on central blood pressure variability in young adults

K.U. Migdal ${ }^{1}$, J.C. Watso ${ }^{1}$, M.C. Babcock ${ }^{1}$, A.T. Robinson ${ }^{1}$, M.M. Wenner $^{1}$, S.D. Stocker ${ }^{2}$, W.B. Farquhar ${ }^{1}$

${ }^{1}$ Department of Kinesiology \& Applied Physiology, University of Delaware, Newark, DE, USA; ${ }^{2}$ Department of Medicine, University of Pittsburgh, Pittsburgh, PA, USA

Purpose: Central blood pressures (BP) are more closely correlated with cardiovascular (CV) risk than brachial BPs. In addition, high blood pressure variability (BPV) is correlated with CV risk. Rodent studies demonstrate that high dietary sodium increases BPV, yet the effects of dietary sodium on central BPV in humans is not known. Therefore, we tested the hypothesis that increased dietary sodium increases central BPV in young adults.

Methods: Seventeen adults (10M/7F; age: $26 \pm 5$ yrs; BMI: $25 \pm 4 \mathrm{~kg} /$ $\mathrm{m}^{2}$, Mean $\pm \mathrm{SD}$ ) participated in a controlled feeding study that consisted of ten days of low- (LS: $1.0 \mathrm{~g}$ sodium/day), medium- (MS: $2.3 \mathrm{~g}$ sodium/day), or high- (HS: $7.0 \mathrm{~g}$ sodium/day) sodium diets, in random order separated by at least a month. Central BPV was derived from 24-hour ambulatory BP monitoring (ABPM) using a validated generalized transfer function. Central BPV was calculated as average real variability (ARV; the average of the absolute differences between consecutive BP measurements). Data were analyzed using one-way repeated measures ANOVAs.

Results: Twenty-four-hour urinary sodium excretion (LS: $75 \pm 63$, MS: $99 \pm 66$, HS: $269 \pm 120 \mathrm{mmol} / 24$ hours, $\mathrm{P}<0.0001)$ and serum sodium (LS: $140.8 \pm 2.5$, MS: $141 \pm 2.5$, HS: $141.1 \pm 2.6 \mathrm{mmol} / \mathrm{l}$, $\mathrm{P}=0.01$ ) increased with increasing levels of dietary sodium. $24 \mathrm{~h}$ systolic central $\mathrm{BP}(\mathrm{P}=0.64)$ and brachial systolic $\mathrm{BP}(\mathrm{P}=0.37)$ was not different following the diets. Overall $24 \mathrm{~h}$ ABMP central systolic ARV was not different $(\mathrm{P}=0.06)$. However, in male participants, $24 \mathrm{~h}$ ABPM central systolic ARV increased with increasing levels of dietary sodium (LS: $9.3 \pm 1.9$, MS: $9.5 \pm 1.8$, HS: $11.0 \pm 2.6 \mathrm{mmHg}$, $\mathrm{P}=0.04)$. In female participants, $24 \mathrm{~h}$ ABPM systolic central ARV was not different (LS: $8.7 \pm 1.0$, MS: $8.6 \pm 1.4$, HS: $9.0 \pm 2.0 \mathrm{mmHg}$, $\mathrm{P}=0.80$ ).

Conclusion: These preliminary data suggest that 10 days of high dietary sodium intake may alter systolic central BPV in young male adults.

Funding: Supported by NIH Grant 1R01HL128388. 


\section{Poster \#17}

Relationships between cardiovascular disease, autonomic function, neuropathic pain, and depression following spinal cord injury

M.C. Dorton, S. de Groot, M. Post, J.J. Cragg, J.K. Kramer, V.E. Claydon

Biomedical Physiology and Kinesiology, Simon Fraser University, Burnaby, BC, Canada

Introduction: Cardiovascular disease (CVD) is the leading cause of morbidity and mortality in individuals with spinal cord injury (SCI). Neuropathic pain (NP) is common after SCI and may be associated with depression and a higher incidence of CVD following injury. Impaired autonomic function is a predictor of CVD risk and so may also be linked to the presence of NP. We aimed to examine the relationships between NP, CVD, autonomic function, and depression. Methods: We performed a multicentre cross-sectional study of individuals with chronic traumatic SCI $(n=257,61$ females, aged $47 \pm 9$ years, duration of injury [DOI] $23 \pm 9$ years). CVD risk was calculated using the Framingham risk score (FRS; $\mathrm{n}=227$ ). NP and depression were determined using DN4 $(n=133)$ and MHI-5 questionnaires $(n=240)$. Peak heart rate during maximal exercise (HRpeak; $n=156)$ was used as a measure of autonomic function.

Results: Most lesions were cervical $(45 \%)$ or thoracic $(50 \%) ; 71 \%$ were motor/sensory complete and $78 \%$ had lesions that could affect cardiovascular autonomic control. There was a high risk of CVD in $38 \%$ of participants, with $63 \%$ experiencing NP, $10 \%$ meeting criteria for depression, and $24 \%$ having impaired autonomic function (chronotropic incompetence [CI; HRpeak $\leq 125 \mathrm{bpm}]$ ). Akaike information criteria identified the following as important predictors for NP: higher FRS (importance 0.94, estimate 0.005), younger age at injury (importance 0.89 , estimate -0.04 ), MHI-5 score (importance 0.86 , estimate 0.003 ), and shorter DOI (importance 0.76, estimate $0.03)$.

Conclusions: This study highlights the increased CVD risk and high prevalence of NP following SCI. CVD risk and depression are important modifiable risk factors for NP. Sustaining an injury at a younger age, having a recent injury or high CVD risk increases the likelihood of NP. Impaired autonomic function seems to play a role in NP. Further research is needed to parse the relationships between NP, CVD, autonomic function, and depression following SCI.

Funding: Heart and Stroke R669045.

\section{Poster \#18}

\section{Slow breathing training reduces blood pressure even in healthy} normotensive subjects

\section{E.C. Smith, S. Paranjape, K. Nelson, A. Diedrich, G. Birdee, A. Gamboa Vanderbilt University Medical Center, Nashville, TN, USA}

Introduction: Slow breathing training, an integral part of body mind relaxation practices, might modulate autonomic tone. It is unknown if extended exhalation technique (Exhalation $>$ Inhalation, E $>\mathrm{I}$ ) will have different effects than common slow breathing $(E=I)$. We hypothesized that $\mathrm{E}>\mathrm{I}$ will have a greater impact on autonomic modulation and blood pressure.

Methods: We studied 86 healthy participants ( $41 \pm 4$ years, $76 \%$ female) randomly assigned to $\mathrm{E}=\mathrm{I}(\mathrm{n}=41)$ or $\mathrm{E}>\mathrm{I}(\mathrm{n}=45)$ daily exercise guided by Yoga teachers over 12 weeks. We measured blood pressure, autonomic tone by spectral analysis of heart rate and blood pressure variability and cardio-vagal baroreflex sensitivity by spontaneous sequence technique (BRS) at baseline and at 12 weeks. To adjust for the effect of repeated visits, we included two control groups without breathing interventions: normotensives (NTN) $(n=14$ SBP $114 \pm 5 \mathrm{mmHg})$ and hypertensives $(\mathrm{HTN})(\mathrm{n}=15 \mathrm{SBP} 141 \pm 3 \mathrm{mmHg})$, that were studied on two separate visits at least one month apart.

Results: SBP significantly decreased at 12 weeks for both slow breathing groups [decrease of $-3.5 \pm 6.6 \mathrm{mmHg}$ for $\mathrm{E}>\mathrm{I}(\mathrm{p}<0.001)$ and $-3.5 \pm 5.5 \mathrm{mmHg}$ for $\mathrm{E}=\mathrm{I}(\mathrm{p}<0.001)]$. There were no significant SBP changes for controls [increase of $3.5 \pm 8.4(\mathrm{p}=0.13)$ for HTN and decrease of $-1.6 \pm 8.1(\mathrm{p}=0.48)$ for NTN]. When participants were stratified by initial SBP in two groups: Higher SBP (HBP) $(\mathrm{SBP}=123 \pm 2 \mathrm{mmHg}, \mathrm{n}=24)$ and lower $\mathrm{SBP}$ (LBP) $(\mathrm{SBP}=96 \pm 1$ $\mathrm{mmHg}, \mathrm{n}=24)$. The SBP drop was significantly greater among HBP subjects: SBP decreased by $-6.3 \pm 7.3 \mathrm{mmHg}$ (p1 and $-0.24 \pm 0.8 \mathrm{~ms} /$ $\mathrm{mmHg}$ for $\mathrm{E}=\mathrm{I}, \mathrm{p}($ time $)=0.013$, $\mathrm{p}($ timeXgroup $)=0.019$.

Conclusion: In conclusion, 12 weeks of slow breathing training modulates autonomic tone and reduces blood pressure, especially among subjects with higher initial blood pressure.

\section{Poster \#19}

\section{Granulomatosis with polyangiitis presenting as autonomic failure}

B. Blech, M. Grill, B. Goodman

Department of Neurology, Mayo Clinic, Scottsdale, AZ, USA

Background: Granulomatosis with polyangiitis (GPA) is an autoimmune-mediated necrotizing granulomatous vasculitis affecting small to medium size vessels, and most commonly affects the respiratory tract and kidneys. Nervous system involvement is less common; objective findings of autonomic dysfunction in the setting of vasculitis are extremely rare. We report a case of GPA that presented as autonomic failure with resolution after immunotherapy.

Case Presentation: A 62-year-old woman developed autonomic symptoms including postural lightheadedness, syncope, dry mouth and heat intolerance 5 months prior to presentation. Subsequent symptoms included reflux, dysphagia, dysarthria and right facial numbness and weakness. Examination was notable for orthostatic hypotension, flaccid dysarthria, right orbicularis oris weakness, decreased sensation to pinprick and light touch in the V2-V3 distribution of the right trigeminal nerve, bilateral sensorineural hearing loss, diminished bilateral hypoglossal strength, and absent gag reflex. Autoimmune testing was significant for p-ANCA positivity and elevated MPO of 3.3 (negative $<0.4$ ). Autonomic reflex screen (ARS) showed widespread postganglionic sympathetic sudomotor, cardiovagal, and cardiovascular adrenergic impairment with severe orthostatic hypotension, consistent with an autonomic neuropathy and autonomic failure. MRI of the brain revealed diffuse posterior fossa pachymeningeal enhancement and thickening of the tentorium. EMG/ NCS showed evidence of multiple cranial neuropathies involving V, VII, and XII. Suboccipital dural biopsy revealed necrotizing granulomatous vasculitis consistent with GPA. Treatment consisted of intravenous methylprednisolone followed by rituximab infusions and maintenance prednisone. MRI two months later showed significant improvement in the posterior fossa dural enhancement, and repeat ARS showed complete resolution of dysautonomia, corresponding to clinical symptomatic improvement.

Conclusion: Our case illustrates a rare and unique presentation of GPA with autonomic failure followed by multiple cranial neuropathies. Mechanism for the autonomic failure likely relates to an autonomic ganglionopathy, such as ganglionitis or vasculitic process, although little on this topic has been reported in the literature, especially in GPA. 


\section{Poster \#20}

\section{Quality improvement and practice-based research in diabetic} autonomic neuropathy: preliminary observations

A. Barboi, O. Kincaid, S. Pocica, S. Tideman, L. Garduno, V. Patel, L. Hillman, D. Macapinlac, R. Frigerio, K.C. Simon, D. Maraganore Department of Neurology, NorthShore University HealthSystem, Evanston, IL, USA

Introduction: We used our electronic medical record (EMR) based documentation support (SCDS) toolkit to describe a diabetic autonomic neuropathy (DAN) cohort.

Methods: We compared patient-reported outcomes: the Center for Epidemiological Studies-Depression (CES-D) scale, Fatigue Severity Scale (FSS), Survey of Autonomic Symptoms (SAS), Insomnia Severity Index (ISI) with physician entered autonomic and sensory symptom profiles, clinical examination findings and autonomic reflex screen (ARS) results in 140 patients with DAN.

Results: The DAN patients were selected from 327 patients currently enrolled in our neuropathy project. There were 53 women and 87 men with a median age of $66.56 .4 \%$ were Caucasian, $6.4 \%$ were African American, $6.4 \%$ Hispanic/Latino, and $2.9 \%$ were Asian. At least one autonomic symptom was present in $85 \%$ and at least one somatic neuropathic symptom in $91.4 \%$. For patient-reported outcomes: $42.9 \%$ had depression, $57.1 \%$ fatigue, $50.7 \%$ insomnia. Autonomic symptoms strongly correlated with fatigue. There was a moderate correlation between the SAS score and autonomic symptoms entered by the physician $(\mathrm{P}<0.002)$ and with abnormalities found on ARS $(\mathrm{P}<0.016)$. Autonomic symptoms, in general, were strongly correlated with fatigue $(\mathrm{p}<0.006)$. The higher the SAS score, the more likely it was to have bowel, bladder, and sexual dysfunction whereas lower SAS scores correlated with orthostatic hypotension and sudomotor dysfunction. Cardiovagal dysfunction predicted the coexistence of sympathetic cardiac more often than vasomotor dysfunction. Quantitative loss of sweat was equally predictive of cardiac vagal and sympathetic dysfunction. Pain was not predicted by the SAS but had a significant correlation with the presence of sensorimotor symptoms. Higher risk of falls was predicted more by increasing the severity of sensory loss in the leg than by the presence of orthostatic hypotension. Conclusion: We believe our SCDS toolkit will allow robust longitudinal evaluation of DAN and offer insight into treatment strategies. Funding: Targeted study funding is provided by the Agency for Healthcare Research and Quality grant 1R01HS024057-01 "Quality Improvement and Practice-Based Research in Neurology Using the EMR".

\section{Poster \#21}

\section{Clinical features of autoimmune gastrointestinal dysmotility in Japan}

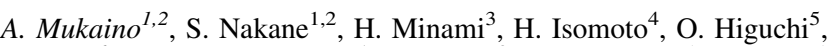
E. Ihara ${ }^{6}$, A. Ido ${ }^{7}$, H. Matsuo ${ }^{5}$, K. Nakao ${ }^{3}$, T. Yamashita ${ }^{1}$, Y. Ando ${ }^{1}$ ${ }^{1}$ Department of Neurology, Kumamoto University, Kumamoto, Japan; ${ }^{2}$ Department of Molecular Neurology and Therapeutics, Kumamoto University Hospital, Kumamoto, Japan ${ }^{3}$ Department of Gastroenterology and Hepatology, Nagasaki University, Nagasaki, Japan; ${ }^{4}$ Division of Medicine and Clinical Science, Department of Multidisciplinary Internal Medicine, Tottori University, Tottori, Japan; ${ }^{5}$ Department of Clinical Research and Neurology, Nagasaki Kawatana Medical Center, Kawatana, Japan; ${ }^{6}$ Department of Medicine and Bioregulatory Science, Kyushu University, Fukuoka,
Japan; ${ }^{7}$ Digestive and Lifestyle Diseases, Department of Human and Environmental Sciences, Kagoshima University, Kagoshima, Japan

Background: Autoimmune gastrointestinal dysmotility (AGID) is the disease entity caused by autoantibodies including achalasia, gastroparesis and chronic intestinal pseudo-obstruction. AGID is also reported to be a limited form of autoimmune autonomic ganglionopathy (AAG) and anti-ganglionic acetylcholine receptor (gAChR) antibody is one of the pathogenic antibodies.

Objective: To elucidate the clinical features of AGID in Japan.

Method: 1) Retrospective study: We investigated the frequency, clinical features, and treatment responsiveness of AGID including esophageal dysfunction, gastroparesis and paralytic ileus in 123 cases of anti-gAChR antibody positive AAG. 2) Prospective study: We investigated the frequency of anti-gAChR antibody, clinical features, and COMPASS 31, which is severity score of autonomic symptoms in 28 cases of achalasia and 14 cases of CIPO. We measured anti- $\alpha 3$ and $\beta 4 \mathrm{gAChR}$ antibodies by luciferase immunoprecipitation systems test. Results: 1) We confirmed that 6 cases of esophageal dysfunction including achalasia, 2 cases of gastroparesis, and 2 cases of paralytic ileus in 123 anti-gAChR antibody positive AAG, and experienced the immunotherapy-responsive case of gastrointestinal motility. 2) Six cases $(21.4 \%)$ of achalasia and seven cases $(50 \%)$ of CIPO were seropositive. Seropositive CIPO was frequently observed bladder dysfunction in COPASS31.

Conclusion: Severe gastrointestinal motility disorder is present in seropositive AAG in Japan, and in some cases, efficacy by immunotherapy has been shown. Seropositive cases were also found in the achalasia and CIPO cases. For these neurogastroenterology and GI motility disorders, neurologists and gastroenterologists should cooperate with each other to decide the treatment strategy.

Funding: This study was supported by the Ministry of Health, Labor, and Welfare, Japan, and the Ministry of Education, Culture, Sports, Science, and Technology of Japan (JSPS KAKENHI Grant Number 25461305).

\section{Poster \#22}

Quantification of vasodilation and sweating responses during heat stress in a person with and without spinal cord injury: a case report

M.T. Trbovich, R.C. Camacho

Department of Rehabilitation Medicine, University of Texas Health Science Center at San Antonio, San Antonio, TX, USA

Introduction: Vasodilation (VD) and sweating response (SR) are the main methods of heat dissipation employed under high thermal strain. After spinal cord injury (SCI), it is well accepted that thermoregulation during heat stress is impaired, however, quantification of the change in VD and SR and its relation to neurological level of injury has not been widely investigated. In able-bodied (AB) persons, VD and SR appear to involve the same nerves, while data in persons with SCI suggest reports discordance between VD and SR. The goal of this case report was to quantify and compare VD and SR in $\mathrm{AB}$ and a person with tetraplegia (TP); and to determine if VD and SR are correlated with the sensorimotor level of injury.

Case Presentation: A 30-year-old AB and 23-year-old TP (C3 AIS B) underwent passive heat stress. VD was quantified over $\mathrm{C} 2, \mathrm{C} 4$ and $\mathrm{C} 5$ dermatomes using laser doppler flowmetry and SR over the C2-T5 dermatomes via the starch iodine test. The TP demonstrated remaining but very diminished VD responses above and below the level of injury, compared to AB. TP did not sweat while AB sweated diffusely. 
Discussion: There was a large difference in VD and SR under passive heat stress between participants. Intact VD and absence of SR in TP suggests an anatomic dissociation in the neurological control pathways, while impaired VD and SR above and below level of injury in the TP supports that sensorimotor level of injury cannot precisely predict remaining thermoregulatory function.

Funding: Veterans Affairs, Rehabilitation Research and Development, Career Development Award \#RX001805.

\section{Poster \#23}

Is there an association between resting heart rate and cardiovagal function early-to-late phases of recovery after resistance training?

G.L. Garcia, C.J.G. Cruz, E.M.K.V.K. Soares, M.T. Morlin, L.F. Junqueira Jr., L.G.G. Porto, G.E. Molina

Exercise Physiology Laboratory, University of Brasília, UnB, Brasília, Brazil

Introduction: The high values of resting heart rate (RHR) and low degree of vagal cardiac autonomic modulation (CAM) during the recovery period after exercise are related to higher morbidity and mortality in apparently healthy men. However, the association between RHR and CAM during early-to-late phase transition after resistance training (RT) is not yet fully understood.

Purpose: To correlate RHR and CAM during early-to-late phase transition after resistance training in healthy men.

Methods: We evaluated 14 clinically normal men, aged $24.1 \pm 5.6$ years and showing BMI $=24.9 \pm 1.9 \mathrm{~kg} / \mathrm{m}^{2}$. A Polar RS800® was used to record HR at rest and heart rate (HR) and the RR-interval series during 5 (early recovery), 10 (mid recovery) and 15 (late recovery) minutes during recovery period after RT. RHR was defined as the mean heart rate recorded for 5 minutes at rest in supine position after 10 minutes of resting. The RT session was composed of 3 sets of 8-12 RM with 1 min of rest interval between sets, in the leg press exercise. The heart rate variability was analyzed by Kubios software and vagal CAM was assessed by r-MSSD, p-NN50 and SD1, all vagal markers, during passive supine recovery after RT. Due to non-compliance with normality assumptions (Shapiro-Wilk test) the Spearman correlation test was used to correlate RHR with CAM during early-to-late phase transition after RT, at the 5\% level of significance.

Results: We observed a positive correlation between RHR with HR in the five ( $r s=0.75, p=0.001)$, ten $(r s=0.66, p<0.01)$ and fifteen $(r s=0.77$, $\mathrm{p}<0.001$ ) minutes of recovery after RT. And, we observed a negative correlation between RHR with all vagal markers in the five ( $r s=-0.70$, $\mathrm{p}<0.01)$, ten $(\mathrm{rs}=-0.66, \mathrm{p}<0.01)$ and fifteen $(\mathrm{rs}=-0.60-0.66, \mathrm{p} \leq 0.01)$ minutes of recovery after RT.

Conclusion: We observed the significant positive correlation between the RHR and HR during early-to-late phase transition after RT and significance negative correlation between RHR with CAM during early-to-late phase transition after RT in healthy men. This is the first paper to show this phenomenon using RT as cardiovascular stressor. Therefore, these results support the hypothesis that pre-exercise RHR in supine position could predict the degree of cardiovagal reactivation and, consequently, the speed of cardiovagal activity return to baseline levels after RT, then, our results demonstrate that the lower is the RHR the lower is the HR and higher is the vagal modulation after RT. Funding: Fundação de apoio a pesquisa do Distrito Federal- FAPDF.

\section{Poster \#24}

\section{Evolution of pulmonary hemodynamics during severe sustained} hypoxia

F. Hoffmann ${ }^{1}$, U. Limper ${ }^{2}$, V. Zaha ${ }^{3}$, H. Reuter ${ }^{4}$, L. Zange ${ }^{5}$, J. Schulz-Menger $^{6}$, M. Hein ${ }^{7}$, H. Sadek ${ }^{8}$, S. Baldus ${ }^{9}$, B. Levine ${ }^{10}$, J. Jordan $^{2}$,

J. Tank ${ }^{2}$

${ }^{1}$ Department of Cardiology, Internal Medicine III, University of Cologne, Germany; ${ }^{2}$ Institute of Aerospace Medicine, German Aerospace Center, Cologne, Germany; ${ }^{3}$ Department of Anesthesiology and Intensive Care Medicine, Cologne-Merheim Medical Center, University of Witten Herdecke, Germany; ${ }^{4}$ The University of Texas Southwestern Medical Center, Dallas, TX, USA;

${ }^{5}$ Working Group on Cardiovascular Magnetic Resonance, Experimental and Clinical Research Center, Berlin, Germany; ${ }^{6}$ Department of Cardiology and Nephrology, HELIOS Hospital Berlin-Buch, Berlin, Germany; ${ }^{7}$ Department of Anesthesiology, Medical Faculty, RWTH Aachen University, Germany; ${ }^{8}$ Department of Internal Medicine, University of Texas Southwestern Medical Center, Dallas, Texas, USA; ${ }^{9}$ Center for Regenerative Science and Medicine, University of Texas Southwestern Medical Center, Dallas, TX, USA; ${ }^{10}$ Division of Cardiology, Department of Internal Medicine, University of Texas Southwestern Medical Center, Dallas, TX, USA

Background: A human feasibility study testing influences of normobaric hypoxia corresponding to $>7,000 \mathrm{~m}$ altitude on the heart allowed us to follow the evolution of pulmonary hypertension.

Methods: After pre-acclimatization, two healthy mountaineers stayed in a hypoxia chamber, where normobaric hypoxia was achieved by nitrogen dilution starting at $13.5 \%$ to a minimum of $8 \% \mathrm{O}_{2}$ over three weeks. Afterwards, $8.5 \%$ during daytime and $8.8 \% \mathrm{O}_{2}$ at night was kept for 14 days. Velocity encoded single-plane phase contrast MRI of the pulmonary artery (PA) and echocardiography were performed 1 month before, during and 1 and 3 months after hypoxia for assessment of right ventricular (RV) function, PA pressure, area and distensibility.

Results: Systolic PA pressure (PAPsys) was closely related to decreasing oxygen levels. In subject B PAPsys peaked at $67 \mathrm{mmHg}$, $48 \mathrm{mmHg}$ higher than baseline. PAPsys decreased steadily to 44 $\mathrm{mmHg}$ after two weeks while still at $8.55 \%$ oxygen. Peak values occurred with clinical symptoms of acute mountain sickness but without signs of cerebral or pulmonary edema. In subject A peak PAPsys was $44 \mathrm{mmHg}$ and remained stable. RV function was preserved in both subjects. In subject B PA-area showed dilation with a maximum relative change in respect to baseline of 50\% and an absolute change of $4.03 \mathrm{~cm}^{2}$. PA-distensibility decreased with hypoxia by a maximum of $57 \%$. After 30 hours of reconditioning to normoxia, all values went back to baseline levels in both subjects. Conclusion: In healthy humans, severe hypoxia induces pulmonary hypertension with preserved right ventricular function. Pulmonary hypertension may improve over time in some individuals. The findings attest to the physiological reserve of healthy humans allowing for adaptation to increased pulmonary pressure.

Funding: The study was funded by internal funds. FH was supported by the University Hospital of Cologne, 50WB1816. 


\section{Poster \#25}

Early-onset blood pressure and muscle sympathetic nerve activity responsiveness to cold pressor test in young, healthy adults

\section{H.A. Cunningham, J.R. Carter}

Michigan Technological University, Houghton, MI, USA

While the cold pressor test (CPT) elicits robust and consistent increases in blood pressure (BP), heart rate, and muscle sympathetic nerve activity (MSNA), the patterns of response can vary considerably. Specifically, we have noted that during CPT onset (i.e., initial 15-30 seconds), some subjects appear to have a brief depressor response that recovers quickly, while others exhibit a sudden rise of BP (i.e., pressor response). The purpose of the present study was to compare the MSNA responsiveness in subjects with early-onset depressor verses pressor responses during CPT. Forty-three young, healthy adults $(21 \pm 1 \mathrm{yrs} ; 20$ men and 23 women) participated in an experimental visit where continuous BP (finger plethysmography) and MSNA (microneurography) were measured during a 3-5 min baseline and 2-min CPT. The initial 15 seconds of CPT were used to separate subjects for analysis based on mean arterial pressure (MAP) responsiveness, which resulted in the following two groups: 1) early-onset pressor group $(\mathrm{n}=28 ; 18$ women; mean, $\Delta 5 \pm 1 \mathrm{mmHg}$; range $\Delta 0-$ $20.6 \mathrm{mmHg}$ ), 2) early-onset depressor group ( $\mathrm{n}=15 ; 5$ women; mean, $\Delta-5 \pm 3 \mathrm{mmHg}$; range $\Delta-10.7-0 \mathrm{mmHg}$ ). While there was a significant time $\times$ group interaction for systolic $(\mathrm{p}<0.001)$, diastolic $(\mathrm{p}=0.001)$, and mean $(\mathrm{p}=0.001) \mathrm{BP}$, all groups demonstrated similar MSNA reactivity patterns when expressed as burst frequency (time $\times$ group, $\mathrm{p}=0.508$ ), bursts per 100 heart beats (time $\times$ group, $\mathrm{p}=0.627$ ), and total MSNA (time $\times$ group, $\mathrm{p}=0.602$ ). Consistent with this finding, we found no correlation between $\triangle \mathrm{MAP}$ and $\triangle \mathrm{MSNA}$ during early-onset CPT $(p=0.408 ; r=-0.129)$. Heat rate was also similar between groups (time $\times$ group, $\mathrm{p}=0.722$ ). The primary findings (i.e., altered BP, but not MSNA, responsiveness) were similar when analyzed using a tertile analysis that resulted in smaller (i.e., $n=14-15$ per tertile) yet more dramatic pressor and depressor groups. In summary, the early-onset divergence of BP reactivity to CPT does not appear to be related to changes in MSNA.

Funding: Support for this study provided from the National Institutes of Health (HL098676, HL088689, AA024892) and the Portage Health Foundation.

\section{Poster \#26}

Cardiorespiratory response to the exercise pressor reflex in women through the menstrual cycle with or without oral contraceptive use

I. Ivry, E. Assadpour, S. Wasef, B. Adeyinka, H. Edgell School of Kinesiology, York University, Toronto, ON, Canada

Recently our group found that women do not increase ventilation during post-exercise circulatory occlusion (PECO; Joshi and Edgell, 2019). However, activation of the exercise pressor reflex (combined metaboreflex and mechanoreflex) increases ventilation. Therefore, we investigated both reflexes in women while investigating the menstrual cycle and oral contraceptive (OC) use. Twelve women not taking OC (NOC; age: $22.5 \pm 3.5$, BMI: $23.8 \pm 2.9 \mathrm{~kg} / \mathrm{m}^{2}$ ) and ten women taking OC (age: $22.9 \pm 5.0$, BMI: $24.8 \pm 3.6 \mathrm{~kg} / \mathrm{m}^{2}$ ) were recruited. Women were asked to participate in the low hormone (LH; Day 2-5 for NOC, placebo pill for OC) and high hormone (HH; Day 18-24 for NOC, last week of active pill for OC) phases of the menstrual cycle. Two trials were randomized 1) 2 minutes of $40 \%$ MVC handgrip followed by 3 minutes of PECO, and 2) 3 minutes of passive leg movement (PLM; 1 $\mathrm{Hz}$ ). Continuous blood pressure and ventilation were measured. Mean arterial blood pressure (MAP) was higher during PECO compared to baseline in all conditions with no differences between groups (NOCLH: $83 \pm 7$ to $99 \pm 14 \mathrm{mmHg}$; NOC-HH: $88 \pm 17$ to $105 \pm 18 \mathrm{mmHg}$; OC-LH: $87 \pm 6$ to $98 \pm 10 \mathrm{mmHg}$; OC-HH: $88 \pm 8$ to $96 \pm 8 \mathrm{mmHg}$; $\mathrm{P}>0.05$ ). MAP did not change throughout PLM in any group $(\mathrm{P}>0.05)$. Ventilation was higher in OC compared to NOC for both trials $(\mathrm{P}<0.001)$ and did not increase above baseline during PECO in any group (NOC-LH: $10 \pm 2$ to $11 \pm 3 \mathrm{~L} / \mathrm{min}$; NOC-HH: $11 \pm 3$ to $11 \pm 3$ L/min; OC-LH: $13 \pm 6$ to $15 \pm 4 \mathrm{~L} / \mathrm{min}$; OC-HH: $12 \pm 2$ to $13 \pm 2 \mathrm{~L} / \mathrm{min}$; $\mathrm{P}=0.35)$. Ventilation increased from baseline for only the first minute of PLM with no differences between groups (NOC-LH: $10 \pm 2$ to $12 \pm 2 \mathrm{~L} / \mathrm{min}$; NOC-HH: $10 \pm 2$ to $13 \pm 6 \mathrm{~L} / \mathrm{min}$; OC-LH: $10 \pm 2$ to $12 \pm 3 \mathrm{~L} / \mathrm{min}$; OC-HH: $12 \pm 3$ to $14 \pm 3 \mathrm{~L} / \mathrm{min}$; $\mathrm{P}<0.05$ ). Our results support previous findings of no ventilatory response to PECO women while finding that women do have a ventilatory response to PLM. We have also found that neither OC nor menstrual phase influence the metaboreflex or the mechanoreflex.

Funding: Funding was provided by the Natural Sciences and Engineering Council of Canada (05289).

\section{Poster \#27}

Effects of obstructive sleep apnea on baroreflex gain in patients with hypertrophic cardiomyopathy

S. Venkataraman, A. Rajendran, J. Bukartyk, N. Covassin, J.B. Geske, M. Mansukhani, A.C. Chahal, V.K. Somers

Department of Cardiovascular Medicine, Mayo Clinic, Rochester, MN, USA

Introduction: Patients with hypertrophic cardiomyopathy (HCM) are at high risk for both arrhythmias and sudden cardiac death (SCD), thus there is an important need of biomarkers to predict such risks in HCM. Baroreflex impairment is associated with increased arrhythmia and SCD risk. Patients with obstructive sleep apnea (OSA) have impaired baroreflex function and also have an elevated risk of arrhythmias and SCD. Screening studies in HCM patients suggest a high prevalence of OSA.

Hypotheses: HCM patients have a high prevalence of OSA. Untreated OSA in HCM results in impaired baroreflex gain and hence may increase the likelihood of adverse outcomes.

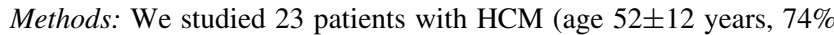
males) for the presence and absence of OSA with an in-lab overnight polysomnography. We measured baroreflex sensitivity (BRS) to determine whether HCM patients with comorbid OSA have an attenuated baroreflex gain compared to HCM patients without OSA. Baroreflex function was assessed during resting spontaneous breathing by evaluating RR responses to BP fluctuations using the sequence method.

Results: Sixty-five percent $(65.2 \%$; $\mathrm{n}=15)$ of HCM patients had previously undiagnosed OSA. There was no difference in mean systolic or diastolic BP in HCM without OSA vs. HCM with OSA (122 \pm 6 $\mathrm{mmHg}$ vs. $121 \pm 5 \mathrm{mmHg}, \mathrm{P}=0.88 ; 75 \pm 4 \mathrm{mmHg}$ vs. $75 \pm 3 \mathrm{mmHg}$, $\mathrm{P}=0.91$, respectively). Baroreflex gain was comparable in $\mathrm{HCM}$ patients with OSA vs. HCM without OSA $(19.9 \pm 17 \mathrm{~ms} / \mathrm{mmHg}$ vs. $20.3 \pm 5 \mathrm{~ms} / \mathrm{mmHg}, \mathrm{P}=0.9$ ). 
Conclusion: There was a high prevalence of OSA in HCM. This study suggests that a large proportion of patients without HCM have comorbid and previously undiagnosed OSA. However, BRS in HCM patients with OSA is very similar to BRS in HCM patients without OSA. Therefore, depressed baroreflex gain would not appear to be potentially implicated in any increased risk of arrhythmias and SCD in HCM patients with OSA.

Funding: NIH R01 HL134885-01.

\section{Poster \#28}

HIV-related deficits in volume of the central autonomic network and resting cardio-autonomic tone

R. McIntosh, J. Paparozzi, D. Qadir, D. Dukenik, G. French, J. Lobo Department of Psychology, University of Miami, College of Arts and Sciences, Miami, FL, USA

Introduction: Structural magnetic resonance imaging (MRI) studies increasingly provide support for a link between cortical thickness of brain regions in the central autonomic network (CAN) and heart rate variability (HRV) at rest. Chronic HIV infection is associated with an accelerated rate of cortical thinning as well as decrements in multiple indices of autonomic function. However, these factors have not been investigated concurrently. Moreover, sex and age mitigate the link between cortical thickness and HRV.

Methods: Structural MRI and resting HRV data from an ethnically diverse sample of 26 post-menopausal women living with HIV (age mean=55.92) and $53 \mathrm{HIV}$-negative controls (age mean=59.73) were concatenated from a cohort recruited locally and through a publically available neuroimaging repository. T1-weighted structural images were spatially pre-processed using the Diffeomorphic Anatomical Registration Exponentiated Lie algebra (DARTEL) program in SPM12. Gray matter volumes of interest from the CAN, i.e., amygdala, $\mathrm{R} / \mathrm{L}$ anterior insula, ventromedial prefrontal cortex (VMPFC) and dorsal anterior cingulate, were extracted for group comparison. Inter-beat intervals were collected over 5-minutes at rest using an MRI-compatible photoplethysmogram (PPG). Power frequency spectrum was quantified into high-frequency (HF, 0.15-0.4 Hz) and low-frequency (LF, $0.04-0.15 \mathrm{~Hz}$ ) domains. LF/HF ratio was regressed on individual total intracranial volume maps.

Results: Controlling for total intracranial volume and age, HIV+ women showed lower volume of the left anterior insula $F(1,75)=8.10$, $\mathrm{p}=0.006$. Gray matter regions of interest (ROI) were largely unrelated to LF, HF, and LF:HF (all p's >0.05). However, after controlling for age and HIV+ status, greater LF:HF was associated with greater volume of voxels distributed throughout the thalamus, midbrain, brainstem, and cerebellum ( $\mathrm{p}<0.001$, FWE-corrected).

Conclusion: Sympathoexcitatory and cardiac acceleratory circuits are prominent throughout the midbrain and brainstem. Greater volume of these subcortical ROIs correlated with higher sympathetic tone. Understanding the relationship between autonomic function and brain morphology may provide important insight into neural mechanisms underlying dysautonomia and neurodegenerative disease.

Funding: National Heart Lung \& Blood Institute, K01 HL139722.

\section{Poster \#29}

Specific cerebral multiple sclerosis lesions alter cardiovascular autonomic modulation

K. Winder, R.A. Linker, R. Wang, T. Engelhorn, A. Dörfler, K. Fröhlich, M.J. Hilz

Friedrich-Alexander University Erlangen-Nürnberg, Erlangen, Germany

Introduction: Autonomic dysfunction is common in multiple sclerosis (MS) and might be related to cerebral MS-lesion-sites. We applied voxel-based lesion-symptom mapping (VLSM) to determine associations between cardiovascular autonomic dysfunction and cerebral MS-related lesion-sites.

Methods: We studied 74 MS-patients (mean age $37.0 \pm 10.5$ years) and recorded electrocardiographic RR-intervals and systolic and diastolic blood pressure. We assessed low (0.04-0.15 Hz) and high $(0.15-0.5 \mathrm{~Hz})$ frequency RR-interval- and blood pressure-oscillations and determined parasympathetically-mediated RR-interval-high-frequency modulation, mainly sympathetically-mediated RR-intervallow-frequency modulation, sympathetically-mediated blood pressurelow-frequency modulation, and the ratios of sympathetic and parasympathetic RR-interval-modulation reflecting sympatheticparasympathetic balance. We analyzed cerebral MS-lesions on magnetic resonance imaging scans and performed a VLSM-analysis correlating parameters of autonomic dysfunction with cerebral MSlesion sites. Autonomic parameters were correlated with potential confounding factors of autonomic modulation age, disease-duration and -severity, and total cerebral lesion-volume (Spearman-rank-correlation; significance: $\mathrm{p}<0.05)$.

Results: The VLSM-analysis showed associations between increased RR-interval low-frequency-high frequency ratios and lesions in the left insular, hippocampal, and right frontal inferior-opercular region, and a smaller lesion-cluster in the right middle cerebellar peduncle. Increased blood pressure-low-frequency powers were associated with lesions primarily in the right posterior parietal white-matter and again left insular region. RR-interval low-frequency/high-frequency ratios and systolic blood pressure-low-frequency powers did not correlate with potential confounding factors.

Conclusion: Our data indicate associations between a shift of cardiovascular sympathetic-parasympathetic balance towards increased sympathetic modulation and left insular and hippocampal lesions, areas that normally modulate parasympathetic function. The VLSManalysis differentiated between right inferior-fronto-opercular lesions disinhibiting cardiac sympathetic activation and right posterior-parietal lesions increasing sympathetic blood pressure-modulation.

\section{Poster \#30}

Artificial intelligence algorithms and natural language processing for the recognition of syncope patients in emergency department medical records

F. Dipaola ${ }^{1}$, M. Gatti ${ }^{2}$, D. Shiffer ${ }^{1}$, V. Pacetti ${ }^{3}$, A.G. Bottaccioli ${ }^{4}$, M. Minonzio ${ }^{1}$, R. Menè ${ }^{1}$, M. Solbiati ${ }^{5}$, G. Costantino ${ }^{5}$, M. Anastasio ${ }^{6}$, E. Sini ${ }^{6}$, F. Barbic ${ }^{1}$, E. Brunetta ${ }^{1}$, R. Furlan ${ }^{1}$

${ }^{1}$ Internal Medicine, Humanitas Clinical and Research Center IRCCS, Biomedical Sciences Department, Humanitas University, Rozzano, Milan, Italy; ${ }^{2}$ IBM Italy, Segrate, Milan, Italy; ${ }^{3}$ Centro Trombosi e Malattie Emorragiche, Humanitas Clinical and Research Center IRCCS, Rozzano, Milan, Italy; ${ }^{4}$ Faculty of Psychology, "Vita-Salute San Raffaele" University, Milan, Italy; ${ }^{5}$ Pronto Soccorso e Medicina D’Urgenza, Fondazione IRCCS Ca' Granda Ospedale Maggiore 
Policlinico, Università degli Studi di Milano, Milan, Italy; ${ }^{6}$ ICT Department, Humanitas Clinical and Research Center IRCCS, Rozzano, Milan, Italy

Background: Since syncope-related adverse events are rare, it is necessary to enroll large cohorts of patients to optimize risk stratification and improve overall quality of patient management.

Objective: To develop natural language processing algorithms for identifying syncope episodes in emergency department (ED) medical records.

Methods: De-identified electronic medical records of all consecutive patients, $\geq 18$ years, evaluated at Humanitas Research Hospital ED between December 1st, 2013 to March 31st, 2014 and between December 1st, 2015 to March 31st, 2016, were analyzed. Manual annotation was performed to identify syncope. Emergency physicians' judgement was considered the "reference standard" for syncope diagnosis. All records were combined in a single dataset and were used for the machine learning algorithms, regardless of the annotation results. Classification of records was performed by developing multiple feature extractor algorithms, both manual and automatic, and testing their performance in combination with multiple classifiers. The main outcomes were accuracy, sensitivity, specificity, positive predictive value, negative predictive value and F3 score. Algorithms performances were compared using a Welch Two Sample t-test.

Results: We manually analyzed 15,098 records from the 2013 dataset and 15,222 from the 2015 dataset. Syncope was present in 571 (1.9\%) records. The algorithm entailing both the extractor Normalized Gini Index and the classifier Support Vector Machines conveyed the best F3 value of $84.0 \%$, corresponding to $92.2 \%$ sensitivity and $47.4 \%$ positive predictive value. This model performed significantly better than the combination with Humanitas manual extractor ( $\mathrm{F} 3$ value, $84.0 \%$ vs. $78.8 \%$; difference, $5.2 \%$; $95 \%$ CI, $-8.3-2.1 ; \mathrm{p}=0.005$ ).

Conclusions: This algorithm allowed the rapid selection of large populations of syncope patients using medical records generated during routine clinical activities. If our results were to be confirmed at external validation, the proposed tool could provide a huge amount of data suitable for prognostic stratification, thus resulting in an improvement in syncope management and outcome.

\section{Poster \#31}

Forearm vascular resistance responses to the Valsalva maneuver

\section{B.C.D. Hockin, E.Z. Tang, M.G. Lloyd, V.E. Claydon}

Department of Biomedical Physiology and Kinesiology, Simon Fraser University, Burnaby, BC, Canada; International Collaboration on Repair Discoveries (ICORD), University of British Columbia, Vancouver, BC, Canada

Introduction: In response to orthostatic reductions in blood pressure (BP), baroreflex-mediated vasoconstriction is crucial for effective autonomic BP control and prevention of syncope. Characterization of vasoconstrictor capacity is critical to understand the mechanisms underlying syncope. The Valsalva maneuver (VM) induces BP decreases that evoke baroreflex-mediated vasoconstriction. We hypothesized that individuals with smaller vascular responses during the VM would be more susceptible to syncope.

Methods: Healthy younger ( $\mathrm{N}=34 ; 16$ males; age $26.0 \pm 4.7$ years) and older ( $\mathrm{N}=18$; 9 males; age $62.6 \pm 9.8$ years) adults performed a supine $40 \mathrm{mmHg}, 20 \mathrm{~s} \mathrm{VM}$. A $60^{\circ}$ head-up-tilt test with combined, graded lower body negative pressure continued to presyncope was used to determine orthostatic tolerance (OT) in minutes (susceptibility to syncope). Non-invasive beat-to-beat BP and heart rate (Finometer Pro) were recorded continuously. Forearm vascular resistance (FVR) was calculated as mean arterial pressure divided by brachial blood flow velocity (Doppler ultrasound) relative to baseline.

Results: The mean OT was $26 \pm 1.4 \mathrm{~min}$. The VM produces a distinctive FVR pattern that peaks $(+127 \pm 12 \%)$ in phase $2 \mathrm{~B}(16.5 \pm 0.2$ $\mathrm{sec})$ as the baroreflex responds to low pressure perturbations. The maximum FVR response, expressed relative to the BP decrease in phase 2A (FVRmax/MAP2A-1), was correlated with age $(\mathrm{R}=-0.352$; $\mathrm{p}=0.01)$ and OT $(\mathrm{R}=-0.350 ; \mathrm{p}=0.01)$. There was a main effect of age $(\mathrm{p}<0.001)$ on maximum FVR, particularly in females, with older women $(+244 \pm 32 \%)$ mounting larger FVR responses than younger women $(+87 \pm 14 \% ; \mathrm{p}<0.001)$. Furthermore, there was an effect of sex in the older age group, with older women mounting larger FVR responses than older men $(+139.3 \pm 23.3 \% ; \mathrm{p}=0.015)$. In women, FVRmax/MAP2A-1 was significantly related to the maximum FVR attained during tilt $(\mathrm{R}=-0.528 ; \mathrm{p}=0.008)$.

Conclusion: This is the first characterization of FVR responses to the VM. The VM elicits large baroreflex-mediated increases in FVR in response to the BP dip in phase 2A. Small FVR responses to the VM may indicate susceptibility to syncope.

Funding: Heart and Stroke Foundation (HSFC G-18-0021).

\section{Poster \#32}

\section{Patient-reported "good" days during a prospective study of the treatment of neurogenic orthostatic hypotension with droxidopa}

\section{S. Kymes ${ }^{1}$, L.A. Hewitt ${ }^{2}$, C. François ${ }^{3}$}

${ }^{1}$ Health Economic and Outcomes Research, Lundbeck, Deerfield, IL, USA; ${ }^{2}$ US Medical, Lundbeck, Deerfield, IL, USA; ${ }^{3}$ Health Economic and Outcomes Research, Creativ-Ceutical, Paris, France

Objective: To evaluate the effect of droxidopa treatment on patientreported assessments of a "good" or "bad" day in patients with neurogenic orthostatic hypotension $(\mathrm{nOH})$.

Background: Droxidopa is approved in the United States to treat symptomatic $\mathrm{nOH}$ in adults. Common symptoms of $\mathrm{nOH}$ include lightheadedness and dizziness, which can lead to an increased likelihood of falls. Patients with $\mathrm{nOH}$ may experience decreased functional ability and an increased fear of falling, which can cause feelings of depression and anxiety, leading to social isolation.

Methods: In a 6-month prospective cohort study of patients with nOH newly initiating droxidopa treatment, participants were asked to quantify the number of good and bad days in the past week. Participants also reported outcomes using other validated instruments that measure nOH symptoms (Orthostatic Hypotension Symptom Assessment Item 1), functional impairment (Sheehan Disability Scale), depressive symptoms (Patient Health Questionnaire-9), fear of falling (Falls Efficacy Scale-International), and health-related quality of life (HRQoL; Short Form-8). Scores after 1 and 6 months of treatment were compared with baseline.

Results: After 1 month of droxidopa treatment, patients reported a significant increase in good days from baseline (mean, 4.2 vs. 3.3 days/week; difference, 0.9 days/week; $\mathrm{P}<0.0001$ ). Similar positive effects for the increase in reported good days were observed at 6 months vs. baseline (mean, 4.5 vs. 3.4 days/week; difference, 1.1 days/week; $\mathrm{P}<0.0001$ ). The significant increase in good days at 1 and 6 months correlated with improvements in other patient-reported outcomes of nOH symptoms, fear of falling, functional impairment, depressive symptoms, and HRQoL (all $\mathrm{P}<0.0001$ ).

Conclusion: Patients treated with droxidopa reported significantly more good days per week compared with before treatment initiation. The increased number of good days tracked with improvements in other patient-reported indicators of mental well-being. Improved 
patient perception of psychological status may represent an important downstream holistic benefit of nOH treatment.

Funding: The data reported were derived from a research project funded by Lundbeck and carried out by Creativ-Ceutical. SK and LAH are employees of Lundbeck. CF is an employee of CreativCeutical, was formerly employed by Lundbeck, and is a shareholder of Lundbeck.

\section{Poster \#33}

\section{Midodrine for the prevention of tilt-induced and clinical vasovagal syncope: A systematic review and meta-analysis}

\author{
L. Lei, S.R. Raj, R.S. Sheldon \\ Libin Cardiovascular Institute, University of Calgary, Calgary, AB, \\ Canada
}

Background: Vasovagal syncope (VVS) is a common clinical problem that significantly reduces quality of life yet lacks effective medical therapies. Existing literature suggests that midodrine might provide effective suppression of recurrent VVS, but studies have used heterogeneous methods and have yielded inconsistent results. The objective of this systematic review was to evaluate the efficacy of midodrine to prevent syncope in patients with recurrent VVS.

Methods: We conducted a systematic search and meta-analysis of studies in MEDLNE, EMBASE, CENTRAL, and CINAHL from database inception to March 2019. We included all randomized controlled trials that compared the effect of midodrine versus control in patients with recurrent VVS. All studies reported a dichotomous outcome of syncope or a predefined syncope surrogate.

Results: Seven studies (320 patients) met criteria for inclusion. The mean study size was 46 (range 12-134) patients. Four studies tested the ability of midodrine to prevent syncope induced by tilttable testing (I2=0\%, 95\% CI: $0-33 \%$ ) and reported that midodrine reduced the likelihood of positive test outcomes (Relative Risk $(\mathrm{RR})=0.37,95 \% \mathrm{CI}: 0.23-0.59, \mathrm{p}<0.001)$. The remaining three studies reported randomized clinical trial results and exhibited a more modest benefit with midodrine ( $R R=0.63,95 \%$ CI: 0.41-0.97, $\mathrm{p}=0.04$ ). However, there was substantial between-study heterogeneity among the three clinical trials ( $\mathrm{I} 2=53 \%$ ), owing in large to the inclusion of one open-label study. The two rigorously performed randomized, placebo-controlled, double-blind studies included 157 subjects with minimal heterogeneity $(\mathrm{I} 2=0)$. These studies reported a much more modest risk reduction with midodrine and only borderline statistical significance ( $R R=0.75,95 \%$ CI: 0.57-1.01, $\mathrm{p}=0.05$ ).

Conclusion: Midodrine is very effective at the prevention of head-uptilt-induced syncope but provides only a $25 \%$ relative risk reduction in double-blinded clinical syncope studies. This modest risk reduction coupled with the drug's requirement for frequent dosing may limit its relevance to the most refractory recurrent VVS patients.

\section{Poster \#34}

\section{Elective EEG monitoring combined with head-up tilt} in the diagnosis of syncope and non-syncopal fainting

J.A.S. Muldowney $3 r d^{1,3}$, P. Sharma ${ }^{1,2}$, L.E. Okamoto ${ }^{1,2}$, H.H. Sonmezturk ${ }^{4}$, A. Peltier ${ }^{4}$, C.A. Shibao ${ }^{1,2}$, I. Biaggioni ${ }^{1,2}$

${ }^{1}$ Department of Medicine, Divisions of ${ }^{2}$ Clinical Pharmacology and ${ }^{3}$ Cardiovascular Medicine, and ${ }^{4}$ Department of Neurology, Vanderbilt University Medical Center, Nashville, Tennessee, USA
Objective: Non-syncopal fainting (NSF) (psychogenic pseudo-syncope) is a diagnostic challenge, leading to unnecessary diagnostic procedures and inappropriate treatments. We explored the utility of elective electroencephalography (EEG) monitoring combined with head-up tilt (HUT) for diagnosis of NSF.

Methods: We reviewed medical records of 131 patients presenting with transient loss of consciousness (TLOC) in whom HUT and EEG were done simultaneously for suspected NSF ( $>20$ lifetime syncopal episodes, $>2$ episodes in a day, $>5$ minutes of LOC). Tilt responses were grouped into three categories: non-diagnostic $(\mathrm{nDg}$, patients with no apparent TLOC or EEG changes during TTT, vasovagal syncope (VVS, TLOC with a slow wave pattern on EEG), and nonsyncopal fainting (NSF, apparent TLOC with normal EEG).

Results: 107 patients were included in the analysis; 57 were $\mathrm{nDg}$ (53\%), 11 had VVS (10\%), and 39 had documented NSF (37\%). There was no difference in age $(42 \pm 16,42 \pm 20$, and $40 \pm 15$ yrs; respectively; $\mathrm{p}=0.736)$ or proportion of female gender $(68,81,78 \%$; respectively; $\mathrm{p}=0.719$, Fisher exact test), but NSF patients were more likely to be taking psychotropic medications. Baseline blood pressure (BP) and heart rate were similar among groups. NSF patients had a shorter time to event ( 9 vs. $19 \mathrm{~min}$. in VVS) and had significantly higher BP during event than those in the $\mathrm{nDg}$ group (systolic $131 \pm 16$ vs. $120 \pm 18 ; \mathrm{p}=0.01$ and diastolic $B P(6 \pm 10$ vs. $80 \pm 12 \mathrm{mmHg}$; $\mathrm{p}=0.05$ ).

Conclusions: In patients with clinical suspicion of NSF, doing EEG monitoring during HUT yields a much higher incidence of NSF (37\%). We suggest that elective EEG monitoring provides a costefficient diagnostic approach, excludes cases of cerebral syncope, and helps in management of patients with NSF. Prospective studies are needed to determine the clinical characteristics that warrant adding EEG monitoring to HUT.

\section{Poster \#35}

In their own words: a qualitative analysis of patients' experience of living with syncope and POTS

M. Runte ${ }^{1}$, T. Runte ${ }^{2}$, S. Jarvie ${ }^{1}$, D. Basil $1^{1}$, S. Bedard ${ }^{3}$, D. Porter ${ }^{1}$, K. Williams-Whitt ${ }^{1}$

${ }^{1}$ Dhillon School of Business and ${ }^{2}$ Faculty of Science, University of Lethbridge, Lethbridge, AB, Canada; ${ }^{3}$ Centre for Excellence on Partnership with Patients and the Public, University of Montreal, Montreal, QC, Canada

Background: There is considerable rhetoric about capturing the 'patient experience', but such research seldom explores how patients actually maneuver their way, not just through the medical system, but also through the complex social context in which they are situated. Further, such research seldom focuses on capturing the experience from the perspective of the patient rather than the health-care providers.

Aims: The overarching goal of this research program is to capture patient experiences of living with a cardiac arrhythmia, compiling a map (or compilation of maps) of the arrhythmia patients' journey and identifying points of crisis or struggle. Recurrent syncope and POTS both have documented deleterious effects on patient quality of life; this study therefore offers a more nuanced perspective on the patient experiences.

Methods: A series of focus groups were conducted with Canadian patients with diagnosed cardiac arrhythmias. Syncope patients and POTS patients with syncope were a subset of the overall data set.

Results: Financial, emotional, social and employment/educational impacts were identified. All patients with recurrent syncope (with or without POTS) experienced significant and sustained quality of life 
impacts. These effects, however, were attenuated in the POTS group. Heightened degrees of ambiguity regarding diagnosis and prognosis, as well as the presence of persisting and challenging symptoms of POTS unrelated to syncope (such as fatigue and brainfog) increased the challenges for POTS patients relative to those patients with syncope without POTS. Findings provide context for understanding the patient experience of living with syncope and POTS, thus facilitating an understanding of the implications of these conditions for life decisions and daily living.

Funding: Cardiac Arrhythmia Network of Canada (CANet) through the Networks of Centres of Excellence.

\section{Poster \#36}

\section{Initial orthostatic hypotension causes (transient) postural tachycardia}

\author{
J.M. Stewart, T. Fialkoff, B. Tuma-Marcella, P. Visintainer, C. \\ Terilli, M.S. Medow \\ Department of Pediatrics and Physiology, New York Medical \\ College, Valhalla, NY, USA
}

Background: Lightheadedness upon rapid standing is common. If systolic/diastolic blood pressure (BP) are reduced by $>40 / 20 \mathrm{mmHg}$ this is denoted "initial orthostatic hypotension" (IOH). History is diagnostic with rapid resolution of hypotension and lightheadedness. Excessive postural tachycardia lasts many seconds confusing IOH with postural tachycardia syndrome (POTS).

Objectives: To test this hypothesis and delimit the range and duration of postural tachycardia in $\mathrm{IOH}$.

Methods: $\mathrm{IOH}$ patients ( $\mathrm{n}=62,16 \pm 3$ years) and healthy volunteers $(\mathrm{n}=15,16 \pm 3$ years) were instrumented for electrocardiography and for beat-to-beat blood pressure. After 10 minutes supine, subjects stood for 5 minutes. After complete supine recovery, subjects underwent $70^{\circ}$-head-up tilt for 10 minutes to rule out POTS.

Results: Baseline data were similar in both groups. BP, HR, and time, referenced to the beginning of standing, were measured at the minimum BP, BP recovery, peak HR, HR minimum and steady state. There were no significant differences in HR when compared at comparable events. BP differed at minimum by design. After BP minimum, events occurred progressively later in $\mathrm{IOH}$ compared to control; Peak HR $=22.5 .0 \pm 0.7$ vs. $15.7 \pm 1.3$; $\mathrm{BP}$ recovery $=$ $26.4 \pm 0.9$ vs. $20.7 \pm 2.5$; HR recovery $=42.8 \pm 3.3$ vs. $27.0 \pm 2.6$; steady state $=89.5 \pm 5.2$ vs. $55.0 \pm 7.8 .87 \%$ of $\mathrm{IOH}$ but not controls had $>30$ s of HR increment $>40 \mathrm{bpm}$.

Conclusions: HR is transiently excessively elevated in $\mathrm{IOH}$, which might be confused with POTS. IOH and control had similar peak HR despite decreased minimum BP in IOH. HR recovery was prolonged in $\mathrm{IOH}$. Data suggest parasympathetic deficiency with blunted cardiovagal baroreflex in $\mathrm{IOH}$.

Funding: NHLBI: RO1HL134674.

\section{Poster \#37}

\section{IVIG for treatment of acquired pseudo-obstruction of unknown etiology}

N. Rademacher, T. Chelimsky, M. Sood, R. Kliegman, D. Basel, G. Chelimsky

Department of Pediatric Gastroenterology, Medical College of Wisconsin, Milwaukee, WI, USA
Patient: 14-year-old female with a complex medical history: severe constipation, recurrent petechiae, sun-sickness, mixed connective tissue disorder, postural tachycardia syndrome (POTS), and hypermobility-Ehlers Danlos syndrome (H-EDS) with Tenascin X deficiency. She was managed at an outside institution with hydroxychloroquine, mycophenolate, and prednisone. Within one month of tapering steroids, the patient developed nausea, vomiting, and abdominal cramping. Gastric emptying scan showed gastroparesis. She received two doses (40 g) IVIG (intravenous immunoglobulin) infusions. In 05/2016 she developed ileus and was transferred to Children's Hospital of Wisconsin. In 06/2016 Mycophenolate was stopped, and in 08/18 a second ileus developed. An antroduodenal motility in 09/2016 showed retrograde propagating small bowel activity and erratic contraction in segments of the small bowel interpreted as nerve etiology. She received an ileostomy due to severe distention and clinical picture compatible with pseudo-obstruction with feeding intolerance. Steroids were restarted.

Tests: Abdominal CT scan, upper endoscopy with enteroscopy, full thickness biopsy of different portions of the GI tract, muscle biopsy, thymidine phosphorylase activity were all normal. Antibodies tested normal. Autonomic testing: Adrenergic vasomotor impairment, with pronounced postural tachycardia and a clear steady drop in blood pressure. Significant rebound pressures upon recline, interpreted as orthostatic hypotension.

Treatments tried: Auricular neurostimulation, cognitive behavioral therapy, octreotide, PT and OT, celiac ganglion block, gabapentin, bethanechol, neostigmine, pyridostigmine, antibiotic regimen, all without improvement. In 02/2018 she received the first dose of highdose IVIG infusions, $2 \mathrm{~g} / \mathrm{kg}$ IVIG over 4 days. This was repeated monthly beginning $04 / 2018$. By $08 / 2018$, the patient tolerated oral feeds and gastric emptying normalized. Repeat antroduodenal motility showed normal motility. She transitioned to all oral feeds. In 05/2019 ileostomy was reversed.

Conclusion: IVIG therapy should be considered in progressive cases of dysmotility. Autoimmune gastrointestinal dysmotility may present without immune markers or histologic findings as reported here, which delayed the use of IVIG.

Funding: Advancing a Healthier Wisconsin, 5520298.

\section{Poster \#38}

\section{Concurrent encephalopathy and autonomic failure in a child with positive ganglionic nicotinic acetylcholine receptor antibodies}

G.A. Cook $^{1}$, J.L. Jaskiewicz ${ }^{2}$

${ }^{1}$ Department of Neurology, Walter Reed National Military Medical Center, Bethesda, MD \& Department of Neurology Uniformed Services University F. Edward Hebert School of Medicine;

${ }^{2}$ Department of Pediatrics, Naval Medical Center, Portsmouth, VA; USA

Objective: Describe a case of autoimmune encephalopathy with accompanying autonomic failure associated with antibodies against the alpha3 subunit of the ganglionic nicotinic acetylcholine receptor. Background: Encephalopathy has rarely been associated with the presence of antibodies against the alpha3 subunit of the gAChR, including one reported pediatric case and one reported adult case associated with antibodies against alpha4 and alpha7 subunits of the gAChR. Further, reversible cognitive impairment has been associated with elevated antibody titers in classic cases of autoimmune autonomic ganglionopathy.

Methods: 14 months prior to presentation, a 12-year-old girl began experiencing difficulty with impaired attention, behavioral outbursts, 
social withdrawal, and insomnia. Her cognitive and behavioral change had been preceded by two months of sore throat and fatigue, diagnosed as infectious mononucleosis. A one-week course or oral corticosteroids five months prior to presentation had temporarily improved her behavior. She had chronic constipation but denied any other autonomic symptoms.

Results: Autonomic testing revealed decreased sudomotor response at the proximal leg with borderline low responses at all other sites (compared to adult-derived norms); decreased heart rate response to deep breathing; prolonged pressure recovery time and blunted phase IV overshoot with Valsalva maneuver; and progressive orthostatic hypotension. Serum testing repeatedly demonstrated mildly elevated antibodies against the alpha3 subunit of the $\mathrm{gAChR}(0.08 \mathrm{nmol} / \mathrm{L}$ on two occasions; normal $<0.02 \mathrm{nmol} / \mathrm{L}$ ). Evaluation of other markers of autoimmunity was negative. CSF analysis, including oligoclonal bands and neopterin, was negative. CSF autoimmune encephalopathy panel (Mayo Laboratories) was normal/negative but did not include gAChR antibody testing. She was treated with intravenous immunoglobulin, after which she had transient improvement of behavioral and cognitive functions.

Conclusions: Antibodies against the alpha3 subunit of the gAChR may be associated with autoimmune encephalopathy. The presence of other associated autoantibodies may play a role in the central nervous system pathology in such cases.

Funding: We are military service members. This work was prepared as part of official duties. Title 17 U.S.C. 105 provides that "Copyright protection under this title is not available for any work of the United States Government." Title 17 U.S.C. 101 defines a United States Government work as a work prepared by a military service member or employee of the United States Government as part of that person's official duties. The views expressed in this article are those of the authors and do not necessarily reflect the official policy or position of the Department of the Navy, Department of Defense, or the United States Government. The authors declare that they have no conflict of interest pertinent to this work. Research data derived from an approved Naval Medical Center, Portsmouth, Virginia IRB, protocol number NMCP.2017.0054.

\section{Poster \#39}

\section{Gastrointestinal (GI) symptoms and GI biomarkers in postural orthostatic tachycardia syndrome (POTS) and cardiogenic syncope (NCS) in adolescents and young adults}

M.T. Numan ${ }^{1}$, D. Halawa ${ }^{2}$, I.J. Butler ${ }^{3}$, H. Zamil ${ }^{2}$ Departments of ${ }^{1}$ Pediatric Cardiology, ${ }^{2}$ Pediatric Gastroenterology and ${ }^{3}$ Pediatric Neurology, University of Texas, Houston, TX, USA

Introduction: Autonomic nervous system supply of gastrointestinal tract (GI) is vast. GI symptoms have been described in the literature related to autonomic dysfunction related to POTS and NCS. This study explores the epidemiology of several GI symptoms in children and young adults presenting with orthostatic intolerance and correlation of these symptoms with serum biomarkers related to the GI system.

Methods: We reviewed our institutional dysautonomia questionnaires for all the patients who presented at Dysautonomia Center of Excellence at The University of Texas, Houston from July 2015 till August 2018. These patients had syncope on head up tilt table test (HUTT) or documented POTS by HUTT. GI symptoms were tabulated as none, mild, moderate and severe according to patients' classification. Blood samples were collected for celiac antigens and antibodies ( $\mathrm{Ab}$ ) including: IgA, Gliadin IgA Ab, Gliadin IgG Ab, tTG $\mathrm{IgA}$ and $\mathrm{tTG} \mathrm{IgG}$, gastric parietal cells $\mathrm{Ab}$, and antinuclear $\mathrm{Ab}$
(ANA). The frequency of these symptoms and presence of these antibodies were correlated.

Results: For this study period, 283 new patients were included. Males were $55(19.4 \%)$ and median age 15.5 years (range 6 to 30 years). Of those 283 patients, $242(85 \%)$ had nausea of which 146 patients reported the nausea as severe $(60 \%)$. Vomiting was present in 136 patients $(48 \%)$ and stomach/intestinal pain was present in 223 patients (79\%). Of those with stomach/intestinal pain, 119 patients reported it as severe $(53.3 \%)$. Gastric parietal cells $\mathrm{Ab}$ were tested in 154 patients which showed positive titer in 16 patients (10.3\%). Celiac antigen and antibodies were tested in 141 patients of which 14 were positive (9.9\%), total IgA positive in 9, Gliadin IgA Ab in 2, Gliadin $\mathrm{IgG} \mathrm{Ab}$ in 3, tTG IgA Ab in 2 and none in tTG IgG Ab. Antinuclear antibodies were tested in 197 patients of which 42 patients $(21.2 \%)$ had elevated titer.

Conclusion: GI symptoms are present in the majority of POTS and NCS patients with nausea as the highest prevalence, followed by stomach/intestinal pain and vomiting, respectively. Serum antibodies and antigens in this population are higher than general population with higher incidences of gastric parietal cells antibodies and ANA titers. We speculate that there is an autoimmune component to POTS/ NCS patients based on our findings

\section{Poster \#40}

\section{Breathing disturbances in a child with Rett syndrome}

H.W. Choi, P. Pianosi

Department of Pediatrics, University of Minnesota, Minneapolis, MN, USA

Background: Rett syndrome (RTT) is a debilitating neurodevelopmental disorder associated with mutations in the methyl $\mathrm{CpG}$ binding protein 2 (MeCP2) gene causing severe cognitive, social, motor and physiological impairments. Autonomic dysregulation is prominent but poorly understood features of this disease.

Case Presentation: A 4-year-old girl with autism spectrum disorder developed breathing abnormalities over several months: intermittent hyperpnea and hyperventilation interspersed with normal breathing, but no discernible apnea while awake. She also had history of acrocyanosis, either spontaneously or in response to cold exposure. Alternatively, sometimes her hands and feet became plethoric. She had long standing history of constipation. Subsequently, the patient was confirmed to be heterozygous for a pathogenic variant in the $\mathrm{MeCP} 2$ gene, which is consistent with the diagnosis of MeCP2-related disorder.

Discussion: Here, we present a unique case of a child with RTT who manifests various symptoms of autonomic dysregulation. Autonomic dysregulation in RTT can give rise to impairments occurring in multiple physiological systems. In our patient, breathing rhythm abnormality was the diagnostic cue for Rett's syndrome. Julu et al. described 3 respiratory phenotypes : phenotype 1 are forceful breathers who hyperventilate causing hypocapnia and chronic respiratory alkalosis; phenotype 2, so-called "feeble breathers", typically hypoventilate resulting in hypercapnia and chronic respiratory acidosis; phenotype 3 display apneustic breathing - gasping inhalations with a breath-hold before exhalation. This pattern is grossly irregular and may be accompanied by lengthy periods of apnea, resulting in $\mathrm{CO}_{2}$ retention due to delayed, insufficient exhalations. Blood gases were not measured in our patient but our patient was most likely phenotype 1 . Such characterization may lead to options for treatment, if indicated. Unraveling the autonomic component of respiratory and behavioral dysregulation would be critical in developing treatment strategies and translationally relevant outcome measures. 


\section{Poster \#41}

\section{Postural tachycardia syndrome in a family with pseudoxanthoma} elasticum

\author{
A. Arvantaj, C. Geiger, J. Robinson, B. Katirji \\ Neurological Institute, University Hospitals Cleveland Medical \\ Centre, Cleveland, OH, USA
}

Introduction: There is a wealth of evidence showing correlation between hypermobility syndromes (Ehlers Danlos syndrome as the prototype) and autonomic dysregulations including postural tachycardia syndrome (POTS). Pseudoxanthoma elasticum (PXE) is a less common hypermobility disorder involving the skin, eyes and cardiovascular system. A subtype of PXE is associated with vitamin $\mathrm{K}$-dependent coagulation factor deficiency. It results from a mutation in GGCX (gamma-glutamyl carboxylase) gene which activates both vitamin K-dependent coagulation factors (II, VII, IX and X) and matrix Gla protein (MGP). MGP is an inhibitor of ectopic mineralization in peripheral connective tissues. To our knowledge this is the first report of association between PXE and dysautonomia.

Materials: A family of three (mother and her two daughters) were diagnosed with POTS based on clinical symptoms and positive tilt table test (symptomatic rise in heart rate of more than 40 beats per minute). The mother and one of the daughters had a mutation on GGCX gene. All three family members had vitamin $\mathrm{K}$ dependent coagulation factors deficiency (taking vitamin $\mathrm{K}$ supplement), skin involvement (large plaques of inelastic, leathery and loose skin with yellowish hue), joint (subluxations) and hypermobility (high Beighton score).

Conclusion: This study of POTS in a family with pseudoxanthoma elasticum and GGCX gene mutation contributes by adding this disorder to the list of hypermobility syndromes associated with dysautonomia. Understanding the pathophysiology of PXE (specifically GGCX subtype) may be helpful to understand the casual correlation of hypermobility syndromes and dysautonomia.

\section{Poster \#42}

\section{Synergistic but separable sensory changes in migraineurs and postural tachycardia syndrome}

M.M. Cortez, L. Millsap, K.C. Brennan Department of Neurology, University of Utah, Salt Lake City, UT, USA

Background: Up to $90 \%$ of postural tachycardia syndrome (POTS) patients report frequent, problematic headaches. According to current diagnostic criteria for POTS, associated headaches might be expected to be strictly orthostatic, though comorbid migraine headache is common. Given this observation, it is possible that the two disorders interact pathophysiologically.

Methods: 30 POTS, 30 chronic migraine (CM), and 30 non-headache (NH) control subjects completed a migraine questionnaire, MIDAS/ HIT6, and sensory/autonomic symptom assessment. Sensory testing included mechanical pain thresholds by von Frey hair and quantitative light sensitivity thresholds.

Results: Photophobia and allodynia symptom scores were significantly higher in both $\mathrm{CM}$ and POTS groups compared to controls. COMPASS-31 autonomic symptom scores were significantly higher in POTS compared to $\mathrm{CM}$, but both groups had significantly higher scores than NH. Unexpectedly, photophobia thresholds were significantly lower in POTS compared to CM; both POTS and CM thresholds were significantly decreased compared to $\mathrm{NH}$. Mechanical pain thresholds were also significantly lower in both CM and POTS compared to $\mathrm{NH}$. Here the patterns of expression differed between $\mathrm{CM}$ and POTS, with reduced periorbital and forearm pain thresholds in CM, and only forearm threshold reductions in POTS subjects. Discussion: We found evidence of 'migraine-like' sensitization and autonomic symptoms in both CM and POTS subjects, suggesting common pathway activation in these often-comorbid conditions. However, we also observed distinguishable features in each disorder that likely reflect divergent network responses. We found that POTS subjects (including POTS subjects without migraine) had significantly lower light sensitivity thresholds, and a differing pattern of allodynia, compared to CM. This reveals an under-appreciated aspect of disease burden in POTS, and suggests network sensitization similar to, but separable from that of migraine.

\section{Poster \#43}

Body compression in postural tachycardia syndrome improves orthostatic tolerance in a dose-dependent pattern

K.M. Bourne, R.S. Sheldon, D.V. Exner, J. Tyberg, K. Kogut, J. Ng, N. Sheikh, M. Lloyd, E. Scott, S.R. Raj

University of Calgary, Calgary, AB, Canada

Background: Postural tachycardia syndrome (POTS) is a common form of orthostatic intolerance. Compression garments are frequently prescribed, but little evidence exists to validate their efficacy in this disorder.

Objective: To evaluate the effects of a lower body compression garment in different configurations on orthostatic tolerance and symptom rating in POTS.

Methods: Participants completed 4x10 min $80^{\circ}$ head up tilts (HUTs) in one study session. A non-inflatable compression garment was used in 4 configurations: full (FC), none (NC), abdomen/upper leg ( $\mathrm{AC}$ ) and lower leg (LC). Each participant experienced the 4 conditions in a cross-over, randomized-order study with each participant serving as their own controls. ECG was recorded and beat-to-beat blood pressure was measured. Symptoms were assessed using the Vanderbilt Orthostatic Symptom Score (VOSS; Range 0-90). Data are presented as mean \pm SEM.

Results: This study is currently enrolling and 20 POTS patients $(\mathrm{n}=18$ female, age: $30 \pm 2$ year; BMI: $23 \pm 1 \mathrm{~kg} / \mathrm{m}^{2}$ ) are included in this preliminary analysis. The mean increase in HR across the $10 \mathrm{~min}$ HUT (compared to $10 \mathrm{~min}$ baseline) followed a dose-dependent pattern, with NC ( $35 \pm 3 \mathrm{BPM})$, producing the highest HR increase, followed by LC ( $30 \pm 3$ BPM), AC ( $25 \pm 2$ BPM), and FC $(20 \pm 2$ $\mathrm{BPM}$ ), $\mathrm{p}<0.001$. Orthostatic Index (area under curve:RR interval versus time) was largest in $\mathrm{FC}(6.7 \pm 0.2 \mathrm{~min} / \mathrm{Hz})$, followed by $\mathrm{AC}$ $(6.4 \pm 0.2 \mathrm{~min} / \mathrm{Hz}), \mathrm{LC}(5.9 \pm 0.2 \mathrm{~min} / \mathrm{Hz})$ and $\mathrm{NC}(5.8 \pm 0.2 \mathrm{~min} / \mathrm{Hz})$, $\mathrm{p}<0.001$. Mean systolic blood pressure (SBP) decrease on HUT followed the same dose-dependent pattern with $\mathrm{NC}(11 \pm 2 \mathrm{mmHg})$, followed by LC $(10 \pm 1 \mathrm{mmHg}), \mathrm{AC}(8 \pm 1 \mathrm{mmHg})$ and $\mathrm{FC}(6 \pm 1$ $\mathrm{mmHg}), \mathrm{p}=0.004$, but all changes were within diagnostic criteria $(<20$ $\mathrm{mmHg})$. Stroke volume decreased less on HUT with FC $(24 \pm 2 \mathrm{~mL})$ and $\mathrm{AC}(24 \pm 2 \mathrm{~mL})$ than $\mathrm{LC}(33 \pm 3 \mathrm{~mL})$ and $\mathrm{NC}(34 \pm 2 \mathrm{~mL})$, $\mathrm{p}<0.001$. VOSS symptom scores also followed a dose-dependent trend, with lowest scores reported with FC (14 \pm 2$)$, followed by AC $(15 \pm 3), \mathrm{LC}(21 \pm 4)$, and NC $(25 \pm 4), \mathrm{p}=0.005$.

Conclusion: Lower-body compression decreased HR, reduced symptoms, and maintained SBP during HUT, in a dose-dependent pattern. Stroke volume was better maintained on HUT with full and abdominal compression. 


\section{Poster \#44}

Diagnosis of postural tachycardia syndrome: a comparison between the tilt and active stand tests

V. Galvis, A.I. Penzlin, B. Roschach, S. Rajan, J. Celli, I. Bonyhay, R. Freeman, C.H. Gibbons

Department of Neurology, Beth Israel Deaconess Medical Center, Harvard Medical School, Boston, MA, USA

Background: Postural tachycardia syndrome (POTS) is defined by a sustained heart rate increase of $\geq 30$ beats/minute within 10 minutes of upright tilt or active standing in the absence of orthostatic hypotension.

Objective: To determine the differential utility of independent tilt and active standing tests in the diagnosis of POTS.

Methods: We reviewed the records of 50 patients (mean age 32, range 15-53) who were referred for autonomic testing. Testing included heart rate variability to paced breathing, Valsalva maneuver, a 45 minute tilt table test and a 5 minute active stand with continuous heart rate and beat-to-beat blood pressures, and automated sphygmomanometer recorded each minute of testing. Groups were analyzed by diagnosis of POTS on tilt alone, stand alone or with both tests. Results were correlated with other autonomic function testing.

Results: 35 patients were diagnosed with POTS by both tests, 11 patients were diagnosed with standing alone and 4 patients were diagnosed with tilt table test alone. The group with POTS diagnosed by tilt alone had higher SBP $(\mathrm{P}<0.01$, ANOVA) and DBP $(\mathrm{P}<0.01$ ANOVA) compared to those diagnosed with POTS by stand alone or tilt and stand. There were significant differences in heart rates between groups: on tilt alone heart rate was $97 \pm 16$ BPM, on stand alone the HR was $115 \pm 11 \mathrm{BPM}$, with tilt and stand the heart rate was $122 \pm 17$ BPM $(\mathrm{P}<0.01$ ANOVA). There was no difference between these groups and other autonomic tests $(\mathrm{P}=0.606)$.

Discussion: Although a diagnosis of postural tachycardia can be made with tilt or active stand tests, we found significant differences in both heart rate changes and blood pressure between the identified groups which suggest that both tests are independently necessary for accurate diagnosis and assessment of POTS. These data suggest that different presenting symptoms and pathophysiologic mechanisms may underlie the syndrome in these groups.

\section{Poster \#45}

Cognitive function is impaired during active standing in postural orthostatic tachycardia syndrome

A.J. Miller ${ }^{1}$, T. Sheehan ${ }^{2}$, M. Feeley ${ }^{1}$, K. Bourne ${ }^{3}$, A.C. Arnold ${ }^{1}$ ${ }^{1}$ Department of Neural and Behavioral Sciences, Penn State College of Medicine, Hershey, PA, USA; ${ }^{2}$ Department of Neurology, Medical University of South Carolina, Charleston, SC, USA; ${ }^{3}$ Department of Cardiac Sciences, University of Calgary School of Medicine, Calgary, $\mathrm{AB}$, Canada

Introduction: Cognitive dysfunction or "brain fog" is commonly observed in patients with postural tachycardia syndrome (POTS). To our knowledge, no studies have objectively measured cognition in POTS during active standing when patients are most symptomatic in daily life. We hypothesized cognitive performance would be impaired in POTS during active standing.

Methods: Eighty-four POTS patients (94\% female, $29 \pm 11$ years, $15 \pm 3$ education years) and 18 healthy controls (90\% female, $31 \pm 13$ years, $16 \pm 1$ education years) completed cognitive testing in the supine and active standing positions in a randomized crossover design. The tests included Stroop word-color test of executive function and Cogstate Identification task (IDN) to measure attention. Stroop word-color scores were normalized based on age and education level and the IDN average speed (seconds) was calculated with higher numbers indicating worse performance. Data were analyzed by two-factor ANOVA group (POTS, control) and posture (supine, standing) with post-hoc Tukey's tests.

Results: Stroop word-color scores were not affected by posture $(p=0.984)$, but tended to be lower in POTS compared to controls $(p=0.109)$. There was an interaction for IDN scores $(p=0.031)$ with significant group $(\mathrm{p}<0.001)$ but not posture $(\mathrm{p}=0.345)$ main effects. IDN scores were worsened by standing in POTS (supine: $2.73 \pm 0.010$, standing: $2.78 \pm 0.01, \mathrm{p}=0.001)$ but not in controls $(\mathrm{p}=0.908)$. POTS patients had slower IDN scores compared to controls in the standing posture (POTS: $2.78 \pm 0.01$, Controls: $2.68 \pm 0.01, \mathrm{p}=0.001$ ) suggesting impaired attention, with no group differences in the supine posture (POTS: $2.73 \pm 0.01$, Controls: $2.70 \pm 0.01, \mathrm{p}=0.571$ ).

Discussion: POTS patients had significant inattention and a trend towards decreased executive function compared to controls. Attention was worsened by standing in POTS but not in controls. These data indicate selective impairment in the attention domain of cognition in POTS during active standing which may contribute to "brain fog". Funding: Dysautonomia International.

\section{Poster \#46}

Prevalence of hypermobile Ehlers-Danlos syndrome in postural orthostatic tachycardia syndrome

A.J. Miller ${ }^{1}$, L.E. Stiles ${ }^{2,3}$, T. Sheehan ${ }^{4}$, R. Bascom ${ }^{5}$, H.P. Levy ${ }^{6}$, C. Francomano ${ }^{7}$, A.C. Arnold ${ }^{1}$

${ }^{1}$ Department of Neural and Behavioral Sciences, Penn State College of Medicine, Hershey, PA, USA; ${ }^{2}$ Department of Neurology, Stony Brook University School of Medicine, Stony Brook, NY, USA; ${ }^{3}$ Dysautonomia International, East Moriches, NY, USA; ${ }^{4}$ Department of Neurology, Medical University of South Carolina, Charleston, SC, USA; ${ }^{5}$ Department of Medicine, Penn State College of Medicine, Hershey, PA, USA; ${ }^{6}$ Department of Medicine and McKusick-Nathans Institute of Genetic Medicine, Johns Hopkins School of Medicine, Baltimore, MD, USA; ${ }^{7}$ The Harvey Institute for Human Genetics, Greater Baltimore Medical Center, Baltimore, MD, USA

Introduction: Hypermobile Ehlers-Danlos syndrome (hEDS) is an inherited connective tissue disorder characterized by joint hypermobility. Previous studies found that approximately $30 \%$ of patients with postural tachycardia syndrome (POTS) have EDS. However, these studies were on small single-center cohorts, relied on self-report, or did not specify EDS subtype. Furthermore, the diagnostic criteria for hEDS were updated in 2017. The goal of the present study was to investigate the prevalence of hEDS using the current diagnostic criteria in a convenience sample of POTS patients.

Methods: POTS participants were recruited at the 2018 Dysautonomia International conference. Ninety-one POTS participants 13-60 years (average: $29 \pm 1$ ) completed orthostatic vitals and evaluation using the 2017 hEDS diagnostic classification, which has three major criteria: 1) generalized joint hypermobility (Beighton score), 2) systemic features, family history, and complications of joint hypermobility, 3) exclusion criteria.

Results: Overall, 28 participants (31\%) qualified for hEDS by satisfying all three criteria. Fifty participants (54\%) met criterion 1 based on the Beighton score cutoffs for their age. Criterion 2 was fulfilled by 37 participants (41\%). Only 6 participants $(7 \%)$ had a positive family history of hEDS. Criterion 3 was fulfilled by 77 participants $(86 \%)$. 
Discussion: This is the first study to evaluate the prevalence of hEDS in a large sample of POTS participants using the updated hEDS diagnostic criteria. Overall, $31 \%$ of POTS participants were classified as having hEDS based on the 2017 criteria, which is consistent with previous estimates. In most of these cases no first degree relatives were previously diagnosed with hEDS. Half of participants were hypermobile indicating high prevalence of hypermobility spectrum disorders. Identifying the prevalence of hEDS in POTS is an important step in understanding the relationship between these two conditions.

Funding: Dysautonomia International.

\section{Poster \#47}

Immunotherapy as an effective treatment of POTS with functionally active adrenergic and muscarinic antibodies after human papillomavirus vaccination: a case report

A.T. Del Pozzi ${ }^{1}$, G. Wallukat ${ }^{2}$, I. Schimke ${ }^{2}$, S. Blitshteyn ${ }^{3}$

${ }^{1}$ Integrative Exercise Physiology Laboratory, Ball State University, Muncie, IN, USA; ${ }^{2}$ Berlin Cures GmbH, Berlin, Germany;

${ }^{3}$ Dysautonomia Clinic, Jacobs School of Medicine and Biomedical Sciences, Buffalo, NY, USA

Objective: We describe clinical presentation, diagnostic studies and response to treatment of an 18-year-old woman who developed POTS after a bivalent HPV vaccine, Cervarix, with positive serum beta- 2 adrenergic and M2-muscarinic antibodies.

Methods and Results: Autonomic function tests showed postural tachycardia on a tilt table test and unremarkable Valsalva, deep breathing, QSART and skin biopsy. Cerebrospinal fluid analysis showed no evidence of infection, inflammation or demyelination. Extensive evaluation for defined systemic and neurologic autoimmune disorders was negative. Using a bioassay (Berlin Cures $\mathrm{GmbH}$ ), functionally active anti-beta- 2 adrenergic $(\beta 2-\mathrm{AAB})$ and anti-muscarinic receptor 2 antibodies (M2-AAB) were detected in the serum of the patient. Clinically, she was home or bed-bound with extreme fatigue, dizziness, postural tachycardia, nausea, and cognitive dysfunction for 5 years despite utilizing various medications and nonpharmacotherapy for POTS. Almost complete resolution of symptoms with the patient improving from being bed-bound to working fulltime and running a 5 kilometer race was achieved with the use of immunotherapy consisting first of plasmapheresis and subsequently, of intravenous immunoglobulin and prednisolone. Symptoms re-appeared after immunotherapy was held, and disappeared with the reinstitution of immunotherapy. Symptomatic improvement correlated with decreased titers of $\beta 2-\mathrm{AAB}$ to the lower range of healthy individuals and undetectable titers of M2-AAB

Conclusion: This case illustrates several important points: 1) Postvaccination POTS is likely an autoimmune phenomenon. 2) Immunotherapy can be an effective treatment of post-vaccination POTS. 3) Functionally active adrenergic and muscarinic antibodies appear to be significant, and possibly causative, in our patient. 4) Commercially-available testing for the adrenergic and muscarinic antibodies is needed in the United States. 5) Serum adrenergic and muscarinic antibodies may be potential objective markers for the diagnosis, symptom severity, and response to treatment in patients with POTS. Further research is necessary to study these antibodies in patients with POTS, including in those with post-vaccination onset.

\section{Poster \#48}

\section{Natural history of postural tachycardia syndrome}

A.I. Penzlin, B. Roschach, A. Benson, V. Galvis, I. Bonyhay, C.H. Gibbons, R. Freeman

Department of Neurology, Beth Israel Deaconess Medical Center, Boston, MA, USA

Background: Postural tachycardia syndrome (POTS) is a chronic condition characterized by an exaggerated increase in upright heart rate with associated symptoms. The long-term outcome and factors associated with the outcome are not well elucidated.

Objective: To define the long-term outcome and associated factors in patients with POTS.

Methods: Patients fulfilling diagnostic criteria for POTS completed questionnaires several years after diagnosis. Questionnaires included a survey of 22 open and closed-ended questions assessing symptoms, daily activities, quality of health, and treatments. Patients rated symptom severity when at their worst and at follow up (FU) on a 0 to 10 scale. Patients whose score decreased by $8-10$ pts between their worst and FU were considered much improved, while a smaller decrease (1-7 pts) was considered somewhat improved. Relationships between degree of improvement and variables that may have influenced disease course were analyzed.

Results: Fifty-five POTS patients $(37.26 \pm 12.38$ years, $83.7 \%$ female $)$ were studied. Average time from diagnosis to FU was $6.3 \pm 2.9$ years. Almost all participants were less symptomatic at FU: $3.6 \%$ kept the same score, $34.5 \%$ improved by $1-3$ pts, $30.9 \%$ by $4-5$ pts, $20 \%$ by 6-7 pts, and $10.9 \%$ by $8-10$ pts. Time between diagnosis and FU did not differ among the groups $(\mathrm{p}=\mathrm{NS})$. Antecedent infection was reported by $80 \%$ of much improved patients (vs. $26.1 \%$ somewhat improved, $\mathrm{p}=0.04$ ) and stress by $40 \%$ (vs. $17.4 \%, \mathrm{p}=0.5$ ). Among much improved patients, $66.7 \%$ reported a substantial increase in exercise (vs. $21.3 \%, \mathrm{p}=0.04$ ). Diet changes were adopted by $33.3 \%$ of much improved patients (vs. 59.6\%, p=NS). Gluten free diet was the most frequently adopted dietary change. The majority of much improved patients attributed their convalescence to medication (66.7\% vs. $25.5 \%, \mathrm{p}=0.17)$ and treatment $(100 \%$ vs. $43.5 \%, \mathrm{p}=0.6)$. Discussion: Our findings show that most POTS patients improve over time. Improvement is associated with self-reported preceding infection and increased physical activity. Disease duration is not a predictor of improvement.

\section{Poster \#49}

Effects of transcutaneous vagal nerve stimulation on orthostatic tolerance in patients with postural tachycardia syndrome (POTS)

D. Shiffer ${ }^{1}$, R. Furlan ${ }^{1}$, F. Barbic ${ }^{1}$, M. Minonzio ${ }^{1}$, B. Cairo ${ }^{2}$, A. Porta ${ }^{2,3}$, N. Montano ${ }^{4}$, E. Tobaldini ${ }^{4}$, L. Furlan ${ }^{4}$, V. Urechie ${ }^{1,5}$, I. Biaggioni ${ }^{5}$, A. Diedrich ${ }^{5}$

${ }^{1}$ Internal Medicine, Humanitas Clinical and Research Center-IRCCS; Department of Biomedical Sciences, Humanitas University, Rozzano, Milan, Italy; ${ }^{2}$ Department of Biomedical Sciences for Health, University of Milan, Milan, Italy; ${ }^{3}$ Department of Cardiothoracic, Vascular Anesthesia and Intensive Care, IRCCS Policlinico San Donato, San Donato Milanese, Milan, Italy; ${ }^{4}$ Internal Medicine, Allergology \& Immunology, Ospedale Maggiore Policlinico; IRCCSCà Granda Foundation, University of Milan, Milan, Italy; ${ }^{5}$ Autonomic Dysfunction Center, Vanderbilt University, Nashville, TN, USA

Background: In postural tachycardia syndrome (POTS) many symptoms and signs may be related to a cardiac sympathetic over-activity. 
An increase of cardiac vagal modulation and/or decrease of cardiovascular sympathetic drive might result in clinical improvement of POTS. Transcutaneous vagal nerve stimulation (tVNS) proved to be effective in increasing cardiac vagal modulation.

Aim: To evaluate whether tVNS might reduce orthostatic symptoms and change hemodynamic parameters during orthostasis in POTS.

Methods: Nine female POTS patients $(35 \pm 14$ years; BMI $21.2 \pm 4.2$ $\mathrm{kg} / \mathrm{m}^{2}$ ), of possible hyperadrenergic phenotype [upright: norepinephrine $>600 \mathrm{pg} / \mathrm{ml}$, underwent continuous ECG, BP and respiratory activity recordings in supine position and during a $75^{\circ}$ head-up tilt, during sham stimulation and tVNS. tVNS was administered by a noninvasive, battery-powered device (NEMOS; Cerbomed, Germany). Electrical stimulation was delivered through the skin at the right ear concha (pulse width $200 \mu$ s; frequency $25 \mathrm{~Hz}$ ) while supine and during tilt. Stimulation amplitude was individually adjusted to maximal tolerable level $(1.8 \pm 0.5 \mathrm{~mA})$ or zero (sham). A modified Vanderbilt Orthostatic Symptoms Scale was used to quantify symptoms (mental clouding, blurred vision, short of breath, rapid heartbeat, tremulousness, chest discomfort, headache, lightheaded, nausea) in a range of zero (no symptom) to 10 (maximum intensity). Results: During tVNS, the increase of heart rate (HR) induced by tilt was lower $(\Delta 30.0 \pm 9.2$ from $77.6 \pm 11.2 \mathrm{~b} / \mathrm{min} ; \mathrm{p}<0.05)$ than during sham stimulation $(\Delta 35.1 \pm 9.5$ from $75.6 \pm 11.0 \mathrm{~b} / \mathrm{min})$. Upright arterial pressure and respiratory activity were unchanged during sham stimulation and tVNS. During tilt, symptoms intensity was lower $(\mathrm{p}<0.05)$ during tVNS $(3.3 \pm 0.9)$ compared to sham stimulation $(6.0 \pm 1)$.

Conclusion: tVNS seems to improve overall symptoms intensity and reduce the magnitude of HR increase during the orthostatic stimulus in hyperadrenergic POTS patients.

\section{Poster \#50}

\section{A double-blind, placebo-controlled, crossover pilot trial of gabapentin for treatment of postural tachycardia symptoms}

R.K. Khurana

Department of Internal Medicine, MedStar Union Memorial Hospital, Baltimore, MD, USA

Background: Few evidence-based treatments exist for postural tachycardia syndrome (POTS), a heterogeneous disorder that presents with multiple disabling symptoms including orthostatic intolerance, "panic," fatigue, palpitations, insomnia, and photophobia. Gabapentin diminishes rectal mechanosensitivity, enhances slow-wave sleep, and reduces anxiety, but has not been studied in POTS.

Objective: To study the therapeutic potential of gabapentin in curtailing POTS symptoms in an IRB-approved, prospective doubleblind crossover comparison with placebo.

Methods: Ten POTS patients participated, 9 women, mean age 31 years, symptom duration $>1$ year, mean orthostatic heart rate increase 42 beats/min, 6 with neuropathic sweat pattern. Gabapentin and placebo administration was randomized and double-blinded. Treatment progressed from $100 \mathrm{mg}$ at night to $300 \mathrm{mg}$ twice daily over 6 days with post-trial data obtained on day 7 after 300-mg treatment. A 1 -week washout period was observed between treatments. Outcome measures were acute panic inventory, somatosensory amplification scale, insomnia severity index, palpitation awareness severity score when resting and after the Valsalva maneuver, and photosensitivity index. Linear regression was used to test carryover effects, and t test/ Wilcoxon rank sum test was used to compare gabapentin and placebo data. Baseline and first treatment were compared where significant crossover effect was found.
Results: No significant carryover effects were found in 10 of the 12 outcomes measured. No significant differences were found between gabapentin and placebo treatment or between baseline and first treatment for any of the outcome criteria. Six patients preferred to continue gabapentin treatment post-trial at a daily dose of 200-1200 $\mathrm{mg}$. These patients subjectively reported $50 \%-75 \%$ improvement in insomnia, palpitations, fatigue, and brain fog.

Conclusions: Gabapentin provided no objective benefit. Subjective benefit was reported by six of ten patients. This dichotomy may indicate a large placebo effect. Further confirmation by a large-scale study may be warranted.

\section{Poster \#51}

Cerebral blood flow, autoregulation, and symptoms during orthostasis with lower body compression in postural tachycardia syndrome

M.G. Lloyd, K.M. Bourne, R.S. Sheldon, D.V. Exner, J. Tyberg, K. Kogut, J. Ng, N. Sheikh, E. Scott, S.R. Raj

Libin Cardiovascular Institute of Alberta, University of Calgary, Calgary, AB, Canada

Background: Previous research indicates that orthostatic symptoms in postural tachycardia syndrome (POTS) are due in part to larger blood pressure variability while upright, with concomitant increased cerebral blood flow variability due to reduced cerebral autoregulation. To date, no studies have assessed the effect of any treatment on blood pressure variability and cerebral autoregulation. Static lower body compression garments are commonly prescribed to POTS patients, but their effects on cerebral blood flow mechanics and blood pressure variability are unknown. We hypothesized that lower body compression would reduce blood pressure variability, and improve cerebral autoregulation and orthostatic symptoms.

Objective: To evaluate the brain blood flow and cerebral autoregulation mechanics in POTS patients during orthostasis with and without a static compression garment.

Methods: Participants completed two sequential 10-minute head-up tilts (HUTs). No compression (NC) and full compression (FC) were applied in a random order. Beat-to-beat blood pressure, heart rate (ECG, lead aVF), and cerebral blood flow velocity (CBFV) of the middle cerebral artery were measured. Cerebral autoregulation was calculated using the transfer function coherence between mean arterial pressure (MAP) and CBFV. Orthostatic symptoms were assessed at the end of each 10 min tilt using the Vanderbilt Orthostatic Symptom Score (VOSS, range 0-90). Data are represented as mean \pm standard error.

Results: This preliminary analysis includes 20 POTS patients $(\mathrm{n}=18$ female, age $30 \pm 2$ years, $B M I=23 \pm 1 \mathrm{~kg} / \mathrm{m}^{2}$ ). Low-frequency systolic blood pressure variability was greater during $\mathrm{NC}\left(41 \pm 7 \mathrm{mmHg}^{2} / \mathrm{Hz}\right)$ than FC $\left(17 \pm 7 \mathrm{mmHg}^{2} / \mathrm{Hz}, \mathrm{p}=0.02\right)$. During HUT, CBFV was not different between $\mathrm{NC}(44 \pm 4 \mathrm{~cm} / \mathrm{s})$ and $\mathrm{FC}(49 \pm 4 \mathrm{~cm} / \mathrm{s}, \mathrm{p}=0.5)$. MAP:CBFV coherence was greater during $\mathrm{NC}(0.87 \pm 0.06)$ than FC $(0.69 \pm 0.06, \mathrm{p}=0.02)$, indicating improved autoregulation during $\mathrm{FC}$. During HUT, VOSS was greater during NC $(25 \pm 3)$ than FC $(13 \pm 3$, $\mathrm{p}=0.0001$ ).

Conclusion: Lower-body compression does not affect CBFV, but reduces low-frequency systolic blood pressure variability, improves dynamic cerebral autoregulation and orthostatic symptoms in POTS patients.

Funding: This work was funded by grants from CIHR and the Libin Cardiovascular Institute of Alberta. 


\section{Poster \#52}

Norepinephrine transporter inhibition with atomoxetine: autonomic effects during orthostasis in patients with vasovagal syncope

M.G. Lloyd, L.Lei, J.C. Guzman, T. Kus, F.A. Ayala-Paredes, R.S. Sheldon, S.R. Raj

University of Calgary, Calgary, AB, Canada

Background: Norepinephrine transporter inhibition with atomoxetine reduces head-up tilt (HUT) induced fainting in vasovagal syncope (VVS). However, the effects on the autonomic responses to orthostasis are unknown.

Objective: To investigate the effect of atomoxetine on the hemodynamic and autonomic responses to orthostasis. We hypothesized that atomoxetine would increase circulating norepinephrine, vascular resistance, and sympathetic vascular tone during orthostasis in patients with VVS.

Methods: VVS patients were randomized (double-blind) to receive $2 \times 40 \mathrm{mg}$ doses of atomoxetine (ATOM) or placebo (PL). Heart rate (HR) and beat-to-beat blood pressure were recorded during a 10-minute baseline, and 80-degree HUT for up to 60 minutes. Circulating venous norepinephrine was collected during baseline and at 10 minutes following HUT. Spontaneous baroreflex sensitivity (BRS), heart rate variability (HRV) and blood pressure variability (BPV) were calculated while supine and during HUT. Data are presented as mean \pm SE.

Results: We randomized 56 patients (39 F; $37 \pm 2$ years) with VVS. Systemic vascular resistance increased during HUT within both groups $(\mathrm{p}=0.0001)$, but no group differences were detected (ATOM: $+1.2 \pm 0.5 \mathrm{mmHg} \cdot \mathrm{min} / \mathrm{L}$, PL: $+1.4 \pm 0.4 \mathrm{mmHg} \cdot \mathrm{min} / \mathrm{L}, \mathrm{p}=0.57)$. Circulating norepinephrine increased during HUT in both groups $(\mathrm{p}<0.0001)$, but no group differences were detected (ATOM: $+283 \pm 38 \mathrm{pg} / \mathrm{mL}$, PL: $+243 \pm 37 \mathrm{pg} / \mathrm{mL}, \mathrm{p}=0.46)$. Low-frequency BPV increased in both groups during HUT $(\mathrm{p}<0.001)$, but was not different between groups (ATOM: $+12 \pm 3 \mathrm{mmHg}^{2}$ vs. $+10 \pm 3$ $\left.\mathrm{mmHg}^{2}, \mathrm{p}=0.8\right)$. The increase in HR upon HUT was larger with ATOM than PL $(+29 \pm 3$ bpm vs. $+18 \pm 3$ bpm, $p=0.01)$. Resting BRS was higher in the ATOM group $(20 \pm 2 \mathrm{~ms} / \mathrm{mmHg}$ vs. $14 \pm 2 \mathrm{~ms} /$ $\mathrm{mmHg}, \mathrm{p}=0.046)$, and BRS decreased more during HUT $(-14 \pm 2 \mathrm{~ms} /$ $\mathrm{mmHg}$ vs. $-8 \pm 2 \mathrm{~ms} / \mathrm{mmHg}, \mathrm{p}=0.05)$. High-frequency HRV decreased upon HUT in both groups (each $\mathrm{p}<0.001$ ), but was not different between groups (ATOM: $-419 \pm 103 \mathrm{~ms}^{2}$ vs. PL: $-473 \pm 110 \mathrm{~ms}^{2}$, $\mathrm{p}=0.6$ ).

Conclusion: Atomoxetine increased resting BRS, allowing for augmented BRS decrease and HR responses during HUT. Vascular resistance, circulating norepinephrine, and low-frequency BPV were unaffected by atomoxetine in therapeutic doses.

Funding: Grant \#15-P01-001 from the Cardiac Arrhythmia Network of Canada (CANet).
Thursday, November 7, 2019

\section{POSTER SESSION II}

\section{Poster \#53}

Cannabis induces changes in functional brain connectivity that correlate with increased vagal tone and clinical analgesia in chronic neuropathic pain

H. Sharon, L. Weizman, J. Espaniol, L. Dayan, G. Jacob Medicine and Recanati Autonomic Dysfunction Center and Sagol Brain Institution and Pain Medicine, Tel Aviv Sourasky Medical Center, Sackler Faculty of Medicine, TAU, Tel Aviv, Israel

Objectives: The neural mechanisms underlying the analgesic effect of $\delta$-9-tetrahydrocannabinol (THC) remain incompletely understood. Since some reports revealed that the vagal nerve plays a role in pain modulation, we aimed to study the effect of THC on vagal tone and conditioned pain modulation (CPM) in patients with chronic neuropathic pain. Additionally, we used fMRI to examine the functional connectivity in brain areas relevant to pain processing and autonomic function.

Methods: Thirteen patients with chronic neuropathic radicular pain participated in a randomized, double-blind, placebo-controlled trial employing a crossover design. Heart rate variability, pain scores and CPM were assessed at baseline and after administration of either sublingual THC or placebo. We also examined the functional connectivity of the dorsolateral prefrontal cortex (DLPFC) and the anterior cingulate cortex (ACC), major cognitive-emotional brain hubs, with the anterior insula, hippocampus and rostro ventro-lateral medulla (RVLM).

Results: Compared to placebo, THC caused a significant reduction in spontaneous pain ratings $(\mathrm{p}=0.020)$, and improved CPM measures $(\mathrm{p}=0.048)$. The HFn and LF/HF ratio of the RR intervals were comparable at baseline prior to placebo and THC administration. But after THC, HFn increased from $34 \pm 3.5$ to $46 \pm 4(p=0.001)$ and $L F / H F$ ratio reduced from $1.53 \pm 0.27$ to 0.95 ( $\mathrm{p}=0.01$ ). Moreover, functional connectivity between the left DLPFC and RVLM increased only after THC administration, and this increase in connectivity was highly positively correlated with the increase in vagal tone (i.e., decreased sympathetic activity), $\mathrm{R}_{2}=0.87, \mathrm{p}<0.000$.

Conclusions: Our results demonstrate that THC administration in the context of chronic pain promotes the functional connectivity between high-order prefrontal modulatory brain areas and the RVLM, the main sympathetic tone regulator. These changes in functional connectivity correlated with increased vagal tone, and were associated with improved CPM and clinically significant analgesia.

Funding: Recanati Funding.

\section{Poster \#54}

Peripheral chemoreflex drives sympathetic activation in the human brainstem and hypothalamus

D.A. Gerlach, J. Manuel, A. Hoff, H. Kronsbein, F. Hoffmann, K. Heusser, A. Diedrich, H. Ehmke, J. Jordan, F. Beissner, J. Tank Institute of Aerospace Medicine, German Aerospace Center (DLR), Cologne, Germany

Introduction: Peripheral carotid chemoreceptors, which raise sympathetic activation at the brainstem level, may serve as targets for 
antihypertensive therapy. However, human peripheral chemoreflex regulation in the brainstem is poorly understood due to lack of suitable methodologies. Therefore, we combined measurements of beatby-beat blood pressure and $\mathrm{SpO}_{2}$, and high-resolution functional magnetic resonance imaging (fMRI) to elucidate human brainstem circuits engaged through hypoxic peripheral chemoreceptor activation.

Methods: We submitted 12 healthy men $(29.7 \pm 6.6$ years; $24.0 \pm 1.86$ $\mathrm{kg} / \mathrm{m}^{2}$ ) to five hypoxic episodes by breathing $10 \%$ oxygen for 180 seconds followed by 90 seconds normoxia during multiband fMRI brain acquisitions. We monitored continuous finger arterial blood pressure using customized hardware, ECG, and $\mathrm{SpO}_{2}$. Brainstem and hypothalamus fMRI images were analyzed to identify nuclei involved in peripheral chemoreflex processing. Systolic blood pressure (SBP) and $\mathrm{SpO}_{2}$ time courses were correlated with the blood-oxygen-level dependent signals with a general linear model.

Results: With hypoxia, $\mathrm{SpO}_{2}$ decreased by $12.32 \pm 3.68 \%(\mathrm{p}<0.01)$, heart rate increased $13.86 \pm 3.47(\mathrm{p}<0.01)$, and SBP decreased with hypoxia $5.45 \pm 5.5 \mathrm{mmHg}(\mathrm{p}<0.01)$. In the brainstem, the nucleus tractus solitarii (t-values: $\mathrm{SpO}_{2}: 5.9$; $\mathrm{SBP}: 4.79$ ), the caudal ventrolateral medulla $\left(\mathrm{SpO}_{2}\right.$ : 5.61; $\left.\mathrm{SBP}: 5.59\right)$, intermediate reticular nucleus $\left(\mathrm{SpO}_{2}\right.$ : 4.7, SBP: 5.98), nucleus ambiguus $\left(\mathrm{SpO}_{2}: 5.03, \mathrm{SBP}\right.$ : 5.59), dorsal motor nucleus of the vagal nerve (SBP: 4.79), and inferior olive $\left(\mathrm{SpO}_{2}\right.$ : 4.7, SBP: 6.16) were identified with high sensitivity and corrected for multiple comparisons $(\mathrm{p}<0.01)$. Furthermore, we observed activation of the following hypothalamic nuclei: paraventricular nucleus $\left(\mathrm{SpO}_{2}: 7.67\right)$, anterior and lateral hypothalamic area $\left(\mathrm{SpO}_{2}: 7.67, \mathrm{SBP}: 4.79\right)$, supraoptic nucleus, and tuberomammillary nucleus $\left(\mathrm{SpO}_{2}: 7.07\right)$.

Conclusion: High-resolution brainstem fMRI during repeated hypoxia traces brainstem circuits engaged by peripheral chemoreceptors. The methodology can be applied to study peripheral chemoreceptor contributions to human cardiovascular disease and may have utility in identifying patients likely to respond to peripheral chemoreceptor modulation.

\section{Poster \#55}

Functional connectivity of the central autonomic network and regulation of parasympathetic tone in health and chronic disease

R. McIntosh ${ }^{1}$, K. Reaves ${ }^{1}$, G. French ${ }^{1}$, J. Lobo ${ }^{1}$, C. Chang ${ }^{2}$ ${ }^{1}$ Department of Psychology, University of Miami, College of Arts and Sciences, Miami, FL, USA; ${ }^{2}$ Computer Science, Electrical Engineering, Computer Engineering, Vanderbuilt University, Nashville, TN, USA

Introduction: With the advancement of functional magnetic resonance imaging (fMRI) analysis there has been growing interest in the physiological changes that may be associated with fluctuations in brain connectivity. Although the field is dominated by findings of functional activation associated with autonomic task performance the task-free approach to resting fMRI allows for the coincidence of brain activation to be linked to changes in HRV without the confounding effect of task manipulation.

Methods: Resting state (rs-fMRI) data from a 7-minute task free trial along with corresponding inter-beat intervals were collected using an MRI-compatible photoplethysmograph (PPG) placed on left index finger. Fifty-four post-menopausal women $(\mathrm{age}=56.13 \mathrm{yrs}, \mathrm{SD}=5.85$, $33 \%$ hypertensive and $46 \% \mathrm{HIV}+$ ) providing artifact free resting fMRI and PPG data were analyzed. Four ROIs - two in the left and right insula and two in the left and right ventromedial prefrontal cortex (VMPFC) - were selected as seed regions for the connectivity analysis based upon their role in parasympathetic regulation. Heart rate variability was calculated as the root mean square deviations (RMSSD) of the heart rate time series. For each subject and seed ROI a whole-brain sliding-window analysis of seed-based functional connectivity was performed in individual space, using 45-s windows with $50 \%$ overlap between adjacent windows, resulting in 15 windows across each rs-fMRI scan.

Results: Covariation of RMSSD with functional connectivity across the resting-state scans was observed between both right and left insula seeds with a cluster of voxels in the right amygdala and the left VMPFC. These activations did not survive peak and cluster-wise correction ( $\mathrm{p}<0.001$, uncorrected). There was also no significant covariation observed between the VMPFC seeds and RMSSD within the whole brain.

Conclusion: These findings suggest functional connectivity of the insula and amygdala may relate to cardiovagal regulation at rest. Further work is needed to elucidate the effect of chronic disease on network function.

Funding: National Heart Lung \& Blood Institute K01 HL139722.

\section{Poster \#56}

HIV-related decrement in respiratory arrhythmia during a deep breathing test

R. McIntosh, K. Reaves, D. Dukenik, J. Lobo, V. Sookdeo Department of Psychology, University of Miami, College of Arts and Sciences, Miami, FL, USA

Introduction: Autonomic function is compromised in chronic human immunodeficiency virus (HIV) infection. Disease-related comorbidities and behavioral factors deplete lung function and alter breath timing and drive in persons living with HIV. These factors may contribute to autonomic dysfunction and impede respiratory arrhythmia in older adult women. This study sought to compare, amongst post-menopausal women, whether change in respiratory sinus arrhythmia (RSA) to a deep breathing varies as a function of HIV-disease status.

Methods: Cardio-autonomic data from 15 post-menopausal women living with HIV (mean=57.27, SD=5.61) and 15 HIV-negative controls (mean=54.73, SD=4.10) were collected during 2 concurrent paced breathing sessions. During the test, subjects laid quietly supine and were asked to breathe at a rate of 12 breaths per minute (BPM) by following along to an audio tape for 2-5 min (until desired respiratory rate was achieved). Subsequently, participants were asked to breath at a rate of 6 BPM. Standard measures of heart rate variability in the time and frequency domain were compared between the two conditions as a function of HIV status. Also, the expiratory-inspiratory ratio (E:I ratio) was calculated based upon the ratio of longest RR interval during expiration and the shortest RR interval during inspiration from 5 sequential cycles.

Results: During the paced breathing test E:I ratio was found to be lower in HIV + women (mean $=1.10, \mathrm{SD}=0.06)$ compared to HIVnegative women (mean=1.16, $\mathrm{SD}=0.08), \mathrm{F}(1,28)=5.11, \mathrm{p}=0.032$. Repeated measures analysis of variance (ANOVA) largely revealed no differences in HRV parameters between the two breathing conditions, i.e., RMSSD, SDNN, LF, HF, LF:HF. There was, however, a trend level effect for BPM rate on the reduction is RSA between the two conditions $\mathrm{F}(1,28)=3.60, \mathrm{p}=0.068$ ).

Conclusion: Postmenopausal women living with HIV show lower respiratory arrhythmia during paced breathing.

Funding: National Heart Lung \& Blood Institute, K01 HL139722. 


\section{Poster \#57}

\section{Normal values of quantitative thermoregulatory sweat testing (Q-TST)}

\author{
A. Arvantaj, J. Robinson, C. Geiger, B. Katirji \\ Neurological Institute, University Hospitals Cleveland Medical \\ Center, Cleveland, OH, USA
}

Introduction: Thermoregulatory sweat testing (TST) evaluates the distribution of sweating by a change in color of an indicator powder (Alizarin Red). It is a technically challenging test and may result in false readings. TST can pick up the disorders when they are in advanced stages and have reached to a level that cannot cause a color change in Alizarin powder. We contemplate that instead of binominal variable of "sweat absent/sweat present" we can measure sweat output with a continuous variable (microliter/ $\mathrm{cm}^{2}$ ). Using a continuous variable may be helpful to diagnose the central thermoregulatory disorders (e.g., multiple system atrophy) earlier in their course. The first step towards this goal is to find the normal values in healthy individuals.

Objective: To quantify the volume of sweating on extremities during 30 minutes of extreme heat.

Methods: TST was done at 50C heat and 50\% humidity and multiple photos was taken at the end of 30-minute cycle. A Q-Sweat device was used to collect sweat from foot, proximal leg, dorsal hand and forearm with $0.79 \mathrm{~cm}^{2}$ capsules. Digital photographic pictures were reviewed by two reviewers who were blind to clinical situation and quantitative sweat collection. Only patients with normal TST (complete color change at all four recording sites) by both reviewers were included. Results: 23 patients (10 females) were included. Mean (and standard deviation) of sweat outputs (microliter $/ \mathrm{cm}^{2}$ ) in Male cases who reported as having a normal TST by both reviewers were: foot 4.6 (1.7), calf 8.4 (3.2), hand 15.2 (3.4) and forearm 8.1(3.5). And in females: foot 4.3 (2.1), calf 6.7 (4.2), hand 11.3 (4.2) and forearm 8.1 (2.5).

Conclusion: In this study we retrospectively reported normal volume of sweat output in response to 30 minutes of extreme (but controlled) heat with a continuous variable (microliter/ $\mathrm{cm}^{2}$ ). Simultaneous collection of sweat output by Q-Sweat device during TST may reduce misinterpretation of TST and help with early diagnosis of central thermal dysregulations. A prospective study in healthy volunteers is needed for confirmation.

\section{Poster \#58}

\section{Experience from a multidiscliplinary care clinic for multiple system atrophy}

S. Vernino, E. Golden, E. Kent, G. Huet, M. Bryarly

Department of Neurology \& Neurotherapeutics, UT Southwestern Medical Center, Dallas, TX, USA

Objective: To report the 4-year experience from the UT Southwestern multidiscliplinary care clinic for multiple system atrophy patients (MSA).

Background: MSA is a progressive neurodegenerative disorder with prominent autonomic failure. While there are some symptomatic therapies, there is no disease-modifying treatment for MSA. Patients and their caregivers experience a large impact on quality of life.

Methods: Since September 2015, UT Southwestern has offered a multidisciplinary care clinic for MSA patients. All patients attending the clinic are invited to participate as research subjects and attend the clinic 3 times a year (every 4 months). At each visit, clinical assessments (including UMSARS) and patient questionnaires (including COMPASS-31, quality of life and caregiver burden) are administered. The core care team consists of neurology, physiatry, palliative care, social work and nutrition. Additional components of the multidisciplinary team include urology, otolaryngology, sleep, speech therapy and a monthly patient/caregiver support conference. Results: Since initiation, 88 MSA patients consented to be part of the MSA clinic project; 61 patients have had more than one visit to the clinic with an average duration of follow-up of 16 months. Over time, clinical status worsened as expected with progression of UMSARS scores by about 3.8 points per year (Part 1) or 5 points per year (Part 2 ). However, measures of caregiver burden and quality of life progressed minimally over time, suggesting a benefit of care model. Participants indicated that the multidisciplinary care approach provided value in terms of integration of care, education and improved psychosocial support.

Conclusions: A multidisciplinary care model is particularly valuable in MSA. Our model could be applied more broadly at other institutions. The longitudinal collection of data can assist in defining the natural history of the disease and impact of integrated care model on quality of life.

Funding: Rex Griswold Foundation.

\section{Poster \#59}

\section{Neurogenic orthostatic hypotension in patients referred for evaluation in a Chilean autonomic clinic}

\section{J. Idiaquez, O. Trujillo, J.F. Idiaquez, R. Iturriaga}

Departments of Neurology and Physiology, Pontificia Universidad Catolica de Chile, Santiago, Chile; Department of Neurology, Universidad del Desarrollo, Santiago, Chile

Aim: The aim of this retrospective study was to describe autonomic symptoms and severity of sympathetic and cardiovagal dysfunction in patients with neurogenic orthostatic hypotension associated with synucleinopathies.

Patients and Methods: 50 patients, grouped in: Parkinson disease (PD) (14), multiple system atrophy cerebellar (MSA-C) (10), multiple system atrophy Parkinson (MSA-P) (19), and pure autonomic failure phenotype (PAF-P) (7). Autonomic symptoms domains were evaluated with the SCOPA-autonomic scale. Autonomic tests: systolic blood pressure (BP) and heart rate (HR) change on standing, cardiovagal: HR change on deep breathing (HRDB), sympathetic skin responses (SSR) in palm and sole. Data: Mean \pm SEM, one-way Anova followed by Dunnet multiple comparisons.

Results: Symptoms: Total SCOPA score was not different among groups (25.9 to 33.3) but in the PAF-F group the urinary and gastrointestinal scores were lower compared to the PD and MSA-C and MSA-P groups. Autonomic function tests: systolic BP fall on standing was higher in PAF-F $(73.9 \pm 22.6 \mathrm{mmHg})$ related the other groups $\mathrm{PD}$ $(31.2 \pm 12.8 \mathrm{mmHg})$, MSA-C $(47.8 \pm 22.7 \mathrm{mmHg})$ and MSA-P $(53.2 \pm 22.1 \mathrm{mmHg})$. HR increase on standing was not different PAFF ( $12.7 \pm 5.3$ beat $/ \mathrm{min})$, PD $(10.1 \pm 14.3$ beat $/ \mathrm{min})$, MSA-C $(9.4 \pm 17.3$ beat $/ \mathrm{min})$ and MSA-P $(9.3 \pm 9.9$ beat $/ \mathrm{min})$. Abnormal cardiovagal and sudomotor tests were, respectively, PAF-F $(6 / 7,6 / 7)$, PD $(7 / 14,1 / 14)$ MSA-C (10/10, 3/10) and MSA-P (18/20, 15/20).

Conclusions: PAF-F group showed lower urinary and gastrointestinal symptoms scores. Sympathetic and cardiovagal tests showed mayor dysfunction in PAF-F group. MSA-P and MSA-C showed greater cardiovagal dysfunction than in PD group. MSA-P showed greater sudomotor dysfunction than in MSA-C and PD groups 


\section{Poster \#60}

Cutaneous alpha-synuclein deposition in idiopathic REM sleep behavioral disorder

M.G. Miglis ${ }^{1}$, J. Zitser ${ }^{2}$, S. Rajan ${ }^{3}$, E. During ${ }^{1}$, R. Freeman ${ }^{3}$, C.H. Gibbons ${ }^{3}$

${ }^{1}$ Department of Neurology, Stanford University, Stanford, CA, USA; ${ }^{2}$ Global Brain Health Institute, Department of Neurology \&

Neurological Sciences, University of California, San Francisco, San Francisco, CA, USA; ${ }^{3}$ Department of Neurology, Beth Israel Deaconess Medical Center, Harvard Medical School, Boston, MA, USA

Background: Phosphorylated $\alpha$-synuclein (P-SYN) has been detected within cutaneous autonomic nerve fibers of patients with central nervous system (CNS) $\alpha$-synucleinopathies such as Parkinson's disease and multiple system atrophy, and more recently in patients with idiopathic REM sleep behavior disorder (iRBD). We now report our data on the deposition of P-SYN in patients with iRBD.

Objective: To define the deposition of P-SYN in individuals with iRBD.

Methods: Twenty-one individuals had quantified neurological and cognitive examinations, autonomic function testing, sleep studies, smell testing, questionnaires and punch skin biopsies taken from multiple proximal and distal sites. Two skin biopsies were stained for PGP9.5 and P-SYN and results compared between those with and without $\alpha$-synuclein.

Results: P-SYN was detected in 10/21 (48\%) of iRBD patients. The groups were of similar age, gender, and duration of RBD. The groups had similar MOCA, UMSARS, CASS and quality of life scores. Patients positive for P-SYN had lower UPSIT scores (21.2 \pm 7.5 vs. $32.8 \pm 6.7, \mathrm{P}<0.01)$, lower QSART values at multiple sites $(\mathrm{P}<0.05)$ and lower intra-epidermal nerve fiber density at the distal leg. The P-SYN positive group had a greater fall in systolic blood pressure on head-up tilt $(\mathrm{P}<0.05)$ and greater sympathetic adrenergic impairment in autonomic testing (CASS score $\mathrm{P}<0.05$ ). There were trends towards higher SCOPA-AUT, COMPASS-31 and total CASS scores $(\mathrm{P}<0.1)$ in the $\mathrm{P}-\mathrm{SYN}$ group.

Discussion: In patients with iRBD, the presence of P-SYN was seen in approximately half of cases prior to any other CNS manifestations of neurodegenerative disease. Those positive for P-SYN had several features suggestive but not diagnostic of a more generalized synucleinopathy. Longitudinal follow-up is necessary to determine the long-term implications of cutaneous P-SYN.

Funding: Study supported by NIH U54-NS065736.

\section{Poster \#61}

\section{Sudomotor abnormalities in idiopathic REM sleep behavior disorder}

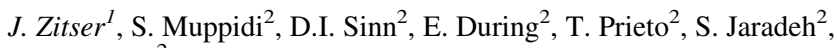
M.G. Miglis ${ }^{2}$

${ }^{1}$ Global Brain Health Institute, Department of Neurology \& Neurological Sciences, University of California, San Francisco, San Francisco, CA, USA; ${ }^{2}$ Department of Neurology \& Neurological Sciences, Stanford University, Stanford, CA, USA

Background: Several publications have demonstrated post-ganglionic sudomotor impairment and small fiber neuropathy (SFN) in patients with Parkinson's disease (PD), suggesting that epidermal denervation may be an early feature of PD. More recently, idiopathic REM sleep behavior disorder (iRBD) has also been associated with sudomotor abnormalities, however, data are limited. We now report on the prevalence of post-ganglionic sudomotor impairment in a cohort of patients with iRBD.

Objective: To evaluate the prevalence of sudomotor impairment in a cohort of patients with iRBD compared to patients with PD.

Methods: We performed a retrospective chart review of all patients seen in the Stanford autonomic clinic from 2013-2019 who underwent sudomotor testing with the quantitative sudomotor axon reflex test (QSART). We identified three groups for analysis: 1) iRBD, 2) PD with RBD, and 3) PD without RBD. Patients with iRBD were diagnosed by a sleep specialist and all patients had video polysomnography confirmation of this diagnosis. Exclusion criteria included a diagnosis of peripheral neuropathy, diabetes mellitus, alcoholism, chemotherapy, or other systemic disorder associated with SFN.

Results: iRBD patients $(\mathrm{n}=20)$ were of similar age and gender to PD with RBD ( $n=21)$ and PD without RBD ( $n=17)$. The PD with RBD group demonstrated the most sudomotor abnormalities (sudomotor CASS $1.285 \pm 1.23)$, followed by iRBD $(0.55 \pm 0.944)$ and PD without RBD (0.294 $\pm 0.58, \mathrm{p}=0.007)$. Most patients demonstrated a nonlength dependent pattern of sweat loss.

Discussion: QSART abnormalities are common in patients with iRBD, and intermediate between those of PD with RBD and PD without RBD. These findings support the concept that cutaneous neuropathy occurs early in the course of the alpha-synucleinopathies, and may reflect a peripheral deposition of alpha-synuclein that occurs in tandem with deposition in the central nervous system.

\section{Poster \#62}

Anti-cholinergic receptor antibodies, cardiovascular hemodynamics and dysautonomia symptoms relationship in pure autonomic failure: a two-year follow-up

F. Barbic ${ }^{1,5}$, M. Minonzio ${ }^{1}$, B. Cairo ${ }^{2}$, D. Shiffer ${ }^{1}$, E. Brunetta $^{1}$, F.

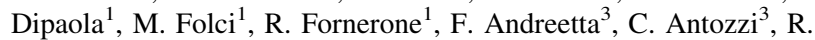
Mantegazza $^{3}$, A. Porta ${ }^{2,4}, R$. Furlan ${ }^{1,5 *}$

${ }^{1}$ Department of Internal Medicine, Humanitas Research Hospital, Rozzano, Milan, Italy; ${ }^{2}$ Department of Biomedical Sciences for Health, University of Milan, Milan, Italy; ${ }^{3}$ Neuroimmunology and Neuromuscular Diseases Unit, I.R.C.C.S. Istituto Neurologico 'C. Besta', Milan, Italy; ${ }^{4}$ Department of Cardiothoracic, Vascular Anesthesia and Intensive Care, IRCCS Policlinico San Donato, San Donato Milanese, Milan, Italy; ${ }^{5}$ Department of Biomedical Sciences, Humanitas University, Rozzano, Italy

Background: Plasma anti-alpha3 and alpha7 ganglionic cholinergic receptor (Anti-AChR) antibodies (Abs) may be present in patients initially diagnosed with pure autonomic failure (PAF), accounting for an autoimmune mechanism of the dysautonomia (autoimmune autonomic ganglionopathy, AAG).

Aims: To evaluate the time course of Abs plasma titer, hemodynamics and symptoms intensity and whether Abs negative PAF patients might develop Anti-AChR Abs over time.

Methods. We enrolled (T0) 23 patients who fulfilled the diagnostic criteria for PAF (age $68 \pm 2$ years, $12 \mathrm{~F}$ ). All patients underwent antiAChR Abs plasma levels, heart rate (HR), arterial pressure (AP) and plasma norepinephrine (NE) evaluation while supine and during $75^{\circ}$ head-up tilt. Dysautonomia symptoms were assessed by the COMPASS 31 score. The same parameters were re-evaluated at $12(\mathrm{~T} 1, \mathrm{n}=23)$ and 24 months $(\mathrm{T} 2, \mathrm{n}=15)$. Values are expressed as mean $\pm \mathrm{SE}$.

Results: At T0, 7 patients were anti-AChR Abs positive (30\%). At T0, rest: HR $69 \pm 2 \mathrm{~b} / \mathrm{min}$; AP $154 \pm 7 / 81 \pm 3 \mathrm{mmHg}$, NE $128 \pm 14 \mathrm{pg} / \mathrm{ml}$. During tilt: HR $68 \pm 2 \mathrm{~b} / \mathrm{min}, \mathrm{AP} 81 \pm 5 / 45 \pm 3 \mathrm{mmHg}, \mathrm{NE} 180 \pm 20 \mathrm{pg} /$ 
ml. At T1, rest: HR 68 \pm 2 b/min; AP $154 \pm 6 / 82 \pm 4$ mmHg, NE $132 \pm 14$ $\mathrm{pg} / \mathrm{ml}$. During tilt: HR $70 \pm 2 \mathrm{~b} / \mathrm{min}$; AP $78 \pm 5 / 42 \pm 3 \mathrm{mmHg}, \mathrm{NE}$ $146 \pm 17 \mathrm{pg} / \mathrm{ml}$. At T2, rest: HR $68 \pm 3 \mathrm{~b} / \mathrm{min}$; AP $151 \pm 9 / 88 \pm 4 \mathrm{mmHg}$, NE $124 \pm 33 \mathrm{pg} / \mathrm{ml}$. During tilt: HR $71 \pm 3 \mathrm{~b} / \mathrm{min}$; AP $97 \pm 8 / 54 \pm 5$ $\mathrm{mmHg}, \mathrm{NE} 174 \pm 43 \mathrm{pg} / \mathrm{ml}$. Symptoms scores: At T0: orthostatic intolerance $(6.8 \pm 0.5)$, secretion disorders $(3.0 \pm 0.4)$, gastrointestinal tract disorders $(10.5 \pm 1.1)$, visual disorders $(7.0 \pm 0.8)$. At T1: orthostatic intolerance $(6.3 \pm 0.7)$, secretion disorders (3.2 \pm 0.5$)$, gastrointestinal tract disorders $(9.4 \pm 2.0)$, visual disorders (5.9 \pm 0.7$)$. At T2: orthostatic intolerance (5.6 \pm 1.0$)$, secretion disorders $(2.9 \pm 0.5)$, gastrointestinal tract disorders (9.1 \pm 0.9$)$, visual disorders $(5.9 \pm 0.7)$. All above indices remained unchanged during $\mathrm{T} 1$ and $\mathrm{T} 2$ and no novel Anti-AChR Abs positivity was observed in Abs negative patients.

Conclusions. There is no evidence of disease progression nor of new Abs appearance in this population.

Funding: Supported by the Italian Ministry of Health, grant \# RF2013-02355242.

\section{Poster \#63}

\section{A phase-2 randomized placebo-controlled trial of sirolimus in multiple system atrophy: protocol, recruitment and preliminary adverse event profile}

J.-A. Palma, J. Martinez, S. Simon, L. Norcliffe-Kaufmann, H. Kaufmann

Department of Neurology, Dysautonomia Center, New York University School of Medicine, New York, NY, USA

Objective: We present the protocol, recruitment numbers, and preliminary adverse event profile of patients enrolled in a single-center phase-2 futility trial using sirolimus for multiple system atrophy (MSA) (ClinicalTrials.gov: NCT03589976).

Background: In patients with MSA, autophagy is impaired and misfolded aSyn accumulates in neurons and glia, causing neurodegeneration. Sirolimus, a medication that has been approved by the U.S. Food and Drug Administration for chronic treatment in humans for a variety of disorders for almost 20 years, is a potent activator of autophagy. We hypothesize that treatment with sirolimus might activate autophagy of $\alpha$ Syn resulting in reduced neurodegeneration and slower progression of the neurological deficits in patients with MSA.

Methods: Single-center, randomized, placebo-controlled, phase-2 futility clinical trial to determine if sirolimus is of sufficient promise to slow the disease progression of patients with MSA, prior to embarking on a large-scale and costly phase- 3 study to assess its efficacy. Non-futility will offer strong support for a phase-3 trial to detect clinical efficacy. We will enroll 56 patients with a 3:1 (sirolimus:placebo) randomization. We expect to complete enrollment in 2 years.

Results: The first patient was screened and enrolled in September 2018. By May 2019, 35 patients had been screened and 31 had been enrolled and randomized. By October 2019 we expect to have enrolled 43 patients ( $76 \%$ of our final target enrollment). Common adverse events included oral ulcers, abdominal discomfort and diarrhea/loose stools. Recruitment and adverse events will be updated by the time of this abstract presentation.

Conclusions: This is the first time sirolimus or analogs are being used clinically with the aim of slowing disease progression in patients with neurodegenerative disorders. Our observations may offer strong support for a phase-3 trial to confirm the efficacy of sirolimus in MSA.

Funding: NIH (R01NS107596-01).

\section{Poster \#64}

Impact of spinal cord injury on maximal cutaneous vasodilation, myogenic and venoarteriolar responses

M. Trbovich, Y. Wu, W. Koek, J. Zhao, A. McMahon, D. Kellogg Department of Medicine, University of Texas Health Science Center at San Antonio, San Antonio, TX, USA

Background: Vascular dysfunction after spinal cord injury (SCI) results in orthostatic hypotension $(\mathrm{OH})$ and contributes to morbidity. Whether the peripheral blood pressure regulating venoarteriolar (VAR) and myogenic (MYO) responses are centrally controlled is unclear. Persons with SCI have varying degrees of intact central sympathetic nervous system (SNS) control proportional to their level of injury; and thus serve as an experiment of nature to investigate if VAR and MYO are centrally controlled. Furthermore, endothelial function and structural changes of the microvasculature post-SCI, which predict cardiovascular risk, are under-explored and differences between those with tetraplegia (TP) and paraplegia (PP) never reported.

Methods: Eight able-bodied (AB), 7 TP, and 8 PP persons participated. One forearm and calf were treated topically with lidocaine $2.5 \%$ prilocaine $2.5 \%$ while contralateral limb served as a control. Laser doppler flowmetry (LDF) measured changes in vascular resistance (VR) during limb dependency and cuff inflation. All sites were then heated to skin temperature of (Tloc) $34^{\circ} \mathrm{C}$, then up to $42^{\circ} \mathrm{C}$ for maximal vasodilation and cutaneous vascular conductance (CVC) measured.

Results: There was no significant difference in change in VR among groups (TP, PP vs. AB) or limbs (arm vs. leg) during cuff inflation or limb dependency; or in CVC at Tloc $34^{\circ} \mathrm{C}$ between groups or limb. There was a significant difference in CVC at Tloc $42^{\circ}$ in the lower limb of persons with TP $(p=0.011)$ and $P P(p=0.33)$ when compared to $\mathrm{AB}$ persons.

Conclusion: VAR and MYO remain intact in SCI persons, however maximal CVC in the lower limbs of SCI is higher than AB persons. This may be due to altered structural adaptations to the resistance vessels after SCI.

Funding: NIH-National Institute of Neurological Disorders and Stroke R03NS083961.

\section{Poster \#65}

Acute effects of mechanical somatosensory stimulation on cardiac autonomic control and its relationship with postural control in patients with Parkinson's disease

B.M. Franco ${ }^{1}$, N.R. Marques ${ }^{1}$, V.C. Moreno ${ }^{2}$, M.H. Kuroda ${ }^{2}$, F.A. Barbieri ${ }^{2}$, A.R. Zamunér $^{3}$

${ }^{1}$ Center of Health Sciences, Universidade do Sagrado Coração, Bauru, São Paulo, Brazil; ${ }^{2}$ MOVI-LAB Human Movement Research Lab, Physical Education Department, Post-Graduation Program in Movement Sciences, UNESP São Paulo State University, Bauru, Brazil; ${ }^{3}$ Department of Kinesiology, Universidad Católica del Maule, Talca, Maule, Chile

Background: Parkinson's disease (PD) is a neurodegenerative pathology characterized predominantly by motor symptoms, such as rest tremor, stiffness and compromised postural control. Besides motor abnormalities, cardiovascular autonomic dysfunction is also common in this population. Automatic mechanical peripheral stimulation (AMPS) has been proposed as a new therapy to improve gait and dysautonomia in PD patients. However, whether the improvement 
in postural control is related to the improvement of dysautonomia is unknown. Objective: To evaluate the acute effect of AMPS on cardiac autonomic control and postural control and their association in patients with PD.

Methods: Eighteen volunteers with PD participated in the study. Participants were randomly assigned to two intervention sessions: 1) "real" AMPS protocol; 2) placebo. A two-week washout period was considered between sessions. Outcomes were assessed before and after each session. Postural control was analyzed using a triaxial accelerometer, quantifying the displacement of the center of pressure (COP) in anteroposterior and mid-lateral direction. RR intervals were recorded in supine and standing postures using a heart rate monitor (Polar V800). Heart rate variability (HRV) analysis was performed using time domain (SDNN and RMSSD) and spectral analysis (low frequency, LF and high frequency, HF components). Wilcoxon and Spearman tests were applied $(\alpha=5 \%)$.

Results: One AMPS session significantly increased RMSSD ( $\mathrm{p}=0.01)$, $\mathrm{LF}(\mathrm{p}=0.02)$ and HF $(\mathrm{p}=0.03)$ in the supine posture. Correlation analysis revealed significant relationship between $\Delta \mathrm{COP}$ (post minus baseline) and $\triangle \mathrm{HRV}$ indices (standing minus supine) post intervention (SDNN: Rs=0.85, $\mathrm{p}<0.0001$; RMSSD: Rs $=0.55, \mathrm{p}=0.03$; LF: $\mathrm{Rs}=0.55, \mathrm{p}=0.02$; and HF: $\mathrm{Rs}=0.64, \mathrm{p}<0.01$ ).

Conclusion: One single session of AMPS acutely improved the cardiac autonomic control in patients with PD. Furthermore, the greater the improvement in cardiac autonomic control, the greater the improvement on postural control. Thus, dysautonomia may play a role in postural control abnormalities.

Funding: CONICYT-FONDECYT de Iniciación: 11180310.

\section{Poster \#66}

Multiple functional abnormalities in cardiac sympathetic nerves revealed by a comprehensive computational model in Lewy body diseases

\section{D.S. Goldstein ${ }^{1}$, M. Pekker ${ }^{2}$, G. Eisenhofer ${ }^{3}$, Y. Sharabi ${ }^{4}$}

${ }^{1}$ Autonomic Medicine Section (formerly Clinical Neurocardiology Section), CNP/DIR/NINDS/NIH, Bethesda, MD, USA; ${ }^{2}$ University of Alabama at Huntsville, Huntsville, AL, USA; ${ }^{3}$ University of Dresden, Dresden, Germany; ${ }^{4}$ Tel Aviv University, Tel Aviv, Israel

Background: Lewy body diseases, a family of aging-related neurodegenerative disorders, entail loss of the catecholamine dopamine in the nigrostriatal system and equally severe deficiency of the closely related catecholamine norepinephrine in the heart. The myocardial noradrenergic lesion is associated with major non-motor symptoms and decreased survival. Numerous mechanisms determine norepinephrine stores, and which of these are altered in Lewy body diseases has not been examined in an integrated way. We used a computational modeling approach to assess comprehensively pathways of cardiac norepinephrine synthesis, storage, release, reuptake, and metabolism in Lewy body diseases. Application of a novel kinetic model identified a pattern of dysfunctional steps contributing to norepinephrine deficiency. We then tested predictions from the model in a new cohort of Parkinson disease patients.

Methods: Rate constants were calculated for 17 reactions determining intra-neuronal norepinephrine stores. Model predictions were tested by measuring post-mortem apical ventricular concentrations and concentration ratios of catechols in controls and patients with Parkinson disease.

Results: The model identified low rate constants for three types of processes in the Lewy body group-catecholamine biosynthesis via tyrosine hydroxylase and L-aromatic-amino-acid decarboxylase, vesicular storage of dopamine and norepinephrine, and neuronal norepinephrine reuptake via the cell membrane norepinephrine transporter. Post-mortem catechols and catechol ratios confirmed this triad of model-predicted functional abnormalities.

Conclusions: Denervation-independent impairments of neurotransmitter biosynthesis, vesicular sequestration, and norepinephrine recycling contribute to the myocardial norepinephrine deficiency attending Lewy body diseases. A proportion of cardiac sympathetic nerves are "sick but not dead," suggesting targeted disease-modification strategies might retard clinical progression.

Funding: Division of Intramural Research, NINDS, NIH.

\section{Poster \#67}

The relationship between sympathetic modulation and central blood pressure in African American and Caucasian women

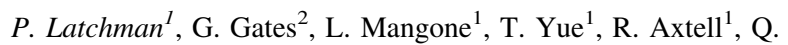

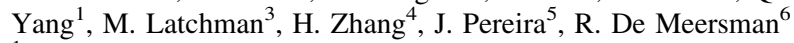
${ }^{1}$ Exercise Science Department, Southern Connecticut State University, New Haven, CT, USA; ${ }^{2}$ Pediatrics Department, Albert Einstein College of Medicine, Bronx, NY, USA; ${ }^{3}$ G.C. Foster College of Physical Education and Sports, Spanish Town, Jamaica; ${ }^{4}$ Hunan First Normal University, Changsha, China; ${ }^{5}$ Yale New Haven Hospital, New Haven, CT, USA ${ }^{6}$ American University of Antigua, Antigua

Introduction: African American women (AAW) are at greater risk for cardiovascular disease (CVD) vs. Caucasian women (CW). There is an association between sympathetic modulation (SM) and peripheral blood pressure (PBP). AAW have demonstrated higher values of SM and PBP vs. CW. Central blood pressure (CBP) and other aortic pressures, example: augmentation pressure (AP) and augmentation index (AIx), are more indicative of negative cardiovascular events vs. PBP. While much is known about the association between SM and $\mathrm{PBP}$, there is a paucity of research examining the association between SM and CBP based on ethnicity.

Aim: To determine the association between SM, CBP, and other aortic pressures in normotensive AAW and CW.

Methods: Thirty-four (AAW-17; CW-17) healthy, age, height, and weight-matched women were examined for SM, central systolic blood pressure (CSBP), central diastolic blood pressure (CDBP), AP and AIx, at a heart rate of 75-beats.min-1 (AIx@75); SM being the ratio of the low frequency (LF) to high frequency (HF) - (LF/HF) power of heart rate variability via power spectral density analysis, while CSBP, CDBP, AP and AIx@75 were determined via applanation tonometry. Results: Pearson correlation indicated that the LF/HF ratio was positively associated with CSBP, $\mathrm{r}=0.65(\mathrm{P}<0.01)$ and $\mathrm{CDBP}, \mathrm{r}=0.56$ $(\mathrm{P}=0.01)$ in $\mathrm{AAW}$ but not in $\mathrm{CW}$; CSBP, $\mathrm{r}=-0.04,(\mathrm{P}=0.85)$ and CDBP, $r=0.19(\mathrm{P}=0.44)$. SM was not associated with AP and AIx@ 75 in women.

Discussion: There are differences in the association between SM and CBP in AAW versus CW. It appears that increased SM to the heart of AAW have more deleterious impacts on cardiovascular health in AAW versus CW.

Conclusion: Preliminary data suggest that SM is significantly associated with CBP in AAW but not CW. SM is not associated with other aortic pressures in women. 


\section{Poster \#68}

\section{Autonomic dysfunction and its association with painful and painless neuropathy}

\section{R. Lüddecke, R. Baron, J. Gierthmühlen}

Division of Neurological Pain Research and Therapy, Department of Neurology, University Hospital Kiel, Germany

Background and Aims: Autonomic dysfunction can occur in polyneuropathy when small nerve fibers are involved. Both pain and autonomic nervous system functions are peripherally mediated by small nerve fibers. Autonomic neuropathy can affect the vagus nerve and its pain inhibitory capacity, thus increasing sensitivity to pain. This study aimed to describe the occurrence of autonomic dysfunction in painful and painless neuropathies and evaluate the relationships between autonomic function and the perception of pain.

Methods: So far, twenty-one patients with painful polyneuropathy (age $66.1 \pm 11.9$ years) and 10 patients with painless neuropathy $(64.7 \pm 13.1$ years) were enrolled (end of recruitment planned for August 2019). Heart rate variability at rest, deep-breathing and Ewing test, tilt-table testing, cutaneous vasoconstrictor (CVC) test as well as quantitative sensory testing (QST) and conditioned pain modulation (CPM) response were examined.

Results: In the group of patients enrolled so far, there was a trend towards a higher frequency of orthostatic hypotension (15\% with painful vs. $0 \%$ with painless neuropathy, p n.s.) and decreased heart rate variability $(27.8 \%$ with painful compared to $0 \%$ with painless neuropathy, p n.s.) in the painful compared to painless neuropathy. CVC revealed similar results in both groups. CPM response did not differ between painful and painless neuropathy, however, the conditioning stimulus was felt less painful after one minute in painful compared to painless neuropathy $(\mathrm{p}<0.05)$.

Conclusion: Results suggest differences in presence of pain and autonomic dysfunction in painful versus painless neuropathy. Final analysis has to show whether presence of pain and autonomic dysfunction depends on severity of neuropathy including small-fiber involvement and whether diminished vagal function due to neuropathy is associated with pain disinhibition.

Funding: This work was supported by the European Union's Horizon 2020 research and innovation programme under grant agreement No 633491.

\section{Poster \#69}

Dysautonomia in Guillain-Barré syndrome: an under-recognized and potentially life-threatening phenomenon

\section{A.V. Varma-Doyle, H. Gould \\ Department of Neurology, Louisiana State University Health Sciences Center, New Orleans, LA, USA}

Introduction: Dysautonomia can occur as the initial presentation of Guillain-Barré syndrome (GBS) and can be an even greater contributor to morbidity and mortality than respiratory dysfunction. GBS is an acute autoimmune demyelinating polyneuropathy that typically presents with ascending weakness and areflexia after a gastrointestinal/respiratory or viral infection. Autonomic dysfunction in GBS is unrelated to severity of peripheral nervous system involvement and is therefore under-recognized. This fact coupled with the variability in symptom onset, often leads to mismanagement of dysautonomia in GBS
Case Report: A 37-year-old-woman with a history of diabetes mellitus and end stage renal disease presented with ascending weakness in addition to feeling dizzy and light-headed, after recent recovery from a gastrointestinal infection. On general exam, she was mildly tachycardic. Neurologically, she demonstrated motor weakness with areflexia and proprioceptive \& vibratory deficits bilaterally in the lower extremities. The results of CSF analysis revealing a high cell count/low protein supported our suspicion of GBS. Her respiratory function was normal, and she was managed in a non-ICU setting. Initiation of IVIG therapy resulted in signs of improvement, however, during dialysis, the patient experienced a bradycardic episode followed by irreversible cardiac arrest.

Conclusion: Autonomic dysfunction is a subtle, potentially lifethreatening and treatable feature of GBS. We report a case of GBSdysautonomia that presented with early features of light-headedness and borderline tachycardia that illustrate the necessity of screening for and management of dysautonomia in GBS. ICU level of care with close cardiac monitoring is advised for patients with GBS even with stable respiratory function in the setting of comorbid conditions like diabetes mellitus and end stage renal disease as these conditions are also associated with dysautonomia, and can exacerbate it. Early identification and attention to managing autonomic involvement can significantly reduce the risk of morbidity and mortality in the disease.

\section{Poster \#70}

Hereditary sensory and autonomic neuropathy type IV: casereport and review of immune dysfunction in the disease

A.V. Varma-Doyle, M. Marble, L. McBride, A. Tilton

Department of Neurology, Louisiana State University Health Sciences Center, New Orleans, LA, USA

Introduction: Hereditary sensory and autonomic neuropathy type IV (HSAN IV) is an autosomal recessive disorder due to mutations of the neurotrophic tyrosine receptor kinase-1 (NTRK1) gene. The NTRK1 receptor is present on cells of the autonomic and the immune system and has affinity for nerve growth factor which when bound activates a cascade of functions important for cell growth, differentiation and survival; in patients with HSAN IV there is a dysfunction in this pathway. The disease presents in infancy, and manifests as sensory dysfunction from lack of unmyelinated fibers and autonomic dysfunction from lack of sweat gland innervation. Mental retardation, self-mutilating injuries from pain insensitivity and hyperpyrexia from defects in sweating are cardinal features of the disorder.

Case Report: We report a 2-year-old girl who initially presented with periodic fevers and self-mutilation of her hands, tongue, and lips. Genetic testing revealed homozygosity for the frameshift mutation c.543delG, confirming a diagnosis of HSAN IV. She later presented with insidious onset of upper extremity paralysis after recovery from a respiratory tract infection. Laboratory results were evident for leukopenia and thrombocytopenia during varying time periods of presentation. MRI cervical spine revealed a mass compressing C3-C7 which when debulked was evident for chronic inflammatory cells on histopathological examination. The etiology of the mass showed no link to prior trauma or infection. The development of an inflammatory mass post viral infection, rapid decline of cell counts with resolution post steroid administration suggested possible autoimmune phenomena in her case.

Conclusion: Innate immunity involving neutrophil recruitment and macrophage function is shown to be disrupted due to defective NGFTRKA signaling in HSAN IV. Adaptive immunity deficits from B and $\mathrm{T}$ cell deficits are also evident in HSAN IV patients. We present a case-report, and review immune dysfunction in HSAN IV, with 
recommendations for screening and surveillance of immunological dysfunction in the disease.

\section{Poster \#71}

Familial dysautonomia and congenital adrenal hyperplasia: one patient with two rare genetic disorders

B.J. Balgobin, J.-A. Palma, L. Norcliffe-Kaufmann, H. Kaufmann Department of Neurology, Dysautonomia Center, New York University School of Medicine, New York, NY, USA

Objective: To describe an unusual case of a young girl of Jewish Ashkenazi descent with two rare genetic disorders: familial dysautonomia and congenital adrenal hyperplasia.

Methods: Case report.

Results: Female patient presenting with hypotonia, failure to thrive, recurrent vomiting and frequent lower respiratory tract infections during the first year of life. Her physical exam disclosed genital ambiguity and, because an older sister had a diagnosis of congenital adrenal hyperplasia, the diagnosis was suspected in this case, and confirmed by low levels of cortisol and aldosterone. Targeted genetic testing showed homozygosis for a pathogenic variant in the CYP21A2 gene (c.293-13C $>$ G, aka I2G, well-described in Jewish Ashkenazi patients) and heterozygosis for 7 other variants in the same gene. She was started on fludrocortisone for mineralocorticoid replacement therapy. Additional signs and symptoms were identified including frequent aspiration pneumonias prompting a gastrostomy tube placement at 6 months of age, emotionally-induced episodes of face, hand and feet blotching, frequent falls, impaired sensitivity to pain, and lack of tears. Additional genetic testing at age 2 disclosed homozygous copies of the founder mutation variant of familial dysautonomia (variant IVS20+6 T $>$ C) in the IKBKAP gene.

Conclusion: The presence of one rare genetic disorder does not preclude the presence of another rare genetic disorder. Signs and symptoms not consistent with one diagnosed genetic disorder should prompt suspicion of additional causes.

Funding: Familial Dysautonomia Foundation.

\section{Poster \#72}

Methionine sulfoxide reductase deficiency exacerbates metabolic dysfunction in mice fed a high-fat diet: implications in metabolic syndrome

\section{R. Sabharwal ${ }^{1}$, L. Yang ${ }^{1}$, M.W. Chapleau ${ }^{1,2,3}$}

Departments of ${ }^{1}$ Internal Medicine and ${ }^{2}$ Molecular Physiology \& Biophysics, University of Iowa, Iowa City, IA, USA; ${ }^{3}$ Veterans Affairs Medical Center, Iowa City, IA, USA

Metabolic syndrome (MetS) is a spectrum of disorders including hypertension, dyslipidemia and obesity, all of which are major risk factors for cardiovascular disease, diabetes and premature death. Methionine sulfoxide reductase A (MsrA) regulates cell signaling and protects end-organs from oxidative damage. Polymorphisms in or near the MSRA gene locus are associated with visceral fat deposition, obesity, type 2 diabetes and hypertension in humans. We previously demonstrated that MsrA deficient (-/-) mice exhibit autonomic dysregulation and hypertension [Clin Auton Res 2012]. In this study we tested the hypothesis that decreased MsrA expression exacerbates metabolic dysfunction in mice fed a high-fat diet (HFD). Metabolic phenotypes were measured in young MsrA heterozygote $(+/-, n=8)$, homozygote $(-/-, n=7)$, and littermate wild-type (WT) control $(n=5)$ mice, before and after implementing the HFD (60\%). WT mice fed HFD for $\sim 10$ weeks exhibited increases in body weight, $\%$ body fat, fasting glucose, and serum cholesterol and triglyceride levels, and decreased glucose tolerance compared with baseline levels $(\mathrm{P}<0.05)$. The HFD-induced abnormalities in cholesterol, triglycerides, glucose and glucose tolerance were exacerbated in MsrA+/- (vs. WT) mice $(\mathrm{P}<0.05)$, despite measurements in the heterozygote mice being normal while on normal diet. The results demonstrate a significant MsrA gene-HFD interaction. MsrA-/- mice on HFD exhibited the highest body weights, \% body fat, and cholesterol and triglyceride levels; and the most impaired glucose tolerance. We conclude that decreased MsrA expression exacerbates metabolic dysfunction in mice fed a HFD. This discovery provides rationale for measuring MsrA expression in multiple end-organs affected in MetS, and development of drug- and gene-based therapies to restore MsrA expression.

Funding: NIH HL14388, AHA 17GRNT33670004.

\section{Poster \#73}

Angiotensin II type 2 receptors restore autonomic function and delay onset of dilated cardiomyopathy in male mice with muscular dystrophy

\section{Yang, K. Zimmerman, R.M. Weiss, R. Sabharwal}

Department of Internal Medicine, University of Iowa, Iowa City, IA, USA

Muscular dystrophies are heterogeneous genetic disorders that cause muscle atrophy and dilated cardiomyopathy (DCM). We have demonstrated that angiotensin II (Ang II) via type 1 receptors (AT1R) mediate autonomic dysfunction at young age (45 weeks) in the sarcoglycan delta deficient (Sgcd-/-) mice, an established model of muscular dystrophy [Exp Physiol 2013, 2015]. In many pathological conditions, activation of AT2R has been shown to offset deleterious actions of AngII/AT1R. We hypothesized that agonism of AT2R by a non-peptide small molecule compound 21 (C21; Vicore Pharma) ameliorates AngII-induced autonomic dysfunction and delays development of DCM in Sgcd-/- mice. Control C57BL6 ( $n=5-8)$ and Sgcd-/- $(\mathrm{n}=6-10)$ male mice were infused with vehicle (saline) or $\mathrm{C} 21$ ( $0.03 \mathrm{mg} / \mathrm{kg} / \mathrm{min}$, minipump) for eight-ten weeks, beginning at 3 weeks of age for group 1 (younger), and at 45 weeks of age for group 2 (older) experiments. Mean blood pressure (BP), heart rate (HR) and locomotor activity were recorded by telemetry, while cardiac function was assessed by echocardiography. Autonomic indices including baroreflex sensitivity (BRS, sequence technique) and resting cardiac vagal and sympathetic tone (HR responses to atropine and propranolol) were calculated. Group 1: Sgcd-/- mice exhibit lower BP, reduced $\mathrm{BRS}$ and vagal tone, and increased sympathetic tone $(\mathrm{P}<0.05$ vs. control). C21 improved BRS and restored sympathovagal balance in Sgcd-/- mice ( $\mathrm{P}<0.05$ vs. vehicle). Importantly, $\mathrm{C} 21$ normalized activity and AT1R expression in Sgcd-/- mice. Group 2: Older-aged Sgcd-/- mice exhibit increased left ventricular end-diastolic and endsystolic volumes, and reduced ejection fraction. C21 markedly improved cardiac function in Sgcd-/- mice, and persisted for an additional two weeks after C21 treatment was discontinued in Sgcd-/mice. Thus, we conclude that activation of AT2R is a potential novel treatment strategy that alleviates muscular dystrophy phenotype and delays the onset/severity of dilated cardiomyopathy.

Funding: AHA 17GRNT33670004. 


\section{Poster \#74}

\section{Effect of the menstrual cycle on resting, exercise and post-exercise} heart rate in healthy women

C. Giovanna, G. Porto Luiz Guilherme, M. Daniel, S. Edgard,

G. Giliard, C. Carlos Janssen, E. M. Guilherme

University of Brasilia, Brazil

Introduction: Spontaneous heart rate (HR) is an important physiological measurement in the clinical and exercise contexts, because HR is normally used as a parameter to diagnose, control and prescribe exercise. However, it is not totally clear whether spontaneous HR could be influenced by menstrual cycle.

Purpose: Our objective was to investigate the effect of menstrual cycle (follicular and luteal phases) on the HR at rest, during the maximal treadmill exercise (MTE) and post-exercise recovery in a clinically healthy woman.

Methods: We evaluated 11 healthy women $(24.1 \pm 4.4$ years, BMI $22.5 \pm 0.6 \mathrm{~kg} / \mathrm{m}^{2}$ ) with menstrual cycle lasting $28 \pm 1.1$ days. At rest, the HR was recorded in the supine and orthostatic positions. During the MTE, we recorded the chronotropic reserve (HRmax - HRIinitial) and in the post-exercise recovery we recorded the HR recovery (HRR) at the 1st, 3th, and 5th min following MTE. The evaluations were performed in the follicular phase between the 9th and 11th day and in the luteal phase between the 19th and 21st day after the beginning of the menstrual cycle. Statistical analysis employed parametric tests with two-tailed $\mathrm{p}$ value set at $5 \%$.

Results: At rest, HR was [supine: $64 \pm 2.8 \mathrm{bpm}-64.7 \pm 1.9 \mathrm{bpm}$, ( $\mathrm{p}>0.05)$ ] and [orthostatic: $82.6 \pm 3.4-82.1 \pm 3.4 \mathrm{bpm}(\mathrm{p}>0.05)$ ] during the follicular and luteal phases, respectively. Chronotropic reserve was not different ( $\mathrm{p}>0.05)$ during follicular $(86.4 \pm 2.2 \mathrm{bpm})$ and luteal $(86.9 \pm 3.8 \mathrm{bpm})$ phases. Also, absolute HRR, $\Delta \%$ HRR and HRR Coefficient did not show any difference between both phases of menstrual cycle $(\mathrm{p}>0.05)$.

Conclusion: We concluded that amendments of the menstrual cycle in female physiology, throughout the follicular and luteal phases do not change $(\mathrm{p}<0.05)$ the HR responses during resting conditions, maximal treadmill exercise and post-exercise recovery in healthy women. HR responses during rest, maximal treadmill exercise and 5 minutes postexercise were not affected by the menstrual cycles, even with all physiological changes that occur during follicular and luteal phases.

\section{Poster \#75}

\section{Sympathetic neural traffic in older patients with type 2 diabetes mellitus}

K. Heusser, J. Tank, B. Johannes, J. Jordan

Institute of Aerospace Medicine, German Aerospace Center, Cologne, Germany

Introduction: Mechanistic studies suggested that excess sympathetic activity promotes arterial hypertension while worsening insulin sensitivity. The finding raised interest in interventions targeting the sympathetic nervous system. Older patients with type 2 diabetes are at particularly high cardiovascular and metabolic risk. However, data on sympathetic activity in this population is scarce.

Material and Methods: We recruited patients with type 2 diabetes mellitus for at least 2 years, an age of 50-80 years, an HbA1c of 6.5$10.0 \%$, and a BMI of $25-40 \mathrm{~kg} / \mathrm{m}^{2}$. They had to be treated with stable doses of metformin only. In the supine position, we recorded ECG, brachial blood pressure, and muscle sympathetic nerve activity
(MSNA). We quantified MSNA as burst frequency, burst incidence, and burst area.

Results: We included 37 patients (14 women, $63 \pm 1$ years, $32.8 \pm 0.8$ $\mathrm{kg} / \mathrm{m}^{2} ; 23$ men, $64 \pm 1$ years, $30.3 \pm 0.7 \mathrm{~kg} / \mathrm{m}^{2}$ ). Blood pressure was $127 \pm 4 / 74 \pm 3 \mathrm{mmHg}$ in women and $126 \pm 3 / 77 \pm 2 \mathrm{mmHg}$ in men $(\mathrm{p}=0.92 / 0.42)$. Heart rate was $68 \pm 3 \mathrm{bpm}$ in women and $62 \pm 1 \mathrm{bpm}$ in men $(\mathrm{p}=0.03)$. Overall, MSNA ranged between 15 and 72 bursts $/ \mathrm{min}$. MSNA was $39.5 \pm 3.5$ bursts/min in women and $38.5 \pm 2.3 \mathrm{bpm}$ in men $(\mathrm{p}=0.80)$. Similarly, MSNA burst incidence and burst area did not differ between women and men. In the pooled analysis, MSNA was not correlated with systolic blood pressure, diastolic blood pressure, body mass index, or HbA1c (r2 0.30 for all).

Conclusions: In older patients with type 2 diabetes mellitus, MSNA appears to be not or only weakly related to gender, blood pressure, body mass index, or glycemic control. The findings suggest that sympathetic contributions to blood pressure and glucose metabolism are highly variable in this population, which may have implications for therapeutic interventions targeting the sympathetic nervous system.

\section{Poster \#76}

\section{Autonomic medical practice as sampled by physician-rating} websites

W.P. Cheshire

Department of Neurology, Mayo Clinic, Jacksonville, FL, USA

Objective: To survey online patient ratings of physicians practicing autonomic medicine.

Background: $70 \%$ of patients as healthcare consumers look to online reviews when searching for a physician. Website reviews score physicians on 5-point scales based on self-selected patients' perceptions of physician's knowledge, diagnostic accuracy, promptness, waiting time, staff friendliness, and follow up. The total score emphasizes patients' subjective experience.

Design/Methods: The study group consisted of all members of the American Autonomic Society (AAS) who are physicians practicing in the US who have been rated by the three most commonly used physician-rating websites: Vitals.com, Healthgrades.com, and RateMDs.com. A composite patient satisfaction score was calculated from total site scores divided by number of reviews. Scores were compared to indices of autonomic expertise: years in medical practice, number of publications in PubMed, and election by peers to AAS office. Comparisons were assessed by ANOVA and linear regression with significance defined as $\mathrm{P}<0.05$.

Results: The AAS directory lists 70 physicians practicing in the US, 64 ( 15 female, 49 male) of whom have been rated. Reviews numbered $24.0 \pm 32.9$ (mean \pm SD) per physician. Mean composite patient satisfaction scores were $3.80 \pm 0.84$. Scores correlated inversely with the number of scientific publications $(\mathrm{r} 2=0.05, \mathrm{P}=0.0065)$. Scores of AAS presidents were lower than those of other physician members (3.28 \pm 0.89 vs. $3.91 \pm 0.80, \mathrm{P}=0.023)$. Scores did not correlate with physician gender, number of reviews, or years in practice.

Conclusion: A quality patient experience, accurate diagnosis, and appropriate treatment are goals shared by patients and autonomic specialists. Whereas online reviews are sometimes useful, their validity is unproven, and their methodology relies on small sample sizes subject to selection bias. Of ethical concern is the potential for negative reviews to incentivize compromising evidence-based approaches to diagnosis and treatment in order to safeguard one's online professional reputation. 


\section{Poster \#77}

Fast heart rate recovery and its association with vagal withdraw after active orthostatic test in men

G.E. Molina, C.J.G. Cruz, M.T. Morlin, E.M.K.V.K. Soares, G.L. Garcia, L.G.G. Porto

Exercise Physiology Laboratory, Faculty of Physical Education, University of Brasília, Brazil

Introduction: The relationship between fast heart rate recovery (HRR) and the cardiovagal modulation responsiveness to active postural change at rest is still an open field to be explored.

Objective: To correlate fast HRR with cardiovagal status after an orthostatic maneuver (active stand from supine position) at rest. Method: HRR at the 1st, 2nd and 3rd min following sub-maximal treadmill exercise (reach a target heart rate within $85 \%$ of the agepredicted maximum) were correlated with relative change of timedomain index rMSSD - (parasympathetic index) after orthostatic maneuver of heart rate variability (HRV) in 18 healthy men. Statistical analysis employed non-parametric tests with two-tailed p-value set at $5 \%$.

Results: Coefficient of HRR (CHRR) at the 2nd and 3th min showed positive correlation with $\mathrm{rMSSD}$ ( $\mathrm{rs}=0.64-0.72, \mathrm{p}=0.01-0.02$ ). No correlation was found with rMSSD and HRR ( $\mathrm{rs}=0.28, \mathrm{p}=0.14$ ). CHHR was higher at the 2nd and 3rd min of post-exercise recovery after we used the 1st quartile of rMSSD responsiveness as cutoff point (Low: $\leq 1$ st quartile and high: $>1$ st quartile, responsiveness) The results were for CHHR at 2nd min - Low 51 (42-55)\% and High 64 $(57-68) \% ; \mathrm{p}=0.02$ and for CHHR at 3rd min - Low 54 (48-61)\% and High $70(62-74) \%$; $\mathrm{p}=0.02$.

Conclusion: Fast HRR during the initial 1st to 3rd minutes of the recovery period after sub-maximal treadmill exercise is positively correlated with the parasympathetic decrease (reserve) cardiovagal modulation after active postural change at rest in healthy men.

Funding: Fundação de Amparo a Pesquisa do Distrito Federal - DF.

\section{Poster \#78}

Acute effects of propranolol and pindolol on heart rate, metabolic rate, and cold-induced thermogenesis in lean, young men

R.J. Brychta ${ }^{1}$, R. Muniyappa ${ }^{1}$, S. McGehee ${ }^{1}$, S. Huang ${ }^{1}$, J.D. Hattenbach ${ }^{1}$, S.L. Bell ${ }^{1}$, A.B. Courville ${ }^{2}$, S. Yang ${ }^{2}$, B.P. Leitner ${ }^{1}$, C.J. Duckworth ${ }^{1}$, T. Cassimatis ${ }^{1}$, L.A. Fletcher ${ }^{1}$, H. Sasai ${ }^{1}$, A.M. Cypess ${ }^{1}$, M.L. Reitman ${ }^{1}$, K.Y. Chen ${ }^{1}$

${ }^{1}$ Diabetes, Endocrinology, and Obesity Branch, NIDDK, NIH, Bethesda, MD, USA; ${ }^{2}$ Nutrition Department, Hatfield Clinical Research Center, NIH, Bethesda, MD, USA

Background: Cold-induced thermogenesis, the increase in metabolic rate above basal during cold exposure, may be regulated through $\beta$ adrenergic receptors.

Aim: We tested whether acute doses of pindolol (a $\beta$-blocker with partial agonist, intrinsic sympathomimetic activity) and propranolol (without intrinsic sympathomimetic activity) had different effects on heart rate and metabolic rate in warm and cold conditions in healthy, lean, young men.

Methods: In a metabolic chamber, we measured resting metabolic rate and heart rate in 16 lightly clothed, fasted men (body mass index = $21.9 \pm 1.8 \mathrm{~kg} / \mathrm{m}^{2}$; age $26.1 \pm 4.5$ years) post-administration of placebo, $20 \mathrm{mg}$ pindolol, or $160 \mathrm{mg}$ propranolol during four hours of constant exposure to either a warm temperature $(27.0 \pm 0.7 \mathrm{C})$ or their coldest tolerated temperature before overt shivering $(21.3 \pm 1.4 \mathrm{C})$.
Results: In warm conditions, metabolic rate after placebo was $1.15 \pm 0.12 \mathrm{kcal} / \mathrm{min}$ and was not changed by pindolol $(1.12 \pm 0.13$ $\mathrm{kcal} / \mathrm{min})$ but was reduced by propranolol $(1.05 \pm 0.12 \mathrm{kcal} / \mathrm{min}$, $\mathrm{p}<0.01)$. The warm heart rate was $69.8 \pm 4.7$ beats $/ \mathrm{min}$, which was reduced by pindolol $(64.0 \pm 5.0$ beats/min, $\mathrm{p}<0.01)$ and more so by propranolol ( $55.9 \pm 4.7$ beats $/ \mathrm{min}, \mathrm{p}<0.01$ vs. pindolol). Cold exposure increased metabolic rate $(1.28 \pm 0.16 \mathrm{kcal} / \mathrm{min}, \mathrm{p}<0.01)$ and this was reduced by pindolol $(1.16 \pm 0.12 \mathrm{kcal} / \mathrm{min}, \mathrm{p}<0.01)$ and propranolol $(1.13 \pm 0.11 \mathrm{kcal} / \mathrm{min}, \mathrm{p}<0.01)$. Cold exposure reduced heart rate $(62.9 \pm 1.2$ beats $/ \mathrm{min}, \mathrm{p}<0.01)$, and this was further reduced by pindolol $(57.8 \pm 5.6$ beats/min, $\mathrm{p}<0.01)$ and propranolol $(54.6 \pm 5.6$ beats/ $\min , \mathrm{p}<0.01)$.

Conclusions: Propranolol reduced heart rate to similarly low levels in warm and cold, consistent with complete cardiac $\beta 1$-adrenergic receptor blockade. In contrast, propranolol reduced cold metabolic rate, but this remained above propranolol-inhibited warm metabolic rate. The effects of pindolol were generally intermediate between placebo and propranolol. These data indicate that pindolol's intrinsic sympathomimetic activity partially restores sympathetic components of heart rate and metabolic rate in warm and cold.

Funding: This work was supported by Intramural Research Program of the National Institute of Diabetes and Digestive and Kidney Diseases Grants Z01 DK071014 (K.Y.C.) and DK075116 (A.M.C.). S.H. was supported by Fudan University, Shanghai China and a fellowship from the China Scholarship Council during her doctoral work.

\section{Poster \#79}

Analysis of the speed of post-exercise heart rate recovery in athletes who practice triathlon and crossfit

\section{M.T. Morlin, C.J.G. Cruz, E.M.K.V.K. Soares, L.G.G. Porto,}

G.E. Molina

Exercise Physiology Laboratory, University of Brasília - UnB, Brazil

Recent studies have shown that sport modality can influence the resting heart rate (RHR), however, it is not clear whether sport modality can affect the speed of heart rate recovery (HRR). The aim of this study was to compare HRR in athletes who practice triathlon and crossfit after maximal exercise test. Cross-sectional study evaluated HRR at 1st, 3rd and 5th minutes of active recovery after a maximal cardiopulmonary test in a group of well-trained men $(n=30)$. The subjects were divided into 3 groups: (I) physically active (CG; $\mathrm{n}=10 ; 31 \pm 4$ years and BMI $23 \pm 3 \mathrm{~kg} / \mathrm{m}^{2}$ ); (II) an endurance triathlete group (TG; $\mathrm{n}=10 ; 32 \pm 4$ years and BMI $23 \pm 2 \mathrm{~kg} / \mathrm{m}^{2}$ ); and (III) Crossfit athletes group (CfG; $\mathrm{n}=10 ; 30 \pm 4$ years and BMI $26 \pm 2 \mathrm{~kg} /$ $\mathrm{m}^{2}$ ). Due to non-normality data assumptions, the Kruskal-Wallis test with post hoc Dunn was used to compare athletes HRR. No difference between groups were observed for age, maximal $\mathrm{HR}, \mathrm{VO}_{2}$ max and at 1 st minute of HHR ( $>0.05)$. However, absolute and relative differences were observed between TG (Abs 74 (70-87) bpm; \%=40 (3846)) vs. CfG (Abs $60(51-67) \mathrm{bpm} ; \%=32(27-37))(\mathrm{P}<0.01)$ at $3 \mathrm{rd}$ min of recovery, and only relative difference was observed between CG (Abs 63 (56-74) bpm; \%=32(29-40)) vs. CfG (Abs 74 (70-87) $\mathrm{bpm} ; \%=40(38-46)) \quad(\mathrm{P}<0.01)$. At 5 th $\min$ of recovery, where observed differences between CG (Abs 69 (62-77) bpm; \%=36 (3342) vs. TG (Abs 79 (74-91) bpm; \%=42 (41-48) $(\mathrm{P}<0.01)$ and between TG (Abs 79 (74-91) bpm; \%=42 (41-48) vs. CfG (Abs 69 (64-74) bpm; \%=37 (34-41)) $(\mathrm{P}<0.01)$. Therefore, the present results suggest that the sport modality practiced can influence the speed of HRR as well as RHR. 


\section{Poster \#80}

Neurotensin-induced relaxation of the guinea pig ileal circular muscle layer is indirect and mediated by release of vasoactive intestinal peptide

\section{H. Wang, D.M. Kendig, K.S. Murthy, J.R. Grider}

Department of Physiology \& Biophysics, Virginia Commonwealth University, Richmond, VA, USA; Department of Biology, Loyola University Maryland, Baltimore, MD, USA

Introduction: Neurotensin is a 13 amino acid brain-gut neuropeptide located in enteroendocrine cells and enteric neurons of the gastrointestinal tract. Recently we have shown that neurotensin acts in part to initiate the peristaltic reflex, which requires coordinated contraction and relaxation of gut muscle. The effects of neurotensin itself on smooth muscle preparations are variable depending on both the gut region and species, with both excitatory and inhibitory effects reported. The aim of this study was to determine the effect of neurotensin on the circular muscle of guinea pig ileum and release of the inhibitory neurotransmitter vasoactive intestinal peptide (VIP) from enteric neurons.

Methods: The effect of neurotensin (1-100 nM) was examined in isolated circular smooth muscle cells to determine contractile effects and in isolated muscle cells precontracted with $1 \mathrm{nM}$ cholecystokinin (CCK) to determine relaxant effects. Studies were repeated in innervated muscle strips from circular muscle layer and the medium collected for measurement of VIP by radioimmunoassay. To identify the role of NT1 receptors, experiments were repeated in the presence of the NT1 receptor antagonist SR48692 $(10 \mu \mathrm{M})$.

Results: In isolated smooth muscle cells, neurotensin alone had no effect on cell length and had no effect on cells precontracted with CCK. In innervated muscle strips, neurotensin alone had no effect but in strips precontracted with $\mathrm{CCK}$, neurotensin caused a concentrationdependent relaxation $(66 \pm 15 \%$ maximum relaxation at $100 \mathrm{nM}$; $E D 50=5 \mathrm{nM})$. Neurotensin-induced relaxation was accompanied by concentration-dependent VIP release which was maximal at $50 \mathrm{nM}$ (6.1 \pm 1.4 -fold increase above basal levels of $4.2 \mathrm{pg} / \mathrm{mg}$-min). Both relaxation and VIP release were strongly inhibited by the NT1 receptor antagonist.

Conclusion: Neurotensin causes an indirect relaxation of circular smooth muscle of guinea pig ileum by inducing release of the relaxant neurotransmitter VIP. This effect may enhance the descending relaxation phase of peristalsis leading to increased aboral propulsion. Funding: National Institute of Diabetes, Digestive and Kidney Diseases, DK34153.

\section{Poster \#81}

Consistent first trimester elevations in muscle sympathetic nerve activity and sympathetic baroreflex sensitivity between first and second pregnancy: a case study

C.E. Taylor ${ }^{1,2}$, S. Kobuch ${ }^{2}$, S.L. Hissen ${ }^{1}$, D. Boulton ${ }^{2,3}$, A. Burton ${ }^{2,3}$, N. Kramadhari ${ }^{2}$, S. Dayaratne ${ }^{2}$, V.G. Macefield ${ }^{2,3,4}$

${ }^{1}$ School of Science and Health, Western Sydney University, Sydney, Australia; ${ }^{2}$ School of Medicine, Western Sydney University, Sydney, Australia; ${ }^{3}$ Neuroscience Research Australia, Sydney, Australia,

${ }^{4}$ Baker Heart and Diabetes Institute, Melbourne, Australia

Research indicates that muscle sympathetic nerve activity (MSNA) is elevated during the first trimester of pregnancy. In a previous case study, we made measurements of MSNA during a normotensive pregnancy, reporting a peak in the first trimester. Here, we follow the same individual during their second pregnancy to assess the consistency of changes in MSNA and baroreflex sensitivity (BRS). Continuous measurements of blood pressure, heart rate and MSNA (microneurography) were recorded during $10 \mathrm{~min}$ of rest pre-pregnancy, during each trimester (weeks $6,11,16,22,28,32,37$ ) and post-partum (10 and 24 weeks following the birth). Bursts of MSNA were detected using Ensemble R and reported as MSNA burst incidence (bursts/100 hb). Sympathetic BRS was quantified as the relationship between diastolic pressure and MSNA burst incidence, and cardiac BRS using the sequence method. We report an identical peak in resting MSNA ( $\Delta 22$ bursts/100 hb) at 6 weeks for the two pregnancies. This was associated with upward resetting of the baroreflex and increases in sympathetic BRS from pre-pregnancy for both the first (9.9 vs. 1.0 bursts $/ 100 \mathrm{hb} / \mathrm{mmHg}$ ) and second pregnancy ( 9.3 vs. 1.0 bursts $/ 100 \mathrm{hb} / \mathrm{mmHg}$ ). Blood pressure remained at normotensive levels throughout the gestational and post-partum periods. During both pregnancies resting heart rate increased steadily over the gestational period. This was mirrored by a decline in cardiac BRS which reached a nadir of $2.9 \mathrm{~ms} / \mathrm{mmHg}$ during the first pregnancy (pre-pregnancy $27.8 \mathrm{~ms} / \mathrm{mmHg}$ ) and $6.6 \mathrm{~ms} / \mathrm{mmHg}$ for the second pregnancy (pre-pregnancy $29.9 \mathrm{~ms} / \mathrm{mmHg}$ ). In both pregnancies, a return of each variable towards pre-pregnancy levels was evident post-partum. This case study suggests that the rise in MSNA early in the gestational period may be consistent in subsequent pregnancies and is associated with sympathetic baroreflex resetting and greater sympathetic BRS. However, despite normal resting blood pressures, consistent declines are apparent in the baroreflex control of heart rate.

\section{Poster \#82}

\section{Reproducibility of Valsalva maneuver derived baroreflex parameters}

C. Ustine, L. Conant, P. Simpson, G. Chelimsky, C. O'Hara, T. Chelimsky

Department of Neurology, Medical College of Wisconsin, Milwaukee, WI, USA

Background: The Valsalva maneuver (VM) can provide estimates of both vagal and sympathetic baroreflex sensitivity.

Objective: To update data on the intra-subject variability of VMderived baroreflex parameters.

Methods: Subjects in the IRB-approved ICECAN trial return 5 times in 6 months for a series of 4 valsalva maneuvers, using 15" and 40 mmHg supine and at 30 degrees until good recordings were obtained. As per Singer et al., the vagal baroreflex component (BRS_v) constitutes the RR interval response to a preceding change in BP, either during vagal excitation (BRS_vup, from Phase IV), or vagal inhibition (BRS_vdown, from Phase II early) while Pressure Recovery Time (PRT) to baseline during phase IV reflects adrenergic baroreflex. A custom MATLAB script selected the BP and RR interval correlation with the best R2 among 0,1 and 2 beat delays, and calculated BRS_v as the linear regression slope. Four subjects were presented in 2018. Improved methodology since 2018 included more precise time assessment for onset and offset of the pressure hold, and a longer baseline (15 vs. 5 seconds) prior to heart rate to assess baseline pressures prior to Valsalva onset. We examined $22 \mathrm{VMs}$ collected from 11 subjects over 2 visits and selected the highest quality VM for analysis based on the best R2 and absence of artifact. Due to the presence of outliers, Spearman correlations were used.

Results: Of the 22 Valsalva trials examined, R2 was $>0.84$ in all but 4. The median, 5th, 95th percentiles values were $0.0059,0.0019$, $0.0189 \mathrm{~ms} / \mathrm{mmHg}$ for BRS_vdown, $0.0059,0.002,0.0235 \mathrm{~ms} / \mathrm{mmHg}$ for BRS_vup and $2.93,1.19,4.15$ secs for PRT. Spearman 
correlations between the two visits were $0.645(\mathrm{p}=0.032)$ for BRSv_up and $0.382(\mathrm{p}=0.247)$ for BRS_vdown. The correlation between BRS_vup and BRS_vdown for visit 1 was $0.064(\mathrm{p}=0.853)$ and for visit 2 was $0.809(\mathrm{p}=0.003)$. The intra-subject correlation for PRT was 0.08 .

Discussion: The VM baroreflex parameters show some intra-subject reproducibility in this larger sample, including BRS_vup, BRS_vdown. Some of the findings are less robust compared to 2018 with more subjects in the analysis. It is curious that the best correlation was between BRS_vup and BRS_vdown for visit 2. Perhaps there is a learning effect of some type, with visit 1 values less consistent than visit 2 values due to subject comfort. There is little correlation between the BRS values which represent primarily vagal baroreflex function, and the PRT which reflects primarily adrenergic baroreflex function.

Funding: NIH Diabetes, Digestive and Kidney Diseases, R01DK083538.

\section{Poster \#83}

\section{A case of dysautonomia in post-craniectomy syndrome of the trephined}

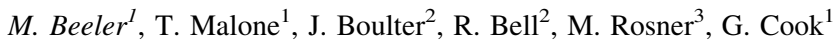
${ }^{1}$ Department of Neurology, Walter Reed National Military Medical Center, Bethesda, MD, USA; ${ }^{2}$ Department of Neurosurgery, Walter Reed National Military Medical Center, Bethesda, MD, USA;

${ }^{3}$ Department of Neurosurgery, George Washington University, Washington, DC, USA

Background: Cranial trephination, a surgical intervention resulting in a skull defect, has been performed for over 10,000 years to treat a wide variety of ailments and continues on today in the form of the craniectomy. Syndrome of the trephined (SoT) is a rare complication of craniectomies and symptoms include headache, focal neurologic deficits, seizures, and dysautonomia. These complications only resolve once a cranioplasty is performed. While SoT has been described in literature, little work has been done to quantify the dysautonomia these patients experience. We present a novel case of severe baroreflex dysfunction with orthostatic hypotension $(\mathrm{OH})$ related to craniectomy that resolved following cranioplasty.

Case Presentation: A 19-year-old man sustained a traumatic subdural hematoma $(\mathrm{SDH})$ resulting in cerebral herniation requiring an emergent decompression and SDH evacuation. He clinically improved over the next week, however he had anisocoria with a persistently dilated right pupil. MRI showed expected postoperative changes and T2 hyperintensity in the right cerebral peduncle but no brainstem pathology. Post-operatively, rehabilitation efforts were limited due to recurrent loss of consciousness with standing. $\mathrm{OH}$ was demonstrated on bedside testing, and formal autonomic demonstrated cardiovagal and sympathetic vasomotor reflex failure. Repeat autonomic testing 29 days after cranioplasty demonstrated recovery of his baroreflex function.

Results: Both cardiovagal and sympathoneural reflex failure suggests medullary dysfunction. Clinical evidence of injury to the peripheral right ocular motor nerve was present but no other neurologic findings consistent with cranial nerve or brainstem injury were. MRI demonstrated injury to the right cerebral peduncle, but no structural injury to the brainstem.
Conclusion: The mechanism by which SoT causes baroreflex failure is not understood. In this case we postulate increased atmospheric pressure resulted in sinking of the craniectomy skin flap and CSF displacement, along with increased craniocaudal flow of CSF, resulted in a transforaminal herniation leading to medullary dysfunction and dysautonomia.

\section{Poster \#84}

Assessment of practice patterns among neurologists and cardiologists for the care of patients with neurogenic orthostatic hypotension

T. Finnegan, J. Maeglin Medscape Education, New York, NY, USA

Introduction: Patients with neurodegenerative disorders often have autonomic dysfunction which places them at risk for neurogenic orthostatic hypotension $(\mathrm{NOH})$. Although a well-known consequence of neurodegenerative conditions, little is known about how NOH is diagnosed and managed in clinical practice. The goal of this study was to obtain a better understanding of the precise gaps in knowledge and competence of neurologists and cardiologists regarding the diagnosis and management of $\mathrm{NOH}$.

Methods: A continuing medical education (CME)-certified clinical practice assessment consisting of 25 multiple-choice questions on knowledge, clinical preferences, and competence was made available to neurologists and cardiologists in the United States. The questions were based on clinical trials, guidelines, and expert faculty recommendations regarding diagnosis and management of NOH. The survey posted on the Medscape Education website on March 20, 2019, with responses collected through May 2, 2019.

Results: 195 neurologists and 167 cardiologists completed the survey during the study period. $48 \%$ of cardiologists and $31 \%$ of neurologists were unable to correctly identify the appropriate differential diagnosis for a patient presenting with syncope. Less than $50 \%$ of clinicians in either group correctly identified the change in heart rate indicative of $\mathrm{NOH}$. Approximately one-quarter of clinicians correctly identified coat-hanger pain as a distinct presentation of $\mathrm{NOH}$. With regard to the management of $\mathrm{NOH}$, approximately $40-50 \%$ of clinicians in both groups were not aware that rasagaline could exacerbate $\mathrm{NOH}$ or the mechanism of action of midodrine. Lastly, approximately one-quarter of clinicians were able to correctly identify the appropriate dosing associated with the FDA-approved therapies for the treatment of $\mathrm{NOH}$.

Conclusions: This research yielded important insights into current clinical knowledge gaps of both neurologists and cardiologists regarding the diagnosis and management of $\mathrm{NOH}$. The results indicated a need for continued education on these topics as they relate to $\mathrm{NOH}$.

Funding: The educational programming and outcomes analysis was funded through an independent educational grant from Lundbeck. 


\section{Poster \#85}

\section{Prevention of vasovagal syncope using hyoscyamine}

\section{H. Snapper, M. Jawaid}

Department of Cardiology, WellStar Kennestone Regional Medical Center, Marietta, GA, USA

Introduction: Vasovagal syncope (VVS) is a very common cause of syncope [Grubb, N Engl J Med. 2005;352(10):1004-10]. As an isolated or rare event, therapy is usually unnecessary. Although benign, VVS can result in injury, significant functional impairment, and multiple hospital visits increasing the cost of healthcare [da Silva, Front Physiol. 2014;5:471]. Unfortunately, there are no known therapies associated with significant efficacy for prevention [Shen et al., J Am Coll Cardiol. 2017;70(5):e39-e110]. Considering the reflex paradoxical vagal response in VVS and the fact that anticholinergics have a relatively safe side effect profile, we investigated the efficacy of hyoscyamine in preventing VVS in a retrospective study design. Methods: We searched our electronic patient database using the terms "vasovagal syncope" and "hyoscyamine". Patients included in the study were those who had a diagnosis of VVS for which they were prescribed and using hyoscyamine and had documented follow-up. Results: The search resulted in 162 patients of which five were excluded due to use of hyoscyamine for reasons other than VVS. Of the 157 subjects included in the study, complete data set was available for 73 patients. 61 of the 73 patients $(83.6 \%)$ experienced improvement or resolution of their symptoms. 12 out of the 73 patients $(16.4 \%)$ did not find hyoscyamine useful or quit the medication due to an adverse event.

Conclusion: Hyoscyamine appears to be an effective medication for the prevention of VVS.

\section{Poster \#86}

Norepinephrine transporter inhibition for the prevention of tiltinduced vasovagal syncope: a systematic review and metaanalysis

L. Lei, A. Qaddoura, S.R. Raj, R.S. Sheldon MD

Libin Cardiovascular Institute, University of Calgary, Calgary, AB, Canada

Background: Vasovagal syncope is a common clinical problem that significantly reduces quality of life yet lacks effective medical therapies. Pharmacological norepinephrine transporter (NET) inhibition increases sympathetic tone, leading to suggestions that it might be able to prevent hypotension and syncope. The objective of this systematic review and meta-analysis was to evaluate the ability of selective NET inhibitors - reboxetine, sibutramine, and atomoxetine to prevent syncope in healthy subjects and patients with recurrent VVS.

Methods: We conducted a systematic search and meta-analysis of studies in MEDLINE, EMBASE, CENTRAL, and CINAHL without language restriction from database inception to March 2019. We included all randomized controlled trials that compared the effect of selective NET inhibitors versus placebo in healthy and VVS populations. Studies were required to report a dichotomous outcome of syncope or a predefined syncope surrogate.

Results: Four studies (101 subjects) met criteria for inclusion. The mean study size was 25 (range 11-56) participants. Three studies tested the ability of NET inhibition to prevent syncope induced by tilt table testing in healthy subjects (I $2=0 \%$ ). NET inhibition drastically reduced the likelihood of positive test outcomes (Relative Risk
$(\mathrm{RR})=0.14 ; 95 \%$ CI: $0.04-0.48 ; \mathrm{p}=0.002$ ). One study (the Prevention of Syncope Trial 6) examined the efficacy of NET inhibition in patients with recurrent vasovagal syncope and found a similar effect of atomoxetine in preventing syncope induced by tilt-table testing ( $\mathrm{RR}=0.49 ; 95 \%$ CI: 0.28-0.86; $\mathrm{p}=0.01)$.

Conclusion: NET inhibition is very effective at the prevention of syncope induced by tilt-table testing in both healthy participants and patients with recurrent vasovagal syncope. Pharmacological NET inhibition holds promise as a potential treatment for recurrent syncope given the 51\% relative risk reduction in vasovagal syncope patients. A formal randomized controlled clinical trial is necessary to properly assess the clinical relevance of NET inhibition as an effective treatment for vasovagal syncope.

\section{Poster \#87}

Does recent weight loss predispose to vasovagal syncope? a casecontrol series

\author{
B. Cumming, D. Jardine \\ Department of General Medicine, Christchurch Hospital, \\ Christchurch, New Zealand
}

Aim: Weight loss after bariatric surgery has been observed to cause orthostatic intolerance. Vasovagal syncope (VVS) following moderate weight loss outside of the setting of bariatric surgery is not well described. This study examines the association between onset of recurrent syncope and recent weight loss.

Methods: A retrospective clinical record review was undertaken in a syncope clinic between May 2015 and January 2018. Patients with VVS who had recent weight loss (WL) were included, and matched with age, gender and weight-matched controls without weight loss from the same clinic.

Results: $31 \mathrm{WL}$ patients (21 female and 10 male) and 31 controls were compared. The mean age was 46.5 years (range $18-81$ years) and mean weight $73.6 \mathrm{~kg}$. The mean WL was $22.7 \mathrm{~kg}$ (range $5-114 \mathrm{~kg}$ ). WL had occurred over a period of one year or less in $39 \%$ of patients. Only $16 \%$ had undergone bariatric surgery: other patients reported lifestyle modification (35\%), gastrointestinal disease (10\%), or unknown cause (32\%). In $77 \%$ of WL patients the vasovagal symptoms had been present for one year or less, compared with $65 \%$ of controls (OR 2, p=0.2). Only $23 \%$ of WL patients had a prior history of fainting, compared with $58 \%$ in the control group (OR 0.08 , $\mathrm{p}=0.001$ ). Haemodynamic recordings demonstrated very similar blood pressure levels in the two groups during the first five minutes of head-up tilt: mean supine blood pressure was $116 / 57 \mathrm{mmHg}$ in WL patients compared to $119 / 67$. During head-up tilt, only one WL patient and three controls had classical orthostatic hypotension.

Conclusion: Moderate recent weight loss is not currently listed as a predisposing factor for orthostatic intolerance or vasovagal syncope. This study demonstrates the association between the onset of recurrent vasovagal syncope and recent moderate weight loss. WL patients were significantly more likely to have no prior history of fainting, compared with controls. The most common finding during tilt table testing was low-normal supine blood pressure without postural hypotension. 


\section{Poster \#88}

Factors influencing patients' decision making around accessing emergency medical services for syncope

\section{T. Runte ${ }^{1}$, T. Williamson ${ }^{3}$, R. Sheldon ${ }^{3}$, T. Campbell ${ }^{3}$, K. King-} Shier $^{3}$, S.R. Raj ${ }^{3}$, M. Runte ${ }^{2}$

${ }^{1}$ Faculty of Science and ${ }^{2}$ Dhillon School of Business, University of Lethbridge, Lethbridge, AB, Canada; ${ }^{3}$ Department of Cardiac Sciences, University of Calgary, AB, Canada

Background: Syncope causes 1-3\% of emergency department (ED) admissions with $65 \%$ of syncope patients seen in ED arriving by ambulance. Little is known about the motivations prompting syncope patients to access emergency services (ES).

Objective: Exploratory qualitative research identified the factors prompting syncope patients to access ES. Results informed development of a paramedic-run ED "treat and refer" $(\mathrm{T}+\mathrm{R})$ for syncope patients, whereby they will receive assessment, education and followup facilitated by paramedic services.

Methods: A convenience snowball sample of self-identified "repeat fainters" (aged 18-67 years) were interviewed. Participants were asked to discuss their reasons for contacting or not contacting ES and their perception of the process in terms of meeting needs and expectations. Participants were then asked to discuss the potential for a $\mathrm{T}+\mathrm{R}$ program administered by paramedic services.

Results: Patients draw upon past experiences of engaging with emergency care as well as incident specific factors, such as the novelty of symptoms, injury from fall and the influence of bystanders, when making the decision to access or not access emergency care. Individual factors, such as patient age and co-morbidities, were also salient.

Funding: Canadian Arrhythmia Network of Canada (CANet) through the Networks of Centres of Excellence.

\section{Poster \#89}

Effect of a neck compression collar on cardiorespiratory function in postural tachycardia syndrome (POTS)

M. Nardone, J. Guzman, P. Harvey, J. Floras, H. Edgell School of Kinesiology, York University, Toronto, ON, Canada

The neck compression collar (Q-collar) was developed to reduce injury to the brain caused by head impacts in athletes by increasing brain blood volume. Neck compression can influence arterial baroreceptors and chemoreceptors, and postural tachycardia syndrome (POTS) patients are known to have impaired baroreflex sensitivity (Swift et al., 2005) and enhanced chemoreflex function (Taneja et al., 2011). Therefore, we tested the device in patients with POTS ( $\mathrm{n}=10$, one male; age: $36 \pm 10$; BMI: $24 \pm 4 \mathrm{~kg} / \mathrm{m}^{2}$ ) to determine if wearing the collar would influence autonomic function, cardiorespiratory responses, and brain blood flow during paced deep breathing (6 breaths/min), Valsalva (exhalation to $40 \mathrm{mmHg}$ for 15 s), and 5 minutes of $70^{\circ}$ upright tilt. Trials with or without the collar were randomized. Participants were equipped to measure ECG, beat to beat blood pressure, end-tidal gases, and transcranial Doppler. We found that wearing the collar 1) decreased the normalized low-frequency component of heart rate variability at rest (No collar: $60 \pm 18$ nu, Collar: $51 \pm 23 \mathrm{nu}, \mathrm{P}=0.044), 2$ ) increased the exhalation to inhalation ratio during paced deep breathing (No collar: $1.22 \pm 0.08$, Collar: $1.27 \pm 0.11, \mathrm{P}=0.040$ ), 3) decreased end-tidal $\mathrm{O}_{2}$ (No collar: Baseline: $115 \pm 5 \mathrm{mmHg}$, Tilt: $122 \pm 10 \mathrm{mmHg}$; Collar: Baseline: $112 \pm 5 \mathrm{mmHg}$, Tilt: $118 \pm 5 \mathrm{mmHg} ; \mathrm{P}=0.026)$ and respiratory rate
(No collar: Baseline: $13 \pm 3$ breaths/min, Tilt: $18 \pm 5$ breaths/min; Collar: Baseline: $12 \pm 4$ breaths/min, Tilt: $15 \pm 4$ breaths/min; $\mathrm{P}=0.049)$ throughout the tilt, and 4) decreased the Vanderbilt Orthostatic Symptom Score (No collar: 27 \pm 13 , Collar: 19 \pm 13 , $\mathrm{P}=0.035)$ due to reductions in blurred vision $(\mathrm{P}=0.037)$, tremulousness $(\mathrm{P}=0.053)$, chest discomfort $(\mathrm{P}=0.047)$, and lightheadedness $(\mathrm{P}=0.025)$. There were no effects of the collar on end-tidal $\mathrm{CO}_{2}$ or brain blood flow velocity $(\mathrm{P}>0.05)$. Our results indicate that wearing the Q-collar increased parasympathetic control of heart rate, decreased sympathetic control of heart rate, decreased ventilation, and decreased pre-syncopal symptoms in POTS. Future studies should investigate longer term use of the collar during performance of daily activities in POTS patients.

Funding: Q-collars provided by Q30 Innovations. Funding provided by the Faculty of Health, York University.

\section{Poster \#90}

T cell surface marker, CRTH2 expression in postural tachycardia syndrome

H.A. Abdallah, H.M. Abdallah, C. Kim, O. Alpan

Department of Cardiology, Children's Heart Institute, McLean, VA, USA

Background: The underlying etiology and pathophysiology of postural tachycardia syndrome (POTS) are still unknown but are likely to be heterogeneous. Mast cell activation disorder is frequently encountered as a comorbidity in POTS, however, major debate still exists as to whether it is an underlying etiology for POTS or is consequence of the associated sympathetic activation that is frequently encountered in POTS. Overall, patients with mast cell activation disorder are more symptomatic.

Methods: Flow cytometry was performed on 20 patients with POTS $(\mathrm{M}: \mathrm{F}=1: 4$, age 16-34 y) and hemodynamic parameters that were obtained during tilt table testing were then compared to $\mathrm{T}$ cell surface CRTH2, one of the biomarkers of mast cell activation.

Results: There was a significant correlation $(\mathrm{p}<0.005)$ between memory CD4T cell CRTH2 expression (CD3CD4ROCRTH2) and maximum heart rate incremental increase in HUT in patients with POTS.

Conclusions: These results show, for the first time, a correlation between a $\mathrm{T}$ cell surface marker, CRTH2, and a specific hemodynamic abnormality in patients with POTS. This finding raises the question on whether targeting CRTH2 would lead to better symptomatic control in patients with POTS and mast cell activation.

\section{Poster \#91}

\section{Sleep disturbances in postural tachycardia syndrome}

T. Fayyaz, A. Sheikh, S.B. Alam, M.Y. Bilal, H. Nazeer, M. Farooq, S. Shaheen, H. Mistry, S. Usmani, N. Chaudhary, W. Almardini, A. Suleman Department of Research, The Heartbeat Clinic, Dallas, TX, USA

Postural tachycardia syndrome (POTS) is a form of autonomic dysregulation associated with an excessive increase in heart rate upon standing. The purpose of this study was to examine the effect of POTS on various factors affecting sleep. Tilt table tests in the electronic medical records system at the Heartbeat Clinic in Dallas, Texas were retrospectively reviewed for patients with the diagnosis of POTS. Patients were then randomly selected and contacted via 
telephone for interviews conducted by using standard questionnaires. The assessment included hours of sleep, sleep quality on a scale of 1-10 (1=lowest quality, $10=$ highest quality), trouble falling asleep and staying asleep, number of times waking up, reason(s) for waking up, waking up refreshed or with headaches, using sleep aids and any clinical diagnosis affecting sleep. Of 485 patients randomly contacted we received 150 responses; out of which 140 were females and 10 were males. The average hours of sleep per night were $7.13( \pm 1.95)$, average sleep quality was 5.66 (on a scale of 1-10) with SD 1.99; average times of waking up at night was 3.25 , average times of waking up due to pain was 1.61 . As for sleep aids, $16.66 \%(25 / 150)$ used OTC and $20.0 \%(30 / 150)$ used prescription drugs. The percentage of patients who had trouble staying asleep was $63.3 \%$ (64/ $150)$, trouble falling asleep $60.0 \%(62 / 150)$, woke up with headaches $59.3 \%$ (89/150), snored $35.3 \%$ (53/150), woke up refreshed $24.0 \%$ (36/150), obstructive sleep apnea (OSA) diagnosis $16.0 \%(24 / 150)$. Reasons for waking up included: pain $36.0 \%$ (54/150), not sure $28.66 \%$ (43/150), racing heart/dysautonomia $22.67 \%$ (22/150), miscellaneous $19.99 \%$ (30/150) (including body aches, nightmares, acid reflux, etc.). Reasons for staying awake: pain $29.33 \%$ (44/150), racing mind $13.33 \%(20 / 150)$, stress $8.0 \%(12 / 150)$, anxiety $7.33 \%(11 / 150)$, racing heart/dysautonomia $12.67 \%$ (19/150), miscellaneous $28.66 \%$ (43/150) (including restlessness, noise, insomnia, etc.). As for diagnosis affecting sleep: POTS 57.33\% (55/150), EDS $41.33 \%(62 / 150)$, MALS $7.33 \%$ (11/150), MCAD 6.00\% (9/150), anxiety $7.33 \%(11 /$ 150), fibromyalgia $4.67 \%$ (7/150), miscellaneous $50.0 \%(75 / 150)$ (including depression, migraines, neuropathy, chiari malformation, narcolepsy, etc.). Given the high percentage (41.33\%) of patients reporting sleep disturbance from pain, it is highly possible that this is due to an underlying joint hypermobility which is a very common secondary cause of POTS, it can be inferred that the connective tissue disorder and resulting autonomic dysregulation are greatly affecting the sleep quantity and quality for these patients.

\section{Poster \#92}

\section{Acute modafinil and cognition in postural tachycardia syndrome}

A.C. Arnold ${ }^{1,3}$, K. Haman ${ }^{3}$, E.M. Garland ${ }^{3}$, A.J. Miller ${ }^{1}$, M. Wang ${ }^{2}$, B. Shen ${ }^{2}$, S.Y. Paranjape ${ }^{3}$, B.K. Black ${ }^{3}$, A. Diedrich ${ }^{3}$, D. Robertson ${ }^{3}$, C.A. Shibao ${ }^{3}$, I. Biaggioni ${ }^{3}$, S.R. Raj ${ }^{3,4}$

Departments of ${ }^{1}$ Neural and Behavioral Sciences and ${ }^{2}$ Public Health Sciences, Penn State College of Medicine, Hershey, PA, USA;

${ }^{3}$ Division of Clinical Pharmacology and Autonomic Dysfunction

Center, Nashville, TN, USA; ${ }^{4}$ Department of Cardiac Sciences, Libin Cardiovascular Institute of Alberta, University of Calgary, Calgary, Canada

Background: Cognitive dysfunction or "brain fog" is an almost universal symptom in postural tachycardia syndrome (POTS), posing significant limitations to educational and occupational endeavors. Despite high prevalence and clinical impact, there have been no studies examining treatment strategies to improve cognition in POTS. In this study, we tested the hypothesis that acute administration of the psychostimulant modafinil, the beta-blocker propranolol, or the combination, could improve cognition in POTS.

Methods: We performed a randomized, double blind, placebo-controlled crossover study in 19 adult POTS patients admitted to the Vanderbilt Autonomic Dysfunction Center (100\% female, $32 \pm 7$ years of age, $16 \pm 2$ years education). Patients were withdrawn from psychotropic drugs and medications affecting autonomic activity or blood pressure for $>5$ half-lives. Patients received oral placebo, modafinil $(200 \mathrm{mg})$, propranolol $(20 \mathrm{mg})$, and modafinil plus propranolol (200 mg and $20 \mathrm{mg}$, respectively) in random order on four consecutive days. Seated cognitive testing was performed at 2.5 hours post-medication using CogState computerized brief test battery. Patients completed daily self-assessment surveys for positive and negative affect, mood, fatigue, and anxiety with changes in these outcomes reported from before drug to after cognitive testing.

Results: Study drugs had no effects on cognitive measures including psychomotor function, attention, visual learning, working memory, and executive function. Compared with placebo, modafinil improved positive affect $(-3.5 \pm 6.6$ placebo vs. $0.7 \pm 4.8$ modafinil; $\mathrm{p}=0.029)$, and the combination of modafinil plus propranolol improved positive affect $(1.5 \pm 8.6 ; \mathrm{p}=0.014)$ and reduced depression symptoms $(0.7 \pm 1.2$ placebo vs. $0.1 \pm 0.4$ combination; $\mathrm{p}=0.010)$ and fatigue symptoms $(1.3 \pm 3.0$ placebo vs. $-0.4 \pm 2.9$ combination; $\mathrm{p}=0.013)$ in POTS.

Conclusions: These findings suggest that, in contrast to our hypothesis, acute modafinil does not improve cognition in POTS. The combination of modafinil with propranolol, however, induced moodelevating effects. Further studies are needed to examine the impact of chronic modafinil therapy, alone and in combination with propranolol, on cognition in POTS.

Funding: NIH U54 NS065736, NIH P01 HL56693, NIH UL1 TR000445.

\section{Poster \#93}

A new disease cluster: postural tachycardia syndrome, hypermobile Ehlers-Danlos syndrome/hypermobility spectrum disorder and mast cell activation syndrome

I. Cheung ${ }^{1}$, J. Holoff ${ }^{1}$, J. Guzman ${ }^{2}$, R.M. Londono ${ }^{3}, \mathrm{~S} . \mathrm{Walsh}^{4}$, P. Vadas ${ }^{1}$

${ }^{1}$ Division of Allergy and Clinical Immunology, St. Michael's Hospital, Toronto, ON, Canada; ${ }^{2}$ Syncope and Autonomic Disorders Unit, Department of Medicine, McMaster University, Hamilton General Hospital, Hamilton, ON, Canada; ${ }^{3}$ Division of Clinical and Metabolic Genetics, Department of Pediatrics, The Hospital for Sick Children and University of Toronto, Toronto, ON, Canada; ${ }^{4}$ Division of Dermatology, Sunnybrook Health Sciences Centre, Toronto, ON, Canada

Rationale: Patients with postural tachycardia syndrome (POTS) and hypermobility often describe symptoms suggestive of mast cell activation. Herein, we describe a novel phenotype, characterized by the linkage of hyper-adrenergic POTS, hypermobile type of EhlersDanlos syndrome/hypermobility spectrum disorder (hEDS/HSD) and mast cell activation syndrome (MCAS).

Methods: Patients were recruited from an allergy and immunology clinic at a tertiary care teaching hospital. Inclusion criteria included diagnoses of POTS and of hEDS/HSD. A diagnosis of MCAS was confirmed by the presence of validated symptoms of MCAS and elevated serum and/or urinary markers of mast cell activation.

Results: Thirty patients were identified who met diagnostic criteria for both POTS and hEDS/HSD. All 30 patients also fulfilled diagnostic criteria for MCAS. All 30 patients reported mild to marked improvements in MCAS symptoms in response to anti-mediator therapy.

Conclusions: Symptoms of a mast cell disorder are often reported by patients with both POTS and hEDS/HSD. In this cohort, these patients fulfilled consensus criteria for MCAS. Anti-mediator therapy was effective in ameliorating MCAS-associated symptoms in these patients. Neurologists and cardiologists should work together with allergy and immunology specialists in order to provide comprehensive care for patients with these three conditions. 


\section{Poster \#94}

\section{Prevalence of anxiety and depression in children with POTS: a retrospective study}

B. Kakavand, A. Centner, S. Centner, S. Hasan

Department of Cardiology, Child Behavior at Nemours Children's Hospital, Orlando, FL, USA

Introduction: Postural tachycardia syndrome (POTS) is a chronic form of orthostatic intolerance associated with a multitude of symptoms. There are conflicting reports on the incidence of depression and anxiety (D-A) in adult POTS. The information on children with POTS is scant. Presence of D-A is an important piece of information, as they can negatively affect patient's compliance with therapy. The prevalence of depression and anxiety among general adolescent population is $12.5 \%$ and $2.2 \%$, respectively.

Methods: We reviewed retrospectively data on pediatric patients with POTS, age 12-18 years, who underwent autonomic testing at Nemours Children's Hospital during May 2018-May 2019 and were screened for D-A. Severity Measure for Generalized Anxiety Disorder questionnaire was used for anxiety and PHQ-9 Modified for Adolescence (PHQ-A) for depression. The data were compared to historical data in the literature on incidence of D-A in children. The study was approved by the Nemours Institutional Review Board.

Results: 27 children with POTS and complete records were enrolled (female 26, age $15.8 \pm 1.6$ years). On average, POTS symptoms began $1.9 \pm 1.3$ years before diagnosis. Seven patients had no D-A. 20/27 (74\%) had moderate-severe anxiety, depression or both. 4/27 (14\%) had pure depression, 4/27 anxiety and 12/27 (44\%) had both conditions. One patient had mild symptoms. 11 patients took stable doses of psychotropic medications. After a follow-up period of 5.1 \pm 1.7 months of POTS therapy, 7 patients had follow-up questionnaires. In $4 / 7$ depression severity and in 3/7 patients anxiety improved. Patients were not actively treated for D-A, or their existing therapy was not altered during this time.

Conclusion: Anxiety and depression are very common among children with POTS. POTS therapy by itself may improve symptom burden of anxiety and depression.

\section{Poster \#95}

\section{Why do patients wear a compression garment for their POTS} symptoms (or not)?

\section{K.M. Bourne ${ }^{1}$, R.S. Sheldon ${ }^{1}$, S.R. Raj ${ }^{1}$, M. Runte ${ }^{2}$ \\ ${ }^{1}$ Cardiac Sciences, University of Calgary, Calgary, AB, Canada; ${ }^{2}$ Dhillon School of Business, University of Lethbridge, Lethbridge, $\mathrm{AB}$, Canada}

Background: Postural tachycardia syndrome (POTS) is a common form of orthostatic intolerance. Compression garments are frequently prescribed, and we sought to explore the patient experience with this non-pharmacological treatment.

Objective: To identify the benefits, downsides and decision-making process to wear a compression garment using exploratory qualitative research methods, in a sample of patients diagnosed with POTS.

Methods: Participants expressed interest in participating via the UCalgary Research website. Participants were Canadian residents and had a physician diagnosis of POTS. Patients participated in a short telephone interview, where they responded to questions regarding compression garments as a treatment in POTS. Data analysis included evaluation of social, emotional and financial variables influencing compression garment use.
Results: This study is still enrolling. Twelve female participants (age $38 \pm 2$ years) are included in this analysis. Nine participants were currently using compression garments to manage their POTS symptoms. All participants have tried compression garments. Participants had worn a variety of garment styles including socks, tights and shorts. Some participants reported positive benefits and others reported minimal to no benefits. Some participants wore the garment on a daily basis, and others had tried it a few times or only wore occasionally. Reduced limb swelling and fatigue, as well as improved orthostatic tolerance and concentration were reported. Limitations included the effort required to put the garment on and physical discomfort including the temperature and pressure of the garment on the abdomen and legs. Forecasted weather, the participant's schedule, symptoms, and clothing choices were factors that affected the decision to wear or not wear the garment. Patients consider garment appearance in day-to-day decisions about what garment to wear. Conclusion: Some patients may experience more benefits from compression garments than others. Discomfort from the garment and poor physical appearance of the garment may have negative implications in the decision to wear compression garments.

\section{Poster \#96}

\section{Autoimmunity and immunotherapy in postural tachycardia syndrome}

K. Sato ${ }^{1}$, A. Yamaga ${ }^{1}$, Y. Nishimura ${ }^{1}$, K. Shibata $^{1}$, T. Nakaoka ${ }^{1}$, Y. Sunami ${ }^{2}$, T. Hirai ${ }^{3}$, T. Nakane ${ }^{4}$, H. Sakura ${ }^{1}$

${ }^{1}$ Department of Medicine, Tokyo Women's Medical University Medical Center East, Tokyo, Japan; ${ }^{2}$ Department of Neurology, Tokyo Metropolitan Neurological Hospital, Tokyo, Japan; ${ }^{3}$ Department of Neurology, Teikyo University Hospital, Mizonokuchi, Kanagawa, Japan; ${ }^{4}$ Department of Neurology, Kumamoto University, Kumamoto, Japan

Introduction: Postural tachycardia syndrome (POTS), which is characterized by an exaggerated increase in heart rate while standing, is associated with symptoms of lightheadedness and fatigue. However, the pathophysiology of POTS is still unknown. Recently, the role of autoimmunity in POTS has been reported on in several studies. Also, the comorbidity of autoimmune diseases such as Sjogren's disease, systemic lupus erythematosus, and mast cell activation disease is well-known. Taken together, abnormal autoimmunity is one of the key features of POTS.

Methods: Eleven POTS patients ( $24 \pm 10$ years old, 8 females) were measured for auto-antibodies against the alpha-1 adrenergic receptor (A1-AR), alpha-2 AR (A2-AR), beta-1 adrenergic receptor (B1-AR), beta-2 adrenergic receptor (B2-AR), muscarinic cholinergic receptor 1 (M1), muscarinic cholinergic receptor 2 (M2), muscarinic cholinergic receptor 3 (M3), muscarinic cholinergic receptor 4 (M4), and muscarinic cholinergic receptor 5 (M5), as well as angiotensin II receptor type 1 (AT1R) auto-antibodies.

Results: Positive auto-antibodies of A1-AR, A2-AR were 7 and 3, respectively. However, there was no positive B1-AR, B2-AR, and M1 auto-antibody patients. Positive auto-antibodies of M2, M3, M4, and M5 were 2, 3, and 1, respectively. Two patients showed positive AT1R auto-antibodies. Two of the severely affected POTS patients (case 1: 30-year-old female, case 2: 14-year-old male) were also measured for ganglionic acetylcholine receptor ( $\mathrm{gAChR}$ ) auto-antibodies and treated by both intravenous immunoglobulin (IVIG) and steroid pulse therapy (sPLS). Case 1, of which gAChR auto-antibodies was positive, showed improved orthostatic symptoms and exercise time after the IVIG treatment. Case 2, of which A1-AR autoantibodies was positive but $\mathrm{gAChR}$ auto-antibodies was negative, did 
not have benefit from IVIG treatment but improved exercise tolerability after the sPLS treatment by a cardiopulmonary exercise test. Evaluating auto-antibodies among POTS patients might be beneficial for the treatment option.

\section{Poster \#97}

Efficacy of epidural blood patch as a treatment for chronic orthostatic intolerance: a case series

\section{Kinsella, M. Boedefeld, J. Stephens}

SSM Health, St. Clare Neuroscience Institute, St. Louis, MO, USA

Background: Chronic orthostatic intolerance (COI) and postural orthostatic tachycardia syndrome (POTS) are disabling conditions that are poorly understood and often resistant to treatment. Many patients with COI and POTS have postural headache suggesting intracranial hypotension, but prior investigations have not found evidence of spontaneous intracranial hypotension (SIH) and cerebrospinal fluid (CSF) leaks. We reviewed our experience with empirical epidural blood patching (EBP) following spinal imaging in this population.

Methods: Patients with suspected CSF hypovolemia underwent noncontrast MR myelography with MOBI views of entire spine, and MRI of the brain with contrast. A lumbar EBP was performed in select patients.

Results: In 33 patients (31 F, median age 37), 27 (82\%) had chronic orthostatic intolerance (COI), $10(30 \%)$ had evidence of POTS, 3 $(10 \%)$ has idiopathic sinus tachycardia. $25(76 \%)$ had postural headache. There were 13 (39\%) with joint hypermobility syndrome (Ehler Danlos syndrome, hypermobile variant), defined as Beighton score $>4 / 9$. There were $6(18 \%)$ with symptoms suggestive of mast cell activation syndrome (MCAS). Using non-contrast MR myelography and contrast-enhanced MRI of the brain, $25(76 \%)$ had dilated neural sheaths, diverticula, paraspinal fluid collections, dilated epidural veins, and features of sagging brain such as tonsillar migration. Several had prior Chiari malformation surgery. 17 (52\%) responded favorably to one or more epidural blood patches.

Conclusion: Epidural blood patching may provide substantial if temporary relief in patients with COI with or without POTS, postural headache, EDS, and MCAS.

\section{Poster \#98}

Family inheritance in postural tachycardia syndrome: insights from a cross-sectional community-based survey

L.E. Stiles $^{1,2}$, K. Bourne ${ }^{3}$, C.A. Shibao ${ }^{4}$, L.E. Okamoto ${ }^{4}$, E.M. Garland ${ }^{4}$, A. Gamboa ${ }^{4}$, A. Peltier ${ }^{4}$, A. Diedrich ${ }^{4}$, I. Biaggioni ${ }^{4}$, R.S. Sheldon ${ }^{3}$, S.R. Raj ${ }^{3,4}$

${ }^{1}$ Department of Neurology, Stony Brook University, Stony Brook, NY, USA; ${ }^{2}$ Dysautonomia International, East Moriches, NY, USA; ${ }^{3}$ Department of Cardiac Sciences, University of Calgary, Calgary, AB, Canada; ${ }^{4}$ Vanderbilt Autonomic Dysfunction Center, Vanderbilt University, Nashville, TN, USA

Background: Postural tachycardia syndrome (POTS) is a common form of orthostatic intolerance. This study sought to explore family inheritance patterns in POTS.

Methods: The "Diagnosis and Impact of POTS" is a structured online survey approved by the Vanderbilt University and University of Calgary IRB. Between July 2015 and January 2019, 7212 patients (or parents of minors) with physician diagnosed POTS completed the survey.

Results: Of all respondents, $11 \%$ report a relative diagnosed with POTS by a physician and $17 \%$ report a blood relative diagnosed with a form of orthostatic intolerance by a physician, including orthostatic hypotension $(34 \%)$, reflex fainting $(30 \%)$, and autonomic failure (4\%). Amongst 60 female identical twins, $28 \%$ reported both twins being diagnosed with POTS. While most respondents were female $(94 \%)$, males $(n=373)$ were significantly more likely to report a relative diagnosed with POTS $(14 \%$ vs. $11 \%, \mathrm{p}=0.031)$, most often a sister $(42 \%)$, mother $(25 \%)$ or aunt $(15 \%)$. Individuals diagnosed with Ehlers-Danlos syndrome (EDS) and POTS ( $\mathrm{n}=1504)$ were significantly more likely to report a relative diagnosed with POTS or orthostatic intolerance than POTS/non-EDS individuals (18\% vs. $8 \%$, $\mathrm{p}<0.001 ; 24 \%$ vs. $15 \%, \mathrm{p}<0.001$; respectively). There was a nonsignificant trend for individuals diagnosed with an autoimmune disease (AI) and POTS $(\mathrm{n}=956)$ to report a relative diagnosed with POTS ( $12 \%$ vs. $10 \%, \mathrm{p}=0.093)$. Individuals diagnosed with AI and POTS were significantly more likely to report a daughter or son diagnosed with POTS $(3.45 \%$ vs. $1.7 \%, \mathrm{p}<0.001 ; 2.2 \%$ vs. $1 \%, \mathrm{p}=0.003$, respectively).

Conclusions: A subset of individuals with POTS report one or more blood relatives with POTS. Identical twins, males, and individuals with Ehlers-Danlos syndrome or autoimmune disease are more likely to report POTS within their families. Future research should seek to identify potential genetic markers that may contribute to the development of POTS.

Funding: This work was supported in part by the National Heart, Lung, and Blood Institute Award Number P01 HL056693, by the National Center for Advancing Translational Sciences Award UL1 TR000445, the Canadian Institutes of Health Research grant MOP142426, and the Cardiac Arrhythmia Network of Canada grants SRG-15-P01-001 and SRG-17-P27-001.

\section{Poster \#99}

Somatic and autonomic findings in cancer-related base of the skull syndromes

A. Barboi, S. Pocica, S. Moffat, I. Kafkas, V. Patel

Department of Neurology, NorthShore University HealthSystem, Evanston, IL, USA

Introduction: Jugular foramen syndromes have been described extensively and attributed to various mechanisms. There is variable involvement of cranial nerves IX to XII and cervical sympathetic fibers. The literature is either focused on the somatic or autonomic manifestations. As part of our quality improvement initiative, we decided to look at both somatic and autonomic involvement in this syndrome.

Methods: We reviewed the clinical and autonomic testing data of the patients with baroreflex failure seen in our clinic between 2014 and 2019.

Results: We identified 23 patients, 5 women, average age 67. Most common cancer was squamous cell carcinoma: 7 tonsillar, 6 oropharynx, 4 tongue, 1 salivary gland, 3 Hodgkin's, and one each for breast and 4th ventricular ependymoma. All patients had radiation therapy and in addition 8 also had surgery and 9 had chemotherapy. The most common presenting symptom was syncope followed by orthostatic hypotension, paroxysmal hypertension, headache, vertigo, flushing. 5 patients had pupillary sympathetic fiber involvement. Autonomic reflex testing findings: all patients had reduced Valsalva ratios, and normal heart rate variability was noted in 7 patients. Most frequent pattern (12 patients) in patients with sympathetic 
involvement, was one of orthostatic hypotension flanked by supine hypertension. In 3 patients where the sympathetic function was normal, both supine and orthostatic hypertension were seen. In 3 patients with intact heart rate variability and abnormal sympathetic function, supine hypertension was absent.

Conclusion: Baroreflex failure is part of the jugular foramen syndromes after treatment for head and neck cancer, particularly if radiation therapy is employed. It is important to expand the classic clinical description as autonomic involvement has significant clinical implications.

\section{Poster \#100}

Efficacy and safety of droxidopa for the treatment of patients with symptomatic neurogenic orthostatic hypotension during 12 weeks of open-label treatment in a phase 4 study

S. Gorny ${ }^{1}$, L.A. Hewitt ${ }^{1}$, A. Lindsten ${ }^{2}$, S. Kymes ${ }^{3}$, A. Favit ${ }^{1}$

${ }^{1}$ US Medical, Lundbeck, Deerfield, IL, USA; ${ }^{2}$ Biostatistics, Lundbeck A/S, Copenhagen, Denmark; ${ }^{3}$ Health Economic and Outcomes Research, Lundbeck, Deerfield, IL, USA

Objective: To evaluate the long-term efficacy and tolerability of droxidopa in patients with symptomatic neurogenic orthostatic hypotension $(\mathrm{nOH})$ during open-label treatment in an ongoing phase 4 study (NCT02586623).

Background: Droxidopa is approved to treat $\mathrm{nOH}$ symptoms in patients with autonomic failure based on short-term clinical trial data. Greater information on the durability of clinical benefit is required.

Methods: Patients with symptomatic nOH received 12 weeks of openlabel treatment with an individually optimized droxidopa dose (100-600 mg, 3 times daily; previously identified in a $\leq 28$-day titration period). Efficacy outcomes included change from baseline in patient-reported assessments of $\mathrm{nOH}$ symptoms (Orthostatic Hypotension Symptom Assessment [OHSA]), impact of symptoms on daily activities (Orthostatic Hypotension Daily Activity Scale [OHDAS]), and clinician- and patient-rated Clinical Global Impression-Severity (CGI-S) scales. Supine blood pressure (BP) and adverse events (AEs) were recorded.

Results: After 12 weeks, patients $(\mathrm{N}=113)$ reported significant improvements from baseline in $\mathrm{nOH}$ symptoms (OHSA composite and 6 individual item scores changes; all $\mathrm{P}<0.0001)$. Treatment had a significant positive impact on daily activities (OHDAS composite and 4 individual item score changes) and on both clinician and patient CGI-S scores (all $\mathrm{P}<0.0001$ vs. baseline). Mean increases in supine systolic and diastolic BP at week 12 were 15.5 and $7.8 \mathrm{mmHg}$ from baseline measurements (117.9 and $71.4 \mathrm{mmHg}$, respectively; $\mathrm{P}<0.0001$ for both changes). AEs were reported by $64 \%$ of patients, most frequently falls (17\%), headache (13\%), and dizziness (9\%). An $\mathrm{AE}$ of supine hypertension was reported in $1(0.9 \%)$ patient.

Conclusion: During 12 weeks of open-label treatment, droxidopa was associated with significant improvement from baseline in $\mathrm{nOH}$ symptoms and activities of daily living. AEs were similar in nature and rate to other long-term studies. No clinically notable changes in supine hypertension were observed. These results are supportive of continued efficacy beyond 2 weeks of treatment with droxidopa. Funding: The data reported were derived from a research project funded by Lundbeck. All authors are employees of Lundbeck.

\section{Poster \#101}

A phase 2 dose-escalation study of ampreloxetine (TD-9855), a norepinephrine reuptake inhibitor, given once-daily to treat neurogenic orthostatic hypotension $(\mathrm{nOH})$ in subjects with synucleinopathies

H. Kaufmann ${ }^{1}$, I. Biaggioni ${ }^{2}$, A. Panneerselvam ${ }^{3}$, B. Haumann ${ }^{3}$, R. Vickery ${ }^{4}$

${ }^{1}$ Department of Neurology, New York University School of Medicine, New York, NY, USA; ${ }^{2}$ Department of Medicine, Vanderbilt University Medical Center, Nashville, TN, USA; ${ }^{3}$ Theravance Biopharma US, Inc., South San Francisco, CA, USA; ${ }^{4}$ Theravance Biopharma Ireland Limited, Dublin, Ireland

Objective: To assess the acute effects of ampreloxetine in subjects with symptomatic neurogenic orthostatic hypotension $(\mathrm{nOH})$.

Background: Failure of the autonomic nervous system results in $\mathrm{nOH}$ with inadequate increase in synaptic norepinephrine (NE) and maintenance of blood pressure (BP) following postural change to the upright position. Ampreloxetine is a novel NE reuptake inhibitor for the treatment of symptomatic nOH.

Methods: A multicenter, 2-part study of the acute effects of ampreloxetine versus placebo was conducted. Part A was an ascending single-dose, 5-day study with placebo on Day 1, followed by escalating doses of ampreloxetine $(2.5,5,10,20 \mathrm{mg})$, based on safety, tolerability, and pressor effect (change from baseline in seated systolic BP [SBP], duration of standing, $\mathrm{OH}$ symptoms). Part B was a one-day, double-blind, parallel-design study in which subjects were randomized to receive one dose of placebo or ampreloxetine (dose determined by Part A response; maximum, $15 \mathrm{mg}$ ).

Results: Of 34 enrolled subjects (mean age, 66 years), 29 (85\%) completed all dosing levels of Part A. Four hours after ampreloxetine $10 \mathrm{mg}$, mean (SD) increase from baseline in standing duration and seated SBP were 1.6 (3.6) minutes and 4.9 (20.1) $\mathrm{mmHg}$ more than 4 hours after placebo. The commonest adverse events (AEs) were headache and urinary tract infection; one subject discontinued treatment due to AE; no serious AEs were reported. In Part B, 5 subjects were randomized to ampreloxetine and 5 to placebo. Mean $(95 \% \mathrm{CI})$ difference in seated SBP between subjects receiving ampreloxetine and subjects receiving placebo was $29.9(7.6,52.3) \mathrm{mmHg}$.

Conclusion: In subjects with $\mathrm{nOH}$, single doses of ampreloxetine resulted in acute increase from baseline in seated SBP and standing duration 4 hours post-dose. Ampreloxetine was generally well-tolerated. These results support further assessment to evaluate longer-term effects of ampreloxetine on symptoms of nOH.

Funding: Theravance Biopharma. 